Marc Hieronimus (Hg.)

Historische Quellen im DaF-Unterricht

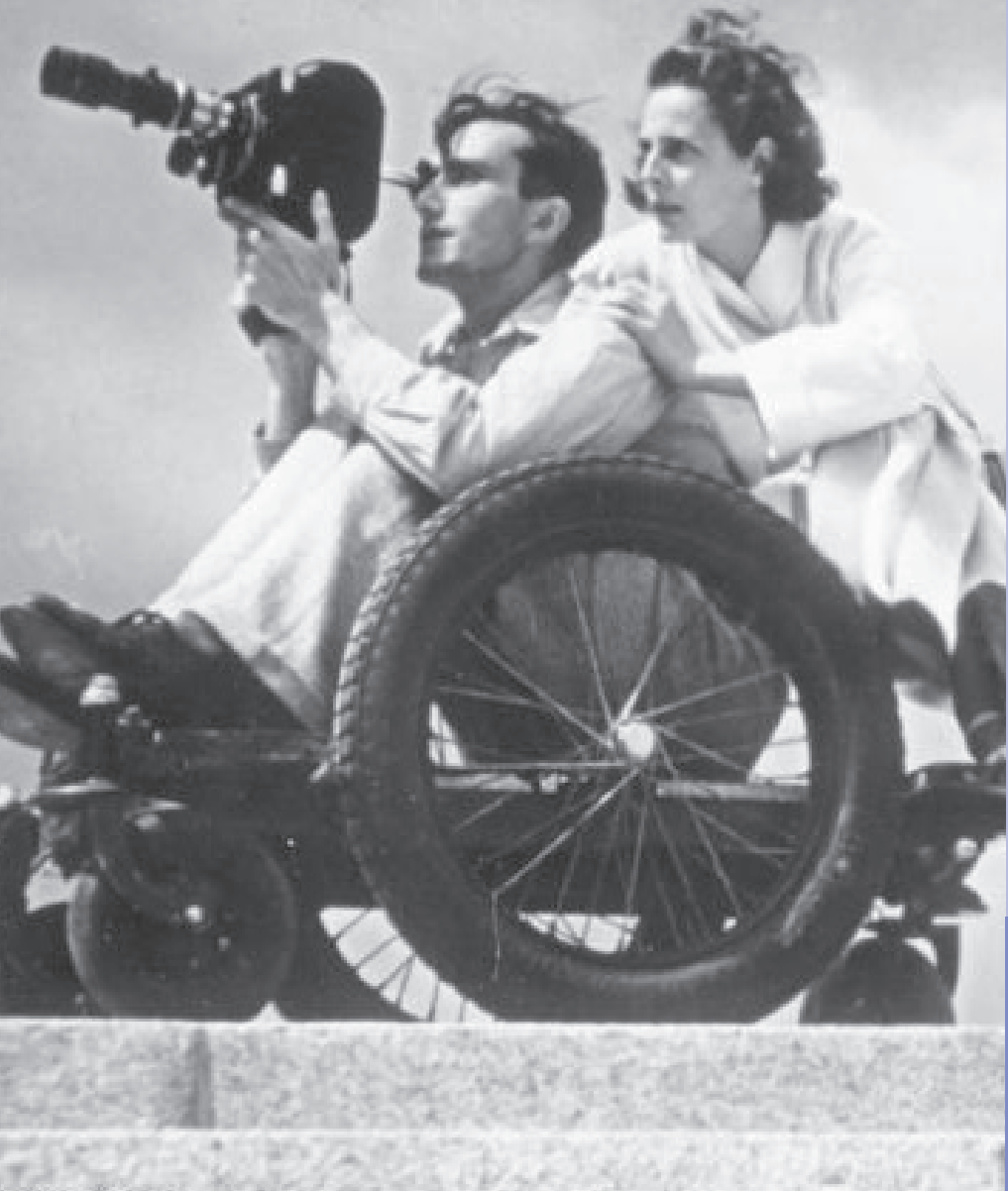

Band 86

Materialien

Deutsch als Fremdsprache

Universitätsdrucke Göttingen 

Marc Hieronimus (Hg.)

Historische Quellen im DaF-Unterricht

This work is licensed under the Creative Commons License 3.0 "by-nd", allowing you to download, distribute and print the document in a few copies for private or educational use, given that the document stays unchanged and the creator is mentioned.

You are not allowed to sell copies of the free version.

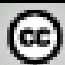

SORE RIGHIS RESERVED 
erschienen als Band 86 in der Reihe „Materialien Deutsch als Fremdsprache“ in den Universitätsdrucken im Universitätsverlag Göttingen 2012 
Marc Hieronimus (Hg.)

\section{Historische Quellen im DaF-Unterricht}

Materialien

Deutsch als Fremdsprache Band 86

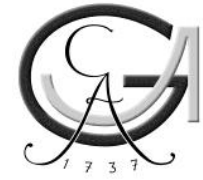

Universitätsverlag Göttingen 2012 


\section{Bibliographische Information der Deutschen Nationalbibliothek}

Die Deutsche Nationalbibliothek verzeichnet diese Publikation in der Deutschen Nationalbibliographie; detaillierte bibliographische Daten sind im Internet über $<$ http://dnb.ddb.de $>$ abrufbar.

Die „Materialien Deutsch als Fremdsprache“ sind eine Reihe des Fachverbands Deutsch als Fremdsprache e.V. $(\mathrm{FaDaF})$, in der Tagungsergebnisse, Dissertationen und andere wichtige Einzeldarstellungen aus dem Bereich Deutsch als Fremdsprache veröffentlicht werden.

http://www.fadaf.de/de/Publikationen/mat_daf/

\section{fadaf}

Schriftleitung: Annett Eichstaedt; Annegret Middeke

Dieses Buch ist nach einer Schutzfrist auch als freie Onlineversion über die Homepage des Verlags sowie über den OPAC der Niedersächsischen Staats- und Universitätsbibliothek (http://www.sub.uni-goettingen.de) erreichbar und darf gelesen, heruntergeladen sowie als Privatkopie ausgedruckt werden. Es gelten die Lizenzbestimmungen der Onlineversion. Es ist nicht gestattet, Kopien oder gedruckte Fassungen der freien Onlineversion zu veräußern.

Satz und Layout: Marc Hieronimus Umschlaggestaltung: Franziska Lorenz Titelabb.: Leni Riefenstahl bei Dreharbeiten (Bundesarchiv Bild 146-1988-106-29)

(C) 2012 Universitätsverlag Göttingen http://univerlag.uni-goettingen.de ISBN: 978-3-86395-061-3

ISSN: 1866-8283 


\section{Inhalt}

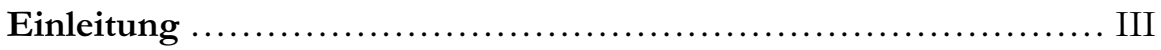

\section{Uwe Koreik}

Deutsche Geschichte im DaF-Unterricht -

zur Arbeit mit historischen Quellen ................................ 1

\section{Thomas Roth}

Historische Quellen im Internet.................................... 15

\section{Jürgen Nielsen-Sikora}

Fotografie als historische Quelle? ................................... 59

\section{Wolfgang Koller}

Der Film als historische Quelle

\section{Jens Grimstein}

Zeitgeschichtliche Filmszenen für den DaF-Unterricht -

Praktische Beispiele und Erfahrungen

\section{Beatrice Wiegand}

Historische Audio- und Videodokumente im DaF-/Phonetikunterricht -

Vorschläge und Erfahrungen aus der Unterrichtspraxis

\section{Jens Grimstein}

Formen der Arbeiterliteratur für den DaF- und Deutsch-Unterricht

\section{Arndt Kremer}

Namen schildern -

Straßennamen und andere Namensfelder im DaF-Unterricht ..

\section{Joachim Sistig}

Der Erste Weltkrieg in populärliterarischen Medien

\section{Marc Hieronimus}

Plakate im DaF-Unterricht

Autoren ...................................................... 237 



\title{
Einleitung
}

\author{
Marc Hieronimus
}

Die Kombination von Titel und Titelbild des vorliegenden Bandes mag manche Fachkraft für Deutsch als Fremdsprache stutzen machen: Das ist doch die Riefenstahl, beim Dreh eines dieser Propagandastreifen?! Da gibt man sich alle Mühe, den Lernern den Reichtum der Geschichte und Gegenwart der deutschsprachigen Länder aufzuzeigen und ihre hartnäckigsten Vor- und Fehlurteile zu korrigieren, hält sich also gar nicht allzu lange mit dem deutschen Trauma der NSZeit auf - und dann prangt da gleich auf dem Cover ein Foto aus just dieser Epoche!

Nun, zunächst handelt es sich bei dem Set-Foto um eine historische Quelle, sogar um eine Meta-Quelle, wenn der Ausdruck erlaubt sei: Wir sehen Leni Riefenstahl in jungen Jahren bei der Arbeit am Olympia-Film, der selbst Auskunft über Vergangenes erteilt. Die historisch vorbelastete Lehrkraft wird unschwer auch an diesem Foto von der Riefenstahl ihre Ästhetik wieder erkennen: der klare, helle und doch ein wenig ,wilde“ Himmel, die Inszenierung von Ernst und Arbeit, von Uninszeniertheit, vor allem das Erhabene der Froschperspektive, die das Publikum zur Hauptfigur wie zu einer Göttin oder zumindest einer "ganz Großen“ aufschauen lässt - hat sie nicht an anderer Stelle ihre Kameras buchstäblich eingegraben, um Hitler und die Seinen möglichst imposant zeigen zu können?

Das ist aber nicht der Blick der afrikanischen Deutschschülerin oder des chinesischen Studenten im Anfängerkurs. Auch fortgeschrittene Lerner werden beim Betrachten des Fotos nicht unbedingt an Aufmärsche und Konzentrationslager denken, sondern sich zum Beispiel fragen, warum auf diesem offenkundig ,alten“ Bild eine Frau die Kamera führt, wo im Film wie in so vielen anderen Berufen 
doch bis heute die Männer den Ton angeben, werden sich für ihr Aussehen, ihre Kleidung, ihre Arbeitstechniken interessieren, Geschmacksurteile äußern können und vieles mehr.

Das Titelbild ist eine historische Quelle, die ebenso gut Einleitung, Illustration oder Gegenstand einer Unterrichtsstunde oder -sequenz zu Aspekten des Dritten Reichs sein kann wie zu, sagen wir: Kleidungsstücken (also Wortschatz), Lokalpräpositionen (Grammatik), Vermutungen (mündlicher Ausdruck) oder anderem. Man sieht: Historische Quellen sind vielfach einsetzbar.

Was versteht man aber eigentlich unter einer ,historischen Quelle“? „Man hat seit jeher versucht, die Fülle der historischen Quellen durch Gliederung übersichtlicher und greifbarer zu gestalten. Um die Art, wie solche Einteilung am zweckmäßigsten und logisch am einwandfreiesten zu geschehen habe, ist in der geschichtstheoretischen Literatur eine umfangreiche und voraussichtlich auch endlose Diskussion entstanden", heißt es in Ahasver von Brandts Klassiker zum Thema (Brandt 131992: 49). Das Bemühen, eine absolute Ordnung mit ebensolcher Gültigkeit finden, erscheint ihm müßig, ,sei es nun, dass sie vom Stoff (Stein, Metall, Pergament, Papier), von der Aussageform (Gerät, Bild, Schrift, Sprachform) oder von der Zwecksetzung (,Literatur', Recht, Verwaltung) ausgeht, oder aber den Aussagewert zum Kriterium der Einteilung macht." Schließlich seien die Quellen „nicht Selbstzweck, sondern nur Mittel zum Zweck der historischen Erkenntnis“ (Brandt: 50). Es ist festzuhalten: Auch Knochen, Geräte, Steine, selbst Bauwerke sind historische Quellen.

J.G. Droysen und E. Bernheim haben folgende Unterscheidung etabliert: „Überreste: Alles, was unmittelbar von den Begebenheiten übriggeblieben ist. Tradition: Alles, was von den Begebenheiten übriggeblieben ist, hindurchgegangen und wiedergegeben durch menschliche Auffassung." In ähnlicher Weise spricht H.L. Mikoletzky von „,willkürlicher“ und ,unwillkürlicher“ Überlieferung (Brandt: 52).

Von Brandt fährt fort: „Unabsichtlich dient uns jede Quelle, die, ,unmittelbar von den Begebenheiten übriggeblieben', in ihrer Entstehung nicht den Zweck historischer Unterrichtung der Mit- oder Nachwelt verfolgt, sondern entweder aus anderer Zwecksetzung oder zweckfrei entstanden ist.“ Dazu gehören Sachüberreste wie Gebäude, Kunstwerke, Gegenstände des täglichen Bedarfs wie Gerät, Kleidung, Möbel, Waffen, Wappen, Münzen, körperliche Überreste; dann abstrakte Überreste wie Institutionen Rechts- und Verfassungszustände in mündlicher Überlieferung, Sitten und Gebräuche, Sprachen und Sprachformen, Ortsund Flurnamen und vieles mehr; schließlich ,das gesamte Schriftgut, das aus geschäftlichen oder privaten Bedürfnissen der jeweiligen Gegenwart entstanden ist, dessen Zweck mithin eine geschäftliche, rechtliche, politische, wirtschaftliche oder persönlich-private Dokumentation ist" (Brandt: 53) - und was sich also in Archiven befindet.

„Tradition“ sind dagegen im weitesten Sinne ,literarische“ Quellen, und zwar sowohl „primär mündlich überlieferte, wie Mythen, Sagen, historische Lieder, 
genealogische oder historische Erzählungen“ wie die schriftlich fixierten, z.B. „Annalen, Chroniken, Biographien, Memoiren, Autobiographien, zeitgenössische Geschichtsdarstellungen aller Art.“ Auch ein großer Teil der Publizistik sei hierzu zu rechnen (Brandt: 54).

Die Beiträge des vorliegenden Bandes gehen größtenteils auf eine DAADFachtagung an der Université de Picardie Jules Verne (UPJV) Amiens im März 2011 zurück. Ihr lag eine simple Überlegung zugrunde: Im Sprach- und Landeskundeunterricht sind Medien unerlässlich. Gegenüber den vorgefertigten Produkten der einschlägigen Verlage und Internetplattformen haben historische Quellen aber einen großen Mehrwert, indem sie als Lern- und im weiteren Sinne Bildungsmedien mit Wiedererkennungswert gewissermaßen en passant Kultur vermitteln und Anknüpfungspunkte an landeskundliche und medienwissenschaftliche Themen bieten.

Die Artikel der Beiträger zeigen auf sehr überzeugende Weise, wie vielfältig die Quellen und ihre Einsatzmöglichkeiten sind; sie sprechen für sich und bedürfen also nicht der für Einleitungen durchaus üblichen Vorankündigung. Vielmehr sei hier abschließend auf einige Lücken des Bandes hingewiesen.

Literatur als historische Quelle ist Gegenstand der Kulturwissenschaften, die sich mit Dingen wie historischen Zukunftsvorstellungen, Geschlechter-, Technik-, Krankheits- und anderen Diskursen beschäftigen; jede Stoff- und Motivgeschichte nutzt Literatur als historische Quelle, indem sie etwas über die Ausformungen ihres Stoffes oder eben Motivs zu einer und durch eine bestimmte Zeit aussagt. Anders gesagt: Der Nutzen von Literatur als historische Quelle ist konstitutiv für die Literaturwissenschaft und kann schon deshalb nur bedingt Gegenstand eines Artikels im vorliegenden Rahmen sein; Jens Grimstein zeigt am Beispiel des Unterbereichs der Arbeiterliteratur zahlreiche Einsatzmöglichkeiten für den DaFUnterricht auf.

Comics werden trotz aller Anerkennung weniger „Meisterwerke“ in Feuilleton, Wissenschaft und Unterricht bis heute eher stiefmütterlich behandelt. Als einzigartige historische Bild-/Textquellen können sie auch im Geschichts-, Deutsch und DaF-Unterricht eingesetzt werden; zwei der Beiträger haben sich an anderer Stelle eingehend mit dem Thema beschäftigt (Sistig 2002; Hieronimus 2009; 2010).

Auch Zeitungen sind eine wichtige und gut einsetzbare Quelle, zunächst für Stimmungslagen, nicht zuletzt aber auch durch ihre privaten und gewerblichen Anzeigen (vgl. z.B. Hieronimus 2006). Das Projekt der „Zeitungszeugen“, also der kommentierten Wiederveröffentlichung von Zeitungen aus der NS-Zeit inklusive Werbung und Propagandaposter, zeugt durch seinen Erfolg vom anhaltenden Interesse für diese Quellenart. Ein interessanter Nebenaspekt der Arbeit mit diesen und allgemein Schriftquellen wie Urkunden und Briefen ist die Begegnung mit alten, dem ungeübten Leser nicht unmittelbar zugänglichen Hand- und Druckschriften wie Sütterlin oder Fraktura.

Darüber hinaus liegt es im Ermessen und Interesse jeder Lehrkraft, auch gegenständliche Quellen als Anschauungs- und Lehrmaterial zu verwenden, handele es sich 
nun um Münzen, Kleidung und andere Dinge des Alltags, fachspezifische Gegenstände wie etwa Werkzeug und stilgeschichtlich Interessantes aus der Angewandten Kunst, oder Abbildungen repräsentativer und also mehr oder minder berühmter Artefakte der europäischen Geschichte. Eins möge der geneigte Leser davontragen: Man kann nicht nur über alles reden - man kann auch mit allem unterrichten!

Der Band 86 der Reihe MatDaF konnte nur durch die Mitarbeit und Unterstützung zahlreicher Personen und Institutionen zustande kommen. Der Dank des Herausgebers gilt in chronologischer Reihenfolge dem DAAD und der UPJV für die finanzielle und logistische Unterstützung; den Beiträgern für ihre spannenden Vorträge und fast sämtlich fristgerecht eingereichten Beiträge; dem $\mathrm{FaDaF}$ und besonders Annegret Middeke und Annett Eichstaedt für die geduldige Betreuung des Buchprojekts; und nicht zuletzt Bertrand Delville für die zuverlässige Überwindung aller technischen Hindernisse.

\section{Literatur}

Brandt, Ahasver von (131992): Werkzeng des Historikers. Stuttgart, Berlin, Köln: Kohlhammer.

Hieronimus, Marc (2006): Krankbeit und Tod 1918. Vom Umgang mit der Spanischen Grippe in Frankreich, England und dem Deutschen Reich. Münster: Lit-Verlag.

Hieronimus, Marc (2009): Geschichtscomics im DaF-Unterricht. In: Nieradka, Magali Laure; Specht, Denise (Hrsg.) (2009): Fremdkörper? Aspekte der Geisteswissenschaften in der Auslandsgermanistik und im DaF-Unterricht. Münster: LitVerlag, 101-114.

Hieronimus, Marc (2010): Hitler is fun. Sixty Years of Nazism in Humorous

Comics. In: Buttsworth, Sara; Abbenhuis, Maartje (Hrsg.) (2010): Monsters in the Mirror. Representations of Nazism in Post-W ar Popular Culture. Westport, Connecticut: Praeger, 75-100.

Sistig, Joachim (2002): Invasion aus der Vergangenheit. Das Deutschlandbild in frankophonen Bandes Dessinées. Frankfurt/Main: Peter Lang. 


\title{
Deutsche Geschichte im DaF-Unterricht - zur Arbeit mit historischen Quellen
}

\author{
Uwe Koreik
}

\section{Landeskunde und Geschichte im DaF-Unterricht}

Die Vermittlung historischer Themen im DaF-Unterricht - insbesondere auch wenn es um den Einsatz historischer Quellen geht - ist im Kontext der Diskussion um die Landeskunde und die Landeskundevermittlung sowie ihren Anteil und ihre Bedeutung für den Unterricht in Deutsch als Fremdsprache zu verorten. „Es ist seit langem schon unbestritten, dass Sprachvermittlung immer auch Kulturvermittlung bedeutet, weil das Erlernen einer Sprache immer auch zugleich den $\mathrm{Zu}$ gang zu einer neuen Welt, anderen Werten und Wertvorstellungen, anderen Einstellungen und Verhaltensweisen eröffnet. Für die Vermittlung kultureller Inhalte über Land und Leute des Zielsprachenlands hat sich seit den 1960er Jahren im Sprachunterricht und in der Sprachlehrerausbildung als Begriff Landeskunde durchgesetzt und bis heute hartnäckig gehalten" (Koreik 2011). Dabei verdeutlicht die Tatsache, mit welchen unterschiedlichen Begriffen der kulturkundliche Vermittlungsbereich innerhalb der Philologien seit dem Ende des 19. Jahrhunderts bezeichnet wurde (Realienkunde, Kulturkunde, Landeskunde, Landeswissenschaft, Kulturstudien u.a., vgl. Koreik; Pietzuch 2010, Altmayer; Koreik 2010) zugleich, welche unterschiedlichen Kernkonzepte damit jeweils auch intendiert waren. Von der reinen Faktenvermittlung (gerne polemisch als „Stadt, Land, Fluss“ charakterisiert) über den kommunikativen Ansatz, bei dem Alltagsverhaltensweisen im Vordergrund standen, bis zum interkulturellen Anspruch seit den späten achtziger Jahren, bei dem mit dem Ziel, sich und andere besser zu verstehen, eine kulturelle 
Uwe Koreik

Kompetenz angestrebt wird, reichen die großen Trendlinien, die zumeist jedoch in vermischten Formen auftraten und auftreten. Immer jedoch spielte das Thema „Geschichte" bei der Vermittlung kultureller Inhalte eine mehr oder weniger große Rolle. $^{1}$

\subsection{Ziele und Bedingungsfaktoren bei der Vermittlung historischer Themen}

„Landeskunde ist in hohem Maße auch Geschichte im Gegenwärtigen“ heißt es in den ABCD-Thesen (1990: 307). Daraus ergebe sich „die Notwendigkeit, auch historische Themen und Texte im Deutschunterricht zu behandeln. Solche Themen sollten Aufschluß geben über den Zusammenhang von Vergangenheit, Gegenwart und Zukunft, über unterschiedliche Bewertungen sowie über die Geschichtlichkeit der Bewertung selbst" (ebd.). In drei Sätzen sind damit sehr hohe und komplexe Ansprüche formuliert, die sich je nach Zielgruppe und dem gegebenen sprachlichem Niveau nur begrenzt werden einlösen lassen. Gerade auch bei der Einbeziehung historischer Themen in den Unterricht in Deutsch als Fremd- bzw. -Zweitsprache spielen zahlreiche Faktoren wie Lernort, Vermittlungsinstitution (und ihre Lehrziele bzw. die Curricula), Vorbildung, Zusammensetzung der Lernergruppe (im Hinblick auf Alter, Nationalität, Geschlecht), die Motivation für den Spracherwerb, die vorhandenen Medien, die Qualifikation und auch die Begeisterungsfähigkeit der Lehrenden eine bedeutende Rolle.

Die Frage nach dem Gegenwartsbezug hat der Historiker Heinrich-August Winkler, natürlich nicht mit Bezug auf den DaF-/DaZ-Unterricht, sondern mit Blick auf die Geschichtswissenschaft kürzlich folgendermaßen beantwortet: „Die entscheidende Frage der Geschichtswissenschaft lautet aus meiner Sicht nicht so sehr, frei nach Ranke, wie es eigentlich gewesen ist, als vielmehr, warum es eigentlich so gekommen ist. Das heißt, wir sollten auch fragen, wie unsere Gegenwart entstanden ist, warum sich unsere Geschichte so und nicht anders entwickelt hat" (Winkler 2011). Die gleichen Fragestellungen sind auch für den DaF-/DaZ-Unterricht relevant.

In der zitierten ABCD-These (vgl. Koreik 2010: 1478-1479), die sich in ihrem Aussagegehalt in zahlreichen späteren Veröffentlichungen im Fach widerspiegelt, wird nicht nur auf die Bedeutung des Zusammenhangs von Vergangenheit und Gegenwart verwiesen, sondern ebenfalls ein geradezu prognostisch anmutender Blick in die Zukunft gefordert sowie vor allem eine Verständnissicherung für die Relativität der Bewertung geschichtlicher Ereignisse und Prozesse erwartet. Der gewagten Forderung nach dem Blick in die Zukunft verweigern sich in der Regel die Historiker, sondern beziehen meist ähnlich wie Winkler (2011) in diesem Fall mit Verweis auf Schlegel Position: „Zunächst einmal hat Friedrich Schlegel wahr-

1 Zum Thema Geschichte in DaF-Lehrwerken: Koreik 1995; Koreik 2010; Maijala 2004; Maijala 2007; Thimme 1994; Thimme 1996. 
scheinlich recht mit seinem berühmten Bonmot, dass die Historiker rückwärts gekehrte Propheten sind. Für mich ist die Frage weniger, was kommen wird, sondern, auf welche Zukunft wir hinarbeiten sollten" (ebd.).

Aus der Geschichte lässt sich zwar vielleicht Einiges lernen - der Umgang mit der Weltwirtschaftskrise in der ausgehenden ersten Dekade des 21. Jahrhunderts zeigt, dass zumindest zunächst in gewisser Weise aus den Erfahrungen von 1929 gelernt wurde - jedoch einen konkreten Zusammenhang zur Zukunft herzustellen, ist eben nicht nur eine Forderung, der sich die Fachwissenschaftler verweigern, sondern auch eine Aufgabe, deren Umsetzung im Sprachunterricht allenfalls Gesprächsanlässe - gelegentlich allerdings sogar sehr interessante - bieten dürfte, welche lediglich zu Spekulationen einladen.

Die Relativität geschichtswissenschaftlicher Darstellungen hingegen hat nicht nur einen hohen erkenntnistheoretischen Stellenwert, sondern ist zudem je nach Zusammensetzung der Lernergruppe von einiger Brisanz, da Lerner nicht selten aus Lernkulturen kommen, in denen Geschichtskenntnisse weitgehend unhinterfragt als positivistisch gesichertes Wissen vermittelt werden. Dabei ist eindeutig, „,...] dass Geschichte immer wieder aus einem bestimmten Blickwinkel wahrgenommen und überliefert wird“ (Sauer ${ }^{72008: ~ 17) . ~ U n d ~ d i e s e r ~ B l i c k w i n k e l ~ k a n n ~}$ zunächst auch ein national vermittelter sein, bei dem Schulbücher, traditioneller Geschichtsunterricht und die jeweilige mediale Vermittlung das Geschichtsbild prägen.

Die Auswertung neu zugänglicher Quellen, neue oder anders gewichtete geschichtswissenschaftliche Ansätze und ein damit sich verändernder Blickwinkel sowie auch jeweils vorherrschende gesellschaftspolitische Strömungen führen nicht selten dazu, dass historische Ereignisse und Prozesse neu bewertet werden und sich eine andere Lehrmeinung durchsetzt, wie beispielsweise die Nachwirkung der „Fischer-Kontroverse“ um das Ausmaß der deutschen Schuld am Ausbruch des 1. Weltkriegs deutlich gezeigt hat. Eine derartige Meta-Ebene der Betrachtung lässt sich im Landeskundeunterricht allenfalls in Ansätzen realisieren, allerdings kann „[m]ithilfe multiperspektivischer Zugänge (bei der Auswahl der Themen, bei der Auswahl und Bearbeitung historischer Quellen, bei der Bewertung von Ereignissen, ...) [versucht werden] historische und gegenwärtige Konflikte von verschiedenen Seiten zu betrachten und sich dabei der Relativität der eigenen (nationalen, geschlechtsspezifischen, sozialen, ...) Sichtweise bewusster zu werden" (Grabe 2004: 25).

Als Zielsetzung für die Vermittlung historischer Inhalte, durchaus auch auf den DaF-/DaZ-Kontext übertragbar, formuliert Winkler sehr klar: „[...] nicht die Anhäufung von Wissen ist das, was wir den Studierenden vermitteln sollten, sondern die Erkenntnis von Zusammenhängen, die Fähigkeit, einzelne Erscheinungen in einen größeren Kontext einzuordnen. Das setzt in allererster Linie Problemverständnis voraus, die Fähigkeit, nach Motiven, Handlungsspielräumen und Alternativen der Akteure zu fragen. Das ist die Grundlage jedes historischen Urteils. Das - 
Uwe Koreik

glaube ich - ist ein Zugang zur Geschichte, der uns auch offen macht für die Herausforderungen der Gegenwart" (ebd.).

Für die Einbettung historischer Themen in den DaF-/DaZ-Unterricht, in dem Sprachvermittlung immer auch eine gewisse Rolle spielt, gilt es bestimmte Voraussetzungen zu beachten bzw. sie zu schaffen.

Einmal abgesehen davon, dass die gewählten Themen möglichst einen Aktualitätsbezug aufweisen (häufig lassen sich Entwicklungslinien herstellen) oder einen Erkenntniswert vermitteln sollten, der im Kontext zur Gesamtausbildung der Lernergruppe steht, gilt es bei der Auswahl der Texte das sprachliche Niveau der Lernergruppe im Blick zu haben. Der Schwierigkeitsgrad der Texte - und das gilt insbesondere bei historischen Quellen - darf das durchschnittliche Sprachvermögen der Lernergruppe nur begrenzt übersteigen. Eine veraltete Lexik, Begriffe mit einer speziellen zeitgebundenen Konnotation sowie syntaktische Besonderheiten, die nicht mehr der aktuell verwendeten deutschen Sprache entsprechen, erschweren nicht nur das Textverständnis, sondern werfen auch die Frage auf, welcher Gewinn für die Sprachausbildung erreicht werden kann. Diese Frage wird unterschiedlich je nach Lernergruppe zu beantworten sein. Handelt es sich beispielsweise um eine Gruppe Studierender an einer ausländischen Hochschule, die als Studienfach auch Geschichtswissenschaft hat, wird man kaum Grenzen ziehen wollen; handelt es sich jedoch um Studierende, die die deutsche Sprache „lediglich“ im Rahmen einer Zusatzqualifikation neben weniger affinen Studienfächern erlernen wollen, gilt es für die Lehrkraft, eine Güterabwägung zu treffen und die Materialauswahl im Zweifelsfall zugunsten der sprachlichen Weiterverwendbarkeit zu treffen. Werden mehrere historische Quellentexte in einer Reihe hintereinander behandelt, gilt es nach Möglichkeit auf die inhaltliche und sprachliche Progression zu achten, bei der im Idealfall auf beiden Ebenen schrittweise das Anspruchsniveau gesteigert wird.

Die häufig vorgebrachte Forderung nach einer kontrastiven oder gar interkulturellen Perspektive lässt sich bei historischen Themen nicht selten leicht einlösen, weil es in der Weltgeschichte immer wieder eine Ungleichzeitigkeit des Ähnlichen gibt, was bedeutet, dass historische Ereignisse und Prozesse immer auch im Zusammenhang mit vergleichbaren Abläufen zu anderen Zeiten in anderen Ländern oder gar Kulturkreisen betrachtet werden können. Eine derartige Vorgehensweise ist allerdings sehr anspruchsvoll, und das Problem dabei ist dann meistens die mangelnde Kompetenz Lehrender wie Lernender (vgl. Koreik 1998).

In den bereits zitierten ABCD-Thesen heißt es an anderer Stelle: „Primäre Aufgabe der Landeskunde ist nicht die Information, sondern Sensibilisierung sowie die Entwicklung von Fähigkeiten, Strategien und Fertigkeiten im Umgang mit fremden Kulturen. Damit sollen fremdkulturelle Erscheinungen besser eingeschätzt, relativiert und in Bezug zur eigenen Realität gestellt werden. So können Vorurteile und Klischees sichtbar und abgebaut sowie eine kritische Toleranz entwickelt werden“ (ebd.). Wenn man einmal von der hehren Hoffnung ein wenig Abstand nimmt, dass sich Vorurteile und Klischees, wenn sie denn sichtbar gemacht wurden, auch leicht abbauen ließen, bleibt die Diskrepanz zwischen Infor- 
mationsvermittlung und Sensibilisierung bzw. Entwicklung von Fähigkeiten, Strategien und Fertigkeiten. Damit wird 1990 ein Trend festgeschrieben, der als Hintergrund die Erkenntnis hat, dass sich die Totalität der gesellschaftlichen Wirklichkeit sowieso nicht im landeskundlichen Unterricht erfassen bzw. abbilden und vermitteln lässt, was auch auf die Vermittlung von Geschichtsthemen zutrifft. Im Sprachunterricht kann keineswegs die gesamte deutsche Geschichte nebenbei vermittelt werden, und auch in speziellen Landeskundeveranstaltungen zur deutschen Geschichte, wie es sie in manchen Ländern nach wie vor im Rahmen des Germanistikstudiums gibt, kann die Geschichte von den Germanen bis ins 20. Jahrhundert nur in Form meist eher oberflächlichen Faktenwissens vermittelt werden, wobei häufig die Ereignisgeschichte mit den Aspekten Politik-, Diplomatie- und Militärgeschichte im Vordergrund steht und wichtige Themenstränge der Wirtschafts- und Sozialgeschichte in der Regel eher vernachlässigt werden. Selbst für den schulischen Geschichtsunterricht in der Bundesrepublik Deutschland heißt es: „Die vollständige Behandlung der Geschichte ist weder möglich noch wünschenswert. Die Zahl der Themen ist prinzipiell unendlich und es kommt auf begründete Auswahl an“ (Sauer 72008: 19).

Das spätestens seit der Veröffentlichung der ABCD-Thesen häufig propagierte Ziel ist es deswegen vielmehr, stärker themenorientiert und exemplarisch zu arbeiten, gleichzeitig damit die Wahrnehmung und Reflektion zu schulen und dabei die traditionelle Faktenvermittlung deutlich in den Hintergrund treten zu lassen. Der Tendenz, die Wissensvermittlung als völlig untergeordnetes Ziel im Fremdsprachenunterricht zu sehen, ist jedoch Schücking bereits 1927 mit folgenden Argumenten entgegen getreten: „Es klingt sehr gut: Nicht Kenntnis, sondern Erkenntnis! Nicht Wissen, sondern Fähigkeit zur Fragestellung! Aber Erkenntnis setzt zunächst einmal eine gewisse Kenntnis, die Fähigkeit zur Fragestellung setzt auch Wissen voraus“ (Schücking 1927: 10). Galt lange Zeit die Vermittlung von Wissensbeständen über Land und Leute im DaF-/Daz-Unterricht in der Landeskundediskussion seit der „kommunikativen Wende“ als verpönt und wurde als reine Faktenkunde gebrandmarkt, auch wenn diese Art des Landeskundeunterrichts in weiten Teilen der Welt - auch bis ins Extreme gesteigert - weiter betrieben wurde, so ist inzwischen eine Rückbesinnung auf den Wert von auch Informationen vermittelndem, Hintergründe beleuchtendem und nicht nur rein kommunikativ ausgerichtetem Landeskundeunterricht erfolgt. Für DaF-Lehrwerke aus Estland, Finnland, Frankreich, Großbritannien und Norwegen konstatiert Minna Maijala die „Rückkehr der Geschichte in die europäischen Deutschlehrwerke“ und stellt zugleich fest, „dass der kognitive Ansatz der Landeskunde in den Mittelpunkt des Fremdsprachenunterrichts gerückt ist" (Maijala 2004: 345). Weitgehend auf Faktenvermittlung ausgerichtete Geschichtskapitel sind in Deutschland seit 2005 in speziellen Lehrwerken für Deutsch als Zweitsprache entstanden, die speziell in Integrationskursen eingesetzt werden. Einer der vier Themenschwerpunkte lautet „Überblick über die jüngere deutsche Geschichte“, wobei bis jetzt allerdings nicht umfassend untersucht worden ist, inwieweit damit tatsächlich eine Horizonterwei- 
Uwe Koreik

terung erreicht wird, oder - wovon leider auszugehen ist - ob nur Fakten für den anschließenden Orientierungstest auswendig gelernt (und dann bald auch wieder vergessen) werden.

Ein anderer viel versprechender Ansatz wird in dem von Schmidt und Schmidt (2007) herausgegebenen Lehrwerk „Erinnerungsorte. Deutsche Geschichte im DaF-Unterricht“" verfolgt. In Anlehnung an das Konzept des französischen Historikers Pierre Nora finden sich hier zu dreizehn deutschen „Erinnerungsorten“ Unterrichtseinheiten, welche ,exemplarisch Wege in und durch die deutsche Geschichte [eröffnen]" und als Zusatzmaterialien für den Sprach- und Landeskundeunterricht konzipiert sind (Schmidt; Schmidt 2007: 6).

Die zwei dem Buch beigefügten CD-Roms bieten eine Fülle an Zusatzmaterialien - auch Hörtexte, sowie historisches Quellenmaterial - und ermöglichen eine vielschichtige Herangehensweise, wie sie für den Einsatzort Bulgarien in mustergültiger Weise und mit der Möglichkeit des Perspektivenwechsels (Fornoff 2009) ausgearbeitet wurde. In einem modernen in den Sprachunterricht integrierten Landesunterricht, in dem historische Themen behandelt und die Arbeit mit historischen Quellen in den Vordergrund geraten, werden auch in Zukunft mehrere Faktoren Berücksichtigung finden müssen: Adressatenspezifik und Lehrziel/Curricula-Bezug sowie Prüfungs- und Testorientierung, wenn sie institutionell vorgesehen ist. Es wird immer wieder eine Gratwanderung zwischen einem stärker informierenden, einem kommunikativ ausgerichteten, gar zur Lernerautonomie hinführenden, einem auf Interaktion setzenden und auf interkulturelle Lernziele hinarbeitenden Unterricht geben, dessen Schwerpunktsetzung, abgesehen von institutionellen Vorgaben, maßgeblich von der jeweiligen Lehrkraft vorgenommen werden muss (vgl. Koreik 2011).

Auch wenn seit den 1970er Jahren in der deutschen Geschichtsdidaktik für die Schule die Arbeit mit historischen Quellen im Mittelpunkt steht und sie sich auch für die Vermittlung historischer Themen im DaF-/DaZ-Unterricht alleine wegen ihres Authentizitätscharakters und des darin enthaltenen Lernpotentials anbietet, gilt auch für den Sprachunterricht zu historischen Themen: „Geschichtsunterricht kann nicht nur aus Quellenarbeit bestehen. Wer sie betreiben will, braucht fast immer, zu Beginn oder im Laufe seiner Untersuchung ein Vorwissen, einen bestimmten Rahmen historischer Kenntnisse“ (Sauer ${ }^{72008: ~ 110) . ~}$

\section{Zur Arbeit mit historischen Quellen im Sprachunterricht}

Opgenoorth und Schulz (62001: 40) greifen in ihrer „Einführung in das Studium der Neueren Geschichte“ zur Klärung des Begriffs „Quellen“ die viel zitierte Definition von Kirn auf: „Quellen nennen wir alle Texte, Gegenstände oder Tatsa- 
chen, aus denen Kenntnis der Vergangenheit gewonnen werden kann“,, um angesichts dieser weit gefassten Definition weitere Erklärungen und Differenzierungen vorzunehmen. Nachvollziehbar erläutern sie, warum z.B. erhaltene Kleidung aus den letzten Jahrhunderten uns als Gegenstände die Bedingungen der jeweiligen Zeit besser verdeutlichen als überlieferte Beschreibungen oder Abbildungen auf Gemälden, oder dass fremdsprachliche Bestandteile in der deutschen Sprache als Tatsachen (z.B. Italienisch in der Fachsprache der Musik im 17. und 18. Jahrhundert bei Bach oder Haydn) Zeitumstände verdeutlichen.

Wichtig auch für den Sprachunterricht ist die auch von ihnen vorgenommene Unterscheidung in „Primär-“ und „Sekundärquellen“ (41), die sich grob damit erklären lässt, dass Quellen, die Bezug auf eine ihr zugrunde liegende Quelle nehmen, als Sekundärquellen bezeichnet werden. Erst wenn das ursprüngliche Quellenmaterial nicht mehr vorhanden oder rekonstruierbar ist, bekommt eine Sekundärquelle, in der Ursprungsmaterial verarbeitet ist, zumindest partiell den Charakter einer Primärquelle.

Eine weitere Unterscheidungsmöglichkeit bietet das Begriffspaar „Quellen/Literatur“, welches bei Opgenoorth; Schulz (41) am Beispiel der „Westfälischen Friedensverträge von 1648“ und Dickmanns Buch „Der westfälische Friede“ verdeutlicht wird. Während der Vertragstext eine Quelle darstellt, ist Dickmanns Darstellung eine Interpretation und damit Literatur. Letztlich ist die Unterscheidung aber abhängig von der wissenschaftlichen Fragestellung. Will man die Positionen deutscher Historiker zu den Westfälischen Friedensverträgen Ende der 1950er untersuchen, wird Dickmanns Erstauflage von 1959 zur Quelle.

Zunächst hilfreich ist auch die traditionelle Unterscheidung in „Tradition“ und „Überrest“, wenn man sich vor Augen führt, dass Quellen, die entstanden sind, um gezielt der Nachwelt Kenntnisse zu übermitteln (z.B. Erlebnis- oder Reiseberichte o.̈.), anders zu werten sind als Quellen, die ohne diese Intention entstanden sind (z.B. Rechnungen, Inventarlisten u.a.). Aber auch diese Kategorisierung der Quellen ist nicht trennscharf, sondern „hat einen Schönheitsfehler“ (Opgenoorth; Schulz: 43), da der Begriff „Überrest“ insofern als der weitere Begriff zu werten sei, als auch Traditionsquellen den Charakter von Überresten bekämen, wenn weniger der Inhalt als vielmehr die Gegebenheiten der Entstehung in den Blick gerückt würden. Erst recht seien die Grenzen seit dem Beginn der Neuzeit zunehmend verschwommen, als Quellen insbesondere aus dem Bereich der Publizistik zum Einen als Berichterstattung für die Zeitgenossen diente (also Überrest), zum Anderen aber auch als Überlieferung gesellschaftspolitischer Sachverhalte (also Tradition). Deutlicher noch urteilt Schulze (21991: 33) über die traditionelle Einteilung in Tradition und Überrest: „Ich halte die Unterscheidung für ebensowenig sinnvoll wie die Unterscheidung willkürlicher und unwillkürlicher Überlieferung. Sie kann sogar gefährlich sein, weil solche Einteilungen möglicherweise die weitere

2 Einen informativen und prägnanten Überblick über Quellenarten bietet http://www.unikonstanz.de/FuF/Philo/Geschichte/Tutorium/Themenkomplexe/Quellen/Quellenarten/quellenarten.html. 
Nutzung präjudizieren können. Vielmehr muß gelten, daß alle Quellen den gleichen kritischen Verfahren unterzogen werden müssen, um sie zum Sprechen zu bringen. Die innere und äußere Kritik muß unbeeinflußt von a priori-Kategorisierungen angewendet werden.“

\subsection{Textquellen}

Als Textquellen benennt Sauer (2008: 187): „Akten, Annalen, Aufrufe, Augenzeugenberichte, Biografien, Briefe, Chroniken, Dramen, Flugblätter, Epen, Gedichte, Gesetzestexte, Grabinschriften, Lebensbeschreibungen, Legenden, Memoiren, Memoranden, Protokolle, Reden, Reiseberichte, Romane, Tagebücher, Urkunden, Verträge, Zeitungen" und weist darauf hin, dass die Liste keineswegs erschöpfend sein müsse. Nimmt man Textquellen der jüngsten Geschichte hinzu, müssen Begriffe wie Blog, Twitter, Facebook, E-Mails, SMS, Google-Protokolle (Wikileaks) u.a. fallen.

Bei der Arbeit mit Textquellen im DaF-/DaZ-Unterricht stehen zunächst die gleichen Grundfragen im Vordergrund wie sie in der Geschichtsdidaktik benannt werden (vgl. Sauer 72008: 190):

Verfasser/in des Texts (Amt, Stellung, soziale Schicht, Beziehung zum erwähnten Ereignis, Kenntnisstand)

Entstehung der Quelle (Wann, wo, weshalb wurde der Text verfasst?, zeitlicher Abstand zum Ereignis?

Quellengattung (Art, welche Aussagen kann man erwarten?, Art der Verbreitung des Texts)

Thema und Aussage (welches Ereignis wird erwähnt und was wird mitgeteilt)

Adressaten und Intention (wer wird angesprochen, warum, mit welcher Absicht, aus welcher Perspektive wird geschrieben, Bericht?, Urteil?, Argument? Was soll bei den Adressaten erreicht werden?

Darstellungsweise (sprachliche und rhetorische Mittel)

Gerade der letzte Punkt ermöglicht im Sprachunterricht - wenn passend und gewünscht - eine intensivere Befassung mit der Sprache selbst, wenn man sich die dazu gehörigen Unterpunkte bei Sauer (190) vergegenwärtigt: „[...] Satzgestalt (Satzart, -länge, -stellung), allgemeine Wortwahl (alltäglich, gewählt, konventionell), Argumente, Begriffe und ihre besondere Bedeutung, traditionelle Muster (Topoi), rhetorische Mittel (Symbole, Allegorien, Metaphern, Wiederholungen, Wortspiele, Lautmalereien usw.) “. Allein hierdurch dürfte klar geworden sein, dass die Arbeit mit historischen Quellen im DaF-/DaZ-Unterricht recht anspruchsvoll ist.

Gleichzeitig ist jedoch auch deutlich, dass die Quelle alleine letztlich nicht ausreicht, um Lernern eine ausreichende Interpretation zu ermöglichen. Informationen über den/die Verfasser/in, Überblickswissen über den Zeitkontext und ggf. 
Auskünfte über eine spezielle Textgattung (z.B. Flugblatt) sind erforderlich, um eine angemessene Aufschlüsselung und Einordnung einer historischen Quelle zu ermöglichen. Dabei gilt es zu berücksichtigen, dass Lerner der deutschen Sprache nicht immer eine schulische Sozialisation durchlaufen haben, in der eine textkritische Auseinandersetzung mit historischen Quellen eingeübt worden ist. Umso erhellender kann allerdings eine derartige Auseinandersetzung mit historischen Quellen im Sprachunterricht ausfallen.

Für den Einsatz historischer Schriftquellen im DaF-/DaZ-Unterricht darf man sich auch durchaus vor Augen führen, welche didaktischen Einsatzmöglichkeiten für den schulischen Geschichtsunterricht in Deutschland diskutiert werden (vgl. Sauer 72008: 191f): die Präsentation einer Textquelle in unsystematisch angeordneten Teilstücken, die erst durch genaues Lesen in eine kohärente Reihenfolge gebracht werden können, das Weglassen des Schlusses einer Textquelle, die von den Lernern sinnvoll ergänzt werden soll, zunächst fehlende Informationen über den/die Verfasser/in der Textquelle, um einen geschärften Blick für die direkten und ggf. indirekten Intentionen des Textes zu erarbeiten, oder das Weglassen aller Angaben zur historischen Situation, um auf der Basis von Vorkenntnissen und genauem Lesen die Rekonstruktion des historischen Kontextes einzufordern. Noch kreativer sind Sauers Vorschläge zur Manipulation der Textquelle: Das Einfügen von Anachronismen, die es herauszufinden gilt, das Versehen des Textes mit Lücken oder Schwärzungen, um durch die dann nötigen Ergänzungen die Textlogik nachzuvollziehen, oder das Einfügen von ,gefälschten“ Textpassagen, um zu erarbeiten, mit welchen Intentionen „Fälscher" nachträglich den historischen Text verändert haben. $\mathrm{Ob}$ man im $\mathrm{DaF}-\mathrm{DaZ}$-Unterricht zu derartigen kreativen, für viele sicherlich zu ,verspielten“ Methoden greift, wird sicherlich sehr von der Lernergruppe abhängen, wie auch von der Bereitschaft der Lehrkraft, auch - z.B. im Rahmen universitärer Veranstaltungen - unkonventionelle Wege zu beschreiten. Auf jeden Fall können strukturierende Aufgaben wie die Gliederung des Textes in einzelne Abschnitte, das Einfügen von Teilüberschriften oder die Markierung von Schlüsselwörtern hilfreiche Arbeitsschritte sein, um eine Textquelle zusammenfassend (mündlich oder auch schriftlich) auszuwerten, wobei abschließend ohne eine entsprechende Kontextualisierung nicht auszukommen sein wird.

\subsection{Tonquellen}

Für zahlreiche Ereignisse der neueren Geschichte stehen auch relativ leicht erhältliche Tondokumente zur Verfügung 3 (z.B. zur NS-Zeit oder zur Geschichte der

\footnotetext{
3 Tondokumente für verschiedene Phasen der deutschen Geschichte finden sich beispielsweise auf den Seiten von LeMO (Lebendiges virtuelles Museum Online), einem Gemeinschaftsprojekt des Deutschen Historischen Museum (DHM) in Berlin, des Hauses der Geschichte der Bundesrepublik Deutschland (HdG) in Bonn und des Fraunhofer Instituts für Software- und Systemtechnik (ISST) in Berlin. (http://www.dhm.de/lemo/home.html). Vgl. auch den Beitrag von Beatrice Wiegand im vorliegenden Band.
} 
BRD und der DDR). Diese historischen Tonquellen können selbstverständlich isoliert eingesetzt werden, haben dann allerdings auch den Charakter von Hörverstehensübungen, wobei manche Reden mehrfach gehört werden müssen, um wirklich vollständig verstanden zu werden. Diese Tondokumente können allerdings auch im Zusammenhang mit den bereits anhand einer Transkription erarbeiteten Texten eingesetzt werden und verstärken so das Authentische bei der Arbeit mit historischen Quellen.

\subsection{Bildquellen}

Von besonderer Bedeutung bei der Arbeit an historischen Themen im Sprachunterricht sind Bildquellen, weil sie zum einen bestimmte Fertigkeiten für die Interpretation voraussetzen und keineswegs einfach für sich sprechen sowie zum anderen vielfältige Möglichkeiten der Versprachlichung ohne vorgegebene Sprachmuster bieten, folglich einen kreativen Umgang mit Sprachproduktion ermöglichen (Bildunterschrift finden, Bildbeschreibung, Bildinterpretation, Kommentar usw.). „Bilder sind nicht nur bloße, Illustration“ des bisher schon durch Texte erschlossenen Wissens. Sie stellen eine eigene Quellengattung dar und bieten einen spezifischen und eigenständigen Zugang zur wissenschaftlichen Erforschung von Geschichte" (Schlaak, o.J.). Bilder bieten zwar einen anschaulichen Zugang auch zu einem historischen Thema, präsentieren jedoch immer auch einen sehr reduzierten Ausschnitt aus zumeist sehr komplexen Vorgängen und bedürfen für ihre Interpretation in der Regel eines nicht unerheblichen Maßes an Vorarbeit zum Umgang mit Bildquellen. „Ein Missverständnis gilt es grundsätzlich zu vermeiden: Bilder, auch Fotos, dürfen nicht als Abbildungen der Wirklichkeit gelesen werden, sondern als deren Interpretation, als Auseinandersetzung des Künstlers mit der erlebten Wirklichkeit, als ,visueller Bestandteil' zeitgenössischer Diskurse" (Büttner, 2007). Dabei ist die Vielfalt der Bilder und Fotos, die gewinnbringend auch im DaF-/Daz-Unterricht eingesetzt werden können, um historische Themen zu behandeln oder einzuleiten, nahezu unüberschaubar. „Die unterschiedlichsten Bildgattungen kommen als historische Quellen in Frage. Sie lassen sich einerseits nach Bildtechniken, Präsentations- und Verbreitungsformen unterscheiden: die Malerei mit Gemälde, Wandbild oder Buchmalerei; der Holzschnitt als Buchillustration oder als Flugblatt; der Kupferstich und die Lithografie, genutzt zum Beispiel für Plakate, Bilderbögen oder Ansichtskarten; die Fotografie und - nimmt man dreidimensionale Bildwerke hinzu - die Plastik“ (Sauer 2005: 1).

Erst seit Mitte der 1980er Jahre hat sich in der Geschichtswissenschaft eine historische Bildforschung entwickelt, die aber in den letzten zehn Jahren immer wichtiger geworden und als Teilbereich der Geschichtswissenschaft wie auch im Unterricht nicht mehr wegzudenken ist (z.B. Sauer 2000; Burke 2003; Jäger 2009). Ein sehr gutes Analyse-Modell (Büttner, 2007) reicht von einer vor-ikonographischen Beschreibung über eine ikonographische Analyse bis zu hin zu den Fragen nach dem Bild als Kommunikationsmittel mit folgenden Unterpunkten: 


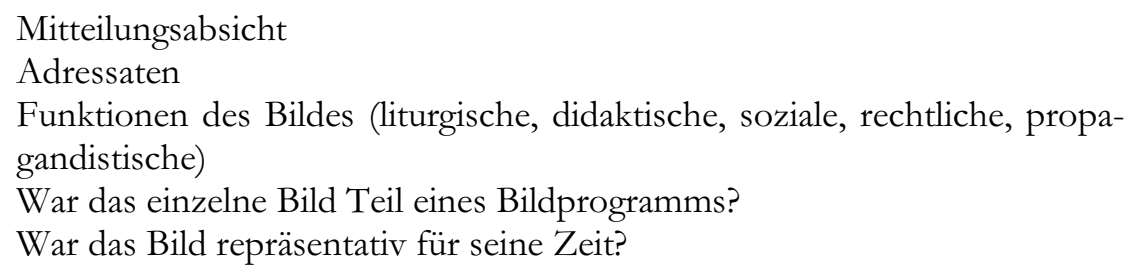

Was hier bei Büttner in historicum.net nur auf Bilder bezogen scheint, gilt in gleicher Weise für Fotos. So auch die Fragen zum wirkungsgeschichtlichen Kontext:

Wer konnte das Bild sehen?

Verbreitung?

Erwartungen und Vorkenntnisse des Bildbetrachters?

War ein Bildprogramm zu seiner Zeit erfolgreich? (weiterentwickelt / verworfen?)

Spätere Wirkung, Interpretation, Veränderung, Fälschung?

Auch wenn Bilder und Fotos als eigenständige historische Quelle zu werten und zu bearbeiten sind, können sie im DaF-/DaZ-Unterricht häufig mit Gewinn komplementär zu Textquellen eingesetzt werden. Dabei darf allerdings nicht unberücksichtigt bleiben, dass es Bilder sind, die am ehesten im Gedächtnis haften bleiben.

\subsection{Filme als historische Quellen}

Einen ganz besonderen Bereich stellt der Film als historische Quelle dar. ${ }^{4}$ Sei es der Dokumentarfilm, eine propagandistisch angelegte Wochenschau aus der NSZeit oder auch der Spielfilm, in dem Zeitströmungen transportiert werden, welche als Quellengattung eigener Art interpretiert werden können (z.B. Hey 2001). Filmmaterial entwickelt im Unterricht sehr leicht eine Eigendynamik und ist, auch wenn heutzutage alle Lerner über sehr viel Filmseherfahrung verfügen, aufgrund der Komplexität, die Lernern in der Regel nicht bewusst ist, am schwierigsten zu bearbeiten. Am sinnvollsten ist es oft - wenn zum Thema möglich -, eine Filmsequenz nach der Textarbeit ergänzend zu zeigen, um unterschiedliche Zugänge zur Interpretation eines historischen Themas zu ermöglichen.

\footnotetext{
${ }^{4}$ Eine gute Zusammenstellung der relevanten Fachliteratur (Stand 2006) findet sich von Hilke Günther-Arndt unter: http://www.historicum.net/lehren-lernen/geschichtsdidaktik/medien-historischen-lernens/visuelle-quellen-und-darstellungen/art/4_Filme/html/artikel/3003/ca/-

f1fb0e926202946b5b5032475377a21e/. Vgl. auch die Beiträge von Wolfgang Koller und Jens Grimstein im vorliegenden Band.
} 


\section{Schlussbetrachtung}

„Geschichte hat es stets mit - wie der einschlägige didaktische Begriff lautet Alteritätserfahrung, also der Erfahrung von Andersartigkeit zu tun: Wer sich mit vergangenen Zeiten befasst, begegnet dem Fremden“ (Sauer 72008: 76); so lautet die Feststellung eines deutschen Geschichtsdidaktikers. Wenn man sich die besondere Situation des DaF-/DaZ-Unterrichts vor Augen führt, in dem nichtdeutsche Lerner bemüht sind, die deutsche Sprache zu erlernen bzw. deren Handhabung zu perfektionieren und gleichzeitig Erkenntnisse über kulturelle Gegebenheiten und Hintergründe des Zielsprachenlands erfahren wollen bzw. sollen, dann ist offensichtlich, dass hier zunächst eine doppelte Verfremdung greift: die der zeitlichen wie auch die der kulturellen Fremde. Zugleich ist jedoch auch klar, dass es hinsichtlich der zeitlich bedingten Fremde die Ähnlichkeit des Ungleichzeitigen oder auch (fast) Gleichzeitigen und hinsichtlich kultureller Unterschiede die Ähnlichkeit des vermeintlich Anderen geben kann, was bedeutet, dass das Fremde gar nicht so fremd sein muss, wenn ein Bewusstsein für die jeweils eigene Geschichte (welches im Unterricht sogar herbeigeführt werden kann) den Blick auf die behandelten historischen Quellen schärft und auch erweitert.

Auf jeden Fall bietet der Einsatz historischer Quellen im DaF-/DaZ-Unterricht die Möglichkeit der Auseinandersetzung mit Authentischem, was einen Reiz an sich darstellt. Zudem eröffnet die Arbeit mit historischen Quellen eine thematische Vielfalt, die gerade auch angesichts der inzwischen im Internet zur Verfügung gestellten Quellen ${ }^{5}$ eine komfortable und flexible Unterrichtsgestaltung ermöglicht. Wird dann noch ein angemessenes Verhältnis von Beiträgen der Lehrkraft, notwendigen Hintergrundinformationen, Eigenarbeit der Lerner, zusätzlicher Recherche und nach Möglichkeit ein Zugang über verschiedene einander ergänzende Quellenarten eingehalten, kann die Arbeit mit historischen Quellen nicht nur Spaß machen, sondern ein Erkenntnispotential eröffnen, das weit über den punktuellen Erwerb von Tatsachenwissen über das Zielsprachenland hinausgeht.

\section{Literatur}

ABCD-Thesen zur Rolle der Landeskunde im Unterricht (1990): Deutsch als Fremdsprache 27(2): 306-308.

Altmayer, Claus; Koreik, Uwe: Geschichte und Konzepte einer Kulturwissenschaft im Fach Deutsch als Fremdsprache. In: Krumm, Hans-Jürgen et. al., 13771390.

\footnotetext{
${ }^{5}$ Einen guten Überblick über Quellen und Editionen bietet: http://www.historicum.net/de/lehrenlernen/internet-im-geschichtsstudium/archive-quellen-editionen/ und der Beitrag von Thomas Roth im vorliegenden Band.
} 
Büttner, Sabine (2007): Bilder als historische Quellen, in: historicum.net, URL: http:// http://www.historicum.net/lehren-lernen/arbeiten-mitquellen/bilder-als-quellen/.

Burke, Peter (2003): Augenzengenschaft. Bilder als historische Quellen. Berlin: Wagenbach.

Fornoff, Roger (2009): Erinnerungsgeschichtliche Deutschlandstudien in Bulgarien. Theoriekonzepte - unterrichtspraktische Ansätze Lehrerfahrungen. In: Informationen Deutsch als Fremdsprache 36,6, $499-517$.

Grabe, Daniela (2004): Immer dieselbe Geschichte? Konfliktbearbeitungsmethoden im DaF-Unterricht. In: Hans-Jürgen Krumm und Paul R. PortmannTselikas (Hrsg.), Theorie und Praxis. Österreichische Beiträge zu Deutsch als Fremdsprache 8, 25-38. Innsbruck etc.: Studienverlag.

Hey, Bernd (2001): Zwischen Vergangenheitsbewältigung und heiler Welt. Nachkriegsdeutsche Befindlichkeiten im Spielfilm. In: Geschichte in Wissenschaft und Unterricht 52, 228-237.

Jäger, Jens (2000): Photographie. Bilder der Neuzeit. Einführung in die Historische Bildforschung (Historische Einführungen Bd. 7). Tübingen: Edition Diskord.

Koreik, Uwe (1995): Deutschlandstudien und deutsche Geschichte. Die deutsche Geschichte im Rahmen des Landeskundeunterrichts für Deutsch als Fremdsprache. Baltmannsweiler: Schneider.

Koreik, Uwe (1998): Deutsche Geschichte und interkulturelles Lernen im Landeskundeunterricht für Deutsch als Fremdsprache. In: Wolff, Armin; Blei, Dagmar (Hrsg.): DaF für die Zukunft. Eine Zukunft für DaF!, Beiträge der 23. Jahrestagung DaF 1995. Regensburg: FaDaF, 1997, 329-340 (Materialien Deutsch als Fremdsprache, 44).

Koreik Uwe (2010): Landeskundliche Gegenstände: Geschichte. In: Krumm, Hans-Jürgen et. al., 1478-1483.

Koreik, Uwe (2011): Landeskunde. In: Ahrenholz, Bernt; Oomen-Welke, Ingelore (Hrsg.): Handbuch Deutsch als Fremdsprache (= Deutschunterricht in Theorie und Praxis, Band 10) Baltmannsweiler: Schneider (im Druck).

Koreik, Uwe; Pietzuch, Jan Paul (2010): Entwicklungslinien landeskundlicher Ansätze und Vermittlungskonzepte. In: Krumm, Hans-Jürgen et. al., 14411454.

Krumm, Hans-Jürgen; Fandrych, Christian; Hufeisen, Britta; Riemer, Claudia (Hrsg.) (2010): Deutsch als Fremd-und Zweitsprache. Ein internationales Handbuch. Berlin, New York: de Gruyter. 
Maijala, Minna (2004): Deutschland von außen gesehen. Geschichtliche Inhalte in Deutschlehrbüchern ausgewählter europäischer Länder. Frankfurt/Main: Lang.

Maijala, Minna (2007): Zur Analyse von landeskundlichen bzw. geschichtlichen Inhalten in Lehrwerken für Deutsch als Fremdsprache. In: Deutsch als Fremdsprache 44(3): 174-180.

Opgenoorth, Ernst; Schulz, Günther ('2001): Einführung in das Studium der Neueren Geschichte. Paderborn: Schöningh.

Sauer, Michael (2000): Bilder im Geschichtsunterricht. Typen, Interpretationsmethoden, Unterrichtsverfahren. München: Kallmeyer.

Sauer, Michael (2005): Bilder als historische Quellen, in: Bundeszentrale für politische Bildung, http://www.bpb.de/themen/F0RC2C.html.

Sauer, Michael (72008): Geschichte unterrichten. Eine Einführung in die Didaktik und Methodik. Seelze-Velber: Kallmeyer.

Schlaak, Alexander (o.J.): Bilder als historische Quelle, http://www.unikonstanz.de/FuF/Philo/Geschichte/Tutorium/Themenkomplexe/Quellen/ Quellenarten/Bilder/bilder.html.

Schücking, Levin L. (1927): Die Kulturkunde und die Universität. In: Die nemeren Sprachen 1, XXXV, 1-16.

Schmidt, Sabine; Karin Schmidt (Hrsg.) (2007): Erinnerungsorte. Deutsche Geschichte im DaF-Unterricht. Berlin: Cornelsen.

Schulze, Winfried (21991): Einführung in die Neuere Geschichte. Stuttgart: UTB.

Thimme, Christian (1994): Zeitgeschichte in Lehrwerken Deutsch als

Fremdsprache. In: Informationen Deutsch als Fremdsprache 21(4), 456-474.

Thimme, Christian (1996): Geschichte in Lehrwerken. Deutsch als Fremdsprache und Französisch als Fremdsprache. Ein deutsch-französischer Lebrbuchvergleich. Baltmannsweiler: Schneider.

Winkler, Heinrich-August (2011): ,Wir sind rückwärts gekehrte Propheten.“ In:

Spiegel-Online (http://www.spiegel.de/unispiegel/wunderbar/-

0,1518,751563,00.html), 32.3.2011. 


\title{
Historische Quellen im Internet
}

\author{
Thomas Roth
}

\section{Geschichte und Internet}

Die Etablierung des Internet ${ }^{1}$ in den 1990er Jahren hat auch in der Geschichtswissenschaft entgegengesetzte Reaktionen hervorgerufen: Neben kulturkritischer Abwehr und medienkritischen Kommentaren standen optimistische, zuweilen fast euphorische Einschätzungen. ${ }^{2}$ Das Internet versprach demnach:

\footnotetext{
${ }^{1}$ Der Begriff „Internet“ umfasst nicht nur Verknüpfungen im Rahmen des „World Wide Web“ (WWW), sondern zahlreiche andere Dienste (wie E-Mail, Mailinglisten, Chats, Newsgroups etc.); „Internet“ bezeichnet eher die Infrastruktur der Vernetzung, während der Begriff des ,World Wide Web" auf die abrufbaren elektronischen Dokumente verweist (s. Pscheida 2010: 9, Fn. 1). In der Folge werden beide Begriffe aus pragmatischen Gründen dennoch weitgehend synonym verwendet; wenn es um die Präsentation historischer Quellen geht, ist i.d.R. das WWW gemeint. - Stand für die in den Fußnoten genannten Internetadressen ist jeweils Juli 2011. Die zitierten Webadressen können wegen des Zeilenumbruchs zusätzliche Trennstriche enthalten.

2 Zur Diskussion um das Verhältnis von Internet, Geschichte, Geschichtswissenschaft und Geschichtsdidaktik vgl. - auch für die folgenden Ausführungen - Alavi 2010; Burckhardt; Hohls; Prinz 2007; Burckhardt; Hohls; Ziegeldorf 2005; Cornelißen 2008; Danker; Schwabe 2008a; Epple; Haber 2005; Haber; Koller; Ritter 2002; Hodel 2007; Jenks; Marra 2001; Kröll 2010; Kümper 2010; Schmale 2010; Schmale et al. 2007; Schürer 1999; Schwabe 2010; Spahn 2009; zu Internet, Erinnerungs- und Geschichtskultur Assmann 2004: 55ff.; Assmann 2006: 243ff.; Bavendamm 2006; Beier 2000; Dornik 2004; Dornik 2010; Hein 2005: 184ff.; Hein 2009a; Meyer 2009a; Meyer 2009b; Zierold 2006: 166ff. Die Literatur über Chancen, Grenzen und Gebrauchsweisen des Internet ist kaum noch überschaubar und muss hier nicht ausführlicher benannt werden. Zur mediengeschichtlichen Einführung vgl. Bösch 2011: 227ff.; Haber 2009; Hörisch 2004: 386ff.; Pscheida 2010: 11ff., 65ff., 245ff.
} 
- erweiterte Möglichkeiten wissenschaftlichen Austausches durch die Verkürzung der Kommunikationswege, die Beschleunigung der Interaktion und neue Formen wissenschaftlicher Publikation und Edition;

- bessere Forschungsbedingungen durch die Digitalisierung analoger Informationsträger und den Wegfall von institutionellen oder räumlichen Barrieren beim Zugriff auf Bibliotheken und Archive;

- erweiterte Möglichkeiten bistorischen Erzäblens durch Hypertext und Multimedia, die (nichtlineare) Verknüpfung von Texten und Kombination mit Audio-, Video- und Fotosequenzen;

- Impulse für die Geschichtsvermittlung und das gesellschaftliche Geschichtsbewusstsein durch die bessere „Anbindung“ breiter Bevölkerungskreise an historische Ausstellungen, ihre Einbindung in geschichtspolitische Debatten und Beteiligung an öffentlichen Erinnerungsprozessen;

- neue Möglichkeiten bistorischen Lebrens und Lernens durch Informationszuwächse und Interaktivität, eine verbesserte Kommunikation zwischen Lehrer und Lerner, die Auflösung streng hierarchischer Unterrichtssituationen und zielgruppenangepasste Umgebungen für individuelles, selbsttätiges, forschendes Lernen.

Wie bei allen medientechnologischen Umbrüchen (vgl. Bösch 2011; Hörisch 2004; Vowinckel 2010) hat sich auch in Bezug auf das Internet mittlerweile eine realistischere Einschätzung durchgesetzt. Die Geschichtswissenschaft sieht ähnlich wie die Fremdsprachendidaktik ${ }^{3}$ - die allgemeinen Potenziale der neuen Technologie, betrachtet aber auch die mit ihr verbundenen Probleme und beachtet nun stärker die konkrete institutionelle und individuelle Nutzung des „Netzes“. Dabei zeigt sich auch, dass die erweiterten technischen Möglichkeiten zu einer Zunahme von Daten und Veröffentlichungen, ,information overload“ und Orientierungsschwierigkeiten führen. Das Internet mag zur einer Ausdifferenzierung, Pluralisierung oder gar „Demokratisierung“ der Erinnerungs- und Geschichtskultur(en) beitragen, ${ }^{4}$ relativiert aber auch sinnvolle Strukturen zur Bewertung, kritischen Reflexion und Filterung historischen Wissens; die Vertrautheit zumal jüngerer Nutzer mit dem „Netz“ und seinen Inhalten kann nicht mit Medienkompetenz, geschweige denn mit historischer Methodenkompetenz gleichgesetzt werden. Traditionelle Vermittlungs- und Unterrichtsformen sind durch E-Learning, Weblogs und Wikis nicht entbehrlich geworden, während die Chancen, die das Internet für eine hypermediale, modulare und multiperspektivische Präsentation von historischen Sachverhalten bietet, oft (noch) nicht einmal genutzt werden. Das Internet ist also nicht die - befürchtete oder ersehnte - Maschine

\footnotetext{
${ }^{3}$ Vgl. die zahlreichen Publikationen, die seit Ende der 1990er Jahre zur Bedeutung der „,neuen“ oder „digitalen Medien“ im Fremdsprachenunterricht entstanden sind; die Diskussionen resümierend: Bausch; Christ; Krumm ${ }^{4} 2003$ : 269ff., 426ff., 430ff.; Krumm et al. 2010: etwa 1199ff., 1227 ff. sowie Huneke; Steinig 52010: $210 \mathrm{ff}$.

${ }^{4}$ So die gängigen begrifflichen Formeln in der Literatur, vgl. Anm. 2.
} 
einer neuen Geschichtswissenschaft, -didaktik oder Geschichtskultur, ${ }^{5}$ sondern Arbeitsmittel, Präsentationsplattform und Kommunikationsraum mit sehr unterschiedlichen Optionen, „Gebrauchsweisen“ (Dörte Hein) und Akteuren. Das wird sich auch beim Blick auf historische Quellen und Materialien im Internet zeigen.

\section{Historische Quellen, Digitalisierung und Internet}

Seit Ende der 1990er Jahre sind zahlreiche Initiativen zur Digitalisierung von historischen Quellen entstanden, die nicht notwendigerweise, aber in der Regel mit dem Internet verbunden sind (vgl. Burckhardt; Hohls; Prinz 2007: Bd.1, 69ff., 206ff., 466ff.; Czmiel et al. 2005; Haber 2009: 141ff., 164ff.; Hering 2006; Jahrbuch 2007; Kruse 2008: 29ff., 43ff.; Maier 2007; Sahle 2001). Zu Beginn stand die Digitalisierung von historischen Findmitteln und Bestandskatalogen im Mittelpunkt. Der weltweite Zugriff auf die Verzeichnisse von Bibliotheken und Archiven, heute eine Selbstverständlichkeit, absorbierte beachtliche finanzielle und personelle Ressourcen, so dass die Digitalisierung der Quellen selbst oft erst in einem zweiten Schritt möglich war. Dabei ging es einmal darum, bisher oft entlegene Dokumente der wissenschaftlichen „Community“ zugänglich machen und Quellen für computergestützte Analyseverfahren aufzubereiten, zum anderen um Erhalt und Datensicherung bei sensiblen Beständen oder die Präsentation von herausragendem Kulturgut. Antriebskräfte für die vermehrte Einbindung von historischen Quellen ins Internet waren allerdings nicht nur Ambitionen von Wissenschaft und Archiven, sondern auch die Bedürfnisse einer Öffentlichkeit, die sich intensiv mit geschichtlichen Ereignissen und Verantwortlichkeiten beschäftigt, in der historische Befunde und Deutungskonflikte eng mit kultureller Sinnstiftung und politischen Debatten verbunden sind und „die Geschichte“ mittlerweile ein etabliertes Unterhaltungsmittel ist. ${ }^{6}$

Ein großer Teil der heute im Internet zu findenden Quellen zur deutschen Geschichte wurde von großen staatlichen Archiven und Bibliotheken zugänglich gemacht, die - oft gefördert von Ministerien und Einrichtungen wie der Deutschen Forschungsgemeinschaft - zunächst kostbare mittelalterliche und frühneuzeitliche Urkunden, wichtige kirchen-, politik- und rechtsgeschichtliche Dokumente, Gelehrtennachlässe, schwer erreichbare Drucke des 15.-18. Jahrhunderts

\footnotetext{
5 Jüngere Untersuchungen zum World Wide Web betonen, dass sich im „virtuellen Raum“ keine eigenständige „Erinnerungskultur“ entwickle, sondern Online-Angebote andere „Erinnerungsmedien" und -angebote ergänzten und spiegelten (vgl. Hein 2009a; Hein 2009b: 164ff.; Meyer 2009b sowie Dornik 2004).

${ }^{6}$ Vgl. hierzu einführend Barricelli; Hornig 2008; Bösch 2007; Bösch; Goschler 2009; Hardtwig; Schug 2009; Korte; Paletschek 2009; Paul 2010; Schwarz 2010; zur Geschichte historischer Debatten und Auseinandersetzungen in Deutschland nach 1945 einführend Große Kracht 2005; Sabrow; Jessen; Große Kracht 2003; Wolfrum 1999; Wolfrum 2001: 56ff.
} 
oder frühes wissenschaftliches Schrifttum erschlossen ${ }^{7}$ haben. Parallel dazu sind geschichtswissenschaftliche Internet-Portale und -Journale entstanden, in denen gelegentlich historische Dokumente präsentiert, quellenkritisch eingeordnet sowie geschichtlich kontextualisiert und diskutiert werden. ${ }^{8}$ Das Angebot weitet sich dabei Schritt für Schritt: So werden inzwischen verstärkt Quellen der neueren und neuesten Zeit im WWW publiziert. Und aufgrund der inhaltlichen und methodischen Öffnung der Geschichtswissenschaft in den letzten Jahrzehnten findet ein breites Spektrum von Quellen Berücksichtigung, vom ,gesellschaftlichen Alltag“ bis zur ,hohen Politik“.

Wichtiger Motor für die Publikation von Quellen im Netz sind auch andere Akteure der weit ausdifferenzierten deutschen Geschichtslandschaft. Zahlreiche Gedenkstätten, Museen, Dokumentationsstellen und historische Initiativen suchen die „virtuelle Öffentlichkeit“ und verwenden Quellen für die institutionelle Selbstdarstellung, die Werbung und Bindung von Besuchern oder eine vertiefende zielgruppenorientierte pädagogische Arbeit. ${ }^{9}$ Auch Stiftungen und Einrichtungen historisch-politischer Bildungsarbeit nutzen das World Wide Web verstärkt, um historische Themen mit „authentischem“ Material nahe zu bringen. Der Föderalismus führt dabei zu einer Vervielfältigung der Angebote - zumal durch landesund regionalgeschichtliche Plattformen, die in vielen (Bundes-)Ländern entstanden sind (vgl. Rettinger; Schrade 2006; Schlögl 2006; Weidner 2010). Historische Websites, die Überblicksdarstellungen, Einführungstexte und Basisinformationen mit exemplarischen Quellen verknüpfen, sind jedoch nicht nur auf „Land und Leute" bezogen, sondern auch auf bestimmte Ereignisse und Erinnerungsorte. Die in der Wissenschaft zum Teil beklagte Fixierung der Öffentlichkeit auf Jahrestage und Jubiläen und die zunehmende „Eventisierung“ von Geschichte (Handro 2009:

\footnotetext{
7 In der Regel handelt es sich um Digitalisate (also digitale „Faksimiles“), mitunter um Transkriptionen, z.T. um wissenschaftlich kommentierte Editionen. Vgl. die weiterführenden Hinweise, Erläuterungen und Links unter http://www.historicum.net/recherche/digitalisierte-quellen/textressourcen; http://www.clio-online.de/site/lang_de/95/default.aspx; http://www.ub.uni-heidelberg.de/helios/digi/digiallg.html; http://old.hki.uni-koeln.de/retrodig/ (Übersicht über RetrodigitalisierungsProjekte mit Unterstützung der DFG); http://gdz.sub.uni-goettingen.de/ (Göttinger Digitalisierungszentrum); http://www.digitale-sammlungen.de/ (Münchner Digitalisierungszentrum); http://www.zvdd.de/ (,Zentrales Verzeichnis Digitalisierter Drucke") sowie http://www.intelligent-information.de/hintergrundsinformation/digitalisierungsprojekte-bestandsaufnahme/.

${ }^{8}$ Vgl. die Hinweise in Anm. 26. Zu regional- und landesgeschichtlichen Portalen vgl. Anm. 29.

${ }^{9}$ Dabei gibt es jedoch sehr unterschiedliche Ansätze. Während sich ein großer Teil der Einrichtungen zurückhaltend gegenüber ausgedehnten Web-Präsentionen gibt und das Internet eher selektiv nutzt, haben sich Einrichtungen wie das Deutsche Historische Museum in Berlin oder das Haus der Geschichte in Bonn schon frühzeitig um ihren ,virtuellen“ Auftritt bemüht, Sammlungsdatenbanken zugänglich gemacht oder Ausstellungen im Netz ,archiviert“. Die Beteiligung am Web 2.0 und die offensive Nutzung von Plattformen wie „Flickr“, „Facebook“ oder „YouTube“ für die Öffentlichkeits- und Bildungsarbeit - wie sie etwa vom Anne Frank Haus, Yad Vashem oder dem US Holocaust Memorial Museum praktiziert wird - ist noch nicht allgemein üblich. Vgl. die Beiträge der im April 2011 durchgeführten Tagung „Digital Memory on the Net“ unter http://www.bpb.de/veranstaltungen/EB66Q1.
} 
79ff.) hat zuletzt mehrere Projekte begünstigt, in denen Symbolorte und Wendepunkte deutscher Geschichte multimedial präsentiert werden. ${ }^{10}$

Daneben versuchen auch private Unternehmen, den Bedarf an historischer Rückschau und Selbstvergewisserung, Appeal und Aura historischer Quellen zu nutzen. Die notorischen Digitalisierungsprojekte von Google ${ }^{11}$ haben nicht nur wichtige Buchbestände und Kunstobjekte ${ }^{12}$ „übers Netz“ zugänglich gemacht, sondern den Druck auf öffentliche Einrichtungen erhöht. Projekte wie die „Deutsche Digitale Bibliothek“ oder die digitale Bibliothek „Europeana“13 sind demnach auch als Gegenentwurf zum „Google Books Library Project“ zu verstehen - als Versuch, der vermeintlich drohenden „Amerikanisierung“ des digitalen Gedächtnisses $\mathrm{zu}$ begegnen, ${ }^{14}$ kommerzielle Interessen $\mathrm{zu}$ begrenzen und dem europäischen Kulturgut eine eigene Plattform zu verschaffen (vgl. Euler 2011: 345ff.). ${ }^{15}$ Google ist jedoch nicht der einzige privatwirtschaftliche Akteur im „Digitali10 Vgl. die Hinweise in Abschnitt 5.
11 Seit 2004 digitalisiert Google in Kooperation mit Bibliotheken und Verlagen Buchbestände und
macht die Digitalisate online verfügbar (derzt. unter dem Namen „Google Bücher/books.google“);
dabei wurden neben älteren urheberrechtsfreien Büchern und ,verwaisten Werken“, wo
Rechteinhaber unauffindbar oder unbekant waren, auch Bücher erfasst, für die noch
Urheberrechtsschutz bestand - solange die Rechteinhaber nicht widersprachen. Nach Klagen von
Betroffenen und einem 2008 entwickelten Vergleich zwischen Google und amerikanischen Verlagen
und Autoren, der dem Konzern eine Digitalisierung urheberrechtsgeschützter Bücher gegen
Entschädigung und gewisse Einschränkungen erlaubte, ist die Digitalisierungspraxis seit einem
amerikanischen Urteil vom März 2011 zunächst in Frage gestellt. - Deutsche Buchbestände sind von
Google bisher nur vergleichsweise zurückhaltend erfasst worden, wegen des enger gefassten
deutschen Urheberrechts und größerer Skepsis der dortigen Bibliotheken und Verlage (vgl. Euler
2011).

${ }^{12}$ Vgl. http://www.googleartproject.com/. Das Projekt - laut Spiegel ein „StreetView für Museen“ präsentiert derzeit virtuelle Rundgänge durch siebzehn weltbekannte Museen (darunter das Museum of Modern Art, die Eremitage, die Reina Sofia oder die Alte Nationalgalerie in Berlin) und jeweils hochauflösende Scans von einzelnen Werken der Malerei.

13 Vgl. http://www.deutsche-digitale-bibliothek.de/ und http://www.europeana.eu/portal/. Die 2009 initiierte Deutsche Digitale Bibliothek und die 2007 ins Leben gerufene Europeana sind als Portale mit umfangreichen Suchfunktionen konzipiert, über die man Zugriff auf die Daten und digitalisierten Bestände der beteiligten Bibliotheken und Archive bekommt. Die DDB, die noch in der Konzeptionsphase ist, wird vor allem von großen staatlichen Archiven, Bibliotheken und Wissenschaftsinstituten getragen; an der Europeana oder Europäischen Digitalen Bibliothek wirken derzeit Einrichtungen aus 30 Ländern (vor allem Frankreichs, Spaniens, Italiens, Griechenlands, Deutschlands, Österreichs und des Beneluxraums) mit. Sowohl DDB als auch Europeana wollen nicht nur Bücher und Druckwerke in den Mittelpunkt stellen, sondern Fotosammlungen, Filme und Abbildungen von Objekten erfassen.

14 Befürchtet wird zum einen, dass die von Google betriebene Digitalisierung schwerpunktmäßig amerikanisches Kulturgut erfasst, zum anderen, dass die digitalisierten europäischen Quellen aus rechtlichen Gründen nur amerikanischen Nutzern zugänglich sein könnten.

15 Bisher bleibt die Europeana jedoch deutlich hinter Google zurück. Aus urheberrechtlichen Gründen, aber auch wegen des geringeren finanziellen Einsatzes der öffentlichen Hand und institutioneller Blockaden verfügt die Europeana bisher über deutlich weniger Digitalisate als die Google-Datenbank. Deutsche Quellen sind bislang vergleichsweise spärlich vertreten. Vgl. die Angaben unter http://www.spiegel.de/netzwelt/web/0,1518,591570,00.html; http://www.welt.de/kultur/article5004877/Literatur-kann-man-gut-ohne-Google-digitalisieren.html und http://www.spiegel.de/netzwelt/netzpolitik/0,1518,753229,00.html. 
sierungsgeschäft“. Auch andere Anbieter engagieren sich auf dem Markt für historische Stoffe und Dienstleistungen, wie vor allem der Spiegel-Konzern ${ }^{16}$ oder der Zenodot-Verlag mit seiner Volltextbibliothek ,zeno.org ““. ${ }^{17}$

Schließlich sind Initiativen der Nutzer selbst zu nennen. So kursieren vielfach Quellentexte, gescannte Fotografien oder Filmausschnitte - teilweise unter Missachtung von Urheber- oder Archivrechten - in den verstreuten Foren zu historischen Themen oder Portalen wie „Flickr“ oder „YouTube“. Es gibt aber auch Bemühungen um eine koordiniertere Zusammenstellung historischer Quellen, in erster Linie natürlich bei dem 2001 gegründeten deutschen „Wikipedia“ - wo man den Status des „Amateurhaften“ zunehmend abzustreifen versucht, ${ }^{18}$ mit dem „Schwesterprojekt“ (Cyron 2009: 262) „Wikisource“ seit 2005 eine Sammlung rechts-, politik- und kulturgeschichtlicher Quellen entwickelt und dabei mittlerweile auf professionelle Unterstützer wie das Bildarchiv des Bundesarchivs und die Deutsche Fotothek zurückgreifen kann (Beine 2009; Sahle 2009). ${ }^{19}$

Das Internet etabliert sich so immer mehr als wichtiges „Gedächtnismedium“ (Assmann 2006: 243), als Struktur, die historische Quellen direkt zugänglich macht. Dennoch gibt es auch gegenläufige Tendenzen. Kommerzielle Interessen, aber auch berechtigte urheber-, archiv- und datenschutzrechtliche Vorbehalte (vgl. Burckhardt; Hohls; Ziegeldorf 2005: 49ff.; Euler 2011) stehen vor allem bei

\footnotetext{
16 Der Spiegel-Verlag stellt nicht nur die früheren Ausgaben des Nachrichtenmagazins online zur Verfügung, sondern betreibt das quellenorientierte Geschichtsportal „einestages“ und ist führender Akteur auf dem Markt für Geschichtsdokumentationen und „Histotainment“ (Spiegel TV). Auch das ursprünglich eigenständige Projekt „Gutenberg-De“, das sich die Webpräsentation urheberechtsfreier (literarischer) Texte vorgenommen hatte, ist mittlerweile vom Spiegel-Verlag aufgegriffen und an Spiegel Online angedockt worden; vgl. http://gutenberg.spiegel.de/. Andere Medienunternehmen wie der ZEIT-Verlag oder Gruner + Jahr agieren ebenfalls auf dem Markt für historische Inhalte und halten Online-Angebote mit redaktionellen Inhalten, die auch vereinzelt Quellen vorstellen. Vgl. etwa http://www.zeit.de/wissen/geschichte/index oder http://www.geo.de/GEO/heftreihen/geo_epoche/magazinuebersicht.html.

17 Vgl. http://www.zeno.org/.

18 Das zeigt sich u.a. daran, dass sich Wikipedia um Redaktionen für bestimmte Themenbereiche bemüht, Qualitätssiegel für fundierte Artikel vergibt, die Veränderbarkeit ausgereifter Beiträge einschränkt, Zugriffsrechte abstuft, Propagandaattacken und „Edit wars“ zu unterbinden versucht. Auf der anderen Seite versuchen Einrichtungen politisch-historischer Bildungsarbeit zunehmend auf die Wikipedia-Artikel Einfluss zu nehmen; so möchte etwa ein Projekt der Friedrich-Ebert-Stiftung die Beiträge zur Geschichte der Arbeiterbewegung auf den „richtigen“ Stand bringen (vgl. http://www.fes.de/hfz/forschung/inhalt/projekte.htm\#projekt_woyke). Zur Kritik der „Professionalisierungsbestrebungen“ bei Wikipedia vgl. Cyron 2009; Lorenz 2009a: 296ff., 305f. bzw. Lorenz 2009b; Meyer 2009a: 279f.; Pscheida 2010: $331 \mathrm{ff}$.

${ }^{19}$ Vgl. http://de.wikisource.org. Veröffentlicht werden Quellen, die urheberrechtsfrei sind oder unter einer freien Lizenz stehen. Das Bundesarchiv hat 2008 ca. 100.000 Fotos aus seinen Beständen für die Nutzung über Wikisource freigegeben, so lange Archiv und Urheber genannt und die Dateien nur zu gleichen Bedingungen weitergegeben werden (Creative-Commons-Lizenz BY-SA). Seit 2009 gibt es eine Zusammenarbeit mit der Deutschen Fotothek der Sächsischen Landesbibliothek - Staats- und Universitätsbibliothek Dresden, die Wikisource zunächst 250.000 Bilder zur Verfügung stellen will.
} 
zeithistorischen und massenmedialen Quellen einem schnellen Zugriff entgegen. ${ }^{20}$ Oft benutzen Museen und Gedenkstätten Quellen, zumal Bilder, nur als „Teaser“ oder Orientierungspunkte, da das Internet Interesse wecken, aber nicht den Ausstellungsbesuch ersetzen und bei sensiblen historischen Themen eine visuelle „Reizüberflutung“ vermieden werden soll (vgl. Hein 2009a: 159ff., 212ff., 254, 257; Hein 2009b: 161 ff.). Abgesehen von grundsätzlicheren Einwänden gegen die Digitalisierung verfügen viele Archive, Forschungseinrichtungen und Museen nicht über die personellen, finanziellen und technischen Möglichkeiten für eine rasche und systematische Onlinestellung ihrer Bestände. So werden historische Quellen meist nur exemplarisch und projektorientiert zugänglich gemacht. Dies gilt noch mehr für die Privatinitiativen von Internetnutzern, deren Quellenerschließung oft punktuell, impressionistisch und persönlich motiviert ist. Das Internet ist also kein eigenständiges, umfassendes Archiv, sondern eine selektive „Benutzeroberfläche“ für die historische Überlieferung.

\section{Quellensuche}

Macht man sich im Internet nun auf die Suche nach historischen Quellen zur deutschen Geschichte ${ }^{21}$ oder brauchbaren Materialien für eine moderne, historisch fundierte Landeskunde ${ }^{22}$, so wird man mit der üblichen Suche per Google (Yahoo u.a.) schon recht weit kommen. ${ }^{23}$ Wer dort einschlägige Begriffe wie „Ermächti-

\footnotetext{
20 So sind viele digitalisierte Quellen, die urheberrechtlichen Beschränkungen unterliegen oder kommerziell vermarktet werden (v.a. Fotos und Filme), nur nach Registrierung und gegen Entgelt recherchier- und nutzbar (also Teil des „Hidden Web/Invisible Web“).

${ }^{21}$ Im Folgenden kann die „Quellenlage“ im World Wide Web nur oberflächlich und ausschnitthaft dargestellt werden. Genauere Ausführungen zu Struktur, Aufmachung, inhaltlicher Qualität und didaktischer Brauchbarkeit der genannten Websites müssen aus Platzgründen entfallen. Hier können nicht nur kommentierte Linklisten weiterhelfen, sondern vor allem die Web-Rezensionen und Internettipps, die im Portal H-Soz-u-Kult sowie seit der Jahrtausendwende regelmäßig in den Zeitschriften „Geschichte in Wissenschaft und Unterricht", „Geschichte Lernen“ und „Praxis Geschichte" erscheinen, vgl. http://hsozkult.geschichte.hu-berlin.de/rezensionen/type=rezwww und http://www.praxisgeschichte.de/unterricht/index_links.php.

22 „Modern“ zielt hier auf den Ansatz einer neueren, nicht nur Fakten vermittelnden, sondern kulturwissenschaftlich grundierten, handlungsorientierten, kommunikativen und interkulturell ausgerichteten Landeskunde (vgl. die verschiedenen Beiträge in Krumm et al. 2010: 1378-1511 bzw. die Artikel von Lüsebrink, Leupold und Krumm in Bausch; Christ; Krumm ${ }^{4} 2003$ : 60ff., $127 \mathrm{ff} ., 138 \mathrm{ff}$. sowie Huneke; Steinig 52010: 84ff.; Storch 2001: 285ff.). „Modern“ soll hier aber auch zeitlich verstanden werden: Wenn Landeskunde - wie die kanonische Formulierung der ABCD-Thesen lautet - „, in hohem Maße [...] Geschichte im Gegenwärtigen“ ist, so können sicher auch historische Themen wie die Reformation und das Zeitalter der Glaubenskriege oder Absolutismus und Aufklärung landeskundlich von Interesse sein, im Hinblick auf die historischen Grundlagen der Gegenwart kommt jedoch schwerpunktmäßig die Geschichte des 19-21. Jahrhunderts in Betracht.

${ }^{23}$ Einführende Hinweise für das historische Arbeiten mit Hilfe des Internet oder des World Wide Web bieten z.B. Eder et al. 2006; Gasteiner; Haber 2010; Grosch 2002; Jenks; Marra 2001; die Suche nach historischen Quellen steht dabei jedoch nicht (immer) im Mittelpunkt. Grundlegend zu medienadäquaten Recherchestrategien auch Haber 2009: 66ff., $162 \mathrm{ff}$.
} 
gungsgesetz“, „Thesenanschlag“, „Faust“, „Kommunistisches Manifest“ oder „Novemberrevolution“ eingibt, erhält nicht nur Seiten mit einführenden Informationen, sondern auch direkt Zugriff auf zentrale Dokumente und Bildquellen. Das hat vor allem mit der unaufhaltsamen Ausweitung des „Wikipedia-Universums" (Pscheida 2010) und den Suchalgorithmen von Google u.a. zu tun. ${ }^{24}$ Ein Grund hierfür ist aber auch, dass öffentliche Bildungseinrichtungen Strategien entwickeln, um dafür zu sorgen, dass ihre Seiten unter den ersten Fundstellen sind und nicht in einer Treffermenge von 368.000 untergehen - und dabei zum Teil sogar die Zusammenarbeit mit Suchmaschinenbetreibern suchen. ${ }^{25}$ Dennoch ist „googeln“ nicht alles. Abgesehen davon, dass man die Trefferlisten der Suchmaschinen kritisch reflektieren sollte (vgl. auch grundlegend Haber 2005: 86f.), können deren Ergebnisse bei komplexeren Themen immer noch intransparent und unübersichtlich sein. Je weiter man sich von den großen historischen Ereignissen und ikonischen Figuren der Geschichte entfernt, sach-, begriffsbezogen oder zeitübergreifend sucht, desto eher muss man auch andere Wege ausprobieren.

Da übergreifende Quellenportale wie Wikisource oder Europeana derzeit noch über ein recht lückenhaftes Angebot verfügen, bietet sich an, auch auf Empfehlungen und Linklisten von Fachportalen zurückzugreifen. Zunächst zu nennen sind hier die aus wissenschaftlicher Sicht zentralen Plattformen „Clio online“, „historicum.net“ oder „Zeitgeschichte-online“"26, aber auch geschichtsdidaktische Angebote wie auf „Lehrer-Online“. ${ }^{27}$ Sie erschließen die Weiten des World Wide

24 Vgl. zur Bedeutung der Suchmaschinen Meyer 2009c: 180ff.; grundlegend Lehmann; Schetsche 2005; Machill; Beiler 2007; Wiedmaier 2007. Kritik findet immer wieder, dass die Suchalgorithmen der Anbieter nicht offen liegen, das Ranking nicht transparent ist und die Trefferlisten von inhaltlichen Vorentscheidungen und kommerziellen Interessen geprägt sind. Zur Bevorzugung Wikipedias durch Google vgl. Lorenz 2009b: $217 \mathrm{f}$.

25 Vgl. Meyer 2009a: 277 sowie die Beiträge der Tagung „Digital Memory on the Net“ von 2011; http://www.bpb.de/veranstaltungen/EB66Q1.

${ }^{26}$ Bei Clio online findet man unter dem Menüpunkt „Web-Verzeichnis“ (http://www.clio-online.de/site/lang_de/40208087/default.aspx) zahlreiche Unterverzeichnisse mit Hinweisen auf WWWRessourcen, für die Quellensuche besonders hilfreich: „Materialien“, „Multimedia“ und „Quellen“. „historicum.net“ (http://www.historicum.net/home/) bietet Themen- und Ländermodule, die jeweils in einem Unterpunkt wichtige Quellen vorstellen und dabei ausgewählte Links bieten; erwähnenswert auch der Bereich „Lehren \& Lernen“ (http://www.historicum.net/lehren-lernen/), der einführende Hinweise zum Thema Geschichte und Internet gibt, sowie das zu historicum.net gehörende E-Journal Zeitenblicke (http://www.zeitenblicke.de/). - „Zeitgeschichte-online“ (ZOL) bietet neben Aufsätzen, Vortragsvideos, Ausstellungsrezensionen oder Filmbesprechungen umfassendere Themenmodule mit Hinweisen auf Online-Ressourcen und ein Verzeichnis zu „Texte \& Quellen“, das kommentierte Links zur Verfügung stellt (vgl. http://www.zeitgeschichteonline.de/go/rainbow/95/de/DesktopDefault.aspx) - allerdings weitgehend übereinstimmend mit Clio online, dem ZOL zugeordnet ist. Unter Zeitgeschichte-online wird auch auf die Fachzeitschrift „Zeithistorische Forschungen“ verlinkt (vgl. http://www.zeithistorische-forschungen.de/site/40208106/defaultaspx), deren Themenhefte regelmäßig interessante Beiträge zu wichtigen Quellen und Quelleneditionen liefern. - Wichtige Informationsangebote (im Aufbau) sind auch http://www.ieg-ego.eu/ (European History Online) und http://docupedia.de/zg/Hauptseite (Docupedia-Zeitgeschichte).

${ }^{27}$ Vgl. bei Lehrer-Online insbesondere das Verzeichnis „Unterricht“, das für den Bereich Geschichte hilfreiche Unterrichtseinheiten und Fachmedien vorstellt, Didaktisierungsmöglichkeiten thematisiert 
Web thematisch, verweisen auf wichtige WWW-Quellen und multimediale Angebote, ermöglichen mit Hilfe eigener Suchfunktionen eine beschleunigte Annäherung an Inhalte wie Materialien und liefern teilweise hilfreiche redaktionelle Einschätzungen. Portale wie „Virtual Library Museen“, das „Online Gedenkstättenforum“ oder das „BAM-Portal“ bieten Informationen und Linklisten für den Rechercheeinstieg und die weitere Erkundung der Museums- und Gedenkstättenlandschaft; zum Teil ermöglichen sie auch einen direkten Zugriff auf digitalisiertes Sammlungsgut und themenbezogene Internetpräsentationen. ${ }^{28}$ Wichtige Ansatzpunkte für die Recherche sind auch die Einrichtungen politischhistorischer Bildung. Sie sind in jüngster Zeit zu wichtigen Akteuren ,digitaler Geschichtsvermittlung“ geworden und bieten zum Teil zeitlich und thematisch breit angelegte Internetdossiers mit Fachartikeln, Glossar, Literaturschau, illustrierenden Quellen oder weiterführenden Links. ${ }^{29}$

und kommentierte Linksammlungen zu historischen Quellen und anderen Materialien bietet. Brauchbar auch http://www.geschichte-lernen.de/go/Hilfreiche+Links (thematisch geordnete, kommentierte Links). Das Portal „Lernen aus der Geschichte“ bündelt Materialien und Ressourcen zum Thema NS-Geschichte und gibt dabei zahlreiche Hinweise auf andere Internetpräsentationen und WWW-Quellen; im Verzeichnis „Lernen \& Lehren“ finden sich thematische Dossiers, die jeweils auch externe Links kommentieren und einbinden, vgl. http://lernen-aus-der-geschichte.de/Lernenund-Lehren.

28 Vgl. http://www.historisches-centrum.de/index.php?id=272 (Virtual Library Museen, allerdings veraltet); http://www.gedenkstaettenforum.de/ (bietet auch Links zu einzelnen Projekten oder privaten Initiativen); http://www.bam-portal.de/ (Portal für Bibliotheken, Archive, Museen, ermöglicht objektorientierte Suche). Hilfreich auch http://www.dhm.de/links.html (Linkadressen von Museumsverbänden und -portalen); http://www.museumsbund.de/de/links/national/ bzw. http://www.museumsbund.de/fileadmin/geschaefts/dokumente/Wir_Mitgliedschaft/Museums-

verzeichnis_2009.pdf; http://www.clio-online.de/site/lang_de/54/Default.aspx (Museenübersicht bei Clio online); http://www.gedenkstaetten-uebersicht.de/WebObjects/ITF.woa/wa/europa (mit länderbezogenem Zugriff); http://www.ns-gedenkstaetten.de/ (Portal der NRW-Gedenkstätten mit Einstieg zu Gedenkstätten anderer Bundesländer).

${ }^{29}$ Hinter der Bundeszentrale für politische Bildung (http://www.bpb.de/), die auf diesem Gebiet eine besonders extensive Tätigkeit entfaltet (historische Dossiers mit ergänzenden Quellen und Verweisen auf WWW-Ressourcen finden sich im Verzeichnis „Themen \Geschichte“, allgemeine Linktipps im Verzeichnis „Wissen“), stehen die Landeszentralen etwas zurück; sie konzentrieren sich stärker auf Projektförderung, Schriftenvertrieb und Veranstaltungsmanagement und haben meist reduzierte Internetangebote. Vgl. jedoch http://www.geschichte.nrw.de/; http://www.lpbbw.de/geschichtsdossiers.html; http://www.infoseiten.slpb.de/ sowie das gemeinsame Portal von Bundes- und Landeszentralen http://www.politische-bildung.de/. - Ausführliche Internetpräsentationen zur deutschen Geschichte bieten die landesgeschichtlichen Portale, die von Bibliotheken, Landschaftsverbänden, landesgeschichtlichen Ämtern oder Instituten getragen werden. Quellen werden hier einmal illustrativ in Artikel eingebunden, zum anderen aber auch in gesonderten Modulen vorgestellt und erläutert. Vgl. etwa http://www.bayerische-landesbibliothek-online.de/; http://lagis.online.uni-marburg.de/de/(Hessen); http://www.lwl.org/westfaelische-geschichte/portal/Internet/haupt.php?urlNeu= und http://www.lwl.org/LWL/Kultur/Aufbruch/start_html/seite2/start2_html; http://www.rheinische-geschichte.lvr.de/Seiten/home.aspx (im Aufbau); http://www.vimu.info/general_01.jsp (Schleswig-Holstein); http://www.landesarchiv-bw.de) web/50999 (Baden-Württemberg, in der Konzeptionsphase); http://www.sachsendigital.de/; http://www.ooegeschichte.at/Wir-Oberoesterreicher.1294.0.html sowie als Überblick Rettinger; Schrade 2006; Schlögl 2006; Weidner 2010. 
Auch der öffentlich-rechtliche Rundfunk ist ein wichtiger Lieferant für historische Materialien und Informationen. Mit dem Boom des Geschichtsfernsehens und der Profilierung eines bildungsorientierten Kulturradios sind in den letzten zehn Jahren etliche sendebegleitende Internetangebote entstanden, die weiterführende Informationen zur Verfügung stellen und sich vereinzelt sogar zu virtuellen Ausstellungen entwickelt haben. ${ }^{30}$

Die Archive und Bibliotheken als eigentliche „Hüter der Überlieferung“ und Lieferanten der Quellen treten demgegenüber etwas zurück. Sie tragen das größte Gewicht bei der Erschließung und Digitalisierung, setzten aber mit Blick auf die Sicherung von Kulturgut und die akademische Forschung zunächst andere Schwerpunkte als die Hauptakteure historisch-politischer Bildungsarbeit und populärer Geschichtsvermittlung. Ihr Augenmerk galt weniger der Präsentation von Schlüsseldokumenten und bildhaften, „sprechenden“ Quellen als vielmehr der Aufbereitung größerer Quellenkorpora, die ideen-, kultur- und wissenschaftsgeschichtlich von Interesse, hinsichtlich Inhalt, sprachlicher Komplexität, Struktur und Anschaulichkeit allerdings nicht für die didaktische Arbeit (geschweige denn den Fremdsprachenunterricht) geeignet sind.

Allerdings gibt es inzwischen auch bei den Archiven eine verstärkte Wendung zum Publikum (vgl. Jakobi 2000; Kruse 2008: 30f., 44f.; Lersch; Müller 2010; Schmitt 2010; Storm 2010), einmal mit Archivportalen, die über Leistungen und Bestände der überregionalen, regionalen und lokalen „Gedächtnisorte“ informieren, ${ }^{31}$ zum zweiten mit archivpädagogischen Modulen, die Dossiers für die Bildungsarbeit, Studium und Schule bereitstellen. ${ }^{32}$

\footnotetext{
${ }^{30}$ Die Zahl der hierbei entstandenen, oft qualitativ guten, zumal für die Bildungsarbeit geeigneten Angebote war groß, ist nach dem 12. Rundfunkänderungsstaatsvertrag (vgl. Anm. 68) aber stark zurückgegangen. Wichtige historische Internetausstellungen des öffentlich-rechtlichen Rundfunks gibt es derzeit (Juli 2011) beispielsweise noch zur preußischen Geschichte (http://www.preussenchronik.de/), zur Geschichte der deutsch-polnischen Beziehungen (http://www.deutsche-undpolen.de/) oder zur Geschichte des Auschwitz-Prozesses (http://www.hr-online.de/website/static/spezial/auschwitzprozess/index.html). Besonders breit gefächert sind die Serviceangebote zur Geschichte der DDR und der deutschen Wiedervereinigung (vgl. Anm. 99).

31 Ein bundesweites Archivportal gibt es noch nicht, jedoch haben fast alle deutschen Länder entsprechende Angebote eingerichtet; als Beispiel http://www.archive.nrw.de/; Übersicht unter http://www.archivschule.de/service/archive-im-internet/archive-in-deutschland/archivportale/regionale-archivportale-im-internet.html.

32 Vgl. die Angebote unter http://www.bundesarchiv.de/oeffentlichkeitsarbeit/bilder_dokumente/index.html.de; http://www.digam.net/ (Digitales Archiv Marburg); http://www.digada.de/ (Digitales Archiv Hessen-Darmstadt). Mittlerweile haben die meisten Archive pädagogische Angebote (vgl. http://www.archivpaedagogen.de/), während umfangreichere Online-Module noch die Ausnahme darstellen.
} 


\section{Quellensorten und -gruppen}

Versucht man aufgrund eines ersten Überblicks die „Quellenlage im Internet“ nach Quellensorten oder -gruppen aufzuschlüsseln, so ergibt sich folgendes Bild.

- Wer den rechtlichen und politischen Rahmen der deutschen Geschichte erarbeiten möchte, kann über rechtsgeschichtliche Websites, Auftritte von Ministerien und anderen politischen Einrichtungen sowie Bibliotheks- und Archivseiten neben den Verfassungen als Schlüsseltexten deutscher Staatlichkeit und „klassischer" Staatsbürgerkunde auch auf das Reichs- und Bundesgesetzblatt sowie die wesentlichen Gesetzbücher zurückgreifen. ${ }^{33}$ So lässt sich die rechtliche Entwicklung des 19.-20. Jahrhunderts für den Bereich des Deutschen Reiches (aber ebenso für Österreich und die Schweiz ${ }^{34}$ ) in Vielem nachzeichnen. Auch für einzelne Länder wie Preußen und Bayern und frühere Epochen liegen digitalisierte Rechtstexte vor. ${ }^{35}$ Zugänglich sind überdies wichtige parlamentarische Quellen wie die Protokolle des Deutschen Reichstags von 1867-1942 und die Protokolle des Deutschen Bundestages; 36 für die Länderparlamente gibt es - zumeist für die jüngere Zeit - ebenfalls zahlreiche Digitalisate oder Transkripte. ${ }^{37}$ Hinzu kommen Dokumente der Exekutive (etwa in Gestalt der Akten der Reichskanzlei in der

\footnotetext{
33 Vgl. http://www.documentarchiv.de/ (19-21. Jahrhundert); http://www.verfassungen.de/; die zentralen Verfassungs- und Rechtstexte sind auch zu finden unter: http://www.dhm.de/lemo/home.html; http://www.bpb.de/wissen/VGB5GU,0,0,Gesetze.html oder http://de.wikisource.org/wiki/Hauptseite zu finden. Für das Reichsgesetzblatt vgl. http://alex.onb.ac.at/gesetze_drab_fs.htm, für das Bundesgesetzblatt http://www.bgbl.de/index.php. - Das deutsche Strafgesetzbuch findet sich z.B. unter http://de.wikisource.org/wiki/Strafgesetzbuch_f $\%$ C3\%BCr_das_Deutsche_Reich_(1871), die neueste Fassung unter http://www.gesetze-im-internet.de/bundesrecht/stgb/gesamt.pdf, das Bürgerliche Gesetzbuch von 1896 mit seinen Änderungen unter http://lexetius.com/BGB/Inhalt, die jüngste Fassung unter http://www.gesetze-im-internet.de/bundesrecht/bgb/gesamt.pdf.

34 Vgl. für Österreich v.a. http://alex.onb.ac.at/alex.htm (Gesetzesblätter bis 1945) und http://www.ris.bka.gv.at/default.aspx (nach 1945); für die Schweiz http://www.amtsdruckschriften.bar.admin.ch/showHome.do (Bundesblatt, Diplomatische Dokumente, Bundesratsprotokolle); http://db.dodis.ch/dodis/dodis?_l=de (Diplomatische Dokumente der Schweiz); http://www.ssrq-sds-fds.ch/online/ (Sammlung Schweizerischer Rechtsquellen).

${ }^{35} \mathrm{Vgl}$. als herausragende Beispiele die Interneteditionen zum Sachsenspiegel und zum Westfälischen Frieden; http://www.sachsenspiegel-online.de/cms/ und http://www.pax-westphalica.de/ipmipo/index.html. Für den landeskundlichen $\mathrm{DaF}-$ Unterricht sind derartige Texte aber natürlich schon sprachlich nicht geeignet.

36 Vgl. http://www.reichstagsprotokolle.de/index.html sowie http://dip.bundestag.de/ (Bundestagsprotokolle, derzeit ab 1976). - Einführende Texte zur Parlamentarismusgeschichte mit begleitenden Quellentexten und Bildern: http://www.bundestag.de/kulturundgeschichte/geschichte/parlamentarismus/index.jsp. Dort werden auch Videos aus der Volkskammer der DDR zur Verfügung gestellt: http://www.bundestag.de/kulturundgeschichte/geschichte/parlamentarismus/10_volkskammer/mediathek/index.jsp.

37 Vgl. nur die Einstiegsseite http://www.parlamentsspiegel.de/ps/inhalt/links-parlamentsdokumentation.jsp; historisch weiter zurückreichend: http://www.bayerische-landesbibliothek-online.de/landtag-digital.
} 
Weimarer Republik oder der Kabinettsprotokolle der Bundesregierung ${ }^{38}$ ) sowie wichtige bilaterale oder internationale Verträge. ${ }^{39}$

- Für eine inhaltlich und visuell verdichtete, sozial- oder kulturgeschichtliche Deutung deutscher Politik sind jedoch andere Quellen und Ausdrucksformen erforderlich: Druckschriften, Parteiprogramme, Flugblätter, Plakate, Karikaturen. ${ }^{40}$ Neben wenigen Quellen zu Reformation, Bauernkrieg und den konfessionellen Konflikten der Frühen Neuzeit ${ }^{41}$ und digitalisierten Sammlungen zur Revolution von 1848/4942 liegt vor allem für das 20. Jahrhundert wichtiges Material vor. Natürlich liefert das Netz keine lückenlose Dokumentation. Über verschiedene Plattformen - von der Friedrich-Ebert- und Konrad-Adenauer-Stiftung über das Deutsche Historische Museum und das Bundesarchiv bis zur Bundeszentrale für politische Bildung - lässt sich jedoch eine aufschlussreiche Reihe von politischen Parolen, Statements, Wahlplakaten und Bildpostkarten erstellen. ${ }^{43}$ Die im Netz

38 Vgl. http://www.bundesarchiv.de/aktenreichskanzlei/1919-1933/0000/index.html (Akten der Reichskanzlei) sowie http://www.bundesarchiv.de/cocoon/barch/0000/index.html (Kabinettsprotokolle, derzeit bis in die 1960er Jahre reichend) und die Darstellung von Jörg Filthaut und Uta Rössel in Jahrbuch 2007: $75 \mathrm{ff}$.

39 Vgl. die Angebote der Ministerien, etwa die Darstellungen und Quellen unter http:/ /www.auswaertiges-amt.de/sid_14106E50B2410825DB57BB7FA3706C9D/DE/AAmt/PolitischesArchiv/EinblickeindasArchiv_node.html sowie http://www.staatsvertraege.de/.

40 Die im WWW zur Verfügung gestellten historischen Konversationslexika und Wörterbücher (Zedler, Meyer's, Brockhaus etc.) können zwar wichtige Einblicke in die Entwicklung der politischen Semantik bieten, eignen sich i.d.R. aber auch kaum für die Verwendung im Fremdsprachenunterricht. Vgl. nur http://www.retrobibliothek.de; http://www.zeno.org/Zeno/-/Lexika oder http://www.zedler-lexikon.de/.

41 Vgl. den Überblick unter http://www.historicum.net/themen/bauernkrieg/links/; http://www.historicum.net/themen/reformation/quellen/; http://digbib.bibliothek.uni-augsburg.de/dda/flugschriften_0001.html; http://www.onb.ac.at/onbarchiv/flu/1848/index.htm sowie die Portale für deutschsprachige Drucke des 16. und 17. Jahrhunderts, die allerdings nur zu einem kleineren Teil digitalisiert sind, http://www.bsb-muenchen.de/1681.0.html (VD 16) und http://www.vd17.de/.

42 Vgl. http://edocs.ub.uni-frankfurt.de/1848/1848.htm; http://www.zlb.de/digitalesammlungen/f4index.php?collection=2\&layer $=2 ;$ http: $/ /$ www.onb.ac.at/onbarchiv $/$ flu $/ 1848 /$ index.htm und die ältere Internetpräsentation http://www.zlb.de/projekte/1848/index.html. - Allein die Schrift lässt eine Verwendung solch älterer Flugschriften im DaF-Unterricht schwierig erscheinen.

$43 \mathrm{Vgl}$. www.dhm.de/sammlungen/plakate/bestand.html (Beispiele aus der Plakatsammlung des Deutschen Historischen Museums); http://www.bild.bundesarchiv.de/collections/2265753/_1311598214/? (Plakatsammlung des Bundesarchivs); http://plakatarchivaustria.onb.ac.at/; http://www.museum-folkwang.de/de/sammlung/deutsches-plakat-museum.html (stellt wenige „Highlights“ vor); http://www.bildpostkarten.uni-osnabrueck.de/index.php. - Interessante (wenngleich hinsichtlich der Abbildungsqualität nicht immer befriedigende) thematische Sammlungen finden sich beispielsweise unter: http://digitalgallery.nypl.org/nypldigital/explore/dgexplore.cfm?topic=history\&col_id=211 (,World War I Photograph Albums and Postcards“); http://www.ww1-propaganda-cards.com (Erster Weltkrieg); http://content.lib.washington.edu/postersweb/ („War Posters Collection“, Erster und Zweiter Weltkrieg); http://www.calvin.edu/academic/cas/gpa/ („German Propaganda Archive", zu NS-Regime und DDR); http://ddr-plakate.de/; http://www.dhm.de/ausstellungen/kkv/ (Kunst! Kommerz! Visionen! Deutsche Plakate 1888-1933“); http://www.dhm.de/ausstellungen/grundrechte/ („Die Grundrechte im Spiegel des Plakats 1919 bis 1999"); http://www.bundesarchiv.de/oeffentlichkeitsarbeit/bilder_dokumente/00659/index.html.de („Öffentlich angeschlagen - Politische Plakate"); http://www.fes.de/archiv/adsd_neu/inhalt/downloads/weimar_plakat.htm („Politische Plakate der Weimarer Republik“); 
greifbaren Plakatsammlungen gewähren zudem Einblick in andere Felder - etwa der Wirtschafts-, Konsum- ${ }^{44}$ Kultur- oder Mediengeschichte.

- Auch Zeitungen und Zeitschriften sind in den letzten Jahren verstärkt digitalisiert oder über Transkripte im Netz zugänglich gemacht worden. Darunter ${ }^{45}$ finden sich satirische Blätter des 19. und frühen 20. Jahrhunderts wie der Kladderadatsch oder der Simplicissimus, Karl Kraus' Fackel, ${ }^{46}$ Publikationsorgane sozialdemokratischer und sozialistischer Gruppierungen sowie die Exilpresse der NS-Zeit. ${ }^{47}$ In landeskundlicher Hinsicht noch interessanter sind die Angebote der bundesrepublikanischen Leitmedien „Die Zeit"“48 und „Spiegel“49. Beide Zeitungen haben seit einiger Zeit ein gut recherchierbares Webarchiv, das ohne wesentliche Einschränkungen benutzbar ist und bis in die 1940er Jahre zurückreicht. ${ }^{50}$

http://www1.bpb.de/methodik/31P06X,0,0,Wahlplakate_im_Spiegel_der_Zeit.html;

http://www.hdg.de/karikatur/view/karikaturen.html (,50 Jahre deutsche Frage in Karikaturen“); http://www.dhm.de/ausstellungen/kalter_krieg/aus.htm (,Deutsch-Deutsche Feindbilder in der politischen Propaganda 1945 bis 1963“); http://www.hdg.de/film/class125_id1000564.html („,50 Jahre deutsche Geschichte in Kinoplakaten"); http://www.hdbg.de/karikatur/de/a_home/a_fr.htm („,Bayern \& Preußen. Eine historische Beziehung in Karikaturen“); http://www.kas.de/wf/de/71.5707/ (Plakat- und Filmdatenbank des Archivs für Christlich-Demokratische Politik) bzw. http://www.kas.de/wf/de/71.9048/ (Angebot der Konrad-Adenauer-Stiftung zur Geschichte der CDU); http://www.fes.de/archiv/adsd_neu/inhalt/sammlung/audiovisuell/plakatsammlung.htm (Plakatsammlung der Friedrich-Ebert-Stiftung). Historische Wahlergebnisse sind z.B. unter http://www.wahlen-in-deutschland.de/ zu finden.

${ }^{44}$ Vgl. zur Konsum- und Produktgeschichte auch http://www2.wu-wien.ac.at/werbung/ (Reklamemarkensammlung); http://www.bibliothek.uni-regensburg.de/mmz/hwa.htm (Historisches Werbefunkarchiv mit wenigen Hörbeispielen); http://www.alltagskultur-ddr.de/pages/sam/sam.html. Umfassendere Quellenpräsentationen aus dem Bereich der Wirtschaftsgeschichte liegen bisher kaum vor; einzelne Materialien sind jedoch unter Umständen über die Websites von Industrie-, Technikund Agrarmuseen oder Unternehmensarchiven zu erhalten.

45 Zur Digitalisierung von historischen Zeitungen durch Bibliotheken vgl. z.B. die Überblicke bei http://zefys.staatsbibliothek-berlin.de/list/; http://digipress.digitale-sammlungen.de/de/fontSize1/papers-overview/static.html; für Österreich: http://anno.onb.ac.at/.

46 Vgl. http://www.ub.uni-heidelberg.de/helios/digi/kladderadatsch.html; http://www.simplicissimus.info/; http:// corpus1.aac.ac.at/fackel/ (erfordert Registrierung) bzw. http://de.wikisource.org/wiki/Die_Fackel (Auszüge); http://www.ub.uni-heidelberg.de/helios/digi/ulkhd.html (Ulk - Illustriertes Wochenblatt für Humor und Satire). Zu digitalisierten literarischen Zeitschriften des 18. und 19. Jahrhunderts vgl. http://www.ub.uni-bielefeld.de/diglib/aufklaerung/; eine (allerdings nicht vollständige) Übersicht über digitalisierte Zeitschriften unter http://de.wikisource.org/wiki/Zeitschriften.

47 Vgl. zu den verschiedenen Digitalisierungsprojekten der Friedrich-Ebert-Stiftung http://library.fes.de/inhalt/digital/zeitschriften.htm (u.a. Die Arbeit, Die Naturfreunde, Die neue Zeit, Sozialistische Monatshefte); http://library.fes.de/inhalt/digital/pressedienst.htm (Sozialdemokratischer Pressedienst); für Österreich http://www.arbeiter-zeitung.at/ (erfordert Registrierung). - Digitalisate zur NS-Exilpresse unter http://deposit.ddb.de/online/exil/exil.htm.

48 Vgl. http://www.zeit.de/archiv/index.

${ }^{49}$ Vgl. http://www.wissen.spiegel.de bzw. http://www.spiegel.de/spiegel/print/.

${ }^{50}$ Dies ist umso bemerkenswerter, als andere Zeitungsarchive lückenhaft sind, nur aktuellere Beiträge erfassen bzw. nur gegen Bezahlung zu nutzen sind (so auch bei der Frankfurter Rundschau, der Süddeutschen und der Frankfurter Allgemeinen Zeitung). Derzeit in Teilen öffentlich zugänglich: http://www.berlinonline.de/berliner-zeitung/archiv/.bin/dump.fcgi/2011/index.html (Berliner Zeitung, ab 1994); http://www.freitag.de/archiv (Freitag, ab 2005); http://www.jungewelt.de/suche/ (junge welt, ab 1997); http://www.neues-deutschland.de/suche/ (Neues Deutschland, ab 2001); 
Wichtige historische Schlaglichter liefern außerdem themenbezogene Sammlungen wie das Projekt „Deutsche Geschichte in der Berliner Presse“ (1847-1990)51 oder das „Medienarchiv68“52 des Springer-Verlages.

- Was für viele Zeitungen gilt, gilt auch für die fotografische Überlieferung: zahlreiche visuelle Quellen sind zwar prinzipiell übers Internet verfügbar, aus kommerziellen und urheberrechtlichen Gründen aber allenfalls eingeschränkt recherchier- und nutzbar. Das gilt besonders für prominente private Bildagenturen.53 Zwar gewährend staatliche Archive und Bibliotheken per Internet Zugang zu umfassenden digitalen Bilddatenbanken wie etwa dem „Bildarchiv preußischer Kulturbesitz“, dem Bildarchiv des Bundesarchivs und des Deutschen Historischen Museums oder dem „Bildarchiv Austria“.54 Zudem existieren öffentliche Datenbanken zu Spezialthemen (Wiederaufbau, Erziehungs-, Kolonial-, Frauen-, Sozialgeschichte, linksalternative Bewegungen ${ }^{55}$ und digitale Bildarchive mit Schwerpunkt Bildende Kunst, Architektur- und Kulturgeschichte wie die „Deutsche Fotothek“ und der „Marburger Bildindex“; 56 auch englischsprachige Archive und

http://www.taz.de/digitaz/.archiv/suche.demo,1/suche?demo=1 (Die Tageszeitung, ab 1986); http://epaper.apps.welt.de/archiv/ (Die Welt, ab 2001).

${ }^{51}$ Vgl. http://www.zlb.de/projekte/millennium/.

52 Vgl. http://www.medienarchiv68.de/.

53 So auch für die für historische Themen eigentlich ertragreichen Agenturen dpa/Picture-Alliance (http://www.picture-alliance.com/), akg-images (http://www.akg-images.de/) oder http://www.frontalvision.com/historische_ddr_photos_g64.html.

54 Vgl. http://bpkgate.picturemaxx.com/webgate_cms/; http://www.bild.bundesarchiv.de/; http://www.dhm.de/sammlungen/bildarchiv/ bzw. http://www.dhm.de/datenbank/bildarchiv.html; http://www.bildarchivaustria.at/; http://ba.e-pics.ethz.ch. - Vgl. für die landesgeschichtliche Überlieferung z.B. http://www.datenmatrix.de/projekte/hdbg/bildarchiv/06_bildarchiv_uebersicht.php (Bildarchiv des Hauses der Bayerischen Geschichte); http://www.bsb-muenchen.de/Bilder.591.0.html (Bildarchiv der Bayerischen Staatsbibliothek); http://www.lwl.org/marsLWL/instance/ko.xhtml?oid=42611 (Bildarchiv des Landschaftsverbandes Westfalen-Lippe); http://www.lmz-bw.de/medien/bilddokumentation.html (Fotoarchiv des Landesmedienzentrums BadenWürttemberg). - Die Datenbanken sind z.T./perspektivisch auch über die Plattform Europeana erreichbar.

55 Vgl. http://www.datenmatrix.de/projekte/hdbg/bildarchiv/06_bildarchiv_allgemein.php (Hinweis auf Sammlung „Bayerisches Pressebild“ zum Wiederaufbau im Bildarchiv des Hauses der Bayerischen Geschichte); http://www.bbf.dipf.de/VirtuellesBildarchiv/ („Pictura Paedagogica Online“); http://www.ub.bildarchiv-dkg.uni-frankfurt.de/Bildprojekt/Bildsammlung/Bildsammlg.htm (Bildbestand der Frankfurter Kolonialgesellschaft); http://www.frauenmediaturm.de/recherche/bilddatenbank/ (im Aufbau); http://www.sozialarchiv.ch/archiv/recherche/datenbankbild-ton/ (Schweizerisches Sozialarchiv); http://umbruch-bildarchiv.de/bildarchiv/welcome.html.

56 Vgl. http://www.deutschefotothek.de/\#|home (auch erschlossen über die Europeana); http://www.bildindex.de/\#|home. - Auch Kunstmuseen unterhalten mitunter virtuelle Bildergalerien oder Ausstellungen; vgl. nur http://www.dresdengallery.com/; http://www.metmuseum.org/toah/; http://www.pinakothek.de/pinakothek-der-moderne/sammlungen/meisterwerke sowie Anm. 12. - Vereinzelt sind auch interessante kunsthistorische Ausstellungsprojekte wie „Die Farbe der Tränen. Der Erste Weltkrieg aus Sicht der Maler“ (http://www.memorial-caen.fr/10EVENT/EXPO1418/d/index2.html) im WWW dokumentiert. 
Geschichtsportale können wichtige Funde liefern. ${ }^{57}$ Eine uneingeschränkte Nutzung der Bildquellen ist jedoch auch in diesen Fällen meist nicht möglich. ${ }^{58}$

Freilich sind etliche, besonders die emblematischen Bilder deutscher Geschichte über Internetseiten von Museen, Bildungseinrichtungen oder Rundfunkanstalten oder „gemeinfreie“ Quellensammlungen wie bei Wikipedia und „zeno“ greifbar. Schwieriger ist die Suche nach digitalen oder digitalisierten Karten zur deutschen Geschichte, bei der man es mit verstreuten Angeboten von Landesarchiven, historischen Instituten oder landesgeschichtlichen Portalen zu tun hat. ${ }^{59}$

- Während Fotos noch relativ gut recherchierbar sind und „einschlägige“ historische Bilder an zahlreichen Stellen innerhalb „des Netzes“ reproduziert werden, sieht dies bei historischen Audio- und Videoquellen etwas anders aus. Nicht nur die Archive von Medienunternehmen und Sendeanstalten bleiben gegenüber der Internet-Öffentlichkeit abgeschirmt, auch zentrale medienhistorische Einrichtungen wie das Deutsche Rundfunkarchiv, das Deutsche Filmmuseum, das Filmarchiv im Bundesarchiv oder die Deutsche Kinemathek sind hinsichtlich audiovisueller Webpräsentationen aus urheberrechtlichen Gründen und wirtschaftlichen Motiven zurückhaltend. ${ }^{60}$ Wer beispielsweise im Netz nach

\footnotetext{
${ }^{77} \mathrm{Vgl}$. beispielsweise die Bildarchive des US Holocaust Memorial Museum (http://www.ushmm.org/research/collections/photo/) oder von Yad Vashem (http://collections.yadvashem.org/photosarchive/en-us/index-container.html) zum Thema Holocaust oder die Digitalisate der New York Public Library (http://digitalgallery.nypl.org/nypldigital/index.cfm).

58 Während private Bildarchive und -agenturen oft bereits den Zugang zu ihren Beständen beschränken, stellen viele staatliche Archive Voransichten der digitalisierten Bilder ins Netz, allerdings meist in beschränkter Auflösung oder mit Wasserzeichen versehen. Die Nutzung der hochauflösenden „Originale“ ist in der Regel erst auf Antrag und nach Erwerb von Nutzungsrechten möglich.

59 Vgl. den Überblick unter http://www.clio-online.de/site/lang_de/111/Default.aspx. Derzeit noch am brauchbarsten unter den reinen Kartenangeboten erscheinen: http://www.ieg-maps.unimainz.de/ (digitale Grundkarten); http://hgisg.geoinform.fh-mainz.de/mapbender22/i3mainz/sitemap/Uebersicht-thematischeKarten.php (dynamisch erzeugte thematische Karten, keine „Faksimiles"); http://www.davidrumsey.com/view/articles/view (mit Faksimiles historischer Deutschlandkarten); http://www.lib.utexas.edu/maps/germany.html und http://www.rootsweb.ancestry.com/ $\sim$ wggerman/map/index.htm (allerdings mit englischsprachiger Beschriftung).

60 Vgl. http://www.dra.de/; http://www.deutschesfilmmuseum.de; http://www.bundesarchiv.de/bundesarchive/organisation/abteilung_fa/index.html.de; http://www.deutsche-kinemathek.de/. Die ARD bietet unter http://www.swr.de/swr2/archivradio/ zwar wechselnde Streams aus dem Archiv der deutschen Rundfunkanstalten, aber keinen dauerhaften Fundus von Audioquellen. Der Sender Phoenix präsentiert Programmhöhepunkte der letzten zehn Jahre unter http://bibliothek.phoenix.de/; vom NDR-Politmagazin Panorama sind immerhin die Sendungen der letzten 50 Jahre zugänglich (http://daserste.ndr.de/panorama/archiv/index.html). - Ähnlich wie bei den bundesdeutschen Medien verhält es sich beim Filmarchiv Austria (http://filmarchiv.at/), dem österreichischen Rundfunk (http://orf.at/) oder dem Schweizer Filmarchiv (http://www.cinematheque.ch/d.html). Für die österreichische Geschichte liegen allerdings unter http://www.mediathek.at/ akustische Quellen sowie als „Akustische Chronik“eine multimediale Web-Ausstellung vor; vgl. außerdem http://www.austria-lexikon.at/af/Wissenssammlungen/AEIOU_Video_Album. Das Schweizer Fernsehen (http://www.sf.tv/archiv/) bietet einige kürzere Beiträge aus dem Fernsehen der 1950er bis 1990er Jahre an, allerdings i.d.R. in Schwyzerdütsch. Deutschsprachige Auszüge aus Schweizer Rundfunksendungen finden sich auch unter http://www.ideesuisse.ch/.
} 
NS-Propagandafilmen, den deutschen Wochenschauen ${ }^{61}$ oder filmhistorischen Klassikern der Weimarer Republik sucht, wird sie oft nur in geringer Auflösung, über Screenshots oder kurze Zitate greifen können - lässt man mal das bisweilen in einer rechtlichen Grauzone operierende „Sammelalbum“ YouTube (vgl. Erpel 2010: 158 bzw. Schultz 2008: 87) oder zweifelhafte, etwa von Rechtsextremen betriebene Seiten außen vor. Ausführlicher wird historisches Film- und Funkmaterial nur in wenigen Pilotprojekten zugänglich gemacht, etwa Politikerreden aus der Weimarer Republik oder Marshall-Plan-Filme der Alliierten durch das Deutsche Historische Museum ${ }^{62}$, Propagandasendungen des DDR-Rundfunks durch das Deutsche Rundfunkarchiv ${ }^{63}$ oder Radiomaterial zum Kalten Krieg. ${ }^{64}$

Wer historische Filmausschnitte oder Radiozitate für die landeskundliche oder geschichtsdidaktische Arbeit benötigt, wird somit derzeit vor allem in Internetausstellungen oder bei geschichtsbezogenen Websites von Bildungseinrichtungen und Rundfunkanstalten fündig. ${ }^{65}$ Dort trifft man allerdings häufig auf Kurzfilme oder Videoclips, die verschiedene Quellen (Fotos, Interviews, Filmaufnahmen)

61 Vgl. http://www.wochenschau-archiv.de/ (für die Zeit bis 1945; registrierte Nutzer können alle digital bereit gestellten Wochenschauen in reduzierter Auflösung kostenlos anschauen; Fassungen in höherer Auflösung erfordern Bezahlung) und http://www.deutsche-wochenschau.de/ (für die Zeit nach 1945; auf der Webseite können wenige ausgewählte Streams angesehen werden). - Viele der auf YouTube ursprünglich zu findenden Wochenschauen sind inzwischen gelöscht worden, nachdem das Bundesarchiv das Urheberrecht geltend gemacht hatte; ein Teil dieser Filme (etwa die der UFA) ist jedoch noch abzurufen. Auch im amerikanischen „Internet Archive“ http://www.archive.org/ kann man einige Wochenschauen ansehen, die dort als „Open Source“ klassifiziert sind. Die AustriaWochenschauen sind recherchierbar über http://www.europeanfilmgateway.eu/, wo man Links zu Streams erhält.

62 Vgl. http://www.dhm.de/sammlungen/zendok/weimar/ sowie http://www.dhm.de/filmarchiv/virtuelles-filmarchiv/die-filme/ (nur wenige in deutscher Sprache).

63 Vgl. http://1961.dra.de/ (Mauerbau aus Sicht von DDR-Hörfunk und -Fernsehen); http://1989.dra.de/ (Ausschnitte aus dem DDR-Fernsehen zur „Wende“ 1989/90). Vgl. auch die Projekte des Deutschen Rundfunkarchivs mit Filmen aus der Volkskammer (http://www.dra.de/dra/kooperationspartner/deutscher-bundestag.html) und zu den Sendungen des ,Schwarzen Kanals" (http:/ / sk.dra.de/, allerdings ohne Videos).

${ }^{64} \mathrm{Vgl}$. http://www.kalter-krieg-im-radio.de/. Vgl. auch die Filmclips in der Internetausstellung des Militärgeschichtlichen Forschungsamtes zur Geschichte der Bundeswehr unter http://50jahrebw.bundeswehr.de/mgfa/filme.htm oder einzelne Audio-/Rundfunkquellen unter http://www.mediaculture-online.de/Tonarchiv.804.0.html.

65 Vgl. die Hinweise in Anm. 30, 71 und Abschnitt 5. - Wer nicht einzelne Quellen, sondern allgemeines Informationsmaterial, Skripte oder Podcasts zu gelaufenen Sendungen sucht, wird u.a. fündig unter: http://www.ard.de/wissen/ (Tag „Geschichte“); http://zeitgeschichte.zdf.de; http://www.wissen.sf.tv/Dossiers/Historisch; $\quad$ http://www.spiegel.de/sptv/magazin/; http://www.planet-schule.de/sf/filme-online.php; http://www.br-online.de/bildung/databrd/index.htm bzw. http://www.br-online.de/br-alpha/schulfernsehen/index.xml; http://www.dradio.de/dlf/sendungen/reihen/ bzw. http://www.dradio.de/dkultur/sendungen/reihen/ (z.T. Audiopodcasts); http://www.kalenderblatt.de/ (Audiopodcasts, Deutsche Welle); http://www.hronline.de/website/specials/wissen/index.jsp bzw. http://www.hr-online.de/website/ radio/hr2/index.jsp?rubrik=28184 (Audiopodcasts); http://www.hr-online.de $/$ website $/ \mathrm{radio} / \mathrm{hr} 2 / \mathrm{in}$ dex.jsp?rubrik=2904 (Manuskripte); http://www.wdr.de/wissen/wdr_wissen/themen/geschichte/index.php5; http://www.br-online.de/bayern2/radiowissen/radiowissen-basics-unterricht-geschichte-ID1285936371339.xml; http://www.planet-wissen.de/politik_geschichte/index.jsp. 
kombinieren und erzählend kommentieren - was für den landeskundlichen Unterricht durchaus geeignet, in quellenkritischer Hinsicht aber nicht immer unproblematisch ist.

Damit sind auch medienspezifische Probleme der Quellenrecherche und Quellennutzung angesprochen. Die rasche technische Entwicklung und Dynamik des Netzes und die Tatsache, dass Internetangebote oft projektbezogen entwickelt und nicht kontinuierlich „gepflegt" werden, hat zur Folge, dass man bei der Suche oft noch auf ältere Angebote trifft, die die Potenziale des Netzes ungenutzt lassen, die lange Buchstabenkolonnen anstelle sinnvoll strukturierten Hypertextes bieten, Bilder nur als beiläufige Zutat liefern, audiovisuelle Quellen vernachlässigen, der Gestaltung nach anachronistisch wirken und die Erwartungen der Nutzer enttäuschen.

Darüber hinaus werden immer wieder interessante, in den einschlägigen Portalen verzeichnete Angebote aus dem Netz genommen oder so verschoben, dass sie unauffindbar werden, ${ }^{66}$ darunter aufschlussreiche virtuelle Ausstellungen zur Geschichte des Frauenstudiums in Deutschland, zu politisch Verfolgten während des Nationalsozialismus in Hamburg - oder das Projekt „Zeitenwende“ des Südwestdeutschen Rundfunks, das mit dem Anspruch eines „JahrhundertMemorials" historische Stimmen und Erinnerungen zum 20. Jahrhundert gesammelt hat, um dann nach zehn Jahren stillschweigend ,vom Netz genommen“ zu werden. ${ }^{67}$ Der kürzlich abgeschlossene neue Staatsvertrag für den öffentlichrechtlichen Rundfunk, der die Internetangebote der Sender streng limitiert, hat überdies zu zahlreichen Löschungen geführt. ${ }^{68}$

Bei der Interpretation der gefundenen Quellen ist nicht nur auf Transkriptionsfehler oder Bildbearbeitungen, sondern auf eine ausreichende Beschreibung zu achten. ${ }^{69}$ Während Archive, Museen, Bibliotheken die präsentierten Quellen meist

\footnotetext{
${ }^{66}$ Diese mangelnde „Langzeitstabilität“ des World Wide Web (s. Assmann 2004: 55f.) schränkt auch seine Leistungsfähigkeit als „kulturelles Gedächtnis“ der Gesellschaft (Pscheida 2010: 289) ein.

${ }^{67}$ Vgl. die entsprechenden Einträge und Verweise bei Clio online (etwa unter http://www.clio-online.de/site/lang_de/74/default.aspx), zu letztem Beispiel http://www.swr.de/swr2/zeitenwende/index_d.html.

68 Der seit Juni 2009 in Kraft befindliche 12. Rundfunkänderungsstaatsvertrag hat die Internetaktivitäten der öffentlich-rechtlichen Rundfunkanstalten zu Gunsten privater Anbieter stark eingeschränkt und verfügt, dass ein Großteil der Angebote nur noch begrenzte Zeit zur Verfügung stehen darf. Sendeinhalte und Begleitinformationen dürfen in der Regel nur sieben Tage im Web bereit gestellt werden bzw. müssen ein aufwändiges Prüfverfahren durchlaufen. Zwar können zeit- und kulturgeschichtliche Inhalte unbeschränkt und bildungsbezogene Inhalte für fünf Jahre im Netz bleiben, dennoch haben die Rundfunkanstalten im Anschluss an den Vertrag zahlreiche sendebegleitende Internetangebote gelöscht, darunter viele Seiten zu historischen Themen mit eingebundenen Quellen. Dieses flächendeckende „Depublizieren“ hat bei Historikern und Medienexperten heftige Kritik hervorgerufen (vgl. nur http://www.faz.net/artikel/C31013/depublizieren-die-leere-hinter-dem-link-30291159.html).

${ }^{69}$ Vgl. den Beitrag von Jürgen Nielsen-Sikora in diesem Band sowie Gruner 2009: 258; Näpel 2008: 94f.; zu Notwendigkeit und Ansatzpunkten einer speziellen „Quellenkritik“ für Internetseiten (als
} 
mit genauen Informationen zu Urheber, Datum und Entstehungskontext versehen, ist dies bei privat betriebenen Seiten mitunter nicht der Fall. So werden Bilder von militärischen Konflikten oder nationalsozialistischer Verfolgung bisweilen illustrativ oder zur Erzeugung von „Schauereffekten“ eingesetzt; und auch gut gemeinte Seiten thematisieren die Ausgrenzung der jüdischen Bevölkerung mit Fotos der Täterseite, ohne besonders darauf hinzuweisen, welche propagandistische Funktion diese hatten und wie weit sie den spezifischen Blick der Verfolger präsentieren.

Nicht unproblematisch sind Videoclips, die - nach den Gewohnheiten des im Fernsehen inzwischen üblichen „Histotainment ${ }^{\star 70}$ - Impressionen zu historischen Ereignissen oder Epochen in raschen Schnittfolgen aneinander reihen und mit suggestivem Sounddesign versehen, auf effektvolle Inszenierung, pathetische Überhöhung oder flotte Kommentierung setzen. Derartige Präsentationsformen ${ }^{71}$ können für einen thematischen Einstieg, für das historische Assoziieren und die Auseinandersetzung mit Geschichtsbildern und -darstellungen hilfreich sein, ${ }^{72}$ aber auch vorschnelle, vereinfachende Deutungen befördern und durch ihre Komposition den Weg zur einzelnen Quelle und deren spezifischer Aussage eher verstellen.

Auf der anderen Seite bedürfen nicht nur Bilder, sondern auch scheinbar evidente Texte wie Gesetze einer politik-, kultur- oder gesellschaftsgeschichtlichen Kontextualisierung, um sie adäquat zum Sprechen zu bringen. Gerade für die geschichts- und fremdsprachendidaktische Arbeit erscheint es deshalb ratsam, auf Angebote zurückzugreifen, die Quellen in historische Hintergrundinformationen einbetten und gegebenenfalls auf fachwissenschaftliche Deutungsangebote und Debatten verweisen. ${ }^{73}$ Solche Angebote haben überdies den Vorteil, die Masse der

Zusatz zur klassischen Quellenkritik) vgl. die Hinweise bei Eder; Fuchs 2005: 153ff.; Enderle 2002; Haber 2009: 194ff.; Marra 2005; Pfanzelter 2010.

70 Als Trendsetter in Deutschland wird hierfür meist die von Guido Knopp geleitete ZDFGeschichtsredaktion genannt, allerdings haben zu den neueren „Histotainment"-Formaten auch andere Sender und unterschiedliche Produktionsfirmen beigetragen (Meyer 2009a: 276). Zur Kritik der Fachwissenschaft an solchen Formen historischer Darstellung vgl. Bösch 2006; Fischer; Wirtz 2008; Handro 2009; Hein 2009a: 15f.; Kansteiner 2006: 109ff.; Keilbach 22010; Kolpatzik 2010; Meyer 2009c: 177f.; Näpel 2003; Paul 2010; Popp et al. 2010.

${ }^{71}$ Derartige historische Videoclips bieten meist die sendebegleitenden Websites der Rundfunkanstalten (vgl. Anm. 65). Angebote, die stark auf unterhaltende Aufbereitung, inszenatorische Elemente oder rasche Szenen- und Themenwechsel setzen, finden sich z.B. unter: http://diedeutschen.zdf.de/ZDFde/inhalt/12/0,1872,7272428,00.html?dr=1 (dazu auch Kolpatzik 2010); http://terra-x.zdf.de/ZDFde/inhalt/10/0,1872,8235178,00.html?dr=1; http://history.zdf.de/ZDFde/inhalt/26/0,1872,1020218,00.html?dr=1; http://www.dw-world.de/dw/0,12670,00.html; http://www.60xdeutschland.de/. Zurückhaltender in der Herangehensweise sind etwa die erläuternden Filme, die auf der Plattform http://www.deutschegeschichten.de/ abrufbar sind.

72 Vgl. auch die Anmerkungen im Beitrag von Uwe Koreik in diesem Band.

${ }^{73}$ Besonders zugespitzt beschreibt Gerhard Paul (2010: 24) die Gefahren einer Missachtung und Verfälschung historischer Zusammenhänge im Internet: „,Das Netz] offeriert [...] mit Wikipedia Informationsangebote, die dem aktuellen Forschungsstand meilenweit hinterherhinken oder ihn sogar konterkarieren oder wie bei Google und YouTube eine schier unübersehbare Anzahl von in der 
Überlieferung bereits auf aussagekräftige, didaktisch brauchbare Quellen reduziert zu haben. Diese Vorauswahl enthebt den Lehrenden jedoch nicht einer kritischen Reflexion. ${ }^{74}$ Obgleich viele der von Museen oder Bildungseinrichtungen gebotenen Präsentationen auf Neutralität, Sachlichkeit oder die geschichtsdidaktisch immer wieder geforderte Multiperspektivität achten, folgt die Darstellung doch nicht selten bestimmten historischen Perspektiven, Erzählrichtungen und Topoi. Landeskundliche Portale appellieren mitunter an ein modernisiertes Heimatgefühl, Museen und Medienangebote zur deutschen Geschichte folgen der „Meistererzählung" von der "geglückten Demokratie“ (vgl. Wolfrum 2006) oder „wiedergefundenen“ deutschen Nation (vgl. Kolpatzik 2010); Darstellungen, die den Ersten Weltkrieg als europäische „Urkatastrophe“ schildern, lassen die deutsche Kriegsschuld zurücktreten, und Websites, die jüdische Geschichte von der Shoah aus begreifen, erzählen mitunter eine nur aufs Katastrophische zugespitzte Verlustgeschichte. ${ }^{75}$

\section{Thematische Schwerpunkte und Defizite}

Inzwischen wird man zu vielen Themen einer historisch fundierten Landeskunde wie sie insbesondere von Uwe Koreik entworfen worden ist (Koreik 1995; Koreik 2010) - zumindest punktuell Quellen und Materialien im Internet finden. Gleichwohl lassen sich thematische und zeitliche Schwerpunkte und Lücken feststellen.

1. Wer nach epochenübergreifenden Angeboten zur deutschen Geschichte sucht, wird zunächst auf den Klassiker virtueller Ausstellungen verwiesen: das bereits Ende der 1990er Jahre eingerichtete „LeMO“ oder „Lebendige virtuelle Museum Online“, das 150 Jahre deutscher Geschichte abdeckt. Obgleich in der Aufmachung selbst schon historisch wirkend, mit einer etwas unübersichtlichen „Informationsarchitektur“, stark politikgeschichtlichen Ausrichtung und didaktischen Defiziten behaftet, ist es doch ein zentrales Arbeitsmittel für die Geschichtsvermittlung. Es bietet differenzierte zeitliche und thematische Zugriffe, zahlreiche interne Vernetzungen, ein ausführliches Glossar, Zeitzeugenerinnerungen, Bildleisten und wichtige Dokumente, Audio- wie Video-Quellen, von der Rede Wilhelms II. zum Kriegsbeginn 1914 bis zu Werbespots der 1950er

Regel entkontextualisierten, oftmals sogar nur in Ausschnitten wiedergegebenen Fotos und Filmen, die im wesentlichen nur die Schaulust der User, aber kein Aufklärungsbedürfnis bedienen und deren Herkunft, Kontext und Authentizität oft völlig unsicher sind." Vgl. auch Schmale 2010: 13f. sowie zu einer grundsätzlichen Kritik an Wikipedia und dessen unreflektierter Verwendung Cornelißen 2008; Grosch 2008: etwa 20ff.; Kümper 2010; Lorenz 2009a; Lorenz 2009 b.

74 ... im Sinne einer - bei Koreik (1995: 142ff.) oder Thimme (1996: 97ff.) beispielhaft vorgeführten kritischen Lehrmittelanalyse.

75 Vgl. hierzu auch Brautmeier et al. 2010; Jarausch; Sabrow 2002; Nonn 2007sowie die Befunde der Museumsforschung, auch wenn diese einen Trend zu vielschichtigen, reflexiven, erzählerisch und ästhetisch offeneren Ausstellungen erkennt (vgl. Beier-de Haan 2005; Hartung 2006; Pieper 2010; Thiemeyer 2010). 
Jahre. ${ }^{76}$ Zwei der Trägerorganisationen des LeMO, das Haus der Geschichte der Bundesrepublik Deutschland und das Deutsche Historische Museum, bieten darüber hinaus mit digitalen Ausstellungsarchiven Zugriff auf Darstellungen und Materialien zu Themen wie NS-Propaganda, Kriegsende, europäische Kulturbeziehungen, Kalter Krieg, deutsche Teilung oder DDR. ${ }^{77}$

Die bereits erwähnte Bundeszentrale für politische Bildung stellt den Nutzerinnen und Nutzern nicht nur etliche Themendossiers zur Verfügung, sondern ist auch an verschiedenen historischen Webportalen beteiligt, so an dem Projekt „Deutsche Geschichten“, das die Zeit zwischen 1890 und 2005 durch ausführliche Informationstexte sowie durch Audio- und Videodateien erschließt. ${ }^{78}$ $\mathrm{Zu}$ nennen sind darüber hinaus Angebote wie „100(0) Schlüsseldokumente zur deutschen Geschichte im 20. Jahrhundert“79, Portale wie die „Zentrale für Unterrichtsmedien" ${ }^{\text {"80 }}$ oder archivpädagogische Module - zumal diese mitunter frühere Phasen deutscher Geschichte (Spätmittelalter, Frühe Neuzeit, Absolutismus und Aufklärung) in den Blick nehmen und anhand ausgewählter Quellen thematische Längsschnitte ${ }^{81}$ zu setzen versuchen. Auch der Blick über die Grenzen der Bundesrepublik hinaus kann lohnen, einerseits wegen verschiedener Projekte von österreichischer Seite, ${ }^{82}$ zum anderen, weil auch angloamerikanische Websites bisweilen einen bündigen, seriösen Zugriff auf deutsche Quellen bieten. ${ }^{83}$

2. Die zentrale Rolle, welche die Auseinandersetzung mit dem Nationalsozialismus in der deutschen Erinnerungskultur seit zwei Jahrzehnten spielt, hat auch im Internet ein dichtes Geflecht von Informations- und Serviceangeboten entstehen lassen. Im Zentrum stehen die staats- und landeseigenen Gedenkstätten,

\footnotetext{
76 Vgl. http://www.dhm.de/lemo/home.html. Allerdings können einige ursprünglich präsentierte Filmquellen - vermutlich aus urheberrechtlichen Gründen - nicht mehr abgerufen werden. Zur Kritik an LeMO vgl. z.B. http://hsozkult.geschichte.hu-berlin.de/rezensionen/id=96\&type=rezausstellungen; Näpel 2008: 103; Pöppinghege 2010.

77 Vgl. http://www.hdg.de/bonn/ausstellungen/virtuell/; http://www.hdg.de/bonn/ausstellungen/wanderausstellungen/ (einzelne Pressematerialien); http://www.hdg.de/leipzig/ausstellungen/archiv/ (dto.); http://sint.hdg.de/sint/html/suche.html (Sammlungsdatenbank); http://www.dhm.de/ausstellungen/ausst.html (Ausstellungsarchiv mit kurzen Einführungen und z.T. Rundgängen).

${ }^{78}$ Vgl. http://www.deutschegeschichten.de/.

79 Vgl. http://mdzx.bib-bvb.de/cocoon/de1000dok/start.html (vornehmlich politikgeschichtlich, kaum audiovisuelle Quellen, mit ausführlicheren Erläuterungen) sowie Altrichter/Antipow 2009.

${ }^{80} \mathrm{Vgl}$. http://www.zum.de/psm/index1.php.

${ }^{81}$ Vgl. die Hinweise in Anm. 32. Thematische Längsschnitte betreffen beispielsweise die Geschichte der jüdischen Bevölkerung, der Jugend oder der Regime-/Systemwechsel in Deutschland.

$82 \mathrm{Vgl}$. die multimedialen Projekte unter Anm. 60; älter und landeskundlich weniger geeignet: http://www.uibk.ac.at/zeitgeschichte/zis/library/oesterreich-im-20.-jahrhundert/dokumente.

${ }^{83}$ Herauszuheben ist das differenzierte und nicht nur zeitlich breit angelegte, auch Quellen zur Kultur-, Sozial- oder Wirtschaftsgeschichte integrierende Angebot „German History Docs“ des Deutschen Historischen Instituts in Washington (http://germanhistorydocs.ghi-dc.org/Index.cfm?language $=$ german; wird kontinuierlich ausgebaut). Andere Sites wie das Internet Modern History Sourcebook (vgl. http://www.fordham.edu/halsall/mod/modsbook.html; ähnlich: http://www.csustan.edu/History/Faculty/Weikart/gerhist.htm) sind für den DaF-Unterricht schon deswegen nicht geeignet, weil sie die deutschen Quellen nur in englischer Übersetzung präsentieren.
} 
Museen und Dokumentationsstellen, die man über besondere Portale erreichen kann. ${ }^{84}$

Unter der Leitidee einer „Erziehung nach und über Auschwitz“ sind überdies Informationsplattformen und didaktisch orientierte Angebote wie „Shoa.de“, „Nationalsozialismus.de“, „Holocaust Referenz“, „Erinnern.at“ oder „Lernen aus der Geschichte entstanden“. 85 Hinzu kommen (teils verdiente, teils fragwürdige, auf den Schauder des Nazismus bauende) private Erinnerungsprojekte und Rechercheforen - und natürlich die einführenden Seiten der Rundfunkanstalten. Über diese Angebote lassen sich einzelne Quellen, Unterrichtsmaterialien oder weiterführende Internetpräsentationen zu Facetten der NS-Herrschaft aufspüren.

Neben den zentralen Dokumenten der NS-Herrschaft, Material zur NS-Propaganda $^{86}$ und den einschlägigen Bildern von Nationalsozialisten, Gleichschaltung und Terror finden sich im Netz auch verschiedene - geschichtsdidaktisch allerdings nur eingeschränkt brauchbare - Materialien zur Durchsetzung der NS-Herrschaft in einzelnen deutschen Gemeinden ${ }^{87}$, Milieus und Gruppen ${ }^{88}$, Biografien, Erinnerungsberichte und Darstellungen zu NS-Alltag, politischer Repression und Bombenkrieg 89 , lokal- und lebensgeschichtliche Quellen zu nationalsozialistischer Erziehung und Jugend im „Dritten Reich“,90 Zwangsarbeit und NS-Kranken-

\footnotetext{
${ }^{84}$ Vgl. die Hinweise in Anm. 28.

85 Vgl. http://www.shoa.de/; http://www.nationalsozialismus.de/; http://www.h-ref.de/ (allesamt private Initiativen/Angebote); http://lernen-aus-der-geschichte.de/ sowie http://www.fasena.de/ (Forschungs- und Arbeitsstelle „Erziehung nach/über Auschwitz").

86 Vgl. die Hinweise in Anm. 43 und 95.

87 Vgl. z.B. http://www.frankfurt1933-1945.de/; http://www.celle-im-nationalsozialismus.de/; http://www.lwg.uni-hannover.de/wiki/Hildesheim_im_Nationalsozialismus_-_Aspekte_der_Stadtgeschichte sowie http://www.memoryloops.net (München; virtuelles Denkmal, das auf Quellentexte zurückgreift).

${ }_{88}$ Vgl. http://www.kirche-christen-juden.org/ausstellung/ausstellung.html; http://www.zwangsarbeit-in-der-kirche.de/ns_kirche.php.

${ }^{99}$ Vgl. http://www.eg.nsdok.de/default.asp („Erlebte Geschichte“, Interviews mit Kölner Zeitzeuginnen und Zeitzeugen mit unterschiedlichsten Lebensgeschichten und einem Schwerpunkt auf Ausgrenzung und Verfolgung); http://www.lebensgeschichten.net/ (Biografiensammlung mit ergänzenden Dokumenten, Schwerpunkt Rheinland); http://www.zeitzeugengeschichte.de/ (Videound Audioclips zu Interviews mit Zeitzeugen der NS-Zeit, Schülerprojekt); http://www.resistancearchive.org/ („European Resistance Archive“ mit z.T. deutschsprachigen Videointerviews); http://www.dhm.de/lemo/forum/kollektives_gedaechtnis/index.html (Zeitzeugenberichte zur NS-Zeit); http://www.georg-elser.de/; http://www.gdw-berlin.de/themen/bereiche-d.php (Angebot der Gedenkstätte deutscher Widerstand); http://www.gegen-diktatur.de/ (Materialen zu Widerstand in Deutschland vor und nach 1945); http://www.doew.at/ausstellung/ (Internetpräsentation des Dokumentationsarchivs des österreichischen Widerstandes); http://www.widerstand.musin.de/ (zu Widerstand in München); http://www.ausdemleben.at/ (zur nationalsozialistischen Verfolgung der Homosexuellen in Wien); http://www.zeitzeugenforum.de/ (private Seite mit Erinnerungen an NSund „Kriegszeit").

${ }^{90}$ Vgl. z.B. http://www.museenkoeln.de/ausstellungen/nsd_0404_edelweiss/ (Internetausstellung zu unangepasstem Jugendverhalten in Köln); http://www.dhm.de/lemo/html/wk2/alltagsleben/klv/ (zur „,Kinderlandverschickung"); http://www.dhm.de/lemo/html/nazi/organisationen/jugend/; http://www.dhm.de/lemo/html/nazi/innenpolitik/bdm/; http://www.bdmhistory.com/ (jeweils einführende Materialien zu HJ/BDM); http://www.dhm.de/ausstellungen/bildberichterstatterin/
} 
mord, ${ }^{91}$ Judenverfolgung, Enteignung und Deportationen ${ }^{92}$ sowie Ausstellungen zur Geschichte der Konzentrationslager ${ }^{93}$.

Nachdem der Holocaust längst ein transnationales Thema, globales Symbol und universelle Metapher geworden ist (vgl. Assmann 2006: 255ff.; Eckel; Moisel 2008; Leggewie; Lang 2011; Levy; Sznaider 2001) und sich auch in Bezug auf den Zweiten Weltkrieg zunehmend europäische Perspektiven entwickeln (vgl. Arnold; Süß; Thießen 2009; Echternkamp; Martens 2007), lohnt auch der Blick auf die Träger der nicht-deutschsprachigen, speziell angloamerikanischen Erinnerungskultur. So hat Yad Vashem vor Kurzem sein Bildarchiv online gestellt; 94 und das US Holocaust Memorial Museum präsentiert eine Fotosammlung und mehrere Internetausstellungen, die unterschiedlichste Blickwinkel auf das NS-Regime eröffnen: von Aufzeichnungen Anne Franks über Schlaglichter der NSPropaganda bis zu einem Fotoalbum der SS in Auschwitz. ${ }^{95}$

(Fotos zur Erziehung von Frauen und Mädchen im NS-Regime); http://www.ub.uniheidelberg.de/helios/digi/nsfrauenwarte.html und http://www.calvin.edu/academic/cas/gpa/fw.htm (zur Zeitschrift der NS-Frauenschaft).

91 Vgl. www.zwangsarbeit.eu bzw. http://www.bundesarchiv.de/zwangsarbeit/ (Angebot des Bundesarchivs und der Stiftung „Erinnerung, Verantwortung und Zukunft“, mit Dokumenten, Fotografien, Lebenszeugnissen); http://www.ausstellung-zwangsarbeit.org/de/242/; http://www.Zwangsarbeit-archiv.de/ (Angebot der Stiftung „Erinnerung, Verantwortung und Zukunft“, der FU Berlin und des Deutschen Historischen Museums; mit Interviews in verschiedenen Sprachen; Registrierung erforderlich); http://www.kontakte-kontakty.de/deutsch/ns-opfer/freitagsbriefe/ (rückblickende Schilderungen früherer Zwangsarbeiter); http://www.historicum.net/themen/zwangsarbeit-rhein-erft-rur/ausstellung/; http://www.nrw-zwangsarbeit.de/; http://www.digada.de/zwangsarbeiter/uebersichtzwangsarbeiter.htm sowie http://www.denkzeichen.de/content/konzept/index.php?sid=c80d7b1823a809539e7e18419ac9b1f7\&flash=0 (zur NS-Tötungsanstalt Pirna-Sonnenstein); http://www.lpb-bw.de/publikationen/euthana/euthana.htm und http://www.landesarchiv-bw.de/stal/grafeneck/ (zur NS-Tötungsanstalt Grafeneck).

92 Vgl. http://www.blechner.com/german/index.html (zum Schicksal einer „Münchner Familie während des Holocaust“); http://www.chotzen.de/ („Lebenswege einer jüdischen Familie von 1914 bis heute“); http://www.uibk.ac.at/zeitgeschichte/zis/turteltaub/ („Die Geschichte einer jüdischen österreichischen Familie"); http://www.forschungsgesellschaft.at/emigration/index_d.htm („Vertrieben. Erinnerungen burgenländischer Juden“); http://www.ghwk.de/deut/ausstellung2006.htm (Angebot der Gedenkstätte Haus der Wannsee-Konferenz); http://www.dhm.de/ausstellungen/holocaust/ausstellung.htm; http://hannoverscher-bahnhof.hamburg.de/; http://www.archiv.sachsen.de/6078.htm („Die Verfolgung Leipziger Juden 1938/39“); vgl. auch http://www.iff.ac.at/inventarisiert/inventar_de.html.

${ }^{93}$ Initiative in dieser Hinsicht entwickelt derzeit vor allem die Gedenkstätte Buchenwald; vgl. http://www.buchenwald.de/fotoarchive/buchenwald/; http://www.buchenwald.de/fotoausstellung/; http://www.topfundsoehne.de. Für die Gedenkstätte Sachsenhausen vgl. http://www.stiftung-bg.de/gums/de/index.htm („Die Fälscherwerkstatt im KZ Sachsenhausen“); http://www.stiftung-bg.de/kz-oranienburg/ („Die politischen Häftlinge des KZ Oranienburg"). Vgl. auch http://videoarchiv-ravensbrueck.de/ und weitere Berichte von Überlebenden des Frauenkonzentrationslagers unter http://www.bpb.de.

${ }_{94}$ Vgl. http:// collections.yadvashem.org/photosarchive/en-us/photos.html.

95 Vgl. http://www.ushmm.org/museum/exhibit/online/af/htmlsite/index.html; http://www.ushmm.org/propaganda/exhibit.html\#/gallery/; http://www.ushmm.org/museum/exhibit/online/ssalbum/; Weiteres unter http://www.ushmm.org/museum/exhibit/online/. Zu den von Yad Vashem entwickelten Onlineausstellungen vgl. http://www1.yadvashem.org/yv/en/exhibitions/index.asp. Vgl. auch den Onlineauftritt des Anne Frank Hauses (unter http://www.annefrank.org- 
Mit der Historisierung der NS-Zeit und ihrer Einbettung in die längeren Linien deutscher Geschichte ist zuletzt auch die Nachgeschichte der NS-Herrschaft, die gescheiterte Entnazifizierung, vielfach blockierte oder selektive „Vergangenheitsbewältigung“ sowie die verstärkte Aufarbeitung seit den 1960er Jahren zum Thema gemacht worden (einführend Fischer; Lorenz 2007; Reichel 22007; Reichel; Schmid; Steinbach 2009). Die NS-Geschichte wird demnach auch im Internet mit Quellen zu den Nürnberger Prozessen ${ }^{96}$ oder der berühmten Weizsäcker-Rede von $1985^{97}$ - zur bundesdeutschen und internationalen Geschichte hin geöffnet. Eine perspektivische Erweiterung erlauben zudem Materialen zur jüdischen Geschichte. ${ }^{98}$ Sie ermöglichen einmal, die nationalsozialistische Judenverfolgung in die lange Geschichte des deutschen - und abendländischen - Antisemitismus

/de) sowie http://germanhistorydocs.ghi-dc.org/section.cfm?section_id=13. - Die zahlreichen amerikanischen Seiten zum Thema präsentieren oft Quellen, Texte jedoch meist nur in englischer Übersetzung; umfangreichere Bildsammlungen finden sich etwa unter http://fcit.coedu.usf.edu/holocaust/resource/gallery/gallery.htm\#1 („A Teacher's Guide to the Holocaust").

96 Vgl. http://www.zeno.org/Geschichte/M/Der+N\%C3\%BCrnberger+Proze $\%$ C3\%9F (deutschsprachige Fassung); http://nuremberg.law.harvard.edu/php/docs_swi.php?DI=1\&text=overview (englischsprachige Fassung mit Bildmaterial); http://www.ushmm.org/museum/exhibit/focus/warcrimetrials/ (Bildmaterial); http://www.nuernberg.de/internet/museen/bildarchiv/prozesse.html (dto.) bzw. http://www.memorium-nuernberg.de/. Die meisten Internetmaterialien zu NS-Prozessen liegen derzeit in englischer Sprache vor (Gruner 2009); zu deutschen Prozessen einige Quellen unter http://www1.jur.uva.nl/junsv/Lesen.htm (Auszüge aus Urteilen); http://einestages.spiegel.de/static/topicalbumbackground/1853/als_westdeutschland_aufwachte.html (Fotos); http://www.landesarchiv-bw.de/web/43377 (Tonbandaufnahme einer Urteilsverkündigung). Eine multimediale Präsentation des Hessischen Rundfunks zum Frankfurter Auschwitz-Prozess ist unter http://www.hr-online.de/website/static/spezial/auschwitzprozess/index.html zu finden. - Einige Quellen zum Thema „Entnazifizierung“ bietet http://germanhistorydocs.ghi-dc.org/sub_docs.cfm?section_id=14, einige Zeitzeugenberichte zur Nachkriegszeit http://www.dhm.de/lemo/forum/kollektives_gedaechtnis/index.html.

97 Vgl. http://www.bundespraesident.de/SharedDocs/Reden/DE/Richard-von-Weizsaecker/Reden/1985/05/19850508_Rede.html und http://www.mediaculture-online.de/fileadmin/mp3s/weizsaecker_geschichte.mp3. Vgl. auch http://www.20-juli-44.de/reden.php. - Zur Erinnerung an den Nationalsozialismus vgl. auch http://www.bpb.de/themen/DU8MZJ,0,0,Geschichte_und_Erinnerung.html und http://www.bpb.de/themen/S82KDR,0,0,Bildergalerie.html.

98 Vgl. die Hinweise in Anm. 92 sowie die Präsentationen unter http://www.jmberlin.de/ (Jüdisches Museum Berlin); http://www.juedischesmuseum.de/ (Frankfurt/Main) und die archivpädagogischen Angebote unter http://www.digam.net/?str=247 (Deutsch-jüdische Geschichte vom Mittelalter bis zur Gegenwart); http://www.digada.de/juden/uebersichtjuden.htm (Jüdisches Leben in Südhessen). Zur jüdischen Publizistik http://www.literatur-des-judentums.de (digitalisierte jiddische Drucke); http://www.compactmemory.de/ (Jüdische Periodika des 19. und frühen 20. Jahrhunderts); http://sammlungen.ub.uni-frankfurt.de/freimann/ (Judaica-Sammlung); http://deposit.d-nb.de/online/jued/jued.htm (Jüdische Zeitungen aus NS-Deutschland und davor). Vgl. auch http://www.synagogen.info/ (Synagogen Internetarchiv mit virtuellen Darstellungen zerstörter Synagogen; dazu Hein 2009a: 155ff.); http://spurensuche.steinheim-institut.org/ (Informationsangebot zu jüdischen Friedhöfen); http://www.vor-dem-holocaust.de/ (Jüdisches Alltagsleben in Hessen in Fotografien); http://www.ifs.tu-darmstadt.de/fileadmin/ueberlebende-zeilsheim/index.html (Jüdische Überlebende in Frankfurt-Zeilsheim); http://www.juedisches-leben.net (Internetportal ",Jüdisches Leben in Europa jenseits der Metropolen“); http://www.deutschefotothek.de/ (mit Themenfilter ,Judentum“/,jüdisch"); zur epochenübergreifenden Geschichte des Antisemitismus auch http://www.antisemitismus-anhalt.de/zeitreisen.html (Spurensuche zum Antisemitismus in Anhalt); http://www.buehler-hd.de/gnet/neuzeit/antisem/hd/doku.htm (,Abgestempelt. Judenfeindliche Postkarten“). 
einzuordnen; andererseits helfen sie, jüdische Geschichte nicht auf eine Opfergeschichte zu verengen und jüdische Kultur als einen Teil deutscher Geschichte zu verstehen.

3. Wenn auch der Nationalsozialismus eine prägende Rolle in der Erinnerungskultur und im „virtuellen historischen Raum“ spielt, hat sich in den letzten Jahren doch ein starkes Gegengewicht gebildet mit der Geschichte der DDR, des deutschdeutschen Systemkonflikts und der Wiedervereinigung (vgl. Behrens; Ciupke; Reichling 2009; Sabrow 2009; Sabrow 2010). Seit Mitte der 1990er Jahre haben politische Stiftungen, Archive und Sendeanstalten hierzu empfehlenswerte Internetplattformen geschaffen, so zum 17. Juni 1953, zum Mauerbau 1961, zur „Chronik der Mauer“ oder „Chronik der Wende“.99 Sie verknüpfen einführende Texte zu Diktatur, Opposition und Systemwandel mit Fotos, Dokumenten, Umfragen, Erinnerungsberichten, O-Tönen, Rundfunk- und Fernsehclips und bieten so zahlreiche Ansatzpunkte für die historisch-politische Landeskunde. Ergänzt werden solche vielschichtigen Angebote durch kleinere oder thematisch enger abgesteckte Projekte, die alternative Bilder und Deutungen zu den zentralen Ereignissen, Figuren und Szenen der DDR-Geschichte liefern, etwa zu Erziehung und Jugendopposition in der DDR, ${ }^{100}$ zur Grenz- und Fluchtgeschichte oder zu Fotografien aus dem Alltag der Diktatur. ${ }^{101}$

\footnotetext{
${ }^{99}$ Vgl. http://www.17juni53.de/home/index.html; http://1961.dra.de/ (,Eine Woche im August... Der Mauerbau 1961 im Hörfunk und Fernsehen der DDR"); http://www.chronik-der-mauer.de/; http://1989.dra.de/ („Wende-Zeiten. Bilder, Töne, Kommentare aus dem DDR-Fernsehen“); http://www.chronikderwende.de/ sowie dazu Alavi 2008, die den Materialreichtum der Seite lobt, allerdings auch auf kritische Punkte hinweist (eingeschränkte Übersichtlichkeit des Angebots, analytische Herangehensweise, wissenschaftliche Sprache, notwendiges Vorwissen) und daraus die Notwendigkeit einer weiteren Didaktisierung ableitet. Umfassende, vor allem auf Audio- und Videodateien setzende Angebote finden sich auch unter http://www.mdr.de/damals/index.html („Damals im Osten. Mitteldeutschland - 1945 bis heute“) und http://www.mdr.de/www3/euregeschichte/ (Schulprojekt zur DDR-Geschichte); weitere Nachrichten- und Zeitzeugenclips unter http://unsere-geschichte.zdf.de/ZDFde/inhalt/19/0,1872,7112211,00.html (zur „Wiedervereinigung“ 1989/90); Informationsangebote mit ergänzenden Quellen und Zeitzeugenberichten unter http://www.stiftung-aufarbeitung.de/50-jahrestag-der-berliner-mauer-1651.html; $\quad$ http://revolution89.de/ und http://www.deinegeschichte.de/; weitere Erinnerungsberichte unter http://www.mein-herbst-89.de/; http://www.dhm.de/lemo/forum/kollektives_gedaechtnis/index.html.

100 Vgl. die umfassende, zahlreiche Zeitzeugeninterviews präsentierende Website http://www.jugendopposition.de/index.php?id=1. - Die unter http://www.fachportal-paedagogik.de/filme/ vorgestellte Sammlung von Schulfilmen aus der DDR ist leider nur für registrierte Nutzer mit wissenschaftlichem Interesse zugänglich.

101 Vgl. http://www.berliner-mauer-gedenkstaette.de; http://www.grenzerinnerungen.de/ (persönliche Berichte und Bilder); http://www.mauerfotos.de (privates Internet-Fotoarchiv); http://www.berliner-mauer.de/. Weitere Zeitzeugen-/Erinnerungsberichte und begleitende Fotos in dem umfangreichen, von der Bundeszentrale für politische Bildung unterstützten Portal http://www.wirwaren-so-frei.de/index.php sowie auf der privaten Website http://www.ddr-zeitzeugen.de/index.html und unter http://www.zeitzeugenforum.de/. Zur Bildgeschichte der DDR vgl. auch http://www.ddr-bilder.de/; http://www.ddr-fotografie-riemann.de/html/ddr-galerie.html; http://www.dhm.de/ausstellungen/pdr/homep.htm (Bilder aus dem „Palast der Republik“). Links zu den DDRMuseen und -Gedenkstätten unter http://www.stiftung-aufarbeitung.de/erinnerungsorte-1176.html.
} 
4. Wer landeskundlich eine Fixierung auf Deutschland als „Land der Diktaturen“"vermeiden will, muss derzeit bei der Quellenrecherche noch mit mehr Aufwand rechnen. Im Zuge der Selbsthistorisierung der Bundesrepublik und zeithistorischer Debatten ${ }^{102}$ sind in den letzten Jahren aber auch umfangreichere multimediale Präsentationen zur westdeutschen Geschichte seit $1945^{103}$ entstanden. Hinzu kommen Internetdossiers, die interessantes Material zu Einzelaspekten bieten: zu Parteien und Politikfeldern wie der Außen- und „Neuen Ostpolitk“104, zu Themen wie Nachkriegszeit, Wiederaufbau und Wirtschaftswunder ${ }^{105}$, „Kaltem Krieg“ und Deutscher Teilung ${ }^{106}$, zu Terrorismus ${ }^{107}$ oder „'68“, APO und den Neuen Sozialen Bewegungen ${ }^{108}$.

\begin{abstract}
102 Vgl. die Hinweise in Anm. 6 sowie Görtemaker 2004; Schildt; Siegfried 2009; Wolfrum 2006.
103 Vgl. nur http://www.60xdeutschland.de/ (umfassendes Angebot der ARD und der Bundeszentrale für politische Bildung mit Videoclips und Hörfunkbeiträgen; dazu auch Handro 2010: 208ff.); http://www.dw-world.de/dw/0,12670,00.html (ähnliches Angebot der Deutschen Welle zu „60 Jahre Bundesrepublik“"); http://www.dpa.de/60-deutsche-Jahre.506.0.html (Artikel verschiedener Zeitungen).
\end{abstract}

104 Vgl. http://www.kas.de/wf/de/71.9060/ (Geschichte der CDU auf den Seiten der Konrad-Adenauer-Stiftung); http://www.fes.de/archiv/adsd_neu/inhalt/downloads/geschichte_spd.htm; http://www.fes.de/hfz/arbeiterbewegung/epochen/bundesrepublik-deutschland-1949-1989/kollektion (mit Links auf die Pressemitteilungen, Parteitagsprotokolle und den Pressedienst der SPD); zur „Neuen Ostpolitik“ beispielsweise http://library.fes.de/library/netzquelle/ostpolitik/index.html (Materialien der Friedrich-Ebert-Stiftung); http://germanhistorydocs.ghi-dc.org; http://www.auswaertiges-amt.de/sid_168AE7F8700E71F717C1C6573326CBF4/DE/AAmt/PolitischesArchiv-

/HistorischeDokumente/Ostpolitik_node.html.

105 Vgl. http://www.hdg.de/lemo/html/Nachkriegsjahre/index.html; http://germanhistorydocs.ghidc.org/sub_docs.cfm?section_id=14\&language=german; http://www.dhm.de/filmarchiv/virtuelles-filmarchiv/die-filme/ (Marshall-Plan-Filme); http://www.parlamentarischerrat.de/; http://www.digada.de/nachkriegszeit/uebersichtnachkriegszeit.htm (Nachkriegszeit in Hessen); http://www.lwl.org/LWL/Kultur/Aufbau_West/home/ (,Neubeginn zwischen Vertreibung und Wirtschaftswunder" in Westfalen); http://www.hdbg.de/wanderausstellung-wiederaufbau/wiederaufbau_themen.php (Bayern).

106 Vgl. nur http://www.bpb.de/themen/KGBNU7,0,0,Deutsche_Teilung_Deutsche_Einheit.html (Überblick der Bundeszentrale für politische Bildung mit Materialien und weiteren Nachweisen) und http://www.kalter-krieg-im-radio.de/ sowie die Hinweise in Anm. 100.

107 Vgl. http://www.bpb.de/themen/TSS56U,0,0,Die_Geschichte_der_RAF.html (Überblick der Bundeszentrale für politische Bildung); http://www.wdr.de/themen/politik/deutschland/deutscher_herbst/uebersicht.jhtml?rubrikenstyle=kultur (als Beispiel für die zahlreichen Themenseiten der Rundfunkanstalten); http://www.hdg.de/lemo/html/DasGeteilteDeutschland/NeueHerausforderungen/Linksterrorismus/; http://labourhistory.net/raf/index-de.php (Dokumentensammlung); http://www.swr.de/swr2/wissen/specials/-/id=661214/nid=661214/ did=2414272/2q12qs/index.html (,Tondokumente von den RAF-Prozessen“ in Stammheim). Überblick über die verschiedenen - meist noch aktualisierten - Dossiers von Rundfunksendern und Tageszeitungen unter http://www.zeitgeschichte-online.de/site/40208316/default.aspx.

108 Vgl. z.B. http://www.bpb.de/themen/UEZYL5,0,0,Die_68erBewegung.html (Überblick der Bundeszentrale für politische Bildung zur „68er-Bewegung“" mit Materialien und weiterführenden Hinweisen); http://www.medienarchiv68.de/; http://www.glasnost.de/hist/apo/ (Quellen und Texte zur Geschichte der Außerparlamentarischen Opposition); http://www.fr-online.de/politik/spezials/zeitgeschichte/1968/-/1477444/1477444/-/index.html (Dossier mit zahlreichen Fotos); http://service.tagesschau.de/multimedia-box/index.php?id=1968 (Fernsehausschnitte); http://germanhistorydocs.ghi-dc.org/sub_doclist.cfm?sub_id=34\&section_id=15\&language=german; http://www.hr-online.de/website/specials/68er/index.jsp (,Frankfurt 1968 - Ein Stadtrundgang 
Ein für die interkulturelle Bildungsarbeit wichtiger Aspekt ist darüber hinaus die Migrationsgeschichte, die seit einigen Jahren nicht nur wissenschaftlich starke Beachtung findet, sondern integrales Thema historischer Bildungsarbeit geworden ist (vgl. Lange 2008; Leggewie; Lang 2011: 162ff.; Motte; Ohliger 2004). Das betrifft das immer noch geschichtspolitisch umkämpfte Thema der Vertreibungen, ${ }^{109}$ vor allem aber die Geschichte der BRD als „Gastarbeiter-“ und „Einwanderungsland“. Sie hat in den letzten Jahren erste Würdigung in Websites gefunden, die Ankunft, Ausgrenzung und Integration vor allem alltagsnah und lebensgeschichtlich nacherzählen. ${ }^{110}$ Die verstärkte Aufmerksamkeit für die Migrationsgeschichte hat überdies die deutsche Ein- und Auswanderung des 19. und frühen 20. Jahrhunderts in den Blick rücken lassen. ${ }^{111}$

nach 40 Jahren“). - Zur ,grünen Bewegung“ vgl. http://www.boell.de/stiftung/archiv/archiv4285.html (Materialsammlung zur Partei von Seiten der Heinrich-Böll-Stiftung). Zur ,neuen Frauenbewegung“ vgl. http://www.bpb.de/themen/KYOE75,0,0,Frauenbewegung.html (Überblick der Bundeszentrale für politische Bildung); http://www.hdg.de/lemo/html/DasGeteilteDeutschland/KontinuitaetUndWandel/UnruhigeJahre/neueFrauenbewegung.html; das Angebot auf germanhistorydocs.ghi-dc.org sowie http://www.frauenmediaturm.de/recherche/ („EMMA“-Archiv und Bilddatenbank, allerdings erst im Aufbau). Auch zur „ersten Frauenbewegung“ des späten 19./ frühen 20. Jahrhunderts gibt es dort punktuell Internetmaterialien.

109 Vgl. zur jüngeren Debatte http://www.zeitgeschichte-online.de/site/40208192/default.aspx. Trotz der Intensität der Auseinandersetzung stellt das Internet bisher nur relativ wenig historische Materialien und Quellen zu den Vertreibungen nach 1945 zur Verfügung. Neben den wegen ihrer geschichtspolitischen Ausrichtung nicht unproblematischen Seiten der Vertriebenenverbände finden sich überschaubare Quellenhinweise etwa unter http://library.fes.de/library/netzquelle/zwangsmigration/index.html; deutschland/migrationen/rooms/0504.htm; http://www.dhm.de/ausstellungen/zuwanderungslandjahre/DasEndeAlsAnfang/fluchtUndVertreibung.html; http://www.hdg.de/lemo/html/Nachkriegs(Flüchtlinge und Vertriebene in Hessen); http://www.lwl.org/LWL/Kultur/Aufbau_West/flucht/; http://www.hdbg.de/integration/de/index.htm. Knapper Einblick in die Ausstellung des „Bundes der Vertriebenen“ zu Zwangsmigrationen im 20. Jahrhundert unter http:/ / erzwungenewege.z-g-v.de; Zeitzeugeninterviews zum Thema „Flucht und Vertreibung“ aus verschiedenen europäischen Ländern bietet http://www.the-unwanted.com/.

110 Wesentlicher Impulsgeber war hierbei das seit 1990 aktive (zunächst auf die Immigration aus der Türkei fokussierte) „Dokumentationszentrum und Museum über die Migration in Deutschland“ (vgl. http://www.domid.org/index.html), dessen Ausstellungen zum Thema jedoch nur teilweise im Internet verfügbar sind. - Wichtige migrationsgeschichtliche Präsentationen finden sich jedoch unter http://www.angekommen.com/iberer/index.html (Sammlung von Biografien mit Interviewausschnitten); http://www.angekommen.com/italiener/index.html (dto.); http://www.migrationaudio-archiv.de/ (Sammlung mit längeren lebensgeschichtlichen Interviews); http://www.einfamilienalbum.de/ (Familiengeschichte von Deutschen und Einwanderern von 1888 bis 1999); http://lebenswege.rlp.de/lebenswege/ (Biografien, z.T. mit Audiodateien). Neuere Materialien und Quellen bieten des Weiteren http://lebenswege.rlp.de/sonderausstellungen/50-jahre-anwerbeabkommen-deutschland-griechenland/; http://lebenswege.rlp.de/sonderausstellungen/50-jahre-anwerbeabkommen-deutschland-spanien/ (zur Zuwanderung nach Rheinland-Pfalz); http://www.muelheim-ruhr.de/cms/migration-geschichte.html (lokalgeschichtliches Angebot); http://www.museum-neukoelln.de/ausstellungen-99-neukoelln.php (Präsentation migrationsgeschichtlich aufschlussreicher Objekte). $\mathrm{Zu}$ migrationsgeschichtlichen Ausstellungen vgl. allg. http://www.lwl.org/LWL/Kultur/wim/portal/S/hannover/ort/migration/exponat/.

111 Vgl. neben http://www.angekommen.com/italiener/index.html http://www.migrationsroute. nrw.de/ (zu Erinnerungsorten der Migrationsgeschichte in NRW); http://www.dhm.de/ausstellungen/zuwanderungsland-deutschland/migrationen/index.html; http://www.hdbg.de/auswande- 
5. Migrationsgeschichtliche Quellen bieten wichtige Ansatzpunkte, um Identitäten, Identitätspolitiken, historische Selbst- und Fremdzuschreibungen zu thematisieren, wären indes nicht die einzigen Materialien, die hierfür in Frage kommen. Andere Ressourcen - etwa zur Außen- und Kulturpolitik, zu den „Bildern des Anderen" in Schulbüchern, zu Tourismus und Reiseliteratur - sind allerdings bisher nur vereinzelt im Internet auszumachen. Dieser Befund verwundert etwas angesichts der teilweise recht intensiven kulturdiplomatischen Bemühungen und Versöhnungsinitiativen nach dem Zweiten Weltkrieg und des in den letzten Jahren wiederbelebten Diskurses über die koloniale Vergangenheit Deutschlands (vgl. Kundrus 2003; Laak 2005). Die in den letzten Jahren intensivierten Debatten um eine europäische Erinnerungskultur (vgl. Assmann 2006: 250ff.; Kühberger; Sedmak 2009; Leggewie; Lang 2011) könnten jedoch dazu beitragen, dass die Beziehungsgeschichte der Nationen, Länder und Kulturen im Internet bald stärkere Beachtung findet.

Immerhin liegen bereits wenige Angebote zur deutschen Kolonialgeschichte vor $^{112}$ - sowie zur Beziehungsgeschichte von Deutschland und seinen europäischen Nachbarn, vor allem zu Polen. ${ }^{113}$ Auch ein Projekt wie das „Virtuelle Museum", das die deutsch-dänische Grenzregion in den Blick nimmt, zeigt, dass es auf diesem Gebiet Entwicklungspotenziale gibt. ${ }^{114}$ Das gilt auch für die deutschfranzösischen Beziehungen, die bereits seit Jahrzehnten bildungspolitisch, kulturhistorisch und geschichtsdidaktisch reflektiert und ,gepflegt“" werden (vgl. Dalmas 2010). Hierzu lassen sich im Internet zwar regierungsamtliche, wissenschaftliche

rung/deutsch/index2.htm (Auswanderung aus Bayern nach Amerika seit 1683); http://www.hamburg.de/bilder/hamburg-historisch (Bilder und Texte zum Auswandererhafen Hamburg).

112 Vgl. http://www.bpb.de/themen/KB4UAN,0,0,Ein_Platz_an_der_afrikanischen_Sonne.html (Überblick der Bundeszentrale für politische Bildung); http://www.dhm.de/lemo/html/kaiserreich/aussenpolitik/kolonien/index.html (Einstieg zur Kolonialpolitik des deutschen Kaiserreiches); http://www.bundesarchiv.de/oeffentlichkeitsarbeit/bilder_dokumente/index.html.de (unter Kategorie „Kaiserreich“ Quellendossiers zum Krieg gegen die Herero 1904, zu Kolonialkarten, zu den „Musterkolonien“ Kiautschou und Samoa, zum „Boxeraufstand“); http://www.zum.de/psm/imperialismus/primaer.php (Quellen/Transkripte zur deutschen Kolonialpolitik im 19./frühen 20. Jahrhundert); http://www.ub.bildarchiv-dkg.uni-frankfurt.de/Bildprojekt/Bildsammlung/Bildsammlg.htm (Bildbestand der Deutschen Kolonialgesellschaft); http://www.ub.bildarchiv-dkg.uni-frankfurt.de/Bildprojekt/Lexikon/lexikon.htm (Transkript des Deutschen Koloniallexikons von 1920); http://www.dhm.de/ausstellungen/namibia/rundgang.htm. Zu den gegenwärtig noch sichtbaren Spuren des Kolonialismus vgl. http://www.koloniale-spuren.de/ (zu Hannover); http://www.afrika-hamburg.de; http://www.freiburg-postkolonial.de; http://www.kopfwelten.org/kp/ („,Köln postkolonial“).

113 Vgl. http://www.bpb.de/themen/7L1YH7,0,0,Deutschpolnische_Beziehungen.html (Überblick); http://www.deutsche-und-polen.de/ (historischer Überblick mit Bild- und Videomaterial); http://library.fes.de/library/netzquelle/deutsch-polnisch/index.html (Materialien der FriedrichEbert-Stiftung); http://www.deutschland-polen.diplo.de/Vertretung/deutschland-polen/de/Startseite.html (Präsentation des Auswärtigen Amtes, weitgehend gegenwartsorientiert). - Zu den deutschrussischen Beziehungen vgl. http://www.unsererussen.de/ausstellung.html; http://www.hdg.de/bonn/ausstellungen/archiv/2003/spuren-sledy-deutsche-und-russen-in-der-geschichte/; zu den deutsch-skandinavischen Beziehungen im 19. Jahrhundert http://www.dhm.de/ausstellungen/wahlverwandtschaft/.

${ }_{114}$ Vgl. http://www.vimu.info/ sowie Danker; Schwabe 2008b und Nissen 2008. 
und didaktische Materialen finden. ${ }^{115}$ Wichtige Aspekte sind aber oft nur über thematisch anders gelagerte Seiten zu erfassen. So kann man z.B. die Wahrnehmungen der Kriegsgegner im Ersten Weltkrieg über bild- und propagandageschichtliche Sammlungen, ${ }^{116}$ die deutsche Kampagne gegen die französischen Kolonialsoldaten über regional- oder lokalhistorische Seiten ${ }^{117}$ oder die Verwandlung der „Erbfeinde“ zu Partnern im Rahmen europäischer Sicherheitspolitik über eine Präsentation zur deutschen Militärgeschichte thematisieren. ${ }^{118}$

6. Material zu den „Aushängeschildern“ deutscher Kultur ist aufgrund intensiver Forschung und Bildungsarbeit, von Traditionspflege und Standortmarketing meist problemlos zu finden - ob zu den ,großen Männern“ Adenauer, ${ }^{119}$ Bismarck ${ }^{120}$, Ebert oder Brandt, ${ }^{121}$ Luther, Goethe, Bach oder Beethoven, ${ }^{122}$ ob zum Kölner Dom, der Paulskirche oder Neuschwanstein. ${ }^{123}$ Weitaus schwieriger ist es hingegen, wenn es um die historische Entstehung und Ausprägung von

115 Vgl. http://www.deuframat.de/ („,Deutsch-französische Materialien für den Geschichts- und Geographieunterricht“, Gemeinschaftsprojekt des Bundeskanzleramtes und des Georg-Eckert-Instituts für internationale Schulbuchforschung); http://www.fplusd.org/kultur-und-alltagsleben/politikund-geschichte/ (Sprachenportal der Außenministerien); http://www.france-allemagne.fr/Geschichte-der-deutsch,1501.html (Portal der Außenministerien); http://www.leforum.de/de/de-traites.htm; http://www.hdg.de/lemo/html/DasGeteilteDeutschland/DieZuspitzungDesKaltenKrieges/Tei-

lungDeutschlands/deutschFranzoesischeBeziehungen.html. Die Website zur Ausstellung „Fremde? Bilder von den ,Anderen in Deutschland und Frankreich seit 1871“ (http://www.dhm.de/ausstellungen/fremde/ausstellung.html) ist hinsichtlich Quellen leider nicht ergiebig.

116 Vgl. die Hinweise in Anm. 43. Einzubeziehen in die bild- und propagandageschichtliche Recherche wären auch die umfangreichen Ressourcen von Museen und Archiven in den Vereinigten Staaten, Großbritannien, Frankreich oder dem Beneluxraum, wo die Erinnerung und Aufarbeitung des Ersten Weltkrieges derzeit einen noch sehr viel größeren Stellenwert hat als in Deutschland oder Österreich (vgl. Bavendamm 2006; Dornik 2004: 95).

117 Vgl. http://www.historisches-lexikon-bayerns.de/artikel/artikel_44947; http://www.minderheiten.mkg-koeln.de/main/produktionstagebuch/die_recherche/3_01_02/3_01_02.htm.

118 Vgl. den Film zur Aufstellung der deutsch-französischen Brigade 1989 aus einer Internetausstellung zur Geschichte der Bundeswehr unter http://media.bwehr.de/Redaktionen/50jahre/mgfa/03/10_d414_300k.wmv.

119 Vgl. http://www.konrad-adenauer.de/; http://www.adenauerhaus.de/ oder auch http://helmutkohl.kas.de/index.php?menu_sel $=1 \&$ menu_sel $2=\& m e n u \_s e l 3=\& m e n u \_s e l 4=$.

120 Vgl. http://bismarckstiftung.de/index.php/startseite; http://www.dhm.de/lemo/html/biografien/BismarckOtto/index.html. - Für die österreichische Geschichte vgl. beispielsweise http://www.habsburger.net/.

121 Vgl. http://www.willy-brandt-luebeck.de/; http://www.willy-brandt.de/; http://www.ebertgedenkstaette.de/stiftung.html; http://www.fes.de/archiv/adsd_neu/inhalt/ebert.htm oder auch http://www.stiftung-heuss-haus.de/.

122 Vgl. http://www.goethehaus-frankfurt.de/; http://www.klassik-stiftung.de/; http://www.martinluther.de/; http://luther.chadwyck.co.uk/deutsch/html/frames/moreinfo/unsubscribed_g.htm (nur eingeschränkte Nutzung); http://www.zeno.org/Literatur/M/Luther,+Martin; http://www.bachhaus.de/; http://www.bach-leipzig.de/; http://www.beethoven-haus-bonn.de/.

${ }_{123}$ Vgl. nur http://www.koelner-dom.de/; http://www.aufbau-ffm.de/serie/Teil2/paulskirche.html; http://www.neuschwanstein.de/. 
Bräuchen und Ritualen, Festen und Feiern (jenseits von Karneval und Oktoberfest ${ }^{124}$ ), um Alltagskultur, Erziehungs- oder Mentalitätsmuster geht. ${ }^{125}$

Eine lebensweltliche Annäherung an die moderne deutsche Geschichte können allerdings auch Zeitzeugenerinnerungen, -berichte und -interviews, bringen. Trotz immer noch anklingender wissenschaftlicher Kritik sind sie inzwischen integraler Bestandteil von größeren Geschichtspräsentationen oder Onlineausstellungen. Dazu hat die Etablierung erfahrungsgeschichtlicher Perspektiven in der Geschichtsschreibung ebenso beigetragen wie der museumsdidaktische Trend zu Anschaulichkeit und Personalisierung und die besonders durch die NS-Geschichte geförderte Aufwertung persönlicher Zeugenschaft. Ausschlaggebend ist aber auch die allgemeine Popularität privaten Erinnerns und historisch kolorierter Familiengeschichten sowie das Bestreben der Massenmedien, historische Stoffe mit Erinnerungsberichten und Alltagsgeschichten einem breiteren Publikum nahe zu bringen. ${ }^{126}$

Aufgrund der audiovisuellen Möglichkeiten und guten Erreichbarkeit im WWW sind in letzter Zeit einige der zahlreichen Zeitzeugenarchive geöffnet ${ }^{127}$ und

$124 \mathrm{Vgl}$. z.B. http://www.karneval.de/geschichte.aspx; http://www.kk-museum.de/cms/front_con-
tent.php?client=1\&lang=1\&idcat=6; http://www.oktoberfest.de/de/article/Das+Oktoberfest/Ge-
schichte/Die+Geschichte+des+Oktoberfests/621/; http://www.bayerische-landesbibliothek-onli-
ne.de/oktoberfest. 125 Das Angebot ist hier disparat. Erziehungs- und schulgeschichtliche Quellen: http://geidigital.gei.de/cms/ („Die digitale Schulbuch-Bibliothek“, bisher Geschichtsschulbücher des Kaiserreichs erfasst); http://bbf.dipf.de/retro-digit0.htm/digitale-bbf/scripta-paedagogica-online (,Textarchiv zur Bildungsgeschichte des deutschsprachigen Raums“, bis 1945); http://www.bis.unioldenburg.de/retrodig/index.php (Digitalisierte historische Kinderbücher); http://en.childrenslibrary.org/ („International Children's Digital Library“, mit wenigen historischen deutschen Kinderbüchern); http://www.dhm.de/sammlungen/alltag3/spielzeug/bestand.html (Beispiele historischen Spielzeugs aus den Sammlungen des Deutschen Historischen Museums); http://www.kriegsspielzeug.org/ (kl. Präsentation zu Spielzeug im Kalten Krieg); http://www.deutschefotothek.de/ (mit Themenfilter „Schule“); http://www.schulmuseum.at/vsm/index2.htm (Virtuelles Schulmuseum Klagenfurt; stellvertretend für die verschiedenen Schulmuseen) sowie die Hinweise in Anm. 55 und 100. Vgl. auch http://www.bildpostkarten.uni-osnabrueck.de/index.php (Postkartensammlung mit Material zu zahlreichen alltagskulturellen Themen); http://www.liederlexikon.de/ (Texte und Bilder des Deutschen Volksliedarchivs); http://www.lwl.org/LWL/Kultur/VOKO/Archive_Bibliothek/Volkslied_Tonarchiv/ (mit Hörbeispielen); http://www.zeno.org/Kulturgeschichte (Benimmbücher des 18.-20. Jahrhunderts); http://gutenberg.spiegel.de/genre/fairy (Märchen); http://gutenberg.spiegel.de/genre/fable (Fabeln); https://www2.landesarchivbw.de/ofs21/olf/startbild.php?bestand $=2350$ (Bildsammlung eines süddeutschen Fotoateliers mit Aufnahmen von Festen und Feiern); http://www.siue.edu/COSTUMES/history.html (Publikation des 19. Jahrhunderts zur Geschichte der Kostüme); http://www.dilibri.de/nav/classification/55402 (Fastnachtszeitungen des 19. Jahrhunderts); eine ältere Sammlung zur Festkultur unter http://www.hab.de/bibliothek/wdb/festkultur/index.htm (Deutsche Drucke des 17. Jahrhunderts zur Festkultur des Barock).

126 Vgl. hierzu aus unterschiedlichen Blickwinkeln Assmann 2006: 85ff.; Fischer; Wirtz 2008; Handro 2009: 86ff.; Keilbach 22010; Köhr 2008; Lersch; Viehoff 2009: 99ff.; Welzer; Moller; Tschugnall 2002. 127 Mittlerweile gibt es in Deutschland eine Vielzahl von Zeitzeugenarchiven und „Erinnerungswerkstätten“, deren Bestände bisher nur punktuell ,übers Netz“ zugänglich sind. Zentrale und stilbildende Einrichtungen stammen aus den USA, so das an der Yale University untergebrachte, überwiegend englischsprachige Fortunoff Video Archive for Holocaust Testimonies (vgl. http://www.library.yale.edu/testimonies/excerpts/; eine Auswahl der knapp 4.500 Interviews 
Oral History-Projekte direkt fürs Netz konzipiert worden. Hier geht es darum, Betroffene nicht nur als Stichwortgeber für historische Darstellungen einzusetzen, sondern von persönlichen Erinnerungen und lebensgeschichtlichen Erzählungen auszugehen, um diese dann mit Dokumenten, Fotos und historischen Erläuterungen weiter aufzuschlüsseln. ${ }^{128}$

Das Internet bietet nicht bloß eine Plattform für erfahrungs- und lebensgeschichtliche Quellen; mit seinen interaktiven Möglichkeiten regt es die Nutzerinnen und Nutzer auch unmittelbar zur Quellenproduktion an, zum Schreiben von Erinnerungsgeschichten oder zum Entdecken historischer Zeugnisse im eigenen Umfeld. ${ }^{129}$ So gibt es neben privaten Websites, die Erfahrungsberichte zur DDRZeit sammeln, ${ }^{130}$ Projekte wie zeitzeugengeschichte.de, das Schülerinnen und Schüler zur „Erinnerungsarbeit“ mit Zeugen der NS-Zeit ermuntert,131 oder das von der Bundeszentrale für politische Bildung ins Leben gerufene „InternetArchiv“ „Wir waren so frei“, zu dem bereits zahlreiche Personen private Fotos, Filme und Impressionen beigesteuert haben. ${ }^{132}$ Große Resonanz findet auch das redaktionell stark formatierte, unverkennbar kommerziellen Interessen dienende Spiegel-Portal „einestages“, das Erinnerungen „kleiner Leute“ mit prominenten Biografien und großen historischen Szenarien zusammenschneidet. ${ }^{133}$

Auch andere, ältere erfahrungsgeschichtliche Quellen und Selbstzeugnisse sind gelegentlich im Internet zu entdecken. So finden sich dort verstreute Auszüge aus

des Archivs ist im „Ort der Information“ am Denkmal für die ermordeten Juden Europas in Berlin einsehbar) und das in den 1990er Jahren auf Initiative Steven Spielbergs eingerichtete Visual History Archive des Shoah Foundation Institute an der University of Southern California (vgl. http://dornsife.usc.edu/vhi/). Unter dessen ab 1994 geführten etwa 52.000 Interviews finden sich auch knapp 1.000 deutschsprachige; bis vor Kurzem waren die Interviewfilme jedoch nur an ausgewählten, lizenzierten Einrichtungen (etwa der Freien Universität Berlin [vollständig] oder dem Jüdischen Museum Berlin [deutschsprachige Interviews]) einsehbar, ein weitergehender Zugang über Internet ist offenbar angedacht. Vgl. http://www.zeugendershoah.de/, die Erläuterungen von Verena Lucia Nägel unter http://www.bpb.de/files/AEXU0E.pdf sowie Barricelli 2010; Meyer 2009c: 190f. und den Beitrag von Linde Apel in den Zeithistorischen Forschungen, http://www.zeithistorischeforschungen.de/site/40208872/default.aspx.

${ }_{128} \mathrm{Vgl}$. die Hinweise auf entsprechende Zeitzeugen- und Interviewprojekte in den Anm. 89, 91, 92, 99-101, 109, 110 sowie Leh 2009.

129 Zur Entwicklung des Web 2.0 in den letzten Jahren und den damit gegebenen Beteiligungsmöglichkeiten vgl. die Hinweise bei Meyer 2009a: 280ff.; Meyer 2009c; Pscheida 2010: 274ff., 291ff.; Spahn 2009: 302f. Erst an diesem Punkt - wenn historische Erinnerungen eigens für das World Wide Web aufgeschrieben oder private Materialen „im Netz“ zu bedeutsamen Quellen erklärt werden - wird das Internet ein ganz eigenständiges „Medium der Erinnerung“ Zit. n. Hein 2009a, 19.

${ }_{130}$ Vgl. die Hinweise in Anm. 101.

131 Vgl. http://zeitzeugengeschichte.de/ sowie Marzinka 2010; Meyer 2009c: 193.

132 Vgl. http:/ /www.wir-waren-so-frei.de/ („Momentaufnahmen 1989/1990“); Thema ist der Zusammenbruch der DDR und die „Wende-Zeit“".

133 Vgl. http://einestages.spiegel.de/page/Home.html sowie Meyer 2009a: 281ff.; Meyer 2009c: 198ff. - Ähnlich angelegt ist http://www.von-zeit-zu-zeit.de/ („Die Geschichtswerkstatt von Stuttgarter Zeitung und Stadtarchiv"); dazu auch die weiterführenden Hinweise in Lersch; Müller 2010. 
Tagebüchern ${ }^{134}$ ebenso wie (private) Editionen von Postkarten oder Feldpostbriefen aus dem Zweiten und Ersten Weltkrieg ${ }^{135}$. Solche „Ego-Dokumente“ werden zukünftig eine noch größere Rolle im Netz spielen, zumal es inzwischen auch Ansätze gibt, derartige Materialien gezielter zusammenzutragen und gebündelt im World Wide Web vorzustellen. ${ }^{136}$

\section{Schluss: Historische Quellen im Internet und Landeskunde}

Mit der Entwicklung des Internet zu einem Raum historischer Präsentation, Vermittlung, Auseinandersetzung und Erinnerung ist auch der Zugriff auf geschichtliche Ressourcen unzweifelhaft leichter geworden. Das World Wide Web mag ein „riesige[r] Wissensspeicher“ (Pscheida 2010: 283f.) geworden sein. Allerdings ist es (noch) längst kein „,virtuelles Archiv“ oder ein ,virtueller Lesesaal“, in dem man einen einfachen, systematischen und umfassenden Zugriff auf historische Quellen, Bilder, Töne und Dokumente bekommt. Das Netz liefert zusätzliches Material für die didaktische Arbeit, um besser auf die jeweiligen Geschichtsbilder der Lernenden einzugehen, Lerneinheiten speziell audiovisuell anzureichern und die zum Teil problematischen Darstellungen ${ }^{137}$ älterer Lehrwerke zu umgehen. Dies macht jedoch auch einige Suchbewegungen erforderlich sowie eine kritische

\footnotetext{
134 Vgl. http://www.tagebucharchiv.de/texte/bilderserien.htm bzw. http://www.tagebucharchiv.de/texte/leseecke.htm (Auszüge aus den Beständen des Deutschen Tagebucharchivs); http://zeitstimmen.de/ („Tagebuchliteratur aus Brandenburg"); http://grossmann.weebly.com/ (Tagebuch eines Lehrers und Soldaten aus dem Zweiten Weltkrieg); http://www.buehler-hd.de/landeskunde/rhein/geschichte/zeitgeschichte/wk1/tagebuch.htm (Tagebuch eines badischen Soldaten im Ersten Weltkrieg). Für frühere Epochen: http://www.mdsz.thulb.uni-jena.de/ („Mitteldeutsche Selbstzeugnisse der Zeit des Dreißigjährigen Krieges").

135 Vgl. v.a. http:/ /www.museumsstiftung.de/feldpost (Online-Sammlung der Museumsstiftung Post und Telekommunikation mit 1.400 Feldpostbriefen zum Zweiten Weltkrieg) sowie http://www.feldpost-archiv.de/; http://www.digada.de/wk1/uebersichtwk1.htm (Feldpostbriefe Darmstädter Soldaten aus dem Ersten Weltkrieg); http://www.dradio.de/dlf/sendungen/feldpost/ (sendebegleitende Materialien zum Ersten Weltkrieg); als Beispiele für die zahlreichen privaten Initiativen http://www.feldpostbriefe.de/index2.html; http://www.clooney.at/feldpost/; http://www.peter-engelhardt.com/; http://klee-klaus.business.t-online.de/vermisst.htm; http://www.hier-ist-es-schoen.de/HP/Sadelkow-fr.htm (Briefe und Postkarten von Soldaten zum Ersten Weltkrieg) sowie http://www.32postkarten.com/index_D.html (Postkarten einer deutsch-jüdischen Familie aus den 1940er Jahren). Vgl. auch http://www.ub.uni-heidelberg.de/helios/digi/feldzeitungen.html (digitalisierte „Schützengrabenzeitungen“); für frühere Epochen http://www.digam.net/?str=122 (Feldpostbriefe 1866, 1870/71).

136 So soll das Projekt Europeana 1914-1918 bis 2014 hunderttausende digitalisierte Quellen zum Ersten Weltkrieg europaweit verfügbar und dabei auch bisher nicht greifbare, zumal alltagsgeschichtliche Quellen der Öffentlichkeit zugänglich machen. Ausdrücklich werden auch Privatpersonen aufgefordert, ihre Erinnerungsstücke zur Verfügung zu stellen. Vgl. http://group.europeana.eu/web/guest/details-europeana1914-1918/. Einen ähnlichen Ansatz verfolgt das HOPE-Projekt („Heritage of People's Europe“), das v.a. sozialgeschichtliche Quellen bündelt; vgl. http://www.peoplesheritage.eu/.

137 Besonders deutliche und differenzierte Kritik haben Darstellungen zur neueren deutschen und NS-Geschichte erfahren; vgl. Ghobeyshi 2002; Koreik 1995: 142ff.; Thimme 1996: 97ff.; Warmbold; Koeppel; Simon-Pelanda 1994).
} 
Reflexion der im Internet präsentierten Materialien, ihres Verwendungszusammenhangs und ihrer didaktischen Brauchbarkeit (Rösler 2010: 1207f.). Zwar gibt es einige Internetangebote, die unterschiedliche Quellen, verschiedene historische Perspektiven und breite, differenzierte Hintergrundinformationen liefern; kompakte, direkt nutzbare Lehr- und Lernumgebungen sind aber immer noch vergleichsweise selten. Insofern wird man bei vielen Themen noch auf ,analoge“ Lehrmittel oder DVDs zurückgreifen, die nicht bloß Quellenmaterial liefern, sondern intensivere redaktionelle Bearbeitung garantieren und produktive Didaktisierungen bieten. ${ }^{138}$

Mit Blick auf das Fach Deutsch als Fremdsprache und die Ansprüche einer historisch basierten Landeskunde lässt sich folgendes vorsichtiges und natürlich vorübergehendes Fazit ziehen:

- Im Internet finden sich etliche Hilfsmittel und Materialien für die historische Faktenvermittlung, auch für die kritische Reflexion der selbst bei interessierten Lernern verbreiteten Mythen, geschichtspolitischen Legenden oder unscharfen Geschichtsbilder (vgl. Borries 2000; Illy 1999; Koreik 1995: 82ff.; Thimme 1996: 79ff.; Warmbold; Koeppel; Simon-Pelanda 1994). Das gilt besonders, wenn man neben historischen Quellen die „quellenbegleitenden“ Texte und Materialien einbezieht.

- Differenzierter muss geurteilt werden, wenn man die „kommunikative“ und „interkulturelle“ Ausrichtung der Landeskunde, Kommunikationssicherheit, Handlungskompetenz, Reflexion und „Fremdverstehen“ betont. Eine solche Landeskunde bedarf anderer Materialien und historischer Quellen als eine einfache „Realien-“ oder „Staatsbürgerkunde“. Solche „anderen“, sozial-, kultur- und mentalitätsgeschichtlichen Quellen lassen sich im Internet durchaus finden, sind jedoch nicht so leicht greifbar. Auch hinsichtlich der Auseinandersetzung mit historisch bedingten Stereotypen und Vorurteilen mangelt es m.E. noch an Plattformen, die Materialien sammeln, bündeln oder schnellen Zugriff auf „sprechende“, anschauliche Quellen erlauben.

- Die starke Präsenz der NS-Zeit und der DDR-Geschichte im Internet entspricht der Entwicklung der deutschen Erinnerungskultur in den letzten Jahrzehnten. Insofern vermitteln Quellen und Materialien zu diesen Epochen nicht allein historisches Wissen, sondern Orientierung in der deutschen Gegenwartsgesellschaft, die in öffentlichen Debatten, politischen Entscheidungen, Familiengeschichten und Alltagskommunikation immer wieder auf NS-Diktatur und „SEDRegime" Bezug nimmt.

138 Vgl. den - älteren - Überblick bei Oswalt 2006 und die entsprechenden Beiträge in Danker; Schwabe 2008a; zu den zahlreichen DVD-Editionen des Deutschen Historischen Museums Jahrbuch 2007: 47ff. - Als gelungenes Beispiel für eine quellenorientierte Auseinandersetzung mit „Fixpunkten“ deutscher Geschichte: Schmidt; Schmidt 2007; als Beispiel für die Auseinandersetzung mit historisch gewachsenen Mentalitätsmustern, Stereotypen und Vorurteilen: Behal-Thomsen; Lundquist-Mog; Mog 1993. 
- Andere geschichtliche Prägungen der deutschen Gesellschaft scheinen über Internetressourcen und WWW-Quellen weniger dicht zu erfassen zu sein, etwa Umweltschutz und Naturromantik, der deutsche Weg der Sozialstaatlichkeit, Ordnungsvorstellungen, Obrigkeits- und Staatsverständnis. Aspekte wie Frauenunterdrückung und -emanzipation, der Wertewandel und die kulturelle Liberalisierung seit den 1960er Jahren sowie Realitäten von „Deutschland als Einwanderungsland“ haben zuletzt aber auch im Netz stärkere Beachtung gefunden. Die Veränderungen des Geschichtsbewusstseins und die Dynamik des World Wide Web machen es wahrscheinlich, dass es hierzu bald weitere brauchbare Internetangebote geben wird.

- „Alltag und Erfahrung“ als Perspektiven historischer Landeskunde lassen sich im Netz derzeit am ehesten über Zeitzeugenberichte und lebensgeschichtliche Interviews einfangen. Wenngleich solche Quellen besonderer methodischer Reflexion bedürfen, in der Unterrichtspraxis bisher nicht im Zentrum stehen (vgl. Illy 1999: Abschn. 3.1.5) und von der sprachlichen Textur gerade Fremdsprachenlernern Schwierigkeiten bereiten können, stellen sie aktuell doch eines der wichtigsten Angebote des Internet an die Landeskunde dar.

\section{Literaturangaben}

Alavi, Bettina (2008): Zeitgeschichte virtuell: Zur Didaktisierung zeitgeschichtlicher Webangebote am Beispiel der Website www.chronik-der-mauer.de. In: Barricelli; Hornig (Hrsg.) (2008), 109-120.

Alavi, Bettina (Hrsg.) (2010): Historisches Lernen im virtuellen Medium. Heidelberg: Mattes.

Altrichter, Helmut; Antipow, Lilia (2009): Geschichte als Politik. Quelleneditionen „100(0) Schlüsseldokumente zur russischen und sowjetischen Geschichte (1917-1991)“ und „100(0) Schlüsseldokumente zur deutschen Geschichte im 20. Jahrhundert“ im Internet. In: Neuhaus, Helmut (Hrsg.) (2009): Erlanger Editionen. Grundlagenforschung durch Quelleneditionen: Berichte und Studien. Erlangen, Jena: Palm \& Enke, 471-493.

Arnold, Jörg; Süß, Dietmar; Thießen, Malte (Hrsg.) (2009): Luftkrieg. Erinnerungen in Deutschland und Europa. Göttingen: Wallstein.

Assmann, Aleida (2004): Zur Mediengeschichte des kulturellen Gedächtnisses. In: Erll, Astrid; Nünning, Ansgar (Hrsg.) (2004): Medien des kollektiven Gedächtnisses. Konstruktivität - Historizität - Kulturspezifität. Berlin, New York: de Gruyter, 4560.

Assmann, Aleida (2006): Der lange Schatten der Vergangenheit. Erinnerungskultur und Geschichtspolitik. München: Beck. 
Barricelli, Michele (2010): Kommemorativ oder kollaborativ? Historisches Lernen mithilfe digitaler Zeitzeugenarchive (am Beispiel des Visual History Archive). In: Alavi (Hrsg.) (2010), 13-30.

Barricelli, Michele; Hornig, Julia (Hrsg.) (2008): Aufklärung, Bildung, „Histotainment“? Zeitgeschichte in Unterricht und Gesellschaft heute. Frankfurt/Main u.a.: Lang.

Bausch, Karl-Richard; Christ, Herbert; Krumm, Hans-Jürgen (Hrsg.) (42003): Handbuch Fremdsprachenunterricht. Tübingen, Basel: A. Francke.

Bavendamm, Gundula (2006): Der Erste Weltkrieg im Internet. In: Groß, Gerhard P. (Hrsg.) (2006): Die vergessene Front. Der Osten 1914/15. Ereignis, Wirkung, Nachwirkung. Paderborn u.a.: Schöningh, 373-391.

Behal-Thomsen, Heinke; Lundquist-Mog, Angelika; Mog, Paul (1993): Typisch deutsch? Arbeitsbuch zu Aspekten deutscher Mentalität. Berlin u.a.: Langenscheidt.

Behrens, Heidi; Ciupke, Paul; Reichling, Norbert (Hrsg.) (2009): Lernfeld DDRGeschichte. Ein Handbuch für die politische Jugend- und Erwachsenenbildung. Schwalbach/Ts.: Wochenschau 2009.

Beier, Rosmarie (2000): Geschichte, Erinnerung und Neue Medien. Überlegungen am Beispiel des Holocaust. In: Beier, Rosmarie (Hrsg.) (2000): Geschichtskultur in der Zweiten Moderne. Frankfurt/Main, New York: Campus, 299-323.

Beier-de Haan, Rosmarie (Hrsg.) (2005): Erinnerte Geschichte - Inszenierte Geschichte. Ausstellungen und Museen in der Zweiten Moderne. Frankfurt/Main: Suhrkamp.

Beine, Jürgen (2009): Wikis als Herausforderung für die Geschichtswissenschaften. In: Histnet Wiki (http://wiki.histnet.ch/Wikis_als_Herausforderung_$\mathrm{f} \% \mathrm{C} 3 \% \mathrm{BCr}$ _die_Geschichtswissenschaft).

Bösch, Frank (2006): Holokaust mit „K““. Audiovisuelle Narrative in neueren Fernsehdokumentationen. In: Paul, Gerhard (Hrsg.) (2006): Visual history. Ein Studienbuch. Göttingen: Vandenhoeck \& Ruprecht, 317-332.

Bösch, Frank (2007): Film, NS-Vergangenheit und Geschichtswissenschaft. Von „Holocaust“ zu „Der Untergang“. In: Vierteljabrshefte für Zeitgeschichte 55, 1-32.

Bösch, Frank (2011): Mediengeschichte. Vom asiatischen Buchdruck zum Fernsehen. Frankfurt/Main, New York: Campus.

Bösch, Frank; Goschler, Constantin (Hrsg.) (2009): Public History. Öffentliche Darstellungen des Nationalsozialismus jenseits der Geschichtswissenschaft. Frankfurt/Main, New York: Campus.

Borries, Bodo von (2000): Nationale Geschichtskulturen und jugendliche Geschichtsvorstellungen im europäischen Vergleich. In: Mütter, Bernd; Schönemann, Bernd; Uffelmann, Uwe (Hrsg.) (2000): Geschichtskultur. TheorieEmpirie - Pragmatik. Weinheim: Deutscher Studien Verlag, 307-335. 
Brautmeier, Jürgen et al. (Hrsg.) (2010): Heimat Nordrhein-Westfalen. Identitäten und Regionalität im $W$ andel. Essen: Klartext.

Burckhardt, Daniel; Hohls, Rüdiger; Prinz, Claudia (Hrsg.) (2007): Geschichte im Netz: Praxis, Chancen, Visionen. Beiträge der Tagung .hist 2006. Berlin: Selbstverlag (http://edoc.hu-berlin.de/histfor/10_I/PDF/HistFor_2007-10-I.pdf und http:// edoc.hu-berlin.de/histfor/10_II/PDF/HistFor_2007-10-II.pdf).

Burckhardt, Daniel; Hohls, Rüdiger; Ziegeldorf, Vera (Hrsg.) (2005): Geschichte und Neue Medien in Forschung, Archiven, Bibliotheken und Museen. Tagungsband .hist 2003. Berlin: Selbstverlag (http://edoc.hu-berlin.de/e_histfor/7_I/PDF/HistFor_72005-I.pdf und http://edoc.hu-berlin.de/e_histfor/7_II/PDF/HistFor_72005-II.pdf).

Cornelißen, Christoph (2008): Internet und Geschichtswissenschaft. Anmerkungen aus der Praxis von historischer Lehre und Forschung. In: Hartung, Olaf; Köhr, Katja (Hrsg.) (2008): Geschichte und Geschichtsvermittlung. Festschrift für Karl Heinrich Pobl. Bielefeld: Verlag für Regionalgeschichte.

Cyron, Marcus (2009): User generated history. Wikipedia als digitales Geschichtsschreibungsprojekt. In: Hardtwig; Schug (Hrsg.) (2009), 256-263.

Czmiel, Alexander et al. (2005): Retrospektive Digitalisierung von Bibliotheksbeständen. Evaluierungsbericht über einen Förderschwerpunkt der DFG. Köln: Universität zu Köln (http://www.deutsche-digitale-bibliothek.de/pdf/retro_digitalisierung_eval_050406.pdf).

Dalmas, Martine (2010): Deutsch in Frankreich. In: Krumm et al. (Hrsg.) (2010), 1658-1664.

Danker, Uwe; Schwabe, Astrid (Hrsg.) (2008a): Historisches Lernen im Internet. Geschichtsdidaktike und Neue Medien. Schwalbach/Ts.: Wochenschau.

Danker, Uwe; Schwabe, Astrid (2008b): Normative fachdidaktische Anforderungen an virtuelle Geschichtspräsentationen. Möglichkeiten und Grenzen der Umsetzung am Projektbeispiel eines „Virtuellen Museums“. In: Danker; Schwabe (Hrsg.) (2008a), 60-89.

Dornik, Wolfram (2004): Erinnerungskulturen im Cyberspace. Eine Bestandsaufnahme österreichischer Websites zu Nationalsozialismus und Holocaust. Berlin: Trafo.

Dornik, Wolfram (2010): Internet: Maschine des Vergessens oder globaler Gedächtnisspeicher? Der Holocaust in den digitalen Erinnerungskulturen zwischen 1990 und 2010. In: Paul, Gerhard; Schoßig, Bernhard (Hrsg.): Öffentliche Erinnerung und Medialisierung des Nationalsozialismus. Eine Bilanz der let_ten dreißig Jahre. Göttingen: Wallstein, 79-97.

Echternkamp, Jörg; Martens, Stefan (Hrsg.) (2007): Der Zweite Weltkrieg in Europa. Erfabrungen und Erinnerungen. Paderborn u.a.: Schöningh. 
Eckel, Jan; Moisel, Claudia (Hrsg.) (2008): Universalisierung des Holocaust? Erinnerungskultur und Geschichtspolitik in internationaler Perspektive. Göttingen: Wallstein.

Eder, Franz X. et al. (2006): Geschichte Online. Einführung in das wissenschaftliche Arbeiten. Wien u.a.: Böhlau.

Eder, Franz X.; Fuchs, Eduard (2005): Lernmodelle und Neue Medien. Historisches Lehren und Lernen am Beispiel „Geschichte Online“ (GO). In: Epple; Haber (Hrsg.) (2005), 163-181.

Enderle, Wilfried (2002): Der Historiker, die Spreu und der Weizen. Zur Qualität und Evaluierung geschichtswissenschaftlicher Internetressourcen. In: Haber; Koller; Ritter (Hrsg.) (2002), 49-63.

Epple, Angelika; Haber, Peter (Hrsg.) (2005): Vom Nutzen und Nachteil des Internet für die historische Erkenntnis. Version 1.0. Zürich: Chronos.

Erpel, Simone (2010): Hitler entdämonisiert. Die mediale Präsenz des Diktators nach 1945 in Presse und Internet. In: Thamer, Hans-Ulrich; Erpel, Simone (Hrsg.): Hitler und die Deutschen. Volkesgemeinschaft und Verbrechen. Dresden: Sandstein, 154-160.

Euler, Ellen (2011): Das kulturelle Gedächtnis im Zeichen digitaler und vernetzter Medien und sein Recht. Status Quo der rechtlichen, insbesondere urheberrechtlichen Rabmenbedingungen von Bestandsaufbau, Bestandserhaltung und kommunikativer sowie kommerzieller Bestandsvermittlung kultureller Äußerungen im Zeitalter digitaler und vernetżter Medien durch Bibliotheken, Archive und Museen in Deutschland und Regelungsalternativen. Bad Honnef: Bock+Herchen.

Fischer, Thomas; Wirtz, Rainer (Hrsg.) (2008): Alles authentisch? Popularisierung der Geschichte im Fernsehen. Konstanz: UVK.

Fischer, Torben; Lorenz, Matthias N. (2007): Lexikon der „Vergangenheitsbewältigung “ in Deutschland. Debatten- und Diskursgeschichte des Nationalsozialismus nach 1945.

Bielefeld: transcript.

Gasteiner, Martin; Haber, Peter (Hrsg.) (2010): Digitale Arbeitstechniken für Geistesund Kulturwissenschaften. Wien u.a.: Böhlau.

Geschichte in Wissenschaft und Unterricht. Seelze: Friedrich Verlag, Jg. 49ff. (1998ff.).

Geschichte lernen. Seelze: Friedrich Verlag, Jg. 12ff. (1999ff.).

Ghobeyshi, Silke (2002): Nationalsozialismus und Schoah als landeskundliche Themen im DaF-Unterricht. Frankfurt/Main u.a.: Lang.

Görtemaker, Manfred (2004): Geschichte der Bundesrepublik Deutschland. Von der Gründung bis zur Gegenwart. Frankfurt/Main: Fischer. 
Grosch, Waldemar (2002): Geschichte im Internet. Tipps, Tricks und Adressen. Schwalbach/Ts.: Wochenschau.

Grosch, Waldemar (2008): Das Internet als Raum historischen Lernens - eine Bestandsaufnahme. In: Danker; Schwabe (Hrsg.) (2008a), 13-35.

Große Kracht, Klaus (2005): Die zankende Zunft. Historische Kontroversen in Deutschland nach 1945. Göttingen: Vandenhoeck \& Ruprecht.

Gruner, Martin (2009): Quellen online: Prozessdokumente im Internet. In: Finger, Jürgen; Keller, Sven; Wirsching, Andreas (Hrsg.) (2009): Vom Recht zur Geschichte. Akten aus NS-Prozessen als Quellen der Zeitgeschichte. Göttingen: Vandenhoeck \& Ruprecht, 254-259.

Haber, Peter (2005): „Google-Syndrom“. Phantasmagorie des historischen Allwissens im World Wide Web. In: Epple; Haber (Hrsg.) (2005), 73-89.

Haber, Peter (2009): digital.past. Geschichtswissenschaft im digitalen Zeitalter. [Preprint im Internet.] Basel: Selbstverlag (demnächst: München: Oldenbourg 2011).

Haber, Peter; Koller, Christophe; Ritter, Gerold (Hrsg.) (2002): Geschichte und Internet. „Raumlose Orte - Geschichtslose Zeit“. Zürich: Chronos.

Handro, Saskia (2009): Mutationen. Geschichte im kommerziellen Fernsehen. In: Oswalt, Vadim; Pandel, Hans-Jürgen (Hrsg.) (2009): Geschichtskultur. Die Anwesenheit von Vergangenheit in der Gegenwart. Schwalbach/Ts.: Wochenschau, 75-97.

Hardtwig, Wolfgang; Schug, Alexander (Hrsg.) (2009): History sells! Stuttgart: Steiner.

Hartung, Olaf (Hrsg.) (2006): Museum und Geschichtskultur. Ästhetik - Politik Wissenschaft. Bielefeld: Verlag für Regionalgeschichte.

Hein, Dörte (2005): Mediale Darstellungen des Holocaust. Zum World Wide Web und zu seiner Disposition als Gedächtnismedium. In: Jabrbuch für Kommunikationsgeschichte 7, 176-196.

Hein, Dörte (2009a): Erinnerungskulturen online. Angebote, Kommunikatoren und Nutzer von Websites zu Nationalsozialismus und Holocaust. Konstanz: UVK.

Hein, Dörte (2009b): „Seriöse Information“ oder „schöne Bilder“? Kommemorative Kommunikation aus der Perspektive der Anbieter. In: Meyer, Erik (Hrsg.) (2009b), 145-173.

Hering, Rainer (Hrsg.) (2006): Forschung in der digitalen Welt. Sicherung, Erschließung und Aufbereitung von Wissensbeständen. Tagung des Staatsarchivs Hamburg und des Zentrums „Geisteswissenschaften in der digitalen Welt“ an der Universität Hamburg am 10. und 11. April 2006. Hamburg: Hamburg University Press. 
Hodel, Jan (2007): Historische Online-Kompetenz. Informations- und Kommunikationstechnologie in den Geschichtswissenschaften. In: Pöppinghege, Rainer (Hrsg.) (2007): Geschichte lehren an der Hochschule. Bestandsaufnahme, methodische Ansätže, Perspektiven. Schwalbach/Ts.: Wochenschau, 194-210.

Hörisch, Jochen (2004): Eine Geschichte der Medien. Von der Oblate zum Internet. Frankfurt/Main: Suhrkamp.

Huneke, Hans-Werner; Steinig, Wolfgang (52010): Deutsch als Fremdsprache. Eine Einfübrung. Berlin: Schmidt.

Illy, Regine (1999): Nationalsozialismus als Thema im Deutsch als FremdspracheUnterricht. In: Forscbungs- und Arbeitsstelle „Erriehung nach/über Auschwitz", Downloadbereich (http://www.fasena.de/download/daf/Illy.pdf).

Jahrbuch (2007): Jabrbuch der historischen Forschung in der Bundesrepublik Deutschland. Berichtsjabr 2006. München: Oldenbourg.

Jakobi, Franz-Josef (2000): Archive und Geschichtskultur. In: Mütter, Bernd; Schönemann, Bernd; Uffelmann, Uwe (Hrsg.) (2000): Geschichtskultur. Theorie Empirie - Pragmatik. Weinheim: Deutscher Studien Verlag, 130-141.

Jarausch, Konrad H.; Sabrow, Martin (Hrsg.) (2002): Die historische Meistererzählung. Deutungslinien der deutschen Nationalgeschichte nach 1945. Göttingen: Vandenhoeck \& Ruprecht.

Jenks, Stuart; Marra, Stephanie (Hrsg.) (2001): Internet-Handbuch Geschichte. Böhlau: Köln u.a.

Kansteiner, Wulf (2006): In Pursuit of German Memory. History, Television, and Politics after Auschwit: Athens: Ohio University Press.

Keilbach, Judith (2010): Geschichtsbilder und Zeitzengen. Zur Darstellung des Nationalsozialismus im bundesdeutschen Fernsehen. Münster: Lit.

Köhr, Katja (2008): Die vielen Gesichter des Holocaust - Individualisierung als Konzept musealer Geschichtsvermittlung. In: Handro, Saskia; Schönemann, Bernd (Hrsg.) (2008): Orte historischen Lernens. Berlin, Münster: Lit, 165-177.

Kolpatzik, Andrea: „Die Deutschen“ erobern die Schule! Grenzen und Chancen historischen Lernens mit kommerziellen Geschichtsportalen. In: Alavi (Hrsg.) (2010), 201-216.

Koreik, Uwe (1995): Deutschlandstudien und deutsche Geschichte. Die deutsche Geschichte im Rahmen des Landeskundeunterrichts für Deutsch als Fremdsprache. Baltmannsweiler: Schneider.

Koreik, Uwe (2010): Landeskundliche Gegenstände: Geschichte. In: Krumm et al. (Hrsg.) (2010), 1478-1483. 
Korte, Barbara; Paletschek, Sylvia (Hrsg.) (2009): History Goes Pop. Zur Repräsentation von Geschichte in populären Medien und Genres. Bielefeld: transcript.

Kröll, Ulrich (2010): Zeitgeschichte in digitalen Medien. In: Popp et al. (Hrsg.) (2010), 149-170.

Krumm, Hans-Jürgen et al. (Hrsg.) (2010): Deutsch als Fremd- und Zweitsprache. Ein internationales Handbuch. Berlin, New York: de Gruyter.

Kruse, Meike (2008): Das Archiv der Hansestadt Lübeck online. Konzept einer Internetund Intranetpräsentation. Saarbrücken: VDM.

Kühberger, Christoph; Sedmak, Clemens (Hrsg.) (2009): Europäische Geschichtskultur - Europäische Geschichtspolitik. Vom Erfinden, Entdecken, Erarbeiten der Bedeutung von Erinnerung und Geschichte für das Verständnis und Selbstverständnis Europas. Innsbruck u.a.: Studien Verlag.

Kümper, Hiram (2010): Zeitgeschichte und Wikipedia: von der Wissens(ver)schleuder(ung) zum Forschungsfeld. In: Popp et al. (Hrsg.) (2010), 283-296.

Kundrus, Birthe (Hrsg.) (2003): Phantasiereiche. Zur Kulturgeschichte des deutschen Kolonialismus. Frankfurt/Main, New York: Campus.

Laak, Dirk van (2005): Über alles in der Welt. Deutscher Imperialismus im 19. und 20. Jabrhundert. München: Beck.

Lange, Dirk (Hrsg.) (2008): Migration und Bürgerbewusstsein. Perspektiven Politischer Bildung in Europa. Wiesbaden: VS Verlag für Sozialwissenschaften.

Leggewie, Claus; Lang, Anne (2011): Der Kampf um die europäische Erinnerung. Ein Schlachtfeld wird besichtigt. München: Beck.

Leh, Almut (2009): Zeitzeugen online. Archive und andere Web-Angebote. In: Bios 22, 268-282.

Lehmann, Kai; Schetsche, Michael (Hrsg.) (2005): Die Google-Gesellschaft. Vom digitalen $W$ andel des Wissens. Bielefeld: transcript.

Lersch, Edgar; Müller, Peter (Hrsg.) (2010): Archive und Medien. Vorträge des 69. Südwestdeutschen Archivtags am 20. Juni 2009 in Münsingen. Stuttgart: Kohlhammer.

Lersch, Edgar; Viehoff, Reinhold (2009): Folgenlose Unterhaltung oder kunstvoller Wissenstransfer? Geschichtsfernsehen. In: Hardtwig; Schug (Hrsg.) (2009), 91-105.

Levy, Daniel; Sznaider, Natan (2001): Erinnerung im globalen Zeitalter. Der Holocaust. Frankfurt/Main: Suhrkamp.

Lorenz, Maren (2009a): Repräsentation von Geschichte in Wikipedia oder: Die Sehnsucht nach Beständigkeit im Unbeständigen. In: Korte; Paletschek (Hrsg.) (2009), 289-312. 
Lorenz, Maren (2009b): Wikipedia als „Wissensspeicher“ der Menschheit - genial, gefährlich oder banal? In: Meyer, Erik (Hrsg.) (2009b), 207-236.

Machill, Marcel; Beiler, Markus (Hrsg.) (2007): Die Macht der Suchmaschinen. Köln: von Halem.

Maier, Gerald (2007): Kulturgut aus Archiven, Bibliotheken und Museen im Internet. In: Buchholz, Matthias et al. (Hrsg.) (2007): Samisdat in Mitteleuropa. Prozess - Archiv - Erinnerung. Dresden: w.e.b. Universitätsverlag, 127-139.

Marra, Stephanie (2005): Geschichtsangebote im Internet: Populäre Rezeption und wissenschaftliche Vermittlung. In: Epple; Haber (Hrsg.) (2005), 131-138.

Marzinka, Birgit: Möglichkeiten von Bildungsportalen am Beispiel von zeitzeugengeschichte.de. In: Alavi (Hrsg.) (2010), 217-237.

Meyer, Erik (2009a): Problematische Popularität? Erinnerungskultur, Medienwandel und Aufmerksamkeitsökonomie. In: Korte; Paletschek (Hrsg.) (2009), 267-287.

Meyer, Erik (Hrsg.) (2009b): Erinnerungskultur 2.0. Kommemorative Kommunikation in digitalen Medien. Frankfurt/Main, New York: Campus.

Meyer, Erik (2009c): Erinnerungskultur 2.0? Zur Transformation kommemorativer Kommunikation in digitalen, interaktiven Medien. In: Meyer, Erik (Hrsg.) (2009b), 175-206.

Motte, Jan; Ohliger, Rainer (Hrsg.) (2004): Geschichte und Gedächtnis in der Einwanderungsgesellschaft. Migration zwischen historischer Rekonstruktion und Erinnerungspolitik. Essen: Klartext.

Näpel, Oliver (2003): Historisches Lernen durch „Dokutainment“? Ein geschichtsdidaktischer Aufriss. Chancen und Grenzen einer neuen Ästhetik populärer Geschichtsdokumentationen, analysiert am Beispiel der Sendereihen Guido Knopps. In: Zeitschrift für Geschichtsdidaktik 2, 213-244.

Näpel, Oliver (2008): Historisches Lernen im Internet. Legitimation, Anspruch und Wirklichkeit geschichtsdidaktischer Normative für Geschichtsangebote im Cyberspace. In: Danker; Schwabe (Hrsg.) (2008a), 90-107.

Nissen, Mogens R. (2008): „Virtuelles Museum“: Okkupation und Widerstand im Internet. Ein Projektbericht. In: Bohn, Robert; Cornelißen, Christoph; Lammers, Karl Christian (Hrsg.) (2008): Vergangenheitspolitik und Erinnerungskulturen im Schatten des Zweiten Weltkriegs. Deutschland und Skandinavien seit 1945. Essen: Klartext, 199-213.

Nonn, Christoph (2007): Was ist und zu welchem Zweck betreibt man Landeszeitgeschichte? Zu Problemen und Perspektiven einer Landesgeschichte der Moderne. In: Geschichte im Westen 21, 155-171. 
Oswalt, Vadim (2006): Elektronische Speichermedien. Teil I und II. In: Geschichte in Wissenschaft und Unterricht 57, 604-618, 677-690.

Paul, Gerhard (2010): Holocaust - Vom Beschweigen zur Medialisierung. Über Veränderungen im Umgang mit Holocaust und Nationalsozialismus in der Mediengesellschaft. In: Paul, Gerhard; Schoßig, Bernhard (Hrsg.): Öffentliche Erinnerung und Medialisierung des Nationalsozialismus. Eine Bilanz der letzten dreißig Jahre. Göttingen: Wallstein, 15-38.

Pfanzelter, Eva (2010): Von der Quellenkritik zum kritischen Umgang mit digitalen Ressourcen. In: Gasteiner; Haber (Hrsg.) (2010), 39-49.

Pieper, Katrin (2010): Resonanzräume. Das Museum im Forschungsfeld Erinnerungskultur. In: Baur, Joachim (Hrsg.) (2010): Museumsanalyse. Methoden und Konturen eines neuen Forschungsfeldes. Bielefeld: transcript, 187-212.

Pöppinghege, Rainer (2010): „die echt konkrete seite“ - LeMO als Lernort der Zeitgeschichte? In: Popp et al. (Hrsg.) (2010), 297-306.

Popp, Susanne et al. (Hrsg.) (2010): Zeitgeschichte - Medien - Historische Bildung. Göttingen: V\&R unipress.

Pscheida, Daniela (2010): Das Wikipedia-Universum. Wie das Internet unsere Wissenskultur verändert. Bielefeld: transcript.

Praxis Geschichte. Braunschweig: Westermann, Jg. 13ff. (2000ff.).

Reichel, Peter (2007): Vergangenheitsbewältigung in Deutschland. Die Auseinandersetzung mit der NS-Diktatur von 1945 bis heute. München: Beck.

Reichel, Peter; Schmid, Harald; Steinbach, Peter (Hrsg.) (2009): Der Nationalsozialismus. Die zweite Geschichte. Überwindung, Deutung, Erinnerung. München: Beck.

Rettinger, Elmar; Schrade, Torsten (2006): Forschen - Vermitteln - Mitmachen. Landesgeschichte im Zeitalter von Web 2.0 und Social Software. In: Blätter für deutsche Landesgeschichte 141/142 (2005/2006), 641-661.

Rösler, Dietmar (2010): Die Funktion von Medien im Deutsch als Fremd- und Deutsch als Zweitsprache-Unterricht. In: Krumm et al. (Hrsg.) (2010), 11991214.

Sabrow, Martin; Jessen, Ralph; Große Kracht, Klaus (Hrsg.) (2003): Zeitgeschichte als Streitgeschichte. Große Kontroversen seit 1945. München: Beck.

Sabrow, Martin (Hrsg.) (2009): Erinnerungsorte der DDR. München: Beck.

Sabrow, Martin (Hrsg.) (2010): Bewältigte Diktaturvergangenheit? 20 Jahre DDRAufarbeitung. Leipzig: AVA. 
Sahle, Patrick (2001): Digitale Editionstechniken und historische Quellen. In: Jenks; Marra (Hrsg.) (2001), 153-166.

Sahle, Patrick (2009): Das Archiv als virtualisierte Forschungsumgebung? In: Histnet Wiki (http://wiki.histnet.ch/Das_Archiv_als_virtualisierte_Forschungsumgebung $\% 3 \mathrm{~F})$.

Schildt, Axel; Siegfried, Detlef (2009): Deutsche Kulturgeschichte. Die Bundesrepublik. 1945 bis zur Gegenwart. München: Hanser.

Schlögl, Daniel (2006): Geschichte und länderbezogene Informationen im Internet. In: Syré, Ludger; Wiesenmüller, Heidrun (Hrsg.) (2006): Die Regionalbibliographie im digitalen Zeitalter. Deutschland und seine Nachbarländer. Frankfurt/Main: Klostermann, 151-164.

Schmale, Wolfgang (2010): Digitale Geschichtswissenschaft. Wien u.a.: Böhlau.

Schmale, Wolfgang et al. (2007): E-Learning Geschichte. Wien u.a.: Böhlau.

Schmidt, Sabine; Schmidt, Karin (Hrsg.) (2007): Erinnerungsorte. Deutsche Geschichte im DaF-Unterricht. Materialien und Kopiervorlagen. Berlin: Cornelsen.

Schmitt, Heiner (Hrsg.) (2010): Archive im digitalen Zeitalter. Überlieferung Erschließung - Präsentation. 79. Deutscher Archivtag in Regensburg. Fulda: Selbstverlag des Verbandes deutscher Archivarinnen und Archivare.

Schürer, Kevin: Geschichte im Internet und im ,global village“. In: Europäische Geschichtskultur im 21. Jahrhundert, hrsg. vom Haus der Geschichte der Bundesrepublik. Berlin: Nicolai, 252-261.

Schultz, Sonja (2008): Hitler 2.0. Der Diktator im Internet. In: Rother, Rainer; Herbst-Meßlinger, Karin (Hrsg.): Hitler darstellen. Zur Entwicklung und Bedeutung einer filmischen Figur. München: Edition Text und Kritik, 86-100.

Schwabe, Astrid (2010): Hypertext und Multimedia: Reflexionen zu Geschichtsdarstellungen im Internet. In: Handro, Saskia; Schönemann, Bernd (Hrsg.) (2010): Geschichte und Sprache. Berlin, Münster: Lit, 177-188.

Schwarz, Angela (Hrsg.) (2010): „Wollten Sie auch immer schon einmal pestverseuchte Kühe auf Ihre Gegner werfen?" Eine fachwissenschaftliche Annäherung an Geschichte im Computerspiel. Münster: Lit.

Spahn, Thomas (2009): Geschichte und Geschichtslernen in Zeiten des Web 2.0. Das Webportal www.lernen-aus-der-geschichte.de. In: Hardtwig; Schug (Hrsg.) (2009), 299-305.

Storch, Günther (2001): Deutsch als Fremdsprache: eine Didaktik. Theoretische Grundlagen und praktische Unterrichtsgestaltung. München: Fink. 
Storm, Monika (2010): Landesgeschichte als Gegenstand von Archivpädagogik und Historischer Bildungsarbeit. In: Dillinger, Johannes (Hrsg.) (2010): Die Vermittlung von Landesgeschichte. Beiträge zur Praxis der historischen Didaktik. Heidelberg u.a.: Verlag Regionalkultur, 101-113.

Thiemeyer, Thomas (2010): Geschichtswissenschaft: Das Museum als Quelle. In: Baur, Joachim (Hrsg.) (2010): Museumsanalyse. Methoden und Konturen eines neuen Forschungsfeldes. Bielefeld: transcript, 73-94.

Thimme, Christian (1996): Geschichte in Lehrwerken Deutsch als Fremdsprache und Französisch als Fremdsprache für Erwachsene. Ein deutsch-französischer Schulbuchvergleich. Baltmannsweiler: Schneider.

Vowinckel, Annette (2010): Mediengeschichte, Version: 1.0. In: DocupediaZeitgeschichte, 11.2.2010 (http://docupedia.de/zg/Mediengeschichte).

Warmbold, Joachim; Koeppel, E.-Annette; Simon-Pelanda, Hans (Hrsg.) (1994): Zum Thema Nationalsozialismus im DaF-Lehrwerk und-Unterricht. München: Iudicium.

Weidner, Marcus (2010): Internet und Regionalgeschichte. Die „Arbeitsgemeinschaft landesgeschichtliche und landeskundliche Internet-Portale in Deutschland“" (AG Regionalportale). In: Westfälische Forschungen 60, 617-629.

Welzer, Harald; Moller, Sabine; Tschugnall, Karoline (2002): „Opa war kein Nazi“". Nationalsozialismus und Holocaust im Familiengedächtnis. Frankfurt/Main: Fischer.

Wiedmaier, Philipp (2007): Optimierung für Suchmaschinen am Beispiel von Google: Grundlagen, Ranking, Optimierung. Saarbrücken: VDM.

Wolfrum, Edgar (1999): Geschichtspolitik in der Bundesrepublik Deutschland. Der Weg zur bundesrepublikanischen Erinnerung 1948-1990. Darmstadt: Wissenschaftliche Buchgesellschaft.

Wolfrum, Edgar (2001): Geschichte als Waffe. Vom Kaiserreich bis zur Wiedervereinigung. Göttingen: Vandenhoeck \& Ruprecht.

Wolfrum, Edgar (2006): Die geglückte Demokratie. Geschichte der Bundesrepublik Deutschland von ihren Anfängen bis zur Gegenwart. Stuttgart: Klett-Cotta.

Zierold, Martin (2006): Gesellschaftliche Erinnerung. Eine medienkulturwissenschaftliche Perspektive. Berlin, New York: de Gruyter. 



\title{
Fotografie als historische Quelle?
}

\author{
Jürgen Nielsen-Sikora
}

Geblendet schloß er die Augen.

(Elias Canetti)

\section{Einleitung}

Das Auge ist das am häufigsten zitierte Sinnesorgan, wenn es darum geht, dem Wesen des Menschen nachzuspüren. ${ }^{1}$ Schon in der griechischen Antike, die im Staunen das Motiv für Erkenntnisfortschritte entdeckte, waren existenzielle Fragen primär an die Ordnung der Blicke geknüpft. Damit einher ging seit Platon und Aristoteles die Mahnung, eine strikte Trennung von Sein und Schein (z.B. Soph. el. 1, 164a 20ff.) zu achten, um dem der Wahrheit verpflichteten Leben der theôria gerecht zu werden; ein Anspruch der Philosophie, der noch Kants transzendentale Dialektik von 1781 durchdrang und sogar bis weit in das 20. Jahrhundert hinein virulent blieb. ${ }^{2}$

Ein zentrales Kriterium der Wahrheitsfindung ist die philosophische Reflexion, jene Selbstbezüglichkeit des denkenden Ichs, das mit Descartes und Hegel zum

1 Ich danke Stephanie Coché, Martina Pilger, Uwe Koreik und Felix Hinz für inhaltsbezogene Hinweise.

${ }^{2}$ Vgl. Kant 1998. 
populären Moment des Philosophierens aufstieg und einmal mehr auf das Auge als herausragendes Organ dieser Tätigkeit verwies. Die Idolatrie und der „Adel des Sehens", auf den viele Philosophien immer wieder rekurrierten, blieben bis ins vorangegangene Säkulum uneingeschränkt an der Macht: Die Philosophischen Anthropologien von Helmuth Plessner (1928) und Ludwig Klages (1935), von Jean-Paul Sartre (1943), Hans Jonas (1979) und Hans Blumenberg (2006) kommen ohne eine Okulararistokratie nicht aus; ${ }^{3}$ unterschiedlichste Disziplinen des 20. Jahrhunderts wie die Phänomenologie, die Existenzphilosophie, die Semiotik, der Konstruktivismus oder die Ethnologie greifen Metaphern des Sehens auf und widmen sich den Fallstricken der Beobachterperspektive im Kontext der so genannten „Visual Culture“, der bildverliebten Kultur, die im „Jahrhundert der Bilder" (vgl. Paul 2008/9), der Werbung und des Konsums scheinbar allein über das Auge zur Vernunft kommt. Eine paradoxe Situation?

Bereits die Aufklärung hatte das Bild vom Menschen als Homo pictor und Animal symbolicum vorgedacht, doch erst die Spätmoderne hat diese Vorstellung vollendet: bildlich phantasierend, Bilder interpretierend, Abbilder erkennend und Zeichen verwendend wird der Mensch zu einem Wesen, das über den bildsprachlichen Ausdruck der Wahrheit auf den Grund zu gehen vermag, indem es den falschen Schein seiner Wahrnehmungswelt dekonstruiert. Doch gelingt dies nicht immer: „Ich sehe“, schrieb Roland Barthes deshalb, „den Menschen an „Bildern“ erkrankt, an seinem ,Bild" erkrankt.“ (Barthes 2006: 387). Denn das Bild, das der Mensch sich von sich selbst macht, macht er im Schatten der Bilder, die täglich auf ihn einströmen. ${ }^{4}$ In Bilderflut und Blitzlichtgewitter verliert das Selbstbild zwangsläufig an Schärfe, und der Anspruch, den der Mensch an sich seit Alters her gestellt hat, ist kaum mehr einlösbar: Der „logos“ löst sich zusehends in den Logos der Werbeindustrie auf.

Eine Kritik der Sehfähigkeit lässt ebenfalls kaum Zweifel an der Vorherrschaft des Auges: Jean Baudrillards Theorie der Simulakren (1991), die die platonische Darstellung der Troglodyten nach mehr als zweitausend Jahren konsequent zu Ende denkt, indem sie das Imaginäre als Bewusstsein gestaltendes Moment versteht, oder Vilém Flussers Entwurf einer telematischen, völlig vernetzten Gesellschaft (2007) im Informationszeitalter bilden hier keine Ausnahme.

Ein Wesen, das sich über die Jahrhunderte so intensiv und ausdauernd, ausgiebig und wohlüberlegt mit dem Blick auf die Welt, seit der Renaissance mit der Perspektivenvielfalt, seit der Weimarer Republik mit der Unschärferelation beschäftigt, muss die Erfindung der Fotografie und ihre Weiterentwicklung als digitale Computergrafik auf Grund der vermeintlichen Verdopplung der Welt als Herausforderung seiner Wahrheitssuche empfinden. Eine Neuvermessung der Blicke schien mit Heraufkunft der Fotografie unausweichlich.

\footnotetext{
${ }^{3}$ Vgl. Plessner 1978; Klages 1982; Sartre 1993; Jonas; Blumenberg 2006.

${ }^{4}$ Nota bene zeigt sich etymologisch das Verhältnis von Krankheit und Nachbildung im Begriff der „Simulation“.
} 
Inzwischen fragt auch die Geschichtswissenschaft nach dem Objektivitätscharakter fotografischer Bilder: Lassen sich Fotografien als historische Quellen nutzen, oder sind sie nicht mehr als illustratives und emblematisches Schmuckwerk in historischen Darstellungen?

Im Folgenden will ich versuchen, diese Frage anhand einiger prominenter Beispiele zu beantworten und darf vorwegnehmen, dass ich Zweifel am Nutzen des Einsatzes von Fotografien als historische Quellen hege. Allein unter der Fragestellung, wie sich der Blick auf die Welt mit der Weiterentwicklung fotografischer Techniken verändert hat, scheint mir im Hinblick auf den Stellenwert von Bildern als Quelle historischer Forschung sinnvoll. Meine Ausführungen sollen diese Skepsis argumentativ unterstreichen.

Mir geht es hierbei jedoch nicht um den didaktischen Wert der Fotografie für den Fremdsprachenunterricht, sondern allein um die Frage, ob wir durch den Gebrauch von Fotografien als Quellenmaterial in der historischen Forschung und im Geschichtsunterricht einen Mehrwert an Erkenntnis gewinnen, oder ob wir nicht gänzlich auf Fotografien im Rahmen der Geschichtsanalyse einer bestimmten Zeit, Epoche oder eines Ereignisses verzichten sollten. Meine These ist, dass sich der Mehrwert allein durch den Reiz des Anschauungsmaterials ergibt, das jeweilige Foto allerdings über die Vermittlung einer subjektiven Sichtweise in einem bestimmten Zeitraum hinaus wenig Aussagekraft besitzt. Die folgenden Bemerkungen verstehen sich insofern als Plädoyer für einen sparsamen Gebrauch der Fotografie im Kontext historischer Forschungen und erteilen der Beschäftigung mit Bildern nur dann die Legitimation, wenn es darum geht, anstelle der Aussagen über die Wirklichkeit der Vergangenheit etwas über den Blick auf diese Vergangenheit erfahren zu wollen; ein Plädoyer, Fotografien nicht bloß als Illustration des Textes, und Texte nicht nur als Kommentare der Bilder zu sehen, sondern Wort und Bild zirkulieren und sie in Dialog treten zu lassen. Allein dann, so scheint mir, haben Fotos als historische Quelle ihre Berechtigung. Hierzu will ich in drei Punkten Stellung nehmen. Zunächst sage ich etwas zum Verhältnis von Fotografie und Text (1), frage anschließend danach, was Bilder wollen (2) und gebe zum Schluss und in aller Kürze einige didaktische Anregungen, ehe ich ein Fazit meiner Ausführungen ziehe (3).

\section{Zum Verhältnis von Fotografie und Text}

Stellen Sie sich angesichts des oben skizzierten Vorhabens einmal eine Situation vor, in der jemand ein Foto wie folgt beschreibt:

„Das Bild ist schwarz-weiß. Ich sehe ein Schlachtfeld, und auf dem Schlachtfeld einen einzelnen Mann, der zwischen gefallenen Soldaten steht und entsetzt in die Weite blickt. Er fasst sich mit der rechten Hand an den Kopf und reißt Augen und Mund weit auf. Die andere Hand liegt auf der Schulter eines am Hang liegenden gefallenen Soldaten. Er hat einen langen Mantel an, der nicht 
geschlossen ist und trägt einen Gürtel und Stiefel. Außerdem hat er eine Box um den Hals gehängt. Die ganze Szene scheint in einem Schützengraben zu spielen. Die gefallenen Soldaten liegen zum Teil übereinander, zwei haben Blut im Gesicht. Alle Soldaten bis auf den Überlebenden tragen Helme und haben die Augen geschlossen. Insgesamt ist aber nicht erkennbar, ob auch alle liegenden Soldaten wirklich tot sind. Die Gesichter der Männer sehen relativ jung aus, in jedem Fall deutlich jünger als das des entsetzt in die Weite blickenden Mannes. Zwischen den Soldaten, vor dem lebenden Mann, liegen nicht weiter erkennbare Gegenstände. Außer dem Schützengraben ist nichts zu sehen. Keine Natur, keine Gebäude, keine anderen Menschen, keine schweren Geschütze.“

Mit diesen Worten skizzierte eine Kollegin ein Bild, das ich ihr mit der Bitte überreichte, sie möge die Szene in Worte fassen. Wann und wo das Gesehene spielt, lässt sich scheinbar nicht exakt sagen. Meine Kollegin äußerte die Vermutung, es sei ein Bild aus dem Ersten Weltkrieg. Der Stahlhelm, der Schützengraben ließen darauf schließen.

Warum die Bildbeschreibung? „Bilder sind längst fester Bestandteil in verschiedenen Bereichen des Lehrens und Lernens" schreiben Hecke und Surkamp (2010: 9). Hierbei gehe es um die Fragen nach Bildauswahl, Verwendung und methodischem Einsatz. Die Bilder und Fotografien einerseits, die Reflexion des Einsatzes andererseits bleiben im Unterricht jedoch oftmals unkritisch. Auch Hecke und Surkamp neigen meines Erachtens zu einer Überbewertung der Möglichkeiten des Bildeinsatzes. So zeichne sich eine Abbildung dadurch aus, „dass die zentralen Merkmale von Darstellung und Dargestelltem übereinstimmen (wie z.B. bei Porträtfotos)“ (ebd.: 10). Des Weiteren könnten ,authentische Bilder ... produktive Sprechanlässe liefern“ (ebd.: 11) und seien „wertvolles Material“ (ebd.) für den Unterricht, weil sie diesen positiv beeinflussen und „das Textverständnis unterstützen" (ebd.: 12) würden. Insgesamt ließe sich das Sehen trainieren und trüge zu einer "Medienkompetenz der Lernenden“, zur „Sehkompetenz" und „visual literacy“ bei (ebd.: 13). Trainiert werden solle insbesondere die „Fähigkeit, Bildinhalte verbalisieren zu können“ (ebd.: 14). Ein visuell kompetentes Betrachten unterscheide sich vom bloßen Wahrnehmen dadurch, „dass die visuellen Reize kritisch begutachtet und bewusst unter Bezugnahme auf Gestaltungshinweise und Kontext des Bildes gedeutet werden.“ (ebd.: 15). Der letzte Punkt, auf den auch Gabriele Blell aufmerksam macht, ist entscheidend. Zwar gehe es darum, so Blell, den Lerner zu einem kenntnisreichen und kritisch-kompetenten „Leser im Sinne eines Schauers aller Arten von visuellen Texten“ (ebd.: 94) zu machen, weil alles Visuelle eine bedeutungsstiftende, eine bedeutungstragende sowie eine bedeutungsvermittelnde Instanz“" (ebd.: 95) besitze. Gleichwohl gibt sie zu verstehen, dass Lesen und Sehen „immer subjektiv, selektiv, also immer schon Interpretation“ (ebd.: 98) der jeweiligen „Bildgrammatik“ seien. Die Schwierigkeit, diese Bildgrammatik kritisch zu entschlüsseln, sollte nicht unterschätzt werden.

Kommen wir deshalb noch einmal auf die Bildgrammatik der eingangs beschriebenen Szene zurück. Die in Worte gefassten Eindrücke meiner Kollegin 
bilden den Ausgangspunkt für ein kleines Gedankenexperiment des Bildverstehens: Zu Beginn eines Vortrags lese ich den Text vor, ohne das Bild, das die Kollegin beschreibt, zu zeigen. Anschließend bitte ich meine Zuhörer, sich vor dem inneren Auge die Situation vorzustellen. Erst danach wird die Fotografie gezeigt. Die Diskrepanz zwischen all den Vorstellungen, die sich durch die Beschreibung ergeben und dem auf die Leinwand projizierten Bild ist, wie zu erwarten, groß. Denn der Versuch, das Foto in Worte zu fassen, kann noch so gelungen sein, eine Übereinstimmung zwischen der Gedankenwelt und der Sehwelt ist nicht herzustellen; auch dann nicht, wenn weitere Personen die Bildbeschreibung durch eigene Worte ergänzen und komplettieren würden. Aussagen zum Format, zum Mittelpunkt des Bildes, zum Vorder- und Hintergrund oder zu den Bildachsen mögen hierbei Konkretisierungen des inneren Bildes hervorrufen, doch lassen sich niemals alle Bildinformationen als Text rekapitulieren. Kurt Tucholsky fasste diesen Sachverhalt bereits im Jahre 1926 wie folgt zusammen: „Weil jeder genau so ist, wie er aussieht, und weil wir nur nicht lesen können, was uns die Natur eindeutig auf die Menschengesichter schreibt, so können Augenblicksfotografien erbarmungslos enthüllen, was das Auge nicht so schnell hat wahrnehmen können. Eine Momentaufnahme ist die fixierte Blamage einer unvorsichtigen Bewegung, eines schiefen Lächelns, einer sorgsam versteckten Beobachtung ... Plötzlich ist alles am Tage ... Und weil ein Bild mehr sagt als hunderttausend Worte, so weiß jeder Propagandist die Wirkung des Tendenzbildes zu schätzen: von der Reklame bis zum politischen Plakat schlägt das Bild zu, boxt, pfeift, schießt in die Herzen und sagt, wenn's gut ausgewählt ist, eine neue Wahrheit, und immer nur eine. Es gibt Beschreibungen, die die Bilder übertreffen, aber das ist selten. Es gibt hunderttausend Fotografien, die den besten Schilderer übertreffen, das ist die Regel ...“ (Peter Panter 1926: 75).

Das von mir ausgewählte Bild war ein Fotostill, ein spiegelverkehrt aus dem Internet kopiertes Cover aus G.W. Pabsts UFA-Klassiker „Westfront 1918“ aus dem Jahre 1930, aus dem ich alle Textbausteine getilgt hatte, so dass kein Verweis auf den Film mehr sichtbar war. Erst im Vergleich mit dem vollständigen Cover wird deutlich, dass das Foto nicht aus dem Ersten Weltkrieg stammt, sondern am Ende der Weimarer Republik produziert wurde. Das Foto war gestellt und verfälscht. Diese Informationen erhalten wir aber nur durch den Text, der auf die Klassiker-Edition, die Schauspieler und den Regisseur verweisen. Das Bild, das ich eingangs der Kollegin gezeigt habe, sagt mithin mehr als tausend Worte; es sagt aber zugleich auch weniger. Zum Verständnis der dargestellten Szene benötigen wir stets Informationen, die sich nicht allein aus dem Anblick eines Fotos erschließen. 


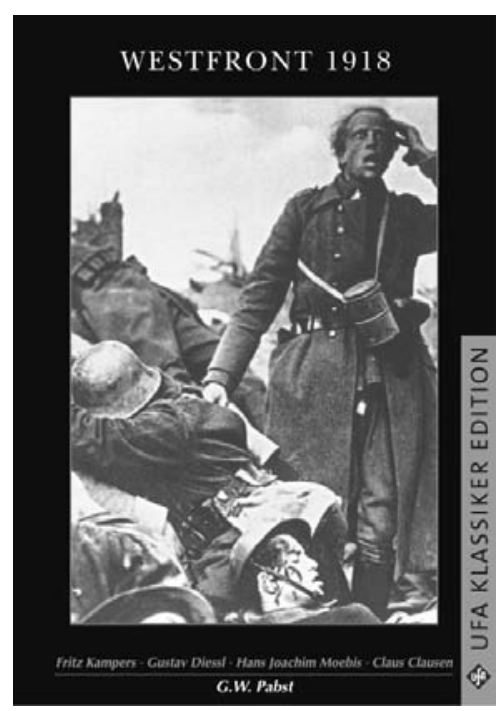

Abb. 1: DVD-Cover von G.W. Pabsts „Westfront 1918“, (C) Edition Ufa-Klassiker.

Auch der Pionier der Fotografie, William Henry Fox Talbot, der in seinen Bildern durchaus einen historischen Mehrwert erblickte und die Fotografie 1844 als „Zeichenstift der Natur“ (vgl. Stiegler 2010: 161) begriff, kam nicht ohne Kommentare zu seinen Bildern aus. Seine Schilderungen fielen dabei recht bildreich aus. So heißt es über das Queen's College in Oxford: „Die Oberfläche des Gebäudes mit ihrem verwitterten Stein, der wahrscheinlich von Anfang an von schlechter Qualität war, zeigt aufs deutlichste Spuren der Zerstörung durch Zeit und Wetter.“ (161). Über den Pariser Boulevard heißt es: „Es ist Nachmittag. Die Sonne verlässt gerade die säulengeschmückte Häuserreihe: Die Fassade ist schon im Schatten, aber ein einzelner Fensterladen steht weit genug offen, um einen Schimmer Sonne zu erhaschen ... Ein wahrer Wald von Schornsteinen säumt den Horizont: Denn das Instrument registriert alles, was es wahrnimmt, und einen Schornsteinaufsatz würde er mit der gleichen Unparteilichkeit festhalten wie den Apoll von Belvedere.“ (162f.). Das Instrument ist der Fotoapparat, dessen „Unparteilichkeit“ Talbot heraufbeschwor. Doch ist dies eine Illusion: Die Natur kennt keine Rahmung, keinen Ausschnitt und keine Welt in Schwarz und Weiß. Die Perspektive eines Fotos ist zudem eine andere als die des Blicks; auch verteilt sich die Sehschärfe anders als auf der Fotografie: Wir sehen nur im Blickzentrum scharf, an den Rändern unseres Blicks wird die Welt zusehends unschärfer. Aber nicht nur hier täuscht uns das fotografische Bild - es verschweigt auch den Kontext, in den es eingebettet ist: Seinen möglichen Auftraggeber, die Entstehungszeit, den Ereigniszusammenhang, die Agentur, die das Foto vertreibt, die Auflagenhöhe und die ganze Bilderpolitik, die im Zuge seiner Verwendung und Nichtverwendung betrieben wird (vgl. Jäger 2009). Viele Fragen bleiben offen bei einer 
Bildbetrachtung: Wer sind die abgebildeten Personen? Wie wurde das Bild rezipiert? Welche Bedeutung hatte es für seine Betrachter? Wie Susan Sontag schreibt (vgl. Stiegler 2010: 295), kann ein Foto kein Beweis sein für das, was passiert, ohne dass wir über ein politisches Bewusstsein der Kontexte des Geschehens verfügen: Welche Intention hatte der Fotograf? Wie wirkt das Bild auf seine Betrachter? Welche Assoziationen verbinden sie damit? Und in welchem Verhältnis steht das Bild zu den Ereignissen, von denen es einen Ausschnitt widerspiegelt?

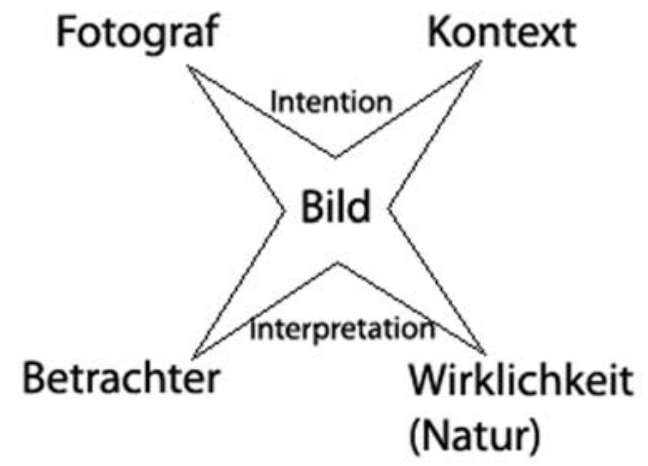

\section{Abb. 2: Bild-Kontexte}

Jedes Foto wird aus einem Kontext gerissen, den es zu rekonstruieren gilt, will man Genaueres über das auf dem Foto Dargestellte wissen. Der Ausschnitt, den das Foto zeigt, mag zufällig und spontan gewählt worden sein; selbst dann wird man dem Fotografen noch Absicht und insofern eine Intention unterstellen dürfen, die es zu interpretieren gilt wie einen Text. Dieser semiotisch anders zu deutende visuelle Text, der uns in jedem Foto vorliegt, steht im Spannungsfeld von Bildintention und Bildinterpretation des jeweiligen Betrachters. Aussagen über den Bildkontext lassen sich stets leichter verifizieren als Spekulationen über das Verhältnis zwischen Abbild und Wirklichkeit: Ist der Mensch auf dem Foto unten Barack Obama oder doch George W. Bush? 


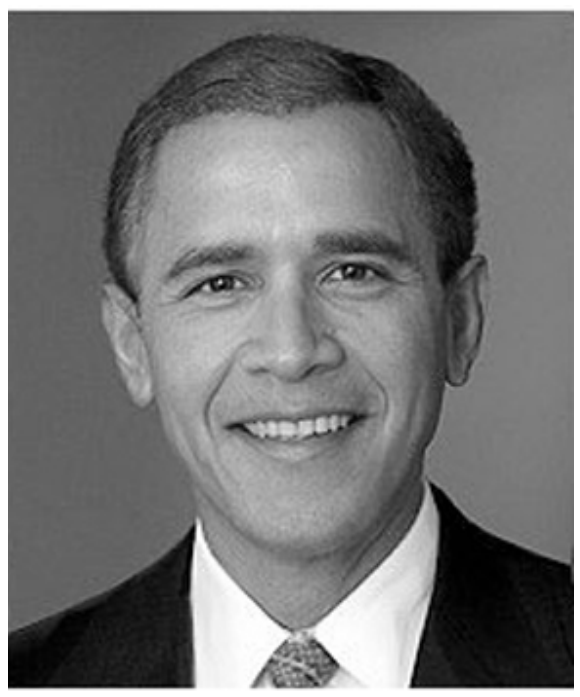

\section{Abb. 3: Gemorphtes Bild aus den Konterfeis von Barack Obama und George W. Bush}

Fotografien sind mithin nicht nur bedeutungstragend, wie Blell schreibt, sondern vor allem bewusstseins- und meinungsbildend, weil jedes Foto im Zeitalter der digitalen Computergrafik prinzipiell manipuliert werden kann. Die Manipulation muss nicht einmal gravierend sein. Ein prominentes Beispiel ist das 1972 geschossene Foto der damals neunjährigen Vietnamesin Kim Phuc aus dem zweiten Indochina-Krieg. Es zählt wohl zu den bekanntesten Fotografien des 20. Jahrhunderts und hat nicht zuletzt medienpolitisch auf die damaligen Gesellschaften in den USA und Europa eingewirkt. Das Foto wurde nachträglich beschnitten; so rückt die völlig verbrannte, nackte, weinende Kim Phuc stärker in den Bildmittelpunkt, der zwischen ihrem Nabel und der Scham liegt. Sie ist so zu einer Bildikone des vergangenen Jahrhunderts geworden.

Aber genau dies ist auch ein zentrales Problem historischer Aufnahmen: Die Geschichte zerfällt, so schon Walter Benjamin in seinem Kunstwerkaufsatz von 1935, in Bilder. Und Susan Sontag ergänzt: „Bilder lähmen. Bilder betäuben ... Je öfter man mit solchen Bildern konfrontiert wird, desto weniger real erscheint das betreffende Ereignis." (Benjamin 2010: 296f.). Denn der politische und gesellschaftliche Diskurs verlagert sich durch die Fotografie, besser: durch die permanente Reproduktion von Fotografien auf die Ebene einer zweiten Realität. Diese Ebene vermittelt nichts als subjektive Sichtweisen und verfolgt bestimmte Ziele. Die Frage, die wir uns deshalb stellen müssen, wenn wir Bilder als historisches Dokument betrachten, lautet: Was wollen Bilder? 


\section{Was wollen Bilder? Einige Beispiele}

Bernd Stiegler weist in diesem Zusammenhang darauf hin, dass Fotografien in erster Linie nicht die Wirklichkeit verdoppeln - und insofern als historische Quelle unbrauchbar sind, um etwas über die Inhalte des Dargestellten zu erfahren, vielmehr konstituiere die Fotografie „das Bild der Wirklichkeit. Es geht weder um Naturalismus noch um Konstruktivismus, sondern um eine in der Fotografietheorie und mitunter sogar in den Bildern medial vermittelte Reflexion über Konstruktivismus und Naturalismus ... darüber, was zu bestimmten Zeiten und in bestimmten Kontexten als Wirklichkeit und als visuelle Wahrheit zu fassen ist. Dies ist notwendig historisch zu bestimmen: als Geschichte der Wahrnehmung, des Realismus und der Konstruktionsformen des Realen." (Stiegler 2010: 23f.). Es geht mithin darum, wie eine bestimmte Zeit Wirklichkeit konstruiert hat, und welchen Beitrag zu dieser Konstruktion des Realen die Fotografie auf einem bestimmten Stand der Technik lieferte. Zwar ist das Foto des kleinen Mädchens zum Inbegriff des Vietnamkrieges und seiner Gräuel geworden, und es steht wie kein anderes Dokument für die verheerende Wirkung der Napalmbomben. Über die Hintergründe und die Geschichte des Krieges aber sagt es nichts; schon gar nichts darüber, warum die Vietnamesen, die die US-Bombe geworfen haben, ihr eigenes Volk angriffen.

An dieser Stelle darf man gewiss einwenden, dass auch ein so großartiges Buch wie Bernd Greiners „Krieg ohne Fronten“ (2009), das bis dato unbekanntes Archivmaterial zum Vietnamkrieg präsentierte, nicht lückenlos sein kann. Doch das Wort ist in der Lage zu reflektieren, wohingegen das Foto immer nur ein „Bruchstück der Welt“ sein kann; eine Miniatur der Realität, „die jedermann anfertigen oder erwerben kann." (Sontag a.a.O.: 278). Das Foto wird auf Grund seines Reproduktionscharakters zu einer Projektionsfläche, für die allein die Aufmerksamkeit des Betrachters entscheidend ist. 


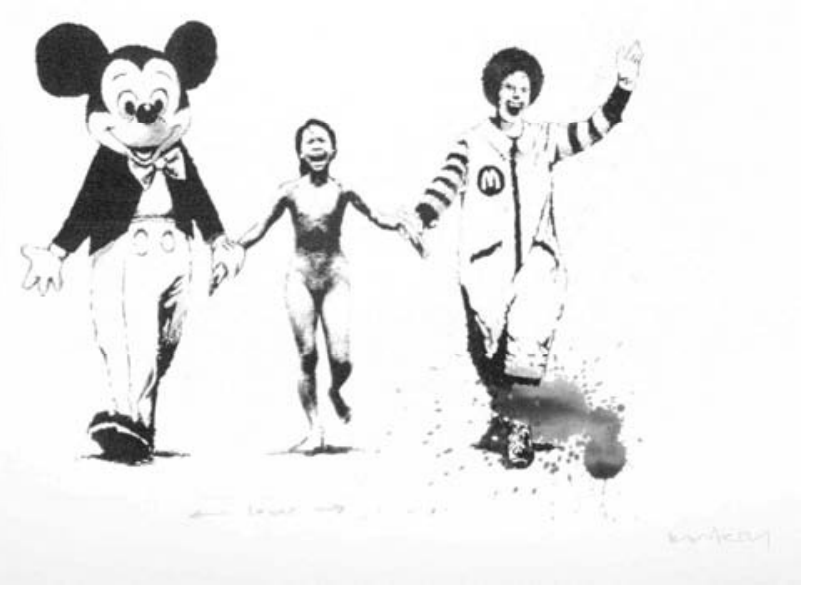

Abb. 4: Kim Phuc als Pop-Ikone zwischen Mickey Mouse und Ronald McDonald. Ein Werk des Graffiti-Künstlers Banksy.

Immer und immer wieder gezeigte Bilder sind Indizes von Wendepunkten und Umbrüchen (vgl. Schuster 1996: 194). Die Erinnerung an fotografische Repräsentationen ist vitaler als das Erlebte. „Politics of Representation“ könnte man im Anschluss an Victor Burgin (1982) diese Form der medialen Inszenierung nennen und zugleich unterstreichen, dass Fotos alles andere als ein „Fenster zur Welt" (vgl. Burgin in Wolf 2002: 25) darstellen. Vielmehr, so Burgin, gehe es darum zu zeigen, ,inwiefern die Repräsentationsmittel das Repräsentierte determinieren.“ (26). Eine dekonstruktive Lektüre des immer stärker in den Vordergrund rückenden Hilfsmittels der Geschichtswissenschaft ist es, worum sich Burgin bemüht: „Die Oberfläche des Fotos aber verbirgt nichts außer der Tatsache der eigenen Oberflächlichkeit." (37). Wo sie doch einmal durchbrochen wird, dort spielt sie selbstironisch auf ihren Oberflächencharakter an. So zum Beispiel in Michael Schirners Fotomontage von Brandts Kniefall 1970 in Warschau. Das digital bearbeitete Foto kommt ganz ohne den Protagonisten aus: Brandt fehlt auf Schirners Foto; der leere Fleck lädt das Foto noch einmal mit Bedeutung auf, indem es den Ikonencharakter der Darstellung und des Nicht-Dargestellten kritisch befragt. Jean Baudrillard sprach in den Fatalen Strategien (1991) von der „Kunst des Verschwindens“. Wenn diese irgendwo geglückt sein sollte, dann am ehesten in diesem großartigen Foto von Michael Schirner. 


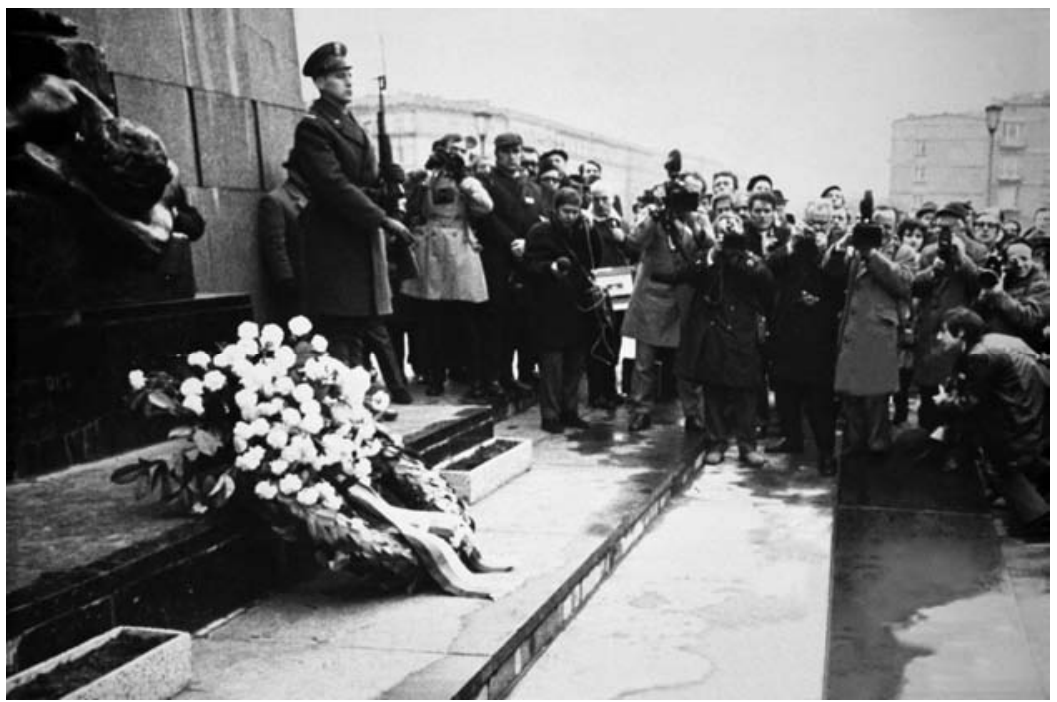

Abb. 5: (c) Michael Schirner, Bye bye.

Quelle: http://www.michael-schirner-bye-bye.de/site/page/byebye

„Wir entfernen 100\% von allem, was das ursprüngliche Photo bekannt gemacht hat: den Protagonisten, das zentrale Bildmotiv, Form und Inhalt der Geschichte. Das durch Entfernen des Bildvordergrundes entstandene »Loch« oder die Leerstelle wird in aufwendigen Verfahren durch digitales Neumalen des Hintergrundes gefüllt. So entstehen ein neues Bild und ein selbständiges Werk. Ziel der Unsichtbarmachung oder des Entfernens vom ursprünglichen Inhalt und der wesentlichen Elemente des Bildes ist es, aus dem journalistischen Photo etwas kategorisch Anderes zu machen..." (ebd.).

Schirner hat Roland Barthes' Idee der Punktierung gewissermaßen ins Negativ gekehrt. Barthes sprach 1980 in „Die helle Kammer“ von den empfindlichen Stellen und Verletzungen eines Fotos. Diese nennt er ,punctum“ und deutet sie als „Strich, kleines Loch, kleiner Fleck, kleiner Schnitt - und: Wurf der Würfel. Das punctum einer Photographie, das ist jenes Zufällige an ihr, das mich besticht (mich aber auch verwundert, trifft)." (Barthes 1989: 36). In Schirners digital bearbeiteten Fotografien wird die Verwunderung dadurch hervorgebracht, dass der uns eigentlich bekannte Stich (die Verwundung) ausgelöscht wird. Somit wird das Punctum gleichwohl besonders betont - als Negativ. Wenn für Barthes das Punctum etwas ist, das ihn der Fotografie etwas hinzufügen lässt, so fügt Schirner dem Bild etwas hinzu, indem er etwas weglässt.

Schirners Bildkritik - vermittelt durch das Bild selbst - ist ungewöhnlich und neu. Textkritik am vermeintlichen Repräsentationscharakter der Fotografie kam hingegen bereits im 19. Jahrhundert auf. Auch waren Bearbeitungen der Fotos durchaus üblich. Nicht alle teilten Talbots Auffassung vom Zeichenstift der Natur. 
Neben Johann Gustav Droysens Historik aus dem Jahre 1857, die kritisch auf die Fotografie als historische Quelle blickt ${ }^{5}$, war es beispielsweise Peter Henry Emerson, der Arzt und Fotograf, der Ende des 19. Jahrhunderts bemerkte, Fotos spiegelten keinesfalls die Wahrheit einer Szene wider. Das menschliche Auge, so Emerson, sehe nur eine Anzahl von Zeichen. Nur aus Gewohnheit würden wir diese Zeichen „Natur“ nennen. Ein wahrer Eindruck einer Szene sei nicht erkennbar (vgl. Emerson in Stiegler: 168f.).

Unsere Sehgewohnheiten widersprechen dieser Einschätzung allerdings bis heute, denn das Foto ist ,begrifflich nicht von seinem Trägermaterial zu trennen. Phänomenologisch schlägt sich das Foto als reines Bild nieder, und dieser Effekt ist auch der Grund dafür, weshalb wir der Fotografie gewöhnlich den mythischen Wert der Transparenz zuschreiben“, merkt Solomon-Godeau an (in Wolf 2003: 70) und formuliert als Aufgabe, diese ,encodierten und verborgenen Bedeutungen ans Licht zu bringen, die historischen und formalen Strategien aufzudecken, die die Herstellung, den Sinn, die Rezeption und den Gebrauch einer Arbeit determiniert haben ..." (72). Angesichts der Bilderflut heutzutage wirkt diese Herausforderung allerdings alles andere als einfach und kann nur im Einzelfall zum Erfolg führen, um paradigmatisch den grundsätzlich dubitativen Charakter (Lunenfeld) von Fotografien zu demonstrieren. Doch Zweifel am Wahrheitsgehalt von Fotografien sind bis heute nicht üblich. Wir müssen hierbei nicht einmal Fotos zitieren, die der damalige Außenminister der Vereinigten Staaten der Weltöffentlichkeit präsentierte, um den Irak-Krieg zu rechtfertigen. Fotos, auf denen zwar nichts wirklich zu sehen war, die aber beweisen sollten, dass der Irak über Massenvernichtungswaffen verfüge. Wir brauchen nur einmal in einige Schulbücher zu schauen und stellen fest, wie selbst in Unterrichtsmaterialien der historische Kontext manipuliert wird. In einer gängigen Illustration einer spartanischen Phalanx wurde der fünfte Krieger wegretuschiert, weil er in sich zusammensackt und scheinbar nicht dem Wesen der Phalanx entspricht. Seine Beine aber blieben erhalten, weil sie im Gewühl der übrigen Krieger auch nicht weiter auffallen. Dass die vier Spartaner in der Bildmitte über zu viele Beine verfügen, stört viele Schulbuchverlage nicht.

${ }^{5}$ Vgl. Droysen 1977. 


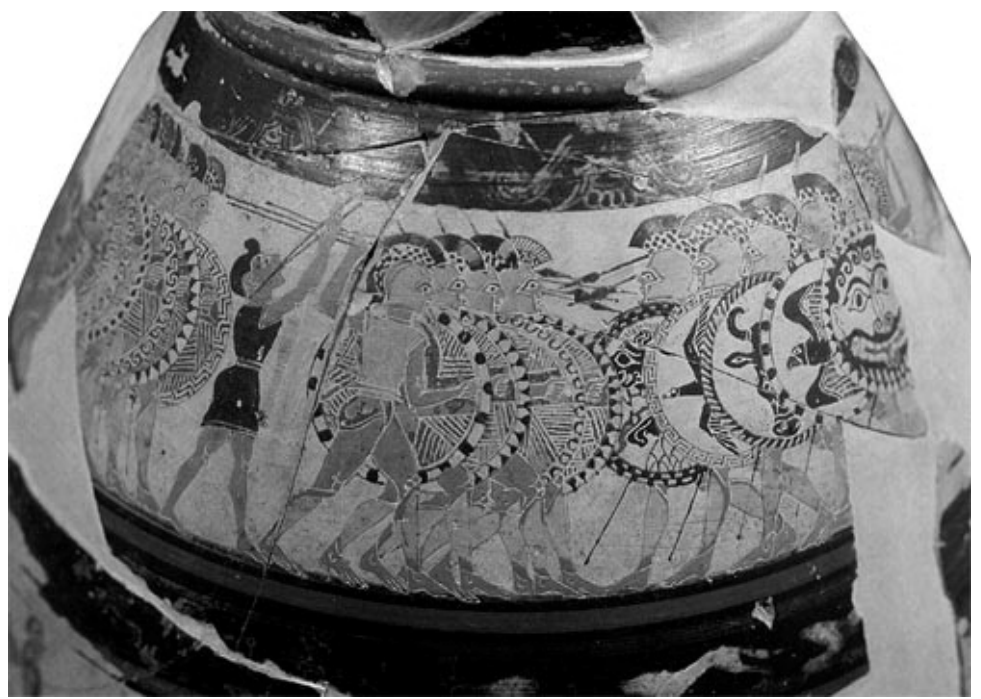

Abb. 6: Spartanische Phalanx, der gefallene Krieger wurde wegretuschiert. Nur seine Beine sind noch am Platz. Forum Geschichte (Cornelsen), Bd. 1, 2005: 89.

Es sind solche durch Manipulation hervorgerufene Bedeutungsverschiebungen, die im Rahmen einer Quellenanalyse in erster Linie in den Blick genommen werden müssen. In den Blick nehmen bedeutet, dass das betrogene Auge fähig ist, den Trug zu entlarven. Eine reflexiv-kritische Lektüre visueller Texte kann den über die Fotografie vermittelten Schein der Dinge wieder ins rechte Licht rücken. Den falschen Schein erkennen, der sich nicht nur in gemorphten, gefilterten, weich gezeichneten, gespiegelten, invertierten, gedrehten, gestreckten, kolorierten oder gerasterten Bildern, sondern in ausnahmslos jedem Foto wieder findet, kann nur, wer die Augen ein weiteres Mal bemüht, genauer hinzusehen und Textinformationen hinzuzieht. Denn die Kamera setzt zunächst alles gleich und ebnet die Bedeutungen ein. Sie hat, wie Susan Sontag zu Recht konstatiert, eine „chronisch voyeuristische Beziehung zur Welt geschaffen." (Sontag, a.a.O.: 286).

Andererseits kann sie Dinge sichtbar machen, die für das bloße Auge unsichtbar sind. Denken wir nur an eine Torkamera, an das Zielfoto bei einem 100 Meter-Sprint und an andere Bewegungsabläufe, die mittels Fotografie exakter zu studieren sind, weil sie die Welt für den Bruchteil einer Sekunde einzufrieren vermögen und, wie Jean Baudrillard skizziert, „das Überstürzen der Ereignisse“ unterbrechen (a.a.O., 56). Doch diese Ereignisse müssen zuvor definiert worden sein: Wembley, Olympische Spiele, 11. September etc. Erst nach ihrer Definition und der Festlegung ihres Status im Kontext der Bilderpolitik können Fotos eine Kontrolle über unsere Erinnerungen ausüben: Nine-Eleven wird sodann zum Synonym für die ewige Wiederkunft des Bildes von Flug 175 der United Airlines. 
Wie Georges Salles meint, ist die Erinnerung ein „geheimnisvolles Auge“, das „wiederkäut“. (Salles 2001: 48).

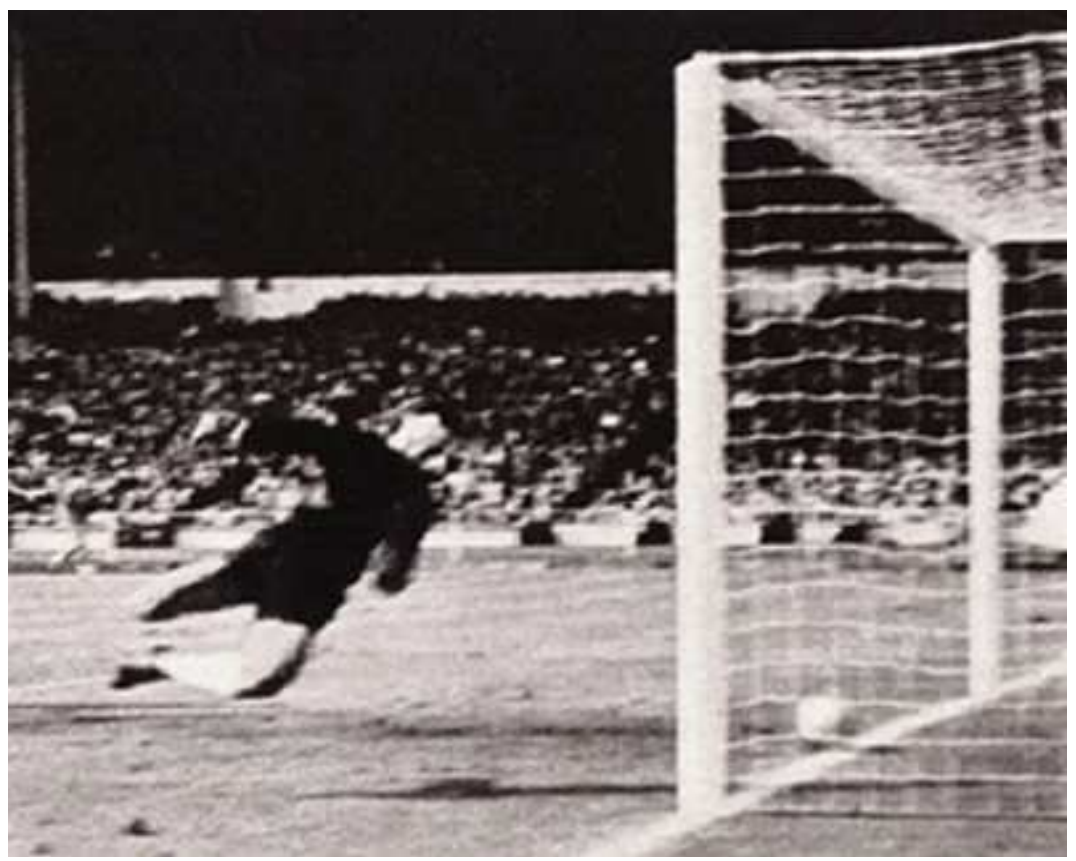

Abb. 7: Wembley-Tor 1966: Fotografie als (Gegen-) Beweis?

\section{Didaktische Aspekte und Fazit}

Der Mensch ist dieses Wesen, das seine inneren Bilder stets wiederkäut und versucht ist, über dieses Wiederkäuen eine Kontrolle über die Vergangenheit zu erlangen. Sich der Geschichte zu vergewissern über die Bilder, die wir von ihr besitzen, sie in der Gegenwart wieder aufblitzen zu lassen, daran scheint kein Weg vorbei zu führen, sucht der Mensch in seiner Gegenwart Orientierung. Nicht zuletzt gilt dies für Erinnerungen an sein eigenes, gelebtes Leben: „Ach, das war ich, als ich so und so alt war, schau. Und hier, auf diesem Bild, bin ich mit Vater und Mutter in Frankreich. Das hier ist der Hof meines Großvaters und auf dem nächsten Bild ..." Ohne die Fotografien gäbe es diese Erinnerung nicht; nicht in der Form. Die Bilder prägen unsere Erinnerung an die Vergangenheit, die wir durch ihre Brille lesen: Habe ich das wirklich so erlebt, oder erlebe ich das jetzt nur so, weil ich mir dieses Foto nach Jahren wieder anschaue? Kann ich mich nur erinnern, weil ich dieses Bild von der Vergangenheit in Händen halte? „Die Fotografie befreit ... die bildhaften Erinnerungen aus ihrer absoluten Isolation, indem 
sie über ein Foto kommunizierbar werden" schreibt der Kölner Psychologe Martin Schuster (a.a.O.: 17). In der Fotografie sieht er ein technisches Instrument, das die Möglichkeiten unserer Denkgewohnheiten selbst verändere und zur Bewusstseinserweiterung beisteuere. Noch stärker als die Erinnerung an bestimmte Personen sei allerdings die Erinnerung, die fotografierte Dinge übermittelten (ebd.: 76): Der Teddy, mit dem man früher gekuschelt, das Auto, das man einst gefahren, oder das Zimmer, das man vor langer Zeit bewohnt habe. Dem pflichtet auch Pierre Bourdieu bei, indem er fragt: „Was ist realer und der Realität getreuer als eine Photographie? Was ist beruhigender und deutlicher? Und zwar derart, dass die Photographien einen zur Verzweiflung bringen: Wenn es regnet, wozu sollte man sagen, dass es regnet?“ (Pierre Bourdieu, zitiert nach: Brohm 2009: 197).

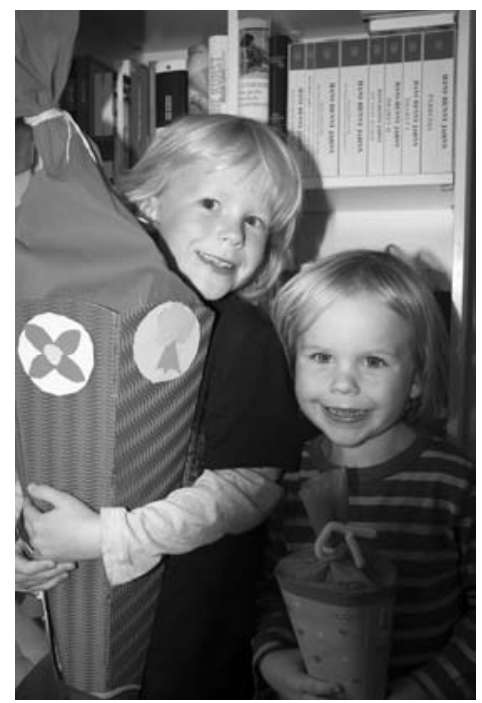

Abb. 8: Waren wir das damals auf diesem Bild? Erinnern wir uns an die Situation auf Grund des Fotos? Konservieren wir darin unsere Erinnerung? (Der 1. Schultag, (C) Verfasser 2010)

Ziehen wir ein Fazit unserer Überlegungen. Wir haben feststellen müssen, dass eine Fotografie unkommentiert niemals ein Zitat der Wirklichkeit sein kann. Sie ist insofern als Quelle, die etwas über die Wirklichkeit der Geschichte aussagt, zunächst ungeeignet. Die Fotografie, sagt Jean Baudrillard, sei „unser Exorzismus. Die primitive Gesellschaft hatte ihre Masken, die bürgerliche Gesellschaft ihre Spiegel. Wir haben unsere Bilder ... Durch das Bild erzwingt die Welt ihre Diskontinuität, ihre Zerstückelung, ihre künstliche Augenblicklichkeit.“ (Baudrillard in Stiegler, a.a.O.: 50f.). Allerdings scheint die Fotografie als Quelle, die etwas über die Wirklichkeit der Blickpunkte in der Vergangenheit und Gegenwart aussagt, geeignet, ihren Beitrag im Rahmen historischer Forschung zu leisten. Denn sie 
vermittelt subjektive Sichtweisen und ihre Veränderungen (vgl. Warnke et al. 2011). Darin liegt jedoch auch die einzige Legitimation der Verwendung von Fotografien als historische Quelle. Nicht das dargestellte Geschehen als solches, sondern allenthalben die Ordnung der Blicke (vgl. Reich 1998) auf das Ereignis ist es wert, untersucht zu werden. Darüber hinaus beeinflussen Fotografien unsere Erinnerungen. Manche Ereignisse werden nur deshalb in Erinnerung behalten, weil sie durch das Betrachten eines Fotos mehrmals als Erinnerungsbild reproduziert werden: Das Foto nimmt den Platz der realen Situation ein. Hierbei eröffnet das, was Roland Barthes (1980/89) das Punctum der Fotografie genannt hat, die Stelle im Bild, die uns zur Verwunderung, zum Staunen bringt, neue Zugänge zur (eigenen) Geschichte. Denn Fotos verändern Sehgewohnheiten. Diese Veränderungen im „kollektiven Bildbewusstsein“ (Schuster) müssen thematisiert werden. Nehmen wir das seit den Griechen so oft beschworene Staunen über die Bilder der Welt einmal mehr zum Anlass, um auch und vor allem im Geschichtsunterricht die Frage nach dem Menschen und den Bildern, die er sich von sich selbst macht, nachzugehen.

\section{Literatur}

Aristoteles (1883): Sophistische Widerlegungen. Übersetzt von J. H. von Kirchmann, Heidelberg: Georg Weiss.

Barthes, Roland (2006): Das Rauschen der Sprache. Kritische Essays. Frankfurt/Main: Suhrkamp. (Original 1977).

Barthes, Roland (1989): Die helle Kammer. Bemerkungen zur Photographie.

Frankfurt/Main: Suhrkamp. (Original 1980).

Baudrillard, Jean (1991): Die fatalen Strategien. Berlin: Matthes \& Seitz.

Benjamin, Walter (2010): Das Kunstwerk im Zeitalter seiner technischen Reprodurierbarkeit. Frankfurt/Main: Suhrkamp. (Original 1935).

Blell, Gabriele (2010): Der Leser als „Grenzgänger“: Entwicklung intermedialer Lese- und Sehkompetenzen. In: Hecke; Surkamp (2010), S. 94-109.

Blumenberg, Hans (2006): Beschreibung des Menschen. Frankfurt/Main: Suhrkamp.

Bourdieu, Pierre (2009): L'image de l'image. Zitiert nach: Holger Brohm: Diagramm und Fotografie als Praxis des Visuellen. Pierre Bourdieu (1930-2002). In: Jörg Probst; Jost Philipp Klenner (Hrsg.): Ideengeschichte der Bildwissenschaft. Siebzebn Porträts. Frankfurt/Main: Suhrkamp, S. 197-213.

Burgin, Victor (1982): Thinking Photography. London: Macmillan Education.

Droysen (1977): Historik. Historisch-kritische Ausgabe. Hg. von Peter Leyh. Stuttgart-

Bad Cannstadt: Fromann-Holzboog. (Original 1857). 
Flusser, Vilém (2007): Kommunikologie. Frankfurt/Main: Fischer.

Greiner, Bernd (2009): Krieg ohne Fronten. Die USA in Vietnam. Hamburg: Hamburger Edition.

Hecke, Carola; Surkamp, Carola (Hrsg.) (2010): Einleitung: Zur Theorie und Geschichte des Bildeinsatzes im Fremdsprachenunterricht. In: Dies.: Bilder im Fremdsprachenunterricht. Neue Ansätze, Kompetenzen und Methoden. Tübingen: Narr, S. 9-24.

Jäger, Jens (2009): Fotografie und Geschichte. Frankfurt/Main: Campus.

Kant, Immanuel (1998): Kritik der reinen Vernunft. Hrsg. von Jens Timmermann. Hamburg: Meiner. (Original 1781).

Klages, Ludwig (1982): Grundlegung der Wissenschaft vom Ausdruck. Bonn: Bouvier. (Original 1935).

Paul, Gerhard (Hrsg.) (2008/9): Das Jabrhundert der Bilder. 2 Bände. Göttingen: Vandenhoeck \& Ruprecht.

Platon (1988): Der Staat, übersetzt von Otto Apelt. Hamburg: Meiner.

Plessner, Helmuth (1978): Die Stufen des Organischen und der Mensch. Einleitung in die philosophische Anthropologie. Berlin: de Gruyter. (Original 1928).

Reich, Kersten (1998): Die Ordnung der Blicke. 2 Bände. Neuwied: Luchterhand.

Salles, Georges (2001): Der Blick. Berlin: Vorwerk 8. (Original 1939).

Sartre, Jean-Paul (1993): Das Sein und das Nichts. Versuch einer phänomenologischen Ontologie. Reinbek b. Hamburg: Rowohlt. (Original 1943).

Schuster, Martin (1996): Fotopsychologie. Lächeln für die Ewigkeit. Berlin und Heidelberg: Springer.

Solomon-Godeau, Abigail (2003): Wer spricht so? Einige Fragen zur Dokumentarfotografie. In: Wolf (Hrsg.) (2002): 53-74.

Stiegler, Bernd (2010): Texte zur Theorie der Fotografie. Reclam: Ditzingen.

Tucholsky, Kurt alias Peter Panter (1926): Ein Bild sagt mehr als tausend Worte. In: Uhu 2, November 1926: 75.

Warnke, Martin; Fleckner, Uwe; Ziegler, Hendrik (Hrsg.) (2011): Handbuch der politischen Ikonografie. 2 Bände. München: C.H. Beck.

Wolf, Herta (Hrsg.) (2002): Paradigma Fotografie. Fotokritik am Ende des fotografischen Zeitalters. Frankfurt/Main: Suhrkamp.

Wolf, Herta (Hrsg.) (2003): Diskurse der Fotografie. Fotokritik am Ende des fotografischen Zeitalters. Frankfurt/Main: Suhrkamp. 



\title{
Der Film als historische Quelle
}

\author{
Wolfgang Koller
}

Während der Vorstellung [...] verließen viele Zuschauer fluchtartig das Lokal. ,Das ist ja nicht zum Aushalten', ertönte es hinter mir; und: "Wie darf man uns so etwas bieten!" (Siegfried Kracauer, Frankfurter Zeitung, 27.5.1930)

Am 23. Mai 1930 läuft in Berlin der Spielfilm „Westfront 1918“ an, der erste deutsche Tonfilm über den Ersten Weltkrieg (1914-1918). Auf neuartige Weise transportiert er Sinneseindrücke auf Ton- und Bildebene vom Kriegsgeschehen, vom Geknatter der Maschinengewehre bis zur Naheinstellung der Todesangst und des Sterbens. Die Regiearbeit von G. W. Pabst führt wegen seiner realitätsnahen und schonungslosen Schilderungen der Kriegsgräuel und der physischen und psychischen Zerstörung von Menschen zu heftigen Reaktionen. Kritiker empören sich über die unzumutbare Ausschlachtung der Grausamkeit und die vermittelte Antikriegsbotschaft - in ihren Augen ein Hohn für die soldatische Pflichterfüllung und Opferbereitschaft von Millionen von Frontsoldaten. Andere begrüßen, dass gerade die ausgereifte Technik des Filmmediums erst Krieg in seiner ganzen Unmenschlichkeit vor Augen führen und ein Umdenken in der Gesellschaft bewirken könne (vgl. Kester 2003: 147-160). Trotz oder gerade wegen der nervlichen Belastung, die „Westfront 1918“ dem Zuschauer offenkundig abverlangt, wird er einer der größten Kassenerfolge des Jahres. ${ }^{1}$ Es ist die Zeit, als um die Deutung des wenige Jahre zuvor verlorenen Ersten Weltkriegs mit rund zwei Millionen gefallenen Deutschen

\footnotetext{
1 Dies geht aus einer Umfrage der Fachzeitschrift Film-Kurier unter Kinobesitzern hervor (FilmKurier vom 23. Mai 1931).
} 
heftigste Auseinandersetzungen toben. Filme wie „Westfront 1918“ oder die USProduktion „Im Westen nichts Neues“ (im Original: „All Quiet on the Western Front“, Regie: Lewis Milestone) sieben Monate später ziehen den Sinn des Krieges und des Sterbens für das „höhere Ziel des Vaterlandes“ in Zweifel, während die Mehrzahl der zeitgenössischen Kriegsfilme das Heldentum der Frontkämpfer beschwört.

Die Weltkriegsfilme jener Jahre liefern ein eindrückliches Beispiel dafür, wie die Bilder, die über die Kinoleinwände flimmern und wie die Geschichten, die sie erzählen, die Menschen ihrer Zeit bewegen können. Sie geben zugleich der Nachwelt Auskunft, welche Fragen die Zeitgenossen sich stellen und welche Vorstellungen und Argumentationen kursieren. Filme ziehen Menschen seit Beginn des 20. Jahrhunderts in ihren Bann und erreichen schon früh ein Millionenpublikum. Bald wird dieses Medium kultisch verehrt und zur liebgewordenen Gewohnheit, zu einem imaginären Zufluchtsort vor den täglichen Sorgen, aber ebenso zum Austragungsort weltanschaulicher Grabenkämpfe. Filme bieten den Zuschauern Vorbilder und Gegenbilder dar, provozieren und bestätigen, klären ebenso auf wie sie manipulieren.

In der Geschichtswissenschaft ist heute weitgehend unbestritten, dass Filme eine wertvolle Quelle darstellen, um die Lebenswelten der Menschen im 20. und beginnenden 21. Jahrhundert zu erforschen. Bis dieses Medium allerdings als Untersuchungsgegenstand für Historiker breite Akzeptanz findet, vergehen Jahrzehnte. Dieser Prozess der Anerkennung von Filmen als historische Quellen lässt sich vor allem auf zwei grundlegende Fragen reduzieren, um die die Diskussionen kreisen. Eine betrifft ihre Aussagekraft: Sind Filme von geschichtswissenschaftlicher Bedeutung, bzw. worüber geben sie Auskunft? Die andere betrifft ihre Qualität als historische Quelle: Wie sind Filme zu untersuchen? Wie kann man über Filme schreiben? Die folgenden Seiten werden entlang dieser Fragen die Entwicklung der Filmhistoriographie aufzeigen und zentrale Forschungsfelder präsentieren.

\section{Abbilder der Wirklichkeit oder Illusionsfabrik?}

Als die Brüder Lumière 1895 ihre ersten Filme vorführen, sind es Aufnahmen von Arbeitern beim Verlassen der Lumière-Werke oder von der Ankunft eines Zuges auf dem Bahnhof in La Ciotat. Technisch ist es nun möglich - das wollen die beiden Filmpioniere verdeutlichen -, nicht nur wie in der Malerei oder der Fotografie Augenblicke einzufangen, sondern das Leben in seiner natürlichen Bewegtheit. Entsprechende Hoffnungen weckt das neue Medium, dass künftig die Wirklichkeit für die Nachwelt speicherbar sei. Die folgenden Jahrzehnte führen indes vor Augen, dass das filmische Potential, Illusionen zu schaffen, schier unendlich ist und letztlich dem fiktionalen Spielfilm zum Siegeszug verhilft. Ebenso groß scheint damals die Bereitschaft von Filmemachern, dieses Potential einzusetzen, um als 
real zu deklarieren, was kunstvoll arrangiert ist. Die anfänglichen Hoffnungen weichen der Ernüchterung: Auch „dokumentarische Filme“ bilden die Wirklichkeit nicht tatsächlich $\mathrm{ab}$, sondern lassen vielmehr authentisch wirkende Welten entstehen.

Dieser Umstand mag die Jahrzehnte währende Skepsis der Historikerzunft dem Film gegenüber erklären. Entziehen sich dokumentarische Filme doch einer klaren Einordnung im Sinne der klassischen Quellenbewertung: als Überrest, also einer nicht bewusst für spätere Generationen hergestellten Quelle, oder als bewusst an die Zeitgenossen und die Nachwelt gerichteten Traditionsquelle. Fiktionale Filme scheinen unter diesem Gesichtspunkt erst recht keinen Mehrwert in der Frage zu liefern, „wie es eigentlich gewesen“ ist (Leopold von Ranke) zu Zeiten ihrer Entstehung. ${ }^{2}$

Zur Einschätzung, dass Filme wertvolle neue Aussagen über die Gesellschaft liefern, gelangt als einer der ersten der Kritiker und Soziologe Siegfried Kracauer in seiner 1947 veröffentlichten Studie „From Caligari to Hitler“. Er durchleuchtet das Filmschaffen der Weimarer Republik auf kollektive „psychologische Grundmuster" der Deutschen und macht darin autoritäre Dispositionen ausfindig, die Hitler den Weg zur Macht erleichtert hätten (Kracauer 1947: 14). ${ }^{3}$ Kracauers Werk ist längst ein Klassiker, nicht aufgrund der umstrittenen und zweifelhaften Thesen, sondern als Inspirationsquelle für die Filmsoziologie und Filmhistoriographie. ${ }^{4}$ Filme liefern Informationen über die Gesellschaft, in der sie entstehen - oftmals mehr implizit als über den konkreten inhaltlichen Realitätsbezug. Das trifft auf fiktionale wie nichtfiktionale Produktionen $\mathrm{zu}$, auf Verfilmungen historischer Ereignisse wie das eingangs erwähnte Beispiel ebenso wie auf animierte Phantasiewelten.

\section{Cultural Turn}

Die Debatte um die Anerkennung des Films in der Historiographie setzt jedoch erst in den 1960er Jahren ernsthaft ein. Vorreiter ist hier die französische Geschichtswissenschaft. 1961 findet ein Beitrag des Filmessayisten Georges Sadoul über Filme als historische Quelle Eingang in den Sammelband „L'Histoire et ses méthodes“ (Samaran 1961). Wesentlich zur Etablierung trägt schließlich Marc Ferro bei. Ihm zufolge sind fiktionale wie nicht fiktionale Filme gleichermaßen bedeutsame Zeugnisse für Historiker, ist das Stattgefundene und das nicht Stattgefundene gleichermaßen Geschichte. Er begründet dies mit dem Argument, Filme

2 Zur Diskussion und Entwicklungsgeschichte des Films als historische Quelle sind unter anderem erschienen: Heiß 2006; Riederer 2003; Aurich 1995; Pithon 1995; Heß 1986.

3 Den wesentlich plausibleren Weg geht das kürzlich erschienene Buch von Kaes 2009. Auch Kaes folgt den Spuren des Unbewussten im Weimarer Kino, erklärt die Filme allerdings über die Verarbeitung der traumatischen Erfahrungen im Ersten Weltkrieg.

4 Die Filmsoziologin der ersten Stunde ist Emilie Altenloh mit ihrer Doktorarbeit 1913 über die Soziologie des Kinos (Altenloh 1914). 
erlaubten einen Blick unter die Oberfläche der Gesellschaft auf ihre „Überzeugungen, die Intentionen, das Imaginäre des Menschen“ (Ferro 21987: 254). ${ }^{5}$

Ferro wie eine zunehmende Zahl von Wissenschaftlern seiner Generation sind überzeugt, dass den bewegten Bildern zeitgenössische Mentalitäten und Wahrnehmungen eingeschrieben sind. ${ }^{6}$ An einem Film sind Drehbuchautoren, Produzenten, Regisseure, Kameraleute, Darsteller, Techniker und zahlreiche weitere Personen beteiligt. Aufgrund dieses kollektiven Herstellungsprozesses, aber auch, weil die Beteiligten zumeist angehalten sind, sich aus Absatzgründen am Geschmack der Mehrheit zu orientieren, können Filme repräsentative zeitgenössische Ausdrucksformen beinhalten. Darüber hinaus wirkt dieses Massenmedium durch seinen enormen Verbreitungsgrad wiederum in die Gesellschaft hinein. Es beeinflusst tagtäglich die Lebenswelt unzähliger Menschen. Bereits 1914 werden beispielsweise in Großbritannien 360 Millionen Karten in den landesweit 5.000 Kinos verkauft (vgl. Hiley 1999: 39-53). Das Inselkönigreich verfügt damals über die beste Infrastruktur, aber längst wird die Kinematographie auch auf dem europäischen Kontinent und in Nordamerika zum Massenphänomen. In den folgenden Jahrzehnten legen die Zuschauerzahlen noch deutlich zu, bis ab den späten 1950ern langsam das Fernsehen dem Kino Konkurrenz macht.

Die Forschung an Filmen als historischen Dokumenten lässt Rückschlüsse auf verbreitete Mentalitäten, Stereotypen oder Geschichtsbilder zu. Die Analyse des Entstehungsprozesses kann die medienpolitischen Machtverhältnisse und Motivlagen der Akteure aufdecken. ${ }^{7}$ Doch was ist mit dem Publikum? Kino steht beispielsweise für Abendunterhaltung vor der Leinwand. Darüber hinaus versorgen Fach- und Fanzeitschriften ebenso wie Tageszeitungen in ihren Filmrubriken eine millionenschwere Leserschaft, die das neue Programm studiert oder Anteil am Leben der Filmstars nimmt. Die Analyse der Kinokultur gibt also Aufschluss über Alltagsgewohnheiten und -vorlieben, über die Spuren, die die „laufenden Bilder“ in einer Gesellschaft hinterlassen.

Dass diese verschiedenen Aspekte seit den 1970er Jahren von Historikerseite vermehrt unter die Lupe genommen werden, verdanken wir einem grundlegenden Perspektivwechsel, der oft mit dem Schlagwort „Cultural Turn“ umschrieben wird. Das Blickfeld, das bis dahin die politische und die Sozialgeschichte umfasst, erweitert sich zunehmend um die Kultur- und Alltagsgeschichte. Das führt zu einer größeren Offenheit für neue, bisher vernachlässigte Quellen wie den Film. ${ }^{8}$ Das wissenschaftliche Hantieren mit Filmen setzt allerdings die Entwicklung einer dem Medium angemessenen historisch-kritischen Methode voraus.

\footnotetext{
${ }^{5} \mathrm{Zu}$ Ferros wichtigsten Arbeiten gehört Ferro 1977.

${ }^{6}$ Erwähnt sei hier nur Monaco 1973.

${ }^{7}$ Für die deutsche Filmproduktion im Nationalsozialismus erforscht dies beispielsweise Albrecht 1969.

${ }^{8}$ Zum kulturgeschichtlichen Wert von Filmen vgl. Kaes 1992.
} 


\section{Wie „übersetzt“ man einen Film?}

Die Nichtschriftlichkeit des Films stellt gewiss einen weiteren wesentlichen Grund für seine lange Vernachlässigung dar. Wie geht man quellenkritisch an einen Film heran? Wie kann ein Bewegtbild in Textform analysiert, also „übersetzt“ werden?

Dem schreibenden und für gewöhnlich lesenden Wissenschaftler bieten sich viele Möglichkeiten des Scheiterns. So erweist sich der Versuch, die Leinwandbilder vollständig zu beschreiben und zu analysieren, beispielsweise von der Farbe und dem Faltenwurf des Kleides der Protagonistin, der sich langsam veränderten Mimik und Gestik, der musikalischen Untermalung der Szene, den auf- und abtretenden Figuren im Hintergrund bis zum Wolkenspiel am Himmel als völlig unpraktikabel. Kein Text kann die Facetten eines Films in toto adäquat wiedergeben. Durch den Medientransfer und die erforderliche Dekodierung eines Zeichensystems in ein anderes schafft jede Rede über den Film wieder eine andere, eigene Geschichte. Zu akzeptieren, dass mit der Überführung des Films in einen Text notwendigerweise eine Reduktion und Verfremdung der analysierten Quelle einhergeht, ist also ebenso Voraussetzung wie ein profundes Wissen über die verschiedenen Wirkungsmechanismen des Mediums (vgl. Lagny; Sorlin 1991: 111128).

Filme weisen meist eine ähnliche Erzählstruktur wie Romane auf. Die festgelegte Anordnung von Bildern und Worten entscheidet darüber, welche Empfindungen, z.B. Spannung, ausgelöst und welche Kausalitäten hergestellt werden. Der Film arbeitet, ähnlich wie die Malerei oder Fotografie, mit ikonographischen Verdichtungen und schafft „Sinnbilder“, Metaphern und Symbole. Ähnlich wie im Theater agieren Schauspieler in unterschiedlichen Rollen in einer Figurenkonstellation. Filme weisen demnach Überschneidungen $\mathrm{zu}$ anderen Medien auf. Sie konstituieren aber auch eine ganz eigentümlich filmische Welt aus der Kameraperspektive, der Kamerabewegung im Raum und der Kombination der Kameraeinstellungen - im Wesentlichen umschrieben durch die Begriffe „Mise en Scène“ und „Montage“. Aus diesen Grundprinzipien und Techniken, wie Filme ihre Inhalte vermitteln, lassen sich methodische Grundlagen der Filmanalyse ableiten. Die Geschichtswissenschaft profitiert hier enorm von den Erkenntnissen anderer Disziplinen, insbesondere der Semiotik, der Narratologie und der Film- und Medienwissenschaft. ${ }^{9}$

Der Blick über den historiographisch-disziplinären Tellerrand ist auch hilfreich, wenn es um methodische Ansätze zur Untersuchung des filmischen Kontextes geht. Dies betrifft die Produktionsgeschichte, die Einflussfaktoren für die Filmentstehung, aber auch ihre zeitgenössische Aufnahme durch die Presse und das Publikum. Presserezensionen sind leider oftmals die einzigen Relikte, die heute noch ermöglichen, die einstige Wirkung beim Publikum einzuschätzen. In

${ }^{9}$ Zu den zentralen Werken über den Film zählen: Wollen 1972; Metz 1973; Branigan 1984; Bordwell 1985; Deleuze 1989; Deleuze 1991; Eder 2008. Eine Einführung in die Filmanalyse geben u.a.: Kanzog 2001; Bordwell; Thompson 2003; Faulstich 22008; Monaco 2009. 
Fragen der Kontextuierung erweisen sich besonders die Erkenntnisse der poststrukturalistischen Literatur- und Medienwissenschaft als gewinnbringend und inspirierend für die Herausbildung einer historiographischen Filmforschung. ${ }^{10}$ Filme werden angeregt und beeinflusst von anderen Filmen, literarischen Werken, Deutungsmustern der Vergangenheit und der zeitgenössischen Welt, von ökonomischen und künstlerischen Motiven. Sie sind an finanzielle und technischindustrielle Voraussetzungen geknüpft. Und sie hinterlassen ihrerseits Spuren in anderen Medienerzeugnissen oder im öffentlichen Diskurs.

Ein Beispiel: Der berüchtigte antisemitische Spielfilm ,Jud Süß“ (Regie: Veit Harlan, 1940) entstand im Auftrag des NS-Propagandaministers Josef Goebbels im Zuge der verschärften medialen Mobilmachung gegen Juden - man denke nur an die zeitgleich anlaufende, ebenso berüchtigte „dokumentarische“ Produktion von Fritz Hippler namens „Der ewige Jude“. ,JJud Süß“ war aber auch eine späte Reaktion der Nazis auf den 1934 von deutschen Exilanten in Großbritannien gedrehten Spielfilm „Jew Süss“ (Regie: Lothar Mendes). Beide Filme gehen auf die historisch verbürgte Person Josef Süß Oppenheimer (1698-1738) zurück. Aus einer jüdischen Kaufmannsfamilie stammend stieg er bis zum einflussreichen Finanzrat des Herzogs von Württemberg auf, was für einen Juden der damaligen Zeit einzigartig war. Spannungen zwischen dem absolutistisch herrschenden Landesfürsten und den Landständen sowie aufgestaute Ressentiments gegen den Emporkömmling entluden sich nach dem plötzlichen Tod des Herzogs an Süß Oppenheimer, der unter Vorwürfen wie Bestechung und Jungfrauenschändung verhaftet und hingerichtet wurde. Sein Schicksal wurde mehrfach literarisch verarbeitet, allerdings auf sehr unterschiedliche Weise. Erste antisemitische Schriften über den Fall erschienen noch im Jahr der Hinrichtung. Bekannt wurde dann Wilhelm Hauffs Novelle „Jud Süß“ aus dem Jahr 1868, die sich auf diese Rezeptionstradition stützt. Einen völlig anderen Akzent setzte Lion Feuchtwanger mit seinem gleichnamigen Roman von 1925. Zeichnet die eine Seite das Bild des habgierigen Ränkeschmieds, begreift ihn Feuchtwanger als Sündenbock. Feuchtwangers Roman inspirierte Lothar Mendes zur ,Jew Süss“-Produktion von 1934, der damit indirekt auch die Judenverfolgung unter den Nazis anklagt. Goebbels' Replik von 1940 greift hingegen wieder tief in die Kiste überlieferter Schmähungen und transferierte damit auch die antisemitische Geschichte Süß Oppenheimers auf die Leinwand (vgl. Hollstein 1971).

\footnotetext{
10 Zur Rezeptionsforschung vgl. Turner 1998; Moores 1990. Mit intertextuellen Aspekten beschäftigt sich Bennett; Woollacott 1987. Einen guten Überblick über die verschiedenen interdisziplinären Ansätze der Filmanalyse gibt Winter 1992. Auf Seiten der geschichtswissenschaftlichen Filmanalyse sind insbesondere zu nennen: Elsaesser; Buckland 2002; Korte 1999; Bock 1997; Lagny 1992; Staiger 1992.
} 


\section{Vom einzelnen Filmwerk zum Mainstream-Filmprogramm}

An den „Jud Süß“-Verfilmungen zeigt sich exemplarisch die intertextuelle und intermediale Verflechtung von zwei Kinoproduktionen. Das Problem solcher Einzelstudien ist aber, dass sie nur geringe Aussagekraft für das Kino einer Zeit und seine gesellschaftlichen Einprägungen haben. Steht in früheren filmhistorischen Arbeiten oftmals ein Kanon meist künstlerisch bedeutsamer oder häufig zitierter Werke im Vordergrund, oder durchleuchten Wissenschaftler wie Marc Ferro (vgl. Ferro 1987) Filme, die enthüllen sollen, was die offizielle und staatlich protegierte Geschichtsschreibung verdeckt, fokussieren neuere kulturwissenschaftliche Ansätze hingegen gerade die breite Masse der Standardware. Die Populärkultur wird zunehmend zum Untersuchungsgegenstand. ${ }^{11}$ Im Gegensatz zu den subversiven Ausnahmeproduktionen, Propagandamachwerken oder künstlerischen Highlights wandelt das Gros der Filmschaffenden auf den ausgetretenen Pfaden von Klischees und Wertekonventionen. Die Durchschnittsfilme werden oft in Serie gefertigt. Sie orientieren sich am Bekannten, an kommerziell bewähren Erfolgsrezepten, beliebten Genremustern und Themen. ${ }^{12}$

Genres entstehen, weil sie auf eine Art und Weise wiederholen, was besonders nachgefragt wird. Sie sind das Ergebnis eines Wechselspiels aus kreativen Impulsen, Produktion nach Schema und Reaktion an den Kinokassen. Für die Filmanalyse ist hier vor allem der kinematographische und gesellschaftliche Zusammenhang interessant. Warum ist ein Thema oder Genre zu einer bestimmten Zeit in einer bestimmten Gesellschaft so beliebt? Ein Beispiel aus dem TVBereich: In den 1960er Jahren kommen Science-Fiction-Serien wie „Raumpatrouille“ (Deutschland) und „Star Trek“ (USA) auf. Aufschlussreich ist schon der Blick auf Modegeschmack, Gesten oder Rollenverhalten, die mittlerweile in die Jahre gekommen und insofern ,historisierbar“" sind. Dies an einzelnen Folgen zu erörtern wäre auch ein praktisches Anwendungsgebiet für $\mathrm{DaF}$ bereits in den unteren Lernstufen. In höheren Niveaus könnten sich weitergehende Fragen anschließen, z.B. nach den zum Ausdruck kommenden damaligen Zukunftserwartungen in der westlichen Welt angesichts des wahrgenommenen Fortschritts, etwa des Apollo-Raumfahrtprojekts, des Sprungs in der Waffentechnik durch die Erfindung der Kernwaffen oder der Entwicklung des Computers.

Mainstream-Filme lassen aufgrund ihrer Vielzahl und ihres Zuspruchs beim Publikum Rückschlüsse sowohl auf landläufige Einstellungen und populäres Gedankengut wie auf mehrheitliche Sehgewohnheiten zu. Je nach Art des Zugangs fördern Untersuchungen Neues zu Tage, beispielsweise über das Filmstar-Phänomen, die in Kino und Fernsehen favorisierten Eigenschaften und Stereotypen von Filmhelden oder über die Ausformungen des Persönlichkeitskults im Mediengeschäft (vgl. Dyer 1979; Ascheid 2003). Ein bedeutendes Untersuchungsfeld

\footnotetext{
11 Einen solchen Ansatz wählt beispielsweise Stiasny 2009. Darin untersucht der Autor, welche Spuren der Erste Weltkrieg im populären Kino der Weimarer Republik hinterlassen hat.

12 Zu Genreaspekten vgl. Moine 2005; Koebner 2003; Seeßlen 1995; Landy 1991.
} 
markieren die Geschlechtervorstellungen, die Filme transportieren. Die GenderForschung hat auf diesem Gebiet interessante Erkenntnisse z.B. zur männlich dominierten Wahrnehmungsperspektive in Filmen hervorgebracht (Kaplan 1997; Mulvey 1985). Zunehmend stehen hier geschlechtlich ebenso wie ethnisch und sozial konstruierte Differenzen und Identitätsentwürfe im Fokus der Studien. ${ }^{13}$ Wie entwerfen Filme Idealbilder von Männlichkeit und Weiblichkeit? So wiederholt sich z.B. im Western häufig das Handlungsschema, dass der männliche Held als Einzelkämpfer die Grenze der Zivilisation überschreitet, räumlich wie im sozialen Gefüge, um letztlich seine Männlichkeit neu zu beweisen (vgl. Weidinger 2006).

Gegenstand vieler historiographischer Filmuntersuchungen ist die Konstruktion kollektiver, meist nationaler Identitäten und Alteritäten. ${ }^{14}$ Überschneidungen gibt es hier vor allem zum Forschungsfeld der historischen Mythen, Geschichtsdeutungen und Erinnerungskulturen. ${ }^{15}$ Historienfilme beeinflussen allein schon durch die Breitenwirkung des Mediums die kollektiven Geschichtsbilder und Erinnerungskulturen in Gesellschaften. Dieser Umstand macht sie für die Forschung so interessant. Was sagen beispielsweise Spielfilme wie „Der Name der Rose“ (Regie: Jean-Jacques Annaud, 1986) über unser Bild vom Mittelalter am Ende des 20. Jahrhunderts? Kinogroßproduktionen früherer Jahrzehnte setzen noch eher die höfische Kultur und den ritterlichen Edelmut in Szene. Hier sehen wir hingegen, wie Menschen in Schmutz und Elend vegetieren. Das Mittelalter erscheint uns als eine von Entbehrung, religiösem Fanatismus und Aberglaube gebeutelte Zeit.

Bedeutende Arbeiten sind in den letzten Jahrzehnten auch zur Geschichte der ökonomischen und organisatorisch-rechtlichen Rahmenbedingungen des Kinos entstanden. Dies betrifft die Filmwirtschaft/Filmindustrie, Konzernstrukturen, Studios und ihre Finanzierung. Oftmals nicht davon zu trennen sind die häufigen politischen Interventionen in das Filmwesen - ein Bereich, der verhältnismäßig gut aufgearbeitet ist. ${ }^{16}$ Ähnliches gilt für die staatliche Filmgesetzgebung und Zensurpolitik. ${ }^{17}$

\section{Quo vadis Filmgeschichte?}

Die Filmgeschichtsschreibung ist heute stark interdisziplinär ausgerichtet und unter vielfältigen Fragestellungen für die Geschichtswissenschaft von Nutzen. So ent-

\footnotetext{
13 Unter den zahlreichen Filmstudien der Genderforschung vgl. unter anderem Babington; Davies; Powrie 2004. Benshoff; Griffin 2009.

$14 \mathrm{Zu}$ diesem Forschungsgegenstand sind insbesondere zu nennen: McLaughlin 2010; Nagl 2009; Rother 1998.

15 Hierzu sind erschienen: Koller (in Vorbereitung); Braun 2010; Noack 2010; Erll; Wodianka 2008; Slanička 2007; Chiari; Rogg; Schmidt 2003; Grindon 1994.

${ }^{16}$ Bezogen auf Deutschland sind besonders erwähnenswert: Vande; Welch 2007; Hoffmann 1996; Rentschler 1996; Drewniak 1987; Spiker 1975.

17 Zur Filmzensur vgl. Sanders 2002; Robertson 1985.
} 
stehen zunehmend alltags- und mikrogeschichtliche wie auch sozialhistorische Studien über kulturelle Formen und Praktiken der Kinematographie. Das Aufkommen der „Lichtspielhäuser“ verändert Anfang des 20. Jahrhunderts das Gesicht der Städte und den Lebensrhythmus ihrer Bewohner. ${ }^{18}$ Filmvorführungen in den 1920er oder 1930er Jahren haben mitunter Showcharakter, brisante Themen führen zu Ausnahmezuständen und Protestaktionen im Kinosaal. ${ }^{19}$ Das neue Medium wird zu einem Faktor des öffentlichen Lebens. ${ }^{20}$ Es dringt, in manchen Fällen mittels massiver staatlicher Förderung, bis zur Jahrhundertmitte mehr und mehr in die Nischen des Alltags ein und erfasst immer größere Bevölkerungsteile (vgl. Stahr 2001).

Gerade die historiographisch so interessante Nahtstelle zwischen dem Unterhaltungs- und Informationsprodukt Film und der zeitgenössischen Gesellschaft ist aber oft nur schwer sichtbar zu machen. Besonders für die erste Hälfte des 20. Jahrhunderts stellt sich das Problem, wie aufgrund der schlechten Quellensituation die Verbreitungsräume von Filmen oder die Publikumsstrukturen rekonstruierbar sind. Über solche Studien ließe sich die gesellschaftliche Relevanz differenzierter darstellen: beispielsweise, ob es bei einem Genre oder behandelten Thema lokale, regionale, nationale Präferenzen oder Übereinstimmungen gab, ob es stärkeren Anklang unter Männern oder Frauen, im Arbeiter- oder bürgerlichen Milieu fand. Nachweise über häufige Reaktionen und Geschmacksurteile erlaubten wiederum konkretere Rückschlüsse auf die Aneignung von Filminhalten. Erschwerend für Untersuchungen über diesen Zeitraum kommt hinzu, dass auch ein beachtlicher Teil der Filme selbst als verschollen gilt. Wer sich der Erforschung der Anfangsjahre des Films oder des Zeitalters der Weltkriege verschreibt, begibt sich auf ein schwieriges Terrain.

Die nächsten Jahre könnten hier für Historiker einen Zuwachs der Materialbasis bringen. Zumindest besteht Anlass zur Hoffnung, bedenkt man die Möglichkeiten der elektronischen Massenspeicherung und Volltextdigitalisierung von historischen Tageszeitungen und Fachzeitschriften, die damals beispielsweise über örtliche Filmprogramme berichteten und eine Auswahl der Produktionen rezensierten. Viel versprechende Projekte existieren bereits jetzt, etwa die Internetplattform zur Informations- und Dokumentensammlung über verschollene Filme namens Lost Films. ${ }^{21}$ Weit gediehen sind in den letzten Jahren auch die Onlineportale, zumeist der nationalen Filminstitute, die über die Filmdaten hinaus teilweise auch zeitgenössische Materialien wie Zensurprotokolle, Presserezensionen und Informationsbroschüren zum Filmstart zur Verfügung stellen. ${ }^{22}$

\footnotetext{
18 Über den frühen Kinoboom ist erschienen: Hiley 2002. Mit lokaler Kinokultur befasst sich Maier 2009.

${ }^{19}$ Hierzu ist kürzlich erschienen: Nowak 2010.

${ }^{20}$ Besonders interessant ist hierzu Müller; Segeberg 2008.

${ }^{21}$ Lost Films: http://www.lost-films.eu/

${ }^{22}$ International ausgerichtet sind die Filmdatenbanken Internet Movie Database (www.imdb.com, frei zugänglich), und Treasures from the Film Archives, eine zugangsbeschränkte Datenbank der
} 
Es ist zu hoffen, dass eine bessere Erschließung von Filmzeitschriften künftig auch zu mehr Studien führt, die sich an der historischen Wirklichkeit der Kinokultur orientieren. Nach wie vor wird allzu häufig Filmgeschichte von ihrem Produktions- und nicht ihrem Aufführungsort her gedacht. Sich hier nur auf das nationale Filmschaffen zu konzentrieren, verzerrt aber die historische Realität. Bereits in den 1920er Jahren kommen in Frankreich und Großbritannien mehr USamerikanische Filme in die Kinos als heimische Produktionen. Auch in Deutschland ist die Situation in dieser Zeit nicht grundlegend anders. Ein stark internationales Filmangebot ist für Europa wie große Teile der Welt fast zu allen Zeiten die Regel und nicht die Ausnahme. Warum untersuchen Filmhistoriker beispielsweise immer noch den deutschen Film anstatt der Kinokultur in Deutschland mit all ihren Facetten, vom Heimatfilm bis hin zum Hollywood-Western? Warum gibt es so wenige transnationale Studien, die den filmischen Kulturtransfer über die Landesgrenzen hinaus betrachten oder Vergleiche zwischen Regionen und Ländern vornehmen? Ein Grund liegt in der meist staatlichen bzw. öffentlich organisierten Sammlungspolitik, so dass Wissenschaftler in Fragen der Filmsichtung und Dokumentenrecherche vor besonderen Herausforderungen stehen. Doch die positiven Ansätze der letzten Jahre sind unverkennbar. Sie geben Anlass zur Hoffnung, dass Historiker der gesellschaftlichen Bedeutung, die der Film seit dem frühen 20. Jahrhundert hat, Stück für Stück in seinen vielfältigen Ausprägungen näher kommen.

\section{Literatur}

Albrecht, Gerd (1969): Nationalsozialistische Filmpolitik. Stuttgart: Enke.

Altenloh, Emilie (1914): Zur Soziologie des Kino. Die Kino-Unternehmung und die sozialen Schichten ihrer Besucher. Jena: Diederichs.

Ascheid, Antje (2003): Hitler's Heroines. Stardom and Womenhood in Nazi Germany. Philadelphia: Temple University Press.

Aurich, Rolf (1995): Wirklichkeit ist überall. Zum historischen Quellenwert von Spiel- und Dokumentarfilmen. In: Wilharm, Irmgard (Hrsg.) (1995): Geschichte in Bildern. Von der Miniatur bis zum Film als historischer Quelle. Pfaffenweiler: Centaurus, 112-128.

Babington, Bruce; Davies, Ann; Powrie, Phil (Hrsg.) (2004): The Trouble with Men: Masculinities in European and Hollywood Cinema. London: Wallflower Press.

International Federation of Film Archives (FIAF) zu Stummfilmen. Zu französischen Filmen sind die Datenbanken der Archives françaises du film (http://www.cnc-aff.fr) und der Bibliothèque du film (http://www.bifi.fr) empfehlenswert, zu deutschen Filmen die zentrale Internetplattform filmportal.de sowie die Materialsammlung des Deutschen Filminstituts (http://www.deutschesfilminstitut.de), zu britischen Filmen die Datenbanken des British Film Institute (http://ftvdb.bfi.org.uk und http://www.screenonline.org.uk). 
Bennett, Tony; Woollacott, Janet (1987): Bond and Beyond: The Political Career of a Popular Hero. Basingstoke u.a.: Palgrave Macmillan.

Benshoff, Harry; Griffin, Sean (2009 [2003]): America on Film: Representing Race, Class, Gender, and Sexuality at the Movies. Oxford: Wiley-Blackwell.

Bock, Hans-Michael (Hrsg.) (1997): Recherche: Film. Quellen und Methoden der Filmforschung. München: Edition Text + Kritik.

Bordwell, David (1985): Narration in the Fiction Film. London: Methuen.

Bordwell, David; Thompson, Kristin (2003): Film Art: An Introduction. New York: McGraw-Hill.

Branigan, Edward (1984): Point of View in the Cinema. A Theory of Narration and Subjectivity in Classical Film. Berlin u.a.: Mouton.

Braun, Brigitte (2010): Mit FRIDERICUS REX gegen Franzosen und Belgier. Nationales Kino im Ruhrkampf 1923, In: Filmblatt 42, 67-85.

Chiari, Bernhard; Rogg, Matthias; Schmidt, Wolfgang (Hrsg.) (2003): Krieg und Militär im Film des 20. Jahrbunderts. München: Oldenbourg.

Deleuze, Gilles (1989): Das Bewegungs-Bild. Kino 1. Frankfurt/Main: Suhrkamp.

Deleuze, Gilles (1991): Das Zeit-Bild. Kino 2. Frankfurt/Main: Suhrkamp.

Drewniak, Boguslaw (1987): Der deutsche Film 1938-1945: Ein Gesamtüberblick. Düsseldorf: Droste.

Dyer, Richard (1979): Stars. London: British Film Institute.

Eder, Jens (2008): Die Figur im Film. Grundlagen der Figurenanalyse. Marburg: Schüren.

Elsaesser, Thomas; Buckland, Warren (2002): Studying Contemporary American Film. A Guide to Movie Analysis. London u.a.: Arnold.

Erll, Astrid; Wodianka, Stephanie (Hrsg.) (2008): Film und kulturelle Erinnerung. Plurimediale Konstellationen. Berlin: de Gruyter.

Faulstich, Werner (22008): Grundkurs Filmanalyse. Paderborn: Fink.

Ferro, Marc (1977): Cinéma et Histoire. Paris: Hachette.

Ferro, Marc (21987): Der Film als „Gegenanalyse“ der Gesellschaft. In: Honegger, Claudia (Hrsg.): Schrift und Materie der Geschichte: Vorschläge zur systematischen Aneignung historischer Prozesse. Frankfurt/Main: Suhrkamp, 247-271.

Grindon, Leger (1994): Shadows of the Past: Studies in the Historical Fiction Film. Philadelphia: Temple University Press.

Heiß, Gernot (2006): Film als Quelle. In: Wiener Zeitschrift zur Geschichte der Neuzeit $6 / 2,99-108$. 
Heß, Klaus-Peter (1986): Film und Geschichte. Kritische Einführung und Literaturüberblick. In: Film Theory: Bibliographic Information and Newsletter 13, 196-226.

Hiley, Nicholas (1999): „Let's go to the pictures“. The British Cinema and Audience in the 1920s and 1930s. In: Journal of Popular British Cinema 2, 39-53.

Hiley, Nicholas (2002): „Nothing More than a ,Craze“،. Cinema Building in Britain from 1909 to 1914. In: Higson, Andrew (Hrsg.): Young and Innocent? The Cinema in Britain 1896-1930. Exeter: University of Exeter Press, 111-127.

Hollstein, Dorothea (1971): Antisemitische Filmpropaganda. Die Darstellung des Juden im nationalsozialistischen Spielfilm. München-Pullach: Verl. Dokumentation.

Kaes, Anton (1992): Filmgeschichte als Kulturgeschichte. Reflexionen zum Kino der Weimarer Republik. In: Jung, Uli; Schatzberg, Walter: Filmkultur zur Zeit der Weimarer Republik. München u.a.: Saur, 54-64.

Kaes, Anton (2009): Shell Shock Cinema: Weimar Culture and the Wounds of War. Princeton: Princeton University Press.

Kanzog, Klaus (2001): Grundkurs Filmrhetorik. München: Diskurs-Film-Verlag Schaudig \& Ledig.

Kaplan, Elizabeth Ann (1997): Looking for the Other: Feminism, Film, and the Imperial Gaze. London u.a.: Routledge.

Kester, Bernadette (2003): Film Front Weimar. Representations of the First World War in German Films of the Weimar Period (1919-1933). Amsterdam: Amsterdam University Press.

Koller, Wolfgang (in Vorbereitung): Die Erinnerungen an die Napoleonischen Kriege im europäischen Film. Paderborn: Schöningh.

Korte, Helmut (1999): Einführung in die systematische Filmanalyse. Berlin: Erich Schmidt.

Kracauer, Siegfried (1947): From Caligari to Hitler. Princeton: Oxford University Press.

Lagny, Michèle (1992): De l'histoire du cinéma: méthode historique et histoire du cinéma. Paris: Armand Colin.

Lagny, Michèle; Sorlin, Pierre (1991): Zwei Historiker nach einem Film: ratlos. In: Rainer Rother (Hrsg.): Bilder schreiben Geschichte: Der Historiker im Kino. Berlin: Wagenbach, 111-128.

Landy, Marcia (1991): British Genres. Cinema and Society, 1930-1960. Princeton: Princeton University Press.

Maier, Margit (2009): Das Geschäft mit den Träumen: Kinokultur in Würz̧burg. Würzburg: Königshausen \& Neumann. 
McLaughlin, Noah (2010): French War Films and National Identity. Amherst (NY): Cambria Press.

Metz, Christian (1973): Sprache und Film. Frankfurt/Main: Athenäum.

Moine, Raphaëlle (2005): Le cinéma français face aux genres. Paris: AFRHC.

Monaco, James (2009): Film verstehen: Kunst, Technik, Sprache, Geschichte und Theorie des Films und der Neuen Medien, deutsche Fassung hrsg. von Hans-Michael Bock. Reinbek bei Hamburg: Rowohlt.

Monaco, Paul (1973): Cinema and Society. France and Germany During the Twenties. New York: Elsevier.

Moores, Shaun (1990): Texts, Readers and Contexts of Reading: Developments in the Study of Media Audiences. In: Media, Culture and Society 12/1, 9-29.

Müller, Corinna; Segeberg, Harro (Hrsg.) (2008): Kinoöffentlichkeit (1895-1920). Entstehung, Etablierung, Differenzierung. Marburg: Schüren.

Mulvey, Laura (1985): Visual Pleasure and Narrative Cinema. In: Nichols, Bill (Hrsg.): Movies and Methods. Berkeley u.a.: University of California Press.

Nagl, Tobias (2009): Die unheimliche Maschine: Rasse und Repräsentation im Weimarer Kino. München: Edition Text + Kritik.

Noack, Bettina (2010): Gedächtnis in Bewegung: Die Erinnerung an Weltkrieg und Holocaust im Kino. München: Fink.

Nowak, Kai (2010): Kinemaklasmus. Protestartikulation im Kino. In: Bösch, Frank; Schmidt, Patrick (Hrsg.): Medialisierte Ereignisse. Performanz, Inszenierung und Medien seit dem 18. Jahrbundert. Frankfurt/Main u.a.: Campus-Verlag, 179197.

Pithon, Rémy (1995): Cinéma et histoire: Bilan historiographique. In: Vingtième siècle. Revue d'histoire 46, 5-13.

Rentschler, Eric (1996): The Ministry of Illusion. Nazi Cinema and Its Afterlife. Cambridge (Mass.) u.a.: Harvard University Press.

Riederer, Günter (2003): Den Bilderschatz heben - vom Verhältnis zwischen Geschichtswissenschaft und Film. In: Tel Aviver Jabrbuch für deutsche Geschichte 31, 15-40.

Robertson, James C. (1985): The British Board of Film Censors: Film Censorship in Britain, 1896-1950. London u.a.: Croom Helm.

Rother, Rainer (Hrsg.) (1998): Mythen der Nationen: Völker im Film. München: Koehler \& Amelang.

Samaran, Charles (Hrsg.) (1961): L'Histoire et ses méthodes. Paris: Gallimard. 
Sanders, Lise Shapiro (2002): Indecent Incentives to Vice. Regulating Films and Audience Behaviour from the 1890s to the 1910s. In: Higson, Andrew (Hrsg.): Young and Innocent? The Cinema in Britain 1896-1930. Exeter: University of Exeter Press, 97-110.

Seeßlen, Georg (1995): Western. Geschichte und Mythologie des Westernfilms. Marburg: Schüren.

Slanička, Simona (Hrsg.) (2007): Antike und Mittelalter im Film. Konstruktion Dokumentation - Projektion. Köln u.a.: Böhlau.

Spiker, Jürgen (1975): Film und Kapital. Der Weg der deutschen Filmwirtschaft zum nationalsozialistischen Einheitskonzern. Berlin: Spiess.

Stahr, Gerhard (2001): Volksgemeinschaft vor der Leinwand? Der nationalsozialistische Film und sein Publikum. Berlin: Hans Theissen.

Staiger, Janet (1992): Interpreting Films - Studies in the Historical Reception of American Cinema. Princeton: Princeton University Press.

Stiasny, Philipp (2009): Das Kino und der Krieg: Deutschland 1914-1929. München: Edition Text + Kritik.

Turner, Graeme (1998): Film as Social Practise. London u.a.: Routledge.

Vande, Roel; Welch, David (Hrsg.) (2007): Cinema and the Swastika: The International Expansion of Third Reich Cinema. Basingstoke: Palgrave Macmillan.

Weidinger, Martin (2006): Nationale Mythen - Männliche Helden. Politik und Geschlecht im amerikanischen Western. Frankfurt/Main: Campus.

Winter, Rainer (1992): Filmsoziologie. Eine Einfübrung in das Verbältnis von Film, Kultur und Gesellschaft. München: Quintessenz.

Wollen, Peter (1969): Signs and Meaning in the Cinema. London: Secker \& Warburg. 


\title{
Zeitgeschichtliche Filmszenen für den DaF- Unterricht - Praktische Beispiele und Erfahrungen
}

\author{
Jens Grimstein
}

\section{Einleitung}

Es hat lange gedauert, bis sich der Film als historische Quelle für kulturgeschichtliche Forschungsinteressen hat etablieren können. ${ }^{1}$ Die Zweifel seitens einer sich vor allem als Textwissenschaft verstehenden Geschichtswissenschaft gegen die bewegten Bilder hielten lange an. Wenn überhaupt ein filmisches Medium zu Forschungszwecken taugte, dann war es aus nahe liegenden Gründen der Dokumentarfilm. Dass allerdings auch dieser filmsprachlichen Inszenierungskonventionen (und Manipulationsversuchungen) unterlag, wurde zu selten in Betracht gezogen und stattdessen sein dokumentarischer Wert im realitätsgetreuen Abbilden historischer Ereignisse hervorgehoben. Ihm gegenüber zählte man den typischerweise abendfüllenden, durchschnittlich ein- bis zweistündigen fiktionalen Spielfilm zur Unterhaltung und übersah dabei sein Vermögen als Vermittler diskursiver Praktiken.

Dies hat sich in den vergangenen Jahrzehnten geändert. Mittlerweile erfährt der Spielfilm als historische Quelle eine große Aufmerksamkeit in der Kultur- und Alltagsgeschichte. Er eröffnet Einblicke in die Gewohnheiten, Vorlieben, Denk-

\footnotetext{
${ }^{1}$ Vgl. Abraham; Kepser 2009: 168f. Da grundsätzlich vorausgesetzt werden darf, was ein Film ist, soll an dieser Stelle auf ein Standardwerk zur Filmtheorie und -praxis verwiesen werden. Vgl. auch Hickethier 2007: 22ff.
} 
und Handlungsweisen von Menschen einer sowohl vergangenen als auch jüngeren Zeit. ${ }^{2}$ Der Einsatz von Filmmaterial im praxisnahen „Deutsch als Fremdsprache“ (DaF)-Unterricht gilt heutzutage ebenfalls als normal, auch wenn methodischfilmdidaktische Theoriewerke hierfür bislang noch fehlen. ${ }^{3}$ Der Film darf somit als „fiktionales Leitmedium“ (Abraham; Kepser 2009: 167) gelten, der in einer aktuell bildwütigen Zeit enorme Aufmerksamkeit auf sich zieht. Unsere Weltwahrnehmung orientiert sich demnach maßgeblich an den bewegten und hörbaren Bildern. ${ }^{4}$ Dies gilt auch für geschichtliche Filmthemen, die von einem großen Publikum rezipiert werden. ${ }^{5}$ Ein offenkundig gesellschaftliches Bedürfnis nach Geschichte scheint im Film auf fruchtbaren Boden zu fallen.

Filme sind multimediale Werke, die verschiedene technische und geistige Komponenten in sich vereinigen. ${ }^{6}$ Als Zuschauer nimmt man den Film als darstellendes Kunstwerk wahr. Er zeigt in rascher Folge - der englische Begriff „pictures“ erinnert daran - Einzelbilder, die technisch so schnell vor unserem Auge ablaufen, dass wir sie als bewegt wahrnehmen. Auf diese Weise wird in Einstellungen, Szenen und Sequenzen der Film erzählt. ${ }^{7}$ Diese kinematographische Grundlage ist von großer Wichtigkeit, denn über die (geschnittenen) Bilder nehmen wir im Einklang mit Dialogen der Protagonisten, Hintergrundgeräuschen und ggf. Musik die unterschiedlichen Informationen auf, um den Film zu verstehen. Im Vergleich zur Schriftsprache ist dies die Symbolebene des Films, die im Begriff der Bildästhetik oder „Bildsprache“ (und deren Narration) ihre Entsprechung findet.

In einem sich vor allem als Sprach- und weniger als Landeskundeunterricht verstehenden Fremdsprachenunterricht besetzt die Bildsprache auf diese Weise eine Position, die es erlaubt, zwei Kategorien in Aufgaben für DaF-Lerner zusammenzuführen: Die Ebene der Bildsprache und die Ebene der Sprachfertigkeit, in diesem Fall die der deutschen Sprache. ${ }^{8}$ Beide Kategorien produzieren Zeichen, die ihrerseits mit unterschiedlichen Mitteln Mitteilungen über die Wirklichkeit innerhalb eines kommunikativen und medialen Bezugsrahmens machen. Konkret für die Arbeit mit Filmszenen als historische Queller für den DaF-Unterricht bedeutet dies, dass eine Filmszene ein bestimmtes Aussagepotential über einen historischen

2 Zur Kulturgeschichte des Films am Beispiel der Darstellung der Napoleonischen Kriege im Film des frühen 20. Jahrhunderts vgl. exemplarisch Koller (in Vorbereitung). Es beschäftigen sich außerdem mit Geschichte im Film: Wedel 2011; Gehrke 2011; Schanze 1996; Kotulla 1964.

3 Vgl. Abraham; Kepser 2009: 167.

${ }^{4}$ Noch offen ist, inwiefern die neue Videospielfilmkultur, die intensiv das aktive Einbringen des Nutzers fordert und fördert, einen neuen Einfluss auf die Sehgewohnheiten jüngerer Generationen hat.

5 Es ist davon auszugehen, dass Film und Fernsehen in der Rezeption historischer Stoffe weit vor Textquellen liegen. Die Zuschauerzahlen für historische Filme wie „Gladiator“ (USA 2000, Regie: Ridley Scott) oder „Der Untergang“ (BRD 2004, Regie: Oliver Hirschbiegel), aber auch die erfolgreichen Geschichtsserien des Senders ZDF belegen dies.

${ }^{6}$ Damit sind z.B. Filmideen, das Schreiben eines Drehbuchs, aber auch alle technischen Hilfsmittel von der Kamera über die Requisiten bis zum Schnitt gemeint.

7 Vgl. Hickethier 2007: 52.

${ }^{8}$ Nicht berücksichtigt werden hier filmsprachliche Analyse- und Arbeitskriterien, wie z.B. die Montage, der Schnitt usw., die man analog zur Schriftsprache auch „Filmgrammatik“ nennt. 
Abschnitt liefert. Dieses Aussagepotential wiederum kann angewandt werden in fremdsprachenspezifischen Übungen für Deutschlerner, indem es innerhalb einer Übung verbalisiert wird und dadurch zur eigenen Produktion von sprachlichen Erzeugnissen motiviert. Die Filmszene kann in dieser Hinsicht als eine „Verbildlichung“ kulturell-sozialer Gesellschaftsbedingungen didaktisch in einer Übung ihre Anwendung finden und hierbei die Sprachkompetenz des Lerners fördern. Zwischen der Symbolsprache des Bildes mit Bezug auf historische Begebenheiten und der Zielsprache ergibt sich so eine Synthese, indem die Bildsprache und die historische Begebenheit im fremdsprachlichen Medium ihren Ausdruck finden. Sowohl ein mehrdeutiges Leseverstehen (im Sinne eines Dechiffrierens des Films und des Verstehens von Lesetexten in der Aufgabe) als auch Sprechen und Schreiben können durch die Arbeit mit Filmen geschult werden. ${ }^{?}$

Diese Auseinandersetzung mit dem Film im DaF-Unterricht kann neben der obligatorischen Arbeit mit kompletten Filmen (d.h. der ca. zweistündigen Spielfilmlänge) insbesondere auch am Kleinformat der Filmszene stattfinden. ${ }^{10}$ Die Vorteile des Kleinformats für die Benutzung des Films als historische Quelle liegen darin, dass so

- die Filmszene sich leicht in eine Unterrichtsstunde/-einheit integrieren lässt;

- die Filmszene sich geschichtsthematisch gut kanalisieren lässt;

- die Filmszene mit der Kombination von Geschichtsthema und Analyse der Filmsprache Kontextwissen und Medienkompetenz fördert;

- landeskundliche und linguistische Fähigkeiten miteinander an einem konkreten, überschaubaren Beispiel miteinander kombiniert werden können;

- sich durch die Filmszenen ein zielgerichteter Lernerfolg realisieren lässt.

Im Folgenden sollen diese Überlegungen anhand dreier ausgewählter Beispiele für Filmszenen als historische Quelle mit der Präsentation von Unterrichtsanwendungen konkretisiert werden.

\footnotetext{
${ }_{9}$ Vgl. hierzu auch Leitzke-Ungerer 2009: 12-21.

${ }^{10}$ Vgl. zum „Kleinformat“ auch Abraham; Kepser 2009, S. 174. Dort wird statt des Wortes Filmszene der Begriff „Filmausschnitt“ benutzt. Selbstverständlich bedarf es aber für ein ganzheitliches Verstehen eines Filmes und seiner Mittel des Schauens eines ganzen Films. Der Filmausschnitt ersetzt keinesfalls die Vorzüge, einen Spielfilm „,von vorne bis hinten“ gesehen und durch anschließende Aufarbeitung verstanden zu haben. Vgl. auch zum Einsatz von Filmen im Deutschunterricht Abraham 2006.
} 


\section{Filmbeispiele}

\subsection{Auswahl und Einordnung der Filmbeispiele}

Die Beispiele decken entweder vom Produktionszeitpunkt oder vom Filminhalt her einen Zeitraum von etwa 30 Jahren ab und lassen sich der jüngeren Zeitgeschichte zuordnen. Dabei beziehen sich die Szenen auf unterschiedliche historisch prägnante oder alltagsweltliche Ereignisse. Zwei der sämtlich in Berlin spielenden Werke können hierbei als Spielfilme eingeordnet werden, darunter eine Literaturverfilmung (Herr Lehmann), der andere wiederum als ein so genanntes Biopic (Christiane F.) mit biographisch-dokumentarischem Gehalt. Der dritte Film ist ein klassischer Dokumentarfilm (Prinzessinnenbad). Die Literaturverfilmung wiederum nimmt Bezug auf zeitgeschichtliche Ereignisse (Mauerfall) und verarbeitet sie auf eine fiktive Weise. ${ }^{11}$

Die Beispiele zur Übersicht: „Christiane F. - Wir Kinder vom Bahnhof Zoo“ (BRD 1980, Regie: Uli Edel) erzählt die Geschichte des dreizehnjährigen Mädchens Christiane F., das im Berlin der frühen 1980er Jahre im sozialen Umfeld ihrer Clique heroinabhängig wird und später in der Prostitution landet. Der Film basiert auf der Lebensgeschichte der Protagonistin. Die Komödie „Herr Lehmann" (BRD 2003, Regie: Leander Haussmann, nach dem gleichnamigen Roman von Sven Lehmann) gibt einen Einblick in das Leben des Barbesitzers Frank Lehmann, gespielt von Christian Ulmen, der in Berlin-Kreuzberg eine Kneipe betreibt. Rahmenhandlung ist der Fall der Mauer 1989. Der Kinoerfolg „Prinzessinnenbad“ (BRD 2007, Dokumentarfilm, Regie: Bettina Blümner) zeigt dokumentarisch den Alltag dreier jugendlicher Berliner Mädchen aus dem Stadtteil Berlin-Kreuzberg.

Neben dem einheitlichen Schauplatz ist allen Filmen gemein, dass sie die Alltagswelt ihrer Hauptfiguren abbilden, deren Milieus sich kreuzen. ${ }^{12}$ Dadurch erhalten die Filmszenen auch einen sozialhistorischen Wert. Die drei Filme repräsentieren in ihrer Thematik dabei soziale und politische Zustände aus drei Perioden der jüngeren deutschen Geschichte.

\subsection{Lernziel und Lerngruppe}

Die jeweiligen Filmszenen bieten sich für folgende Lernziele an: Die Lerner sollen anhand der Kleinformate ihre sprachlichen Kenntnisse in den Bereichen der Grammatik, des Ausdrucks, des Wortgebrauchs und der mündlichen Sprechfertig-

\footnotetext{
11 Vgl. zur Ästhetik von Literaturverfilmungen Bohnenkamp 2005.

12 Bei Christiane F. und Prinzessinnenbad kann man von einem Jugendmilieu sprechen, das sich durch Merkmale wie Musikgeschmack, zwischenmenschliche Beziehungen und „typische“ Probleme Heranwachsender (Schule, Verhältnis zu Drogen und Narkotika, Eltern, Gelderwerb usw.) auszeichnet. Selbst für den Film „Herr Lehmann“ können mit den Verweisen auf die Musikkultur, eine Trink- und alternative Kneipenkultur, die Lebensfragen eines Endzwanzigers sowie Beziehungsprobleme Parallelen gezogen werden.
} 
keit verbessern und vertiefen. Dabei wurden teilweise in die Aufgaben historische Bezüge wie Mauerfall, West-Berliner Drogenmilieu der 1980er Jahre oder zeithistorische Jugendkultur in Berlin eingewoben, um die beiden oben genannten Bereiche zu kombinieren. Es wurde versucht, beide Ebenen ausgewogen zu verbinden; vereinzelt können aber entweder historische oder linguistische Fragstellungen in der einzelnen Aufgabe dominieren. Es wurde außerdem darauf geachtet, die Übungen im Hinblick auf handlungs- und produktionsorientiertes Lernen zu gestalten. Auf medienvertiefende Mittel wie Filmbearbeitung oder spezielles Arbeiten am Computer wurde bei der Konzeption der Übungen wiederum verzichtet.

Die Aufgaben beziehen sich nach dem Europäischen Referenzrahmen für Fremdsprachen auf die Niveaus A2-B2; es wurde bei der Erstellung vornehmlich an erwachsene Lerner gedacht. ${ }^{13}$ Die einzelnen Aufgaben pro Filmausschnitt bauen nicht aufeinander auf und können jeweils selbstständig im Unterricht eingesetzt werden. Dauer, Lernziel und Sozialform der Aufgabe wird im Einzelnen immer angegeben.

\subsection{Aufgaben}

\section{Beispiel 1: Christiane F. - Wir Kinder vom Babnhof Zoo}

Lehrerinformation: Die Szene zeigt die Situation der jugendlichen Drogenabhängigen, die sich auf der Rückseite des Bahnhofs Zoo prostituieren, um so Geld für den nächsten Drogenkauf zu bekommen. Christiane F. bringt ihnen ein paar Butterbrote vorbei und gerät dabei in ein Streitgespräch mit ihrem Freund Detlev. Die Sequenz beginnt bei Minute 01:10:05 und endet bei 01:12:07.

Niveau:

B1

Zeit:

Sozialform:

ca. 45 Minuten

Lerntätigkeiten: Gruppenarbeit (à 2 oder 3 Personen) schriftliches Nacherzählen; Konzentration auf ein Szenenelement ${ }^{14}$ im historischen Kontext; Textverständnis (Grammatik oder Vokabular); Erstellen eines Lückentextes für andere Teilgruppen.

\footnotetext{
13 Allerdings findet das Niveau A2 nur einmalig Verwendung, was mit der niveauadäquaten Ausrichtung auf Spracherwerb zusammenhängt und leider weniger Spielraum zur Verbindung mit komplexerem, in diesem Falle historischem Wissen erlaubt.

${ }^{14}$ Der Begriff „Szenenelement“ bezeichnet alles, was in einer Szene repräsentiert wird, also vom materiellen Gegenstand wie einem Schlüssel bis zum Dialog oder Klängen. Die Lehrkraft sollte vorab den Begriff verständlich machen, damit die Lerner wissen, was in der Aufgabe damit gemeint ist.
} 
Aufgabe 1:

Setzen Sie sich mit 2 oder 3 Personen aus Ihrem Kurs zusammen. Schauen Sie sich die Szene zwei- bis dreimal an. Achten Sie in Ihrer Gruppe auf „Ihr“" Szenenelement:

Teilgruppe 1 achtet auf die Kleidung und das Aussehen der Figuren

Teilgruppe 2 achtet auf die Passanten

Teilgruppe 3 achtet auf den Verkehr und die Umgebung

Teilgruppe 4 achtet auf die Dialoge der Figuren

Teilgruppe 5 achtet auf das Thema der Szene

Erzählen Sie die Szene in Ihrer Teilgruppe nach. Orientieren Sie sich an den folgenden Fragen: In welcher Zeit spielt die Szene? Wie finden Sie das Aussehen der Figuren? Wo spielt die Szene? Was sieht man überhaupt? Was machen die Figuren? Was sagen die Figuren? Was machen die Passanten?

Aufgabe 2:

Erzählen Sie die Szene nochmals nach und heben Sie in Ihrer Nacherzählung Ihr Szenenelement (Kleidung oder Umgebung oder Dialoge oder Thema) diesmal besonders hervor, d.h. es soll besonders oft in Ihrem Text vorkommen. Fügen Sie dann Lücken in ihren Text ein, in denen entweder nach der grammatischen Form eines Faktors (z.B. „die dunkl__ (= dunklen) Haare des Jungen") oder nach dem passenden Ausdruck für ein Wort (z.B. ,der Junge trägt eine _ jacke (= Jeansjacke)") gesucht wird. Tauschen Sie danach ihren Text mit einer anderen Teilgruppe und füllen Sie die Lücken aus.

\section{Beispiel 2: Herr Lehmann}

Lehrerinformation: Die Szene spielt in einer Kneipe in Kreuzberg nahe der UBahnstation „Kottbusser Tor“. Herr Lehmann betrinkt dort seinen Liebeskummer. Die Frau mit den dunklen Haaren neben ihm informiert die gesamte Kundschaft über das Ereignis der Maueröffnung am 9. November 1989. Die Szene erhält ihre Komik durch die verhaltene, fast desinteressierte Reaktion vieler Gäste. Die Szene beginnt bei Minute 01:36:35 und endet bei 01:37:50.

Niveau:

Zeit:

Sozialform:

Lerntätigkeiten:

\section{B1-B2}

30-45 Minuten

Gruppen- und Einzelarbeit

Finden eines gemeinsamen Themas in einer Gruppe, Erstellen von Einzeldialogen zum Thema, freies Sprechen (Synchronisation von Schauspielern); schriftliches Begründen, Aufnahme und Verarbeitung von historischem 
Grundwissen; Interpretation von Figurenverhalten und Szenenästhetik.

Aufgabe 1:

Schauen Sie sich die Szene mehrmals ohne Ton in einer Gruppe von vier Personen an. Jeder der Gruppenteilnehmer übernimmt eine Rolle der Szene: 1. Herr Lehmann; 2. Herr Lehmanns Freund Silvio; 3. der weibliche Gast; 4. der Barkeeper. Überlegen Sie sich in Ihrer Gruppe ein Thema für die Szene (Beispiel: „Liveschaltung aus Deutschland: Das Volk jubelt. Das neue Superauto ist endlich da!"). Überlegen Sie sich dann individuell Sätze, die ihre Rolle in der Szene sprechen soll. Achten Sie dabei auf die Länge ihrer Sätze, so dass Sie zu den Mundbewegungen der Figuren passen. Lassen Sie anschließend die Szene ohne Ton laufen und synchronisieren Sie die Szene mit ihren Dialogen. Wiederholen sie dies mehrmals.

Aufgabe 2:

Schauen Sie sich die Szene mehrmals an und beantworten sie schriftlich die folgenden Aufgaben. Begründen Sie Ihre Antworten. Welches Ereignis steht im Mittelpunkt der Szene? Was sieht man für Bilder im Fernsehen? Welcher Zeit und welchem Ereignis würden Sie diese Fernsehbilder zuordnen? Warum?

Aufgabe 3:

Schauen Sie sich die Szene an und achten Sie auf das Verhalten der Figuren und auf die Szenen, die im Fernseher gezeigt werden. Beantworten Sie folgende Fragen schriftlich: Wie verhalten sich die Figuren in der Bar, wie die Menschen in den Fernsehbildern? Die Szene wirkt auf den Zuschauer komisch. Warum?

\section{Beispiel 3: Prinzessinnenbad}

Lehrerinformation: Der Dokumentarfilm „Prinzessinnenbad“ zeigt ein Jahr im Leben dreier jugendlicher Mädchen in Berlin-Kreuzberg. Der Filmtitel verweist bereits auf die dazugehörige U-Bahnstation in Berlin-Kreuzberg, die Prinzenstraße auf der Linie U 1. Der Ausschnitt zeigt eine mit Musik unterlegte Ansicht der Gegend nahe der U-Bahnstation „Kottbusser Tor“. Die Szene beginnt bei 00:06:11 und endet bei 00:06:45.

Aufgabe 1.1:

Niveau: A2

Zeit: 30-40 Minuten

Sozialform: Einzelarbeit

Lerntätigkeiten: Leseverstehen von Aussagen und Zuordnen richtiger/falscher Antworten 
Die folgende Szene besteht hauptsächlich aus Einstellungen bzw. 6 Bildern. Sind die folgenden Sätze richtig oder falsch? Schauen Sie sich die Szene mehrmals an.

Bild 1: Man sieht nur ein Taxi in dem Bild.

$\mathrm{r} / \mathrm{f}$

Bild 2: Ein weißer Lastwagen ist vor einem roten Torbogen zu sehen. $\quad \mathrm{r} / \mathrm{f}$

Bild 3: Es sind keine Passanten auf der Straße zu sehen. r/f

Bild 4: Man sieht eine gelbe U-Bahn fahren. $\mathrm{r} / \mathrm{f}$

Bild 5: Eine Person auf einem Motorrad fährt im Bildvordergrund her. $\mathrm{r} / \mathrm{f}$

Bild 6: Der Bus hat die Nummer M 29 und fährt in Richtung Roseneck. r/f

Aufgabe 1.2:

Schreiben Sie einen kurzen Erzähler-Kommentar („voice over") zu den Einstellungen, indem Sie die Umgebung um das Kottbusser Tor präsentieren. Tragen Sie Ihren Kommentar zu den laufenden Bildern laut vor.

Aufgabe 3:

Niveau:

B2

Zeit:

ca. 30 Minuten

Sozialform:

Einzelarbeit

Lernziel:

Schauen Sie sich die Szene ein- bis zweimal an. Lesen Sie anschließend folgende Zeilen, die aus einem Artikel der Wochenzeitung „Die Zeit“ vom 03.07.2003 stammen:

„Berlin-Kreuzberg, Kottbusser Tor: Deutschlands älteste Sanierungs-Story geht nach fast vier Jahrzehnten zu Ende. Die Hoffnung, mit Städtebau Sozialpolitik betreiben zu können, ist gescheitert. Spaziergang durch ein aufgegebenes Biotop.“

Beantworten Sie schriftlich: Was sind dem Artikel nach die Besonderheiten des Bezirks rund um das Kottbusser Tor? Vergleichen Sie die Aussagen mit den Filmbildern. Diskutieren Sie, inwiefern die Bilder diesen Aussagen entsprechen oder nicht.

\subsection{Unterrichtserfahrungen mit einzelnen Beispielen}

Zwei der genannten Beispielfilme ${ }^{15}$ kamen in einer französischen Universität in den Jahrgängen der Licence 2 (vgl. mit dem Bachelor 2. Studienjahr) in den Kursen

\footnotetext{
${ }^{15}$ Das dritte Beispiel wurde eigens für diesen Text entworfen und konnte daher noch nicht im Unterricht erprobt werden.
} 
„Expression et compréhension écrites“ und „Pratique orale“ zum Einsatz. Das Niveau der Lerner kann in beiden Fällen auf B1 angesiedelt werden.

In den „Expression écrite“-Kursen wurden die Lerner in fünf Teilgruppen z.B. gebeten, sich die „Christiane F.“-Filmszene zweimal anzuschauen und sich während des Schauens Notizen zur Szene (Kleidung, Umwelt, mündlicher Sprachgebrauch, Thema des Dialogs) zu machen. Texte zum Merkmal aus der jeweiligen Zeit waren dazu in den vorherigen Unterrichtsstunden mit den Teilnehmern bearbeitet worden, so dass die Lerner auf ein passendes Vokabular und eine historische Einordnung zurückgreifen konnten. Im Anschluss daran wurden die Lerner aufgefordert, mittels ihrer Notizen die Filmszene in ihrem Verlauf schriftlich wiederzugeben, wobei jeder Lerner sich auf eines der Merkmale (s. Aufgabe) konzentrieren sollte. Dazu wurde parallel die Aufgabe gestellt, den Handlungsverlauf in einen historischen Rahmen einzubetten und dabei jeweils das Merkmal gemäß der Zeit zu thematisieren.

Im „Pratique orale“-Kurs erhielten die Teilnehmer die Szene aus dem Film „Herr Lehmann“ mit der Aufgabenstellung, die Szene ohne Ton dreimal gut anzusehen. Hinterher wurden sie dann in Partnerarbeit gebeten, zu dieser Szene einen Fernsehbericht zu halten, d.h. sie sollten in die Rolle eines Reporters schlüpfen, der die zweiminütige Szene für ein fiktives Fernsehpublikum kommentiert. Das Fernsehpublikum stellten dann die anderen Teilnehmer dar, während die beiden Lerner abwechselnd ihren Kommentar zu den Bildern vorlasen. Damit dieser nicht zu stockend abgelesen klingt, bekamen sie vorher zusätzliche Zeit für eine Leseprobe. Die Lerner wurden dabei von einem Abspielgerät (z.B. I-pod) aufgenommen und konnten sich hinterher ihre Leistung anhören, wobei die Lehrkraft die Stellen notierte, in denen die Satzmelodie oder die Wortbetonung verändert werden musste. Auf diese Weise konnten sich die Lerner in ihrem Sprechfluss verbessern. Von Vorteil in dieser Stunde war, dass die Lerngruppe nur aus vier Personen bestand. Bei einer größeren Gruppe könnte man in diesem Fall die Aufgabe zur Abgabe eines themengebundenen Berichts vergeben, d.h. die Szene soll in einem neuen Kontext wie z.B. einer „Fußballspielberichterstattung“ oder eines „politischen Staatsbesuchs" nacherzählt werden.

In beiden Gruppen war die Resonanz auf die Übungen weitestgehend positiv, allerdings war in der schriftlichen Aufgabe zur Filmszene „Christiane F.“ weitaus mehr Betreuung sowohl im Verstehen als auch während der Aufgabenfertigstellung nötig. Dies gilt es bei der Durchführung zu beachten, um so vor allem schwächeren Kursteilnehmern stärker zu helfen und ggf. die Gruppeneinteilung vom Niveau her ausgewogener vorzunehmen. 


\section{Zusammenfassung}

Bei der Arbeit mit Filmszenen als historischen Quellen stellt sich die Frage, mit welchen didaktischen Mitteln man diese in den DaF-Unterricht integriert: Oft wird der Film en bloc gezeigt und dann die Variante des Vortrags gewählt, und man erklärt am Film die jeweiligen Zeitumstände in einer Plenardiskussion oder im Frontalunterricht. Eher selten folgen aus der Beschäftigung damit sprachkompetenzorientierte Übungen, d.h. Aufgaben, die versuchen, das historische Wissen so niveau-adäquat zu vermitteln, dass der Lerner parallel dazu seine Sprachfähigkeit selbstständig trainieren kann. Das Ziel hierbei ist, dem Lerner die in einer Filmszene enthaltenen geschichtlichen Informationen als Herausforderungen zum Erwerb (höherer) Sprachkompetenz anzubieten. Die drei oben erwähnten Beispiele, die teilweise auch im Unterricht ihre Anwendung fanden, versuchen dies zu belegen und laden Kolleginnen und Kollegen ein, sie ebenfalls auszuprobieren.

Es hat einige Zeit gebraucht, bis der Film als Arbeitsmittel im Geschichts- und DaF-Unterricht vollständig anerkannt wurde. Dass der Film als solcher sich, zumindest als historische Quelle für den DaF-Unterricht schließlich hat durchsetzen können, verdankt er sicherlich auch der strukturellen Offenheit des Fachs, Material aus verschiedenen lebensnahen Kontexten aufzunehmen und zu didaktisieren.

\section{Literatur}

Abraham, Ulf; Kepser, Matthis ('2009): Literaturdidaktik Deutsch. Eine Einführung. Berlin: Erich Schmidt.

Abraham, Ulf (2006): Mehr als nur „Theater mit Videos“. Theatralität in einem medienintegrativen Deutschunterricht und szenische Verfahren im Umgang mit Film und Fernsehen, in: Frederking, Volker (Hrsg.): Jabrbuch Medien im Deutschunterricht 2005. Filmdidaktik und Filmästhetik. München: kopaed, 130-144.

Behrens, Ulrich (2009): Geschichte im Film - Film der Geschichte. Norderstedt: Books on Demand.

Bohnenkamp, Anne (2005): Vorwort. In: Bohnenkamp, Anne/Lang, Tilman (Hrsg): Interpretationen Literaturverfilmungen. Stuttgart: Reclam, 9-33.

Gehrke, Ulrich (2011): Veit Harlan und der „Kolberg“-Film. Filmregie zwischen Geschichte, NS-Propaganda und Vergangenheitsbewältigung. Hamburg: Hamburg

Marmorweg 14: U. Gehrke.

Hickethier, Knut (32007): Film- und Fernsehanalyse. Stuttgart und Weimar: Metzler.

Koller, Wolfgang: Die Erinnerungskultur der Revolutions- und Napoleonischen Kriege im europäischen Kino, 1914 bis 1945 (in Vorbereitung). 
Kotulla, Theodor (1964): Der Film. Manifeste, Gespräche, Dokumente Bd.2. 1945 bis beute. München: Piper.

Leitzke-Ungerer, Eva (Hrsg.) (2009): Film im Fremdsprachenunterricht. Literarische Stoffe, interkulturelle Ziele, mediale Wirkung. Stuttgart: Ibidem.

Schanze, Helmut (Hrsg.) (1996): Fernsehgeschichte der Literatur. VoraussetzungenFallstudien - Kanon. München: Fink.

Wedel, Michael (2011): Filmgeschichte als Krisengeschichte. Schnitte und Spuren durch den deutschen Film. Bielefeld: Transkript.

\section{Filme}

Christiane F. - Wir Kinder vom Bahnhof Zoo. BRD 1981, Regie: Ulrich Edel.

Herr Lehmann. BRD 2003. Regie: Leander Haussmann.

Prinzesinnenbad. BRD 2007. Regie: Bettina Blümner. 



\title{
Historische Audio- und Videodokumente im DaF-/Phonetikunterricht - Vorschläge und Erfahrungen aus der Unterrichtspraxis
}

\author{
Beatrice Wiegand
}

\section{Einleitung}

Historische Tondokumente wirken auf heutige Hörer manchmal übertrieben gesprochen und seltsam ,aus der Zeit gefallen“. Während sie im landeskundlichen DaF-Unterricht häufig eine Abwechslung und Bereicherung darstellen, erscheinen sie für den Sprachunterricht aus diesem Grund hingegen eher ungeeignet. Dieser Beitrag will dennoch für den Einsatz historischer und zeithistorischer Audio- und Videodokumente im Sprach- und Phonetikunterricht werben, beziehungsweise solcher Dokumente, deren Sprecher sich wie der Sänger Max Raabe sehr stark an historische Vorbilder angelehnt haben oder sich in einem Kontext jahrzehntelanger Traditionen bewegen, die sie aufgreifen oder bewusst abzuwandeln versuchen, wie der Bundespräsident in seiner Weihnachtsansprache.

Solche Hördokumente - im Folgenden zusammengefasst „historische“ Dokumente genannt - können nach Erfahrung der Verfasserin in besonderer Weise für phonetische und klangliche Aspekte der Sprache sensibilisieren. Dazu gehören Aussprachephänomene wie Akzentuierung, Rhythmisierung und Melodisierung im Deutschen, aber auch Aussprachevarianten und die sich in ihnen realisierenden Sprechabsichten (Ironie, Feierlichkeit, Spannungserzeugung usw.). Gerade dort, wo die Aussprache stilistische Auffälligkeiten aufweist, möglicherweise von heutigen Hörgewohnheiten abweicht und übertrieben erscheint, werden Gestal- 
tungsmittel und Realisierungsformen oft besonders deutlich. Das erleichtert erstens die Wahrnehmung phonetischer Aspekte, zweitens kann dies in einer anschließenden Phase sprachlicher Produktion einen spielerischen Kontext und Freiraum bieten.

Der Einsatz historischer Audio- und Videodokumente erscheint außerdem dadurch gerechtfertigt, dass es sich um authentische Materialien handelt, denen die Lerner auch außerhalb des Sprachunterrichts begegnen können, und anhand derer sich Hörerfahrungen erweitern lassen. Auch in Sprachprüfungen, z.B. den Zertifikatsprüfungen des Goethe-Instituts oder den auf diese Prüfungen vorbereitenden Unterrichtsmaterialien begegnen Deutschlerner verschiedenen Varianten der deutschen Sprache und Aussprache.

Im Folgenden sollen Unterrichtseinheiten vorgestellt werden, die auf historischen und zeithistorischen Audio- und Videodokumenten basieren und anhand derer verschiedene sprachliche Fertigkeiten, vor allem aber phonetische Aspekte des Deutschen thematisiert und geübt werden. Die einzelnen Beispiele sind als Anregungen für den Sprachunterricht gedacht.

\section{Unterrichtsentwurf 1: „Mein kleiner grüner Kaktus“}

Interpretation: Max Raabe \& Palast Orchester

Musik: Albrecht Marcuse; Bert Reisfeld

Text: Hans Herda, 1934

Blumen im Garten, so zwanzig Arten, von Rosen, Tulpen und Narzissen, leisten sich heute die feinen Leute, das will ich alles gar nicht wissen.

Mein kleiner grüner Kaktus steht draußen am Balkon, hollari, hollari, hollaro.

Was brauch' ich rote Rosen, was brauch' ich roten Mohn, hollari, hollari, hollaro.

Und wenn ein Bösewicht was Ungezog'nes spricht, dann hol' ich meinen Kaktus und der sticht, sticht, sticht. Mein kleiner grüner Kaktus 
steht draußen am Balkon,

hollari, hollari, hollaro.

Heute um viere

klopft's an die Türe.

Nanu, Besuch so früh am Tage?

Es war Herr Krause

vom Nachbarhause,

der sagt: Verzeih'n Sie, wenn ich frage...

Sie ham' doch einen Kaktus

da draußen am Balkon,

hollari, hollari, hollaro.

Der fiel soeben runter,

was halten Sie davon?

Hollari, hollari, hollaro.

Er fiel mir auf's Gesicht, ob's glauben oder nicht, jetzt weiß ich, dass Ihr kleiner grüner Kaktus sticht.

Bewahr'n Sie Ihren Kaktus

gefälligst anderswo,

hollari, hollari, hollaro.
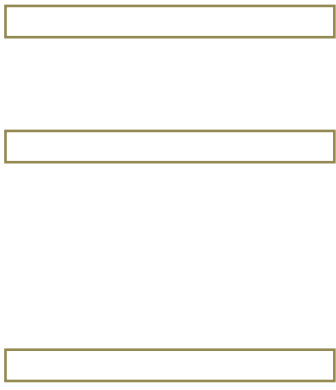

\section{Aufgabenstellungen}

1. Hören Sie das Lied „Mein kleiner grüner Kaktus“ in der Interpretation von Max Raabe! Wie finden Sie das Lied?

2. Hören und sehen Sie nun den Auftritt noch einmal! Charakterisieren Sie die Sprechweise, indem Sie die folgenden Adjektive an den passenden Stellen in den Kästen neben dem Liedtext oben ergänzen!

vergnügt/gut gelaunt verärgert
Spannung erzeugend
misstramisch

3. Mit der Stimme kann man Emotionen, Ironie, Spannung usw. erzeugen. Womit noch?

4. Wo in dem Lied und wie erzeugt Raabe Ironie? 
5. Sprechen Sie die folgenden Textstellen jetzt mit unterschiedlicher Sprechweise! Sprechen Sie so, wie auf dem Los gefordert! Die anderen raten/erkennen, wie Sie gesprochen haben!

\begin{tabular}{|c|c|c|c|c|}
\hline $\begin{array}{c}\text { vergnügt/ } \\
\text { gut gelaunt }\end{array}$ & verärgert & misstrauisch & Aufgeregt & $\begin{array}{c}\text { erstaunt/ } \\
\text { verwundert }\end{array}$ \\
\hline $\begin{array}{c}\text { gelangweilt/ } \\
\text { gleichgültig }\end{array}$ & Traurig & schüchtern & ganznormal & wie Max Raabe \\
\hline
\end{tabular}

\title{
Nach: Endt; Hirschfeld (1995: 54).
}

\author{
Was brauch' ich rote Rosen, \\ was brauch' ich roten Mohn, \\ Dann hol ich meinen Kaktus \\ und der sticht, sticht, sticht. \\ Nanu, Besuch so früh am Tage? \\ Verzeih'n Sie, wenn ich frage... \\ Sie ham' doch einen Kaktus \\ da draußen am Balkon... \\ ... der fiel soeben runter, \\ was halten Sie davon? \\ Bewahr'n Sie ihren Kaktus \\ gefälligst anderswo auf!
}

\section{Ziele und Erfahrungen mit dem Unterrichtsentwurf}

Bei der im Unterricht verwendeten Videoaufnahme handelt es sich nicht um eine historische Aufnahme aus der Entstehungszeit des Liedes, also den 1930er Jahren des 20. Jahrhunderts, sondern um eine Interpretation Max Raabes aus dem Jahr 2006, im Stil der Entstehungszeit, aber auch im persönlichen Stil des Interpreten. ${ }^{1}$

\footnotetext{
${ }^{1}$ Für den vorliegenden Unterrichtsentwurf wurde der Interpretation Raabes der Vorzug vor einer historischen Aufnahme z.B. der Comedian Harmonists gegeben. Zum einen erlaubt dies den Einsatz eines Videos und damit die Berücksichtigung von Gestaltungsmitteln wie Mimik und Inszenierung (Kleidung, Orchester etc.). Zum anderen werden bei Raabe - dessen Interpretation sich auf historische Vorbilder bezieht, jedoch auch übertreibt und ironisiert - Aussprachephänomene besonders deutlich.
} 
Ziel der anhand des Videomaterials entwickelten Unterrichtseinheit, die sich an Lerner ab Niveau B1 richtet, ist eine Sensibilisierung für emotional und situativ bedingte Sprechweisen ${ }^{2}$ und Aussprachevarianten im Deutschen, hier eine bestimmte Emotionalisierung und Ironisierung. In Aufgabe 2 sollen die Lerner diese Modi des Sprechens erkennen. Die Aufgaben 3 und 4 tragen dem Sachverhalt Rechnung, dass auch andere Gestaltungsmittel zum Ausdruck von Emotionen, Ironie oder Spannung beitragen, was in Raabes sparsam, aber wirkungsvoll eingesetzter Mimik im Video deutlich wird. Aufgabe 5 stellt eine produktive Ausspracheübung spielerischer Form dar und basiert auf der Übungsform „Lies den Text so!“ des Phonetikmaterials „Die Rhythmuslokomotive“ (Endt; Hirschfeld 1995: 54). Anhand der ihnen bereits bekannten Verse aus dem Lied können die Lerner hier selbst emotionale Varianten in der Fremdsprache Deutsch produzieren und ausprobieren. Zur Unterstützung phonetischer Mittel (z.B. Sprechtempo, Lautstärke, Melodieverläufe und andere Parameter der Prosodie) ${ }^{3}$ können die Lerner auch Mimik und Gestik einsetzen.

Im Sprachunterricht mit französischen Studierenden der Germanistik ließ sich zunächst feststellen, dass die Humorlage des Liedes und seiner Vortragsweise durchaus der relativ jungen Zielgruppe entsprach. Als lustig wurden der Kontrast zwischen ernstem festlichem Vortrag (Orchester, Kleidung, Aussprache und Vortragsweise) und dem „lustigen, albernen Text" (Zitate der Studierenden), aber auch die „energische, flotte Musik“ sowie die Tendenz zu Deutlichkeit und Übertreibung empfunden. Während die Körperhaltung als reglos beschrieben wurde, imitierten die Lerner schnell Blick und Mimik Max Raabes. Im Bereich der Aussprache fielen die stellenweise hohe Deutlichkeit sowie die Realisierung der RLaute auf. Beides lässt sich auf eine erhöhte Sprechspannung und Artikulationspräzision ${ }^{4}$ einiger Passagen des Kunstgesangs zurückführen. Auch diese phonostilistische Aussprachevariante konnten die Deutschlerner im Verlauf der anschließenden produktiven spielerischen Übung imitieren, wenn sie gemäß ihrem Los „so wie Max Raabe“ sprechen sollten.

2 Zum Begriff „Sprechweise“ vgl. Reinke 2008: 35ff.

${ }^{3}$ Einen Überblick über Begrifflichkeiten, Realisierungsmittel und Funktionen prosodischer Aspekte des Deutschen bieten Hirschfeld; Neuber 2010: 10-16.

${ }^{4}$ Zur Kategorie der Artikulationspräzision und phonostilistischen Varianten der Aussprache vgl. das Kapitel „Phonostilistische Differenzierungen der Standardaussprache“ in: Krech; Stock; Hirschfeld; Anders 2009: 98ff. 


\section{Unterrichtsentwurf 2:}

\section{„Eine Rede halten - die Weihnachtsansprache 2010“}

\section{Aufgabenstellungen}

1. Sie sehen die Weihnachtsansprache des Bundespräsidenten Christian Wulff aus dem Jahr 2010. Was sind zentrale Themen seiner Rede? Was sind zentrale Botschaften?

2. Sehen Sie das Video nun noch einmal und notieren Sie Ihre Beobachtungen zu den unten genannten Aspekten! Präsentieren Sie anschließend die Beobachtungen Ihrer Gruppe!

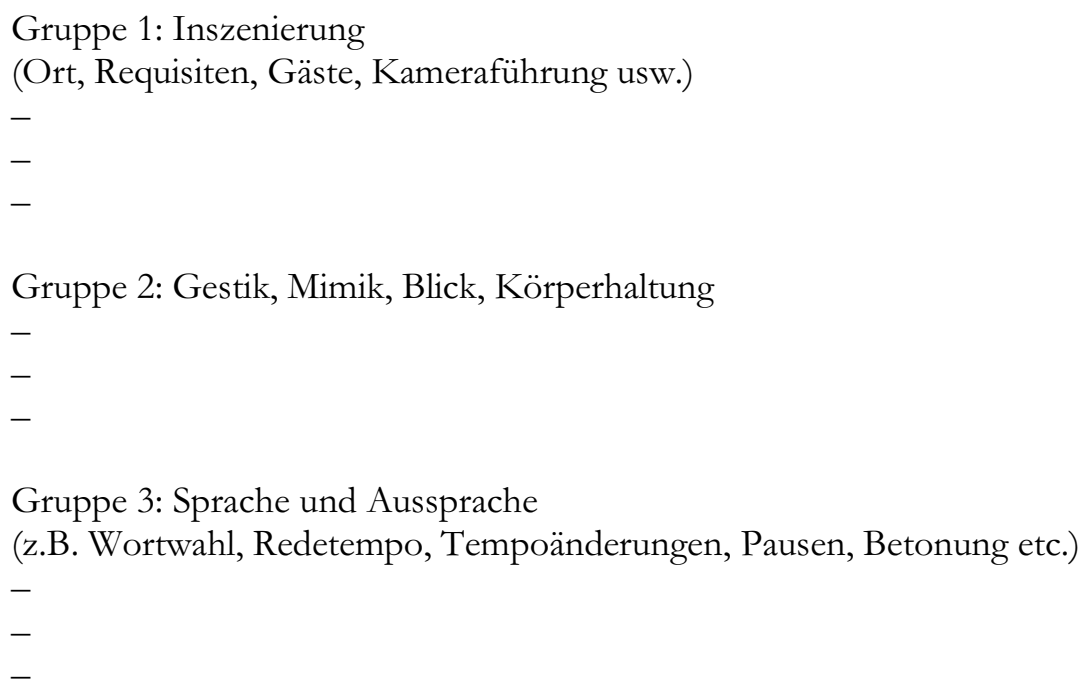

3. Wie wirkt die Rede des Bundespräsidenten auf Sie?

4. Halten Sie nun eine eigene kleine Ansprache! Die Situation ist die folgende: Sie haben ein dreimonatiges Praktikum in Deutschland absolviert, das nun zu Ende geht. Aus diesem Anlass geben Sie für Ihre zehn KollegInnen einen Ausstand. Sie haben ein kollegiales Verhältnis, sind aber keine „Kumpel“. Sie möchten sich positiv äußern und versprechen sich auch in Zukunft etwas von Ihren Praktikumsgebern, möchten sich aber auch nicht einschmeicheln. Sie haben einen Kuchen gebacken und bevor Sie diesen gemeinsam essen, richten Sie ein paar Worte an die Runde. Bereiten Sie einige Sätze zu den folgenden Inhaltspunkten vor! 
- Resümee des Praktikums

- Dank für die Zusammenarbeit

- Hoffen auf weiteren Kontakt

Halten Sie anschließend Ihre kleine Rede und benutzen Sie dabei eigene Mittel der Gestik, Mimik, Sprache und Aussprache!

\section{Ziele und Erfahrungen mit dem Unterrichtsentwurf}

Die Idee, eine Weihnachts- oder Neujahrsansprache zu analysieren, ist nicht neu. Eine interessante kontrastierende Perspektive eröffnet beispielsweise die Sendung „Karambolage“ des Fernsehsenders Arte, indem sie eine französische und zwei deutsche Ansprachen gegenüberstellt (vgl. Clairon; Doutriaux 2005). Was nun die Weihnachtsansprache des Bundespräsidenten Christian Wulff aus dem Jahr 2010 für den Fremdsprachenunterricht Deutsch meines Erachtens interessant macht, betrifft einige Merkmale der Gestaltung seiner Rede, die auf eine direkte Ansprache des Adressaten zielen und in ihrer beabsichtigten Wirkung vielleicht am besten damit beschrieben werden können, dass sie „Verbindlichkeit“ herstellen sollen.

In der vorgeschlagenen Didaktisierung dient das erste Hören und Ansehen des Dokuments der Erarbeitung seiner inhaltlichen Seite. Die Aufgabenstellung, zentrale Themen und Inhalte der Rede herauszuhören, ist als Training des globalen Hörverstehens angelegt. Dieser inhaltlichen Auseinandersetzung folgt eine Analyse verschiedener Aspekte der Präsentation. Vorgeschlagen wird dabei eine Aufteilung der Beobachtungsaspekte auf drei Gruppen: Gruppe 1 konzentriert sich auf Aspekte der Inszenierung (Ort, Requisiten, Gäste, Kameraführung usw.), Gruppe 2 beobachtet Gestik, Mimik, Blick und Körperhaltung des Redners und Gruppe 3 macht Notizen zu Sprache und Aussprache (z.B. Wortwahl, rhetorische Mittel, Redetempo, Tempoänderungen, Pausen, Betonung usw.). Im Anschluss an ein zweites Ansehen des Videos erhalten die Studierenden einige Minuten Zeit, um innerhalb ihrer jeweiligen Gruppe Beobachtungsergebnisse auszutauschen und zu diskutieren. Danach präsentieren die Gruppen ihre Beobachtungen im Plenum. Die Frage 3 des Unterrichtsentwurfs, „Wie wirkt die Rede des Bundespräsidenten auf Sie?", zielt unter anderem auch darauf, die zunächst differenzierten Analyseaspekte und Blickpunkte in der Interpretation ihrer Wirkung wieder zusammenzuführen.

In der Unterrichtspraxis war interessant zu sehen, wie die Interpretationen und Bewertungen der Rede durch verschiedene Lernergruppen auseinander ging. Während Gestik und Mimik in einem ersten Unterricht beispielsweise mit „steht aufrecht, gerade; lächelt, blickt freundlich" charakterisiert wurden, beschrieben Studierende eines anderen Kurses dasselbe Verhalten als „steht steif, bewegt nur die Hände; lächelt nur einmal“". Bei der Frage nach der Wirkung der Rede führte das zu Wertungen wie „freundlich, nah, nicht distanziert“ innerhalb der ersten 
Lernergruppe, während die Gruppe aus der anderen Unterrichtseinheit „feierlich, offiziell, ernst, optimistisch, idealisierend, klischeehaft" urteilte.

In beiden Unterrichtsstunden führte ich den Begriff und die Kategorie der „Verbindlichkeit“ der Ansprache ein, auf welche meines Erachtens sowohl Inszenierung als auch Gestik und Mimik sowie Sprache und Aussprache zielen. Die Studierenden selbst erwähnten in diesem Kontext den Blick als Kontaktmittel, aber auch den phonetischen Aspekt einer starken Akzentuierung „wichtiger Wörter", zentraler Begriffe der Rede und der Anreden von Personengruppen.

Bei der Konzeption der produktiven Aufgabe, zu zweit eine eigene kleine Ansprache zu entwerfen und zu präsentieren, ging es vor allem darum, eine adäquate realitätsnahe Situation zu schaffen und diese möglichst konkret vor Augen zu führen. Die Lerner wurden schriftlich und mündlich dazu angehalten, bei der Gestaltung ihrer Ansprache nach „eigene(n) Mittel(n) der Gestik, Mimik, Sprache und Aussprache" zu suchen. Die präsentierten Ergebnisse wiesen, auch im Einsatz von Blick und Gestik, deutliche Unterschiede und persönliche Züge auf. Eine Aufzeichnung mit Videokamera kann hinsichtlich der Präsentation sicher zu noch mehr Ernsthaftigkeit führen und in der Auswertungsphase noch fundiertere Reflexion erlauben.

\section{Unterrichtsentwurf 3:}

\section{„Die Prinzessin auf der Erbse - ein Märchen vortragen“}

Text:

Vortrag:

Hans Christian Andersen

Alexander Moissi, 1928

\section{Aufgabenstellungen}

1. Welche Merkmale charakterisieren in der Regel den Vortrag eines Märchens? Kreuzen Sie an, was Ihrer Meinung nach zutrifft!

\author{
Man spricht meist \\ Pausen macht man \\ Die rhythmischen Gruppen sind \\ (vgl. Stock 1996: 71)
}

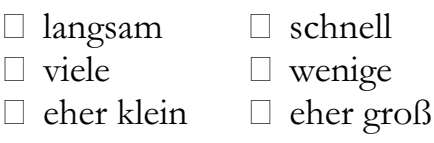

eher klein $\square$ eher groß

2.a Hören Sie nun den Vortrag des Schauspielers Alexander Moissi aus dem Jahr 1928! Wie finden Sie seine Vortragsweise? Was charakterisiert sie?

2.b Welche Merkmale sind stark der Zeit (den 1920er Jahren) verhaftet? Was würde Sie heute anders machen? 
3. Auch wenn Ihr eigener Vortrag heute ganz anders ist, Akzente, Melodieverläufe und sinnfällige Pausen werden in dem historischen Vortrag doch sehr deutlich. Hören Sie den Text jetzt noch einmal! Markieren Sie - mit Bleistift - Pausen und wichtige Satzakzente!

Es war einmal ein Prinz, // der wollte eine Prinzessin heiraten. // $\underline{\text { Aber } / \text { es }}$ sollte eine wirkliche Prinzessin sein. Er reiste nun in der ganzen Welt umher, um eine solche zu finden, aber immer kam etwas dazwischen. Prinzessinnen waren ja genug da, aber ob es eine wirkliche Prinzessin war, dahinter konnte er durchaus nie kommen. Immer war irgend etwas, das nicht recht stimmte. So kam er denn wieder nach Hause und war ganz traurig, denn er hätte doch so gern eine wirkliche Prinzessin gehabt. [...]

4. Überprüfen Sie, ob Sie selbst dieselben Akzente und Pausen setzen würden! Ändern Sie eventuell Ihre Markierungen und bereiten Sie einen eigenen Vortrag vor! Machen Sie eine Aufnahme!

\section{Ziele und Erfahrungen mit dem Unterrichtsentwurf}

Den Vortrag eines Märchens kennzeichnen spezifische prosodische Merkmale. Insbesondere in Bezug auf Sprechtempo und Pausen können Lerner diese Textsorte sicher schnell beschreiben (vgl. Aufg. 1). Märchen kennzeichnet gemäß Stock (1996: 71) darüber hinaus, dass sie aus relativ kleinen rhythmischen Gruppen bestehen. Das prädestiniert sie für die Arbeit an prosodischen Aspekten wie Akzentuierung, Rhythmus, Pausierung und Melodisierung. Der Unterrichtsvorschlag setzt sich aus zwei angewandten Übungen ${ }^{5}$, einer Hörübung und einer textgebundenen Sprech- und Ausspracheübung zusammen. Da das zu hörende Tondokument, eine Aufnahme des Schauspielers Alexander Moissi, ganz allgemein von unseren heutigen Hörgewohnheiten abweichen dürfte und beim Hören erst einmal sicher Heiterkeit verursacht, wird den Lernern zunächst die Frage nach phonetischen und sprechkünstlerischen Besonderheiten dieses Vortrags gestellt. Im CD-Cover werden Vortragsweise und Wirkung wie folgt beschrieben:

„Auch dem Laien werden im Vergleich mit unseren heutigen Hörgewohnheiten die Empathie, die „Übertreibung“, das Theatralische, Pathetische nicht verborgen bleiben. Es entsprach den sprechstilistischen Auffassungen dieser Zeit, dass mehr „deklamiert“ denn „rezitiert" wurde. Auch der Grad der Artikulationspräzision scheint heute überhöht. Besonderheiten wie das sogenannte rollende R sind dem damaligen Zeitgeschmack zuzuschreiben.“ (Müller: 2004)

${ }^{5}$ Die Klassifizierung und Charakterisierung der Übungen folgt in diesem Artikel der Typologie in Dieling; Hirschfeld 2000: 47ff. 
Der übertreibende Gestus in Moissis Vortrag führt dazu, dass prosodische Merkmale und die Mittel ihrer Realisierung besonders deutlich hervortreten, was die Aufnahme meines Erachtens für den Einsatz im Phonetikunterricht interessant macht. Die starke Empathie und Dramatik seiner Interpretation des Märchens realisiert er nicht nur mittels verstellter Stimme in Passagen direkter Figurensprache, sondern auch mittels starker Akzentuierung. Auch die Mittel der Akzentuierung im Deutschen (erhöhte Lautstärke und Sprechspannung, herausgehobene Tonhöhe, verlangsamtes Sprechtempo, vgl. Hirschfeld; Neuber 2010: 12) treten deutlich hervor. Wenn auch den Lernern in jedem Fall deutlich werden muss, dass eine zeitgemäße Interpretation des Märchens heute anders klingt, prädestiniert diese Deutlichkeit prosodischer Aspekte und ihrer Realisierungsmittel meines Erachtens doch das historische Hörmaterial in besonderer Weise für einen Einsatz im Phonetikunterricht. Mit Hilfe des Hördokuments sollen die Lernenden auf ihrem Arbeitsblatt Pausen und Wortgruppenakzente markieren, denkbar wäre auch eine Markierung von Melodieverläufen. ${ }^{6}$ Im Anschluss an eine Kontrollphase im Plenum soll ein eigener Vortrag des Märchens vorbereitet und realisiert werden. Die Lerner müssen sich nicht in jedem Fall an die Pausen und Akzentuierungen Moissis halten. In jedem Fall aber sollte eine Aufnahme des jeweiligen Vortrags erfolgen, um eine Auswertung des Ergebnisses zu ermöglichen.

\section{Zusammenfassung und Schlusswort}

Historische Audio- und Videodokumente, das sollte in diesem Beitrag gezeigt werden, bieten über ihre landeskundlichen Lernmöglichkeiten hinaus Potenzial für den Deutsch-als-Fremdsprache- und Phonetikunterricht. Sie sind authentische Materialien, mit denen sich Hörfertigkeiten trainieren und Hörerfahrungen erweitern lassen. Sie können für phonetische und rhetorische Aspekte der Gestaltung gesprochener Sprache sensibilisieren, und das auch und gerade dort, wo sie durch ihre Distanz zu heutigen Sprech- und Hörgewohnheiten auffallen und dadurch Phänomene und Gestaltungsmittel besser erkennbar werden lassen. Auch Übungen zum Training produktiver Fertigkeiten, Sprech- und Ausspracheübungen lassen sich anschließen. Damit können die Materialien nicht nur einen für viele Lerner vereinfachenden, sondern nach Erfahrung der Verfasserin immer auch unterhaltsamen und motivierenden Zugang zur komplexen Materie phonetischer Gestaltung bieten.

\footnotetext{
6 Vorausgesetzt wird, dass die Studierenden mit Grundlagen der Prosodie des Deutschen wie den Regeln und Mitteln der Wort- und Satzakzentuierung, Melodieverläufen usw. vertraut sind.
} 


\section{Literatur}

Dieling, Helga; Hirschfeld, Ursula (2000): Phonetik lehren und lernen (Fernstudieneinheit 21). Berlin, München: Langenscheidt.

Endt, Ernst; Hirschfeld, Ursula (Hrsg.) (1995): Die Rhythmuslokomotive. München: Goethe-Institut.

Hirschfeld, Ursula; Neuber, Baldur (2010): Prosodie im Fremdsprachenunterricht Deutsch - ein Überblick über Terminologie, Merkmale und Funktionen. In: Deutsch als Fremdsprache, 1. Quartal 2010/Heft 1, 10-16.

Krech, Eva-Maria; Stock, Eberhard; Hirschfeld, Ursula; Anders, Lutz Christian (2009): Deutsches Aussprachewörterbuch. Berlin, New York: de Gruyter.

Müller, Beate (2004): Zu den Tonaufnahmen. In: Deutsches Historisches Museum/Deutsches Rundfunkarchiv, Frankfurt/Main - Potsdam-Babelsberg/ Institut für Sprechwissenschaft und Phonetik, Martin-Luther-Universität HalleWittenberg (Hrsg.): Der Klang der zwanziger Jahre. Reden Reportagen Rezitationen 1920-1930 (Booklet der CD).

Reinke, Kerstin (2008): Zur Wirkung phonetischer Mittel in sachlich intendierter Sprechweise bei Deutsch sprechenden Russen. (Hallesche Schriften zur Sprechwissenschaft und Phonetik Bd. 26). Frankfurt/Main: Peter Lang.

Stock, Eberhard (1996): Deutsche Intonation. Berlin, München: Langenscheidt.

\section{Quellen der Audio- und Videodateien}

Clairon, Elsa; Doutriaux, Claire (2005): les voeux/die Neujahrsansprachen. In: Doutriaux, Claire: Karambolage. Une émission franco-allemande ludique et impertinente/Eine deutsch-französische Sendung, frech und verspielt. DVD, Arte Video.

Die Prinzessin auf der Erbse: Deutsches Historisches Museum/Deutsches Rundfunkarchiv, Frankfurt/Main - Potsdam-Babelsberg/Institut für Sprechwissenschaft und Phonetik, Martin-Luther-Universität Halle-Wittenberg (Hrsg.) (2004): Der Klang der zwanziger Jahre. Reden Reportagen Rezitationen 1920-1930 (CD).

Mein kleiner grüner Kaktus: http://www.youtube.com/watch?v=qB1_DDv7iF0 (letzter Zugriff: 10.05.2011)

Weihnachtsansprache 2010: http:/ /www.youtube.com/watch?v $=\mathrm{qFaHVwJdQhk}$ (letzter Zugriff: 10.05.2011) 



\title{
Formen der Arbeiterliteratur für den Deutsch- und DaF-Unterricht
}

\author{
Jens Grimstein
}

\section{Einleitung}

Aktuelle literaturdidaktische Diskussionen beziehen zunehmend auch kulturwissenschaftliche Fragestellungen mit in ihre Überlegungen ein. ${ }^{1}$ Unter Berücksichtigung moderner Theorien eines erweiterten Textbegriffs wird das Medium Literatur verstärkt in seinen sozialen, anthropologischen und symbolsystematischen Dimensionen betrachtet. Literatur erfüllt angesichts ihres Einflusses auf Wahrnehmung und Verhalten Einzelner lebenspraktische Bedürfnisse und erweitert so ihre vormals begrenzte Stellung in der hermeneutischen Textwissenschaft. ${ }^{2}$

Mit diesen neuen Prämissen bietet sich ein literarisches Genre zur Didaktisierung im Germanistik- und/oder DaF-Unterricht an: die Arbeiterliteratur. Bevor im weiteren Verlauf konkrete Anwendungsbeispiele vorgestellt werden, soll zunächst theoretisch erörtert werden: Was bezeichnet Arbeiterliteratur? Welchen Stellenwert kann sie als historische Quelle beanspruchen? Und warum überhaupt eignet sich Arbeiterliteratur für den Germanistik- oder DaF-Unterricht?

\footnotetext{
${ }^{1}$ Vgl. Abraham; Kepser 2009: 10

2 Mit „Textwissenschaft“ ist die analytische Beschäftigung mit in diesem Fall literarischen Texten gemeint. Innerhalb eines Medienpluralismus der Humanwissenschaften wird damit der Literatur neben beispielsweise dem Film, der Musik oder der Fotographie ein gleichberechtigter Rang eingeräumt und jeweils jedes Medium innerhalb seiner eigenen Repräsentationsgrenzen bewertet.
} 


\section{Arbeiterliteratur}

In ihrer klassischen Ausprägung beschäftigt sich die Arbeiterliteratur ${ }^{3}$ mit der Darstellung von menschlichen Lebensverhältnissen innerhalb gesellschaftlich unterprivilegierter Schichten unter besonderer Berücksichtigung ihrer beruflichen Tätigkeiten. ${ }^{4}$ Ihre stärkste Prägung fand die Arbeiterliteratur in den historischen und sozialen Umwälzungen des 19. und 20. Jahrhunderts, denen sie einen Großteil ihrer Werke verdankt. Es sind vor allem die Arbeitsbedingungen des Industrieproletariats bis zum 2. Weltkrieg, die im Zentrum der Beschreibungen stehen. Auf diese Weise bildete sich der originär-industrielle Typus des Arbeiters bzw. der Arbeiterin heraus, der bis heute Einfluss auf politische Diskurse hat. In Opposition zum bürgerlichen Unternehmer (auch: Kapitalisten) definierte sich die Arbeiterliteratur über ihre Protagonisten, die sie auf verschiedenen Ebenen mit gesellschaftlichen Problemen konfrontiert. In der literarischen Praxis entwickelte sich so eine genrespezifische Poetik und Ästhetik der Arbeiterliteratur, die für die realen sozialpolitischen Veränderungen einen eigenen, mit einem politischen Impetus verbundenen künstlerischen Ausdruck fand. Gegenüber stärker kunsttheoretischen Bewegungen wie der Romantik oder dem Symbolismus ist es ein erklärtes Anliegen der klassischen Arbeiterliteratur, mittels literarischer Werke auf die soziopolitischen und ökonomischen Verhältnisse einzuwirken.

Dies gelingt ihr in unterschiedlicher Qualität vor allem in dem Zeitraum zwischen ca. 1850 und 1980, wobei ihre Hochphase in Europa zwischen 1880 und 1950 angesiedelt werden kann. Ihre thematischen Schwerpunkte fand sie für die deutschsprachige Literatur insbesondere in den Epochen des Naturalismus, in der Weimarer Republik/Sachlichkeit sowie in der Literatur der DDR. Mit ihrer deutlichen gesellschaftlichen Ausrichtung steht die Arbeiterliteratur so in Teilen der Aufklärung nah. Es geht ihr primär um die Emanzipationsbestrebungen einer sozial vernachlässigten Klasse, deren politisches Bewusstsein durch die materiellen Bedingungen nicht zur Entfaltung kommen kann. Eine historische und ideologische Nähe der Arbeiterliteratur zum Historischen Materialismus und später zur Kritischen Theorie zieht sich somit wie ein roter Faden durch die verschiedenen Epochen, an denen sie partizipiert (vgl. Scholz 2007: 41f.). ${ }^{5}$ Umso ironischer

\footnotetext{
${ }^{3}$ Die Prämissen der Arbeiterliteratur für die zweite Hälfte des 20. Jahrhunderts sollen hier ausdrücklich nur auf die Literatur der BRD bezogen verstanden werden. Die Arbeiterliteratur der DDR wird aus Gründen ihrer Vielfalt und ihrer politisch anders zu bewertenden Nähe zur staatlichen Doktrin hier ausgeschlossen, da dies einen eigenen Artikel verlangen würde, der auf angemessene Weise die DDR-Arbeiterliteratur und ihren Wert für den DaF- und Germanistikunterricht behandelt.

${ }^{4}$ Vgl. dazu auch Goette 1975: 2. - In diesem in der BRD erschienen Band enthält sich der Herausgeber einer Definition der Arbeiterliteratur und versucht stattdessen, die Texte für sich sprechen zu lassen.

${ }^{5}$ Diese Verbindung findet im Selbstverständnis der klassischen Arbeiterliteratur ihren vorläufigen Höhepunkt in den literarischen Werken der sozialistischen DDR, in der sie quasi als Hypostase ihres teleologischen Programms auftritt. Nicht zufällig forderte die Kulturpolitik des „Bitterfelder Weges" die schriftstellerische Tätigkeit der landwirtschaftlichen und industriellen Arbeiter, um im Gegensatz die Künstler zur materiellen Produktionstätigkeit zu verpflichten.
} 
nimmt sich die Tatsache aus, dass häufig die Autoren, die sich für die Belange der Arbeiter/-innen einsetzten, aus bürgerlichen Kreisen stammten und auch als Künstler diesem Milieu fest verhaftet blieben.

\section{Warum Arbeiterliteratur?}

\subsection{Allgemeine kulturelle Gründe}

Im Verlauf ihrer Entwicklung durchläuft die Arbeiterliteratur diverse Epochen, präsentiert sich in unterschiedlichen Gattungen und variiert vielfältig ihr Thema. Dadurch präsentiert sie ihren zentralen Begriff, die „Arbeit“, in seiner Diskontinuität und Wandelbarkeit und entfaltet ein beträchtliches historisches Reflexionspotential. Eines der Leitmotive, die Arbeitsbedingungen, aber auch „Arbeit“ selbst zeigen sich in ihren diachronen Differenzen und machen so auf Kontexte aufmerksam, die mithilfe moderner Theorie neu bewertet werden können, wie z.B. das Funktionieren ökonomischer Zusammenhänge im Vergleich zur Rechtsprechung oder die Polyphonie literarischer Stimmen über Arbeit. Damit bietet die Lektüre die Möglichkeit zur individuellen und kollektiven Sensibilisierung für die Verhältnisse der Gegenwart, aber auch für die Fragen nach einer gerechten Welt oder den (Un)Sinn von z.B. staatlich verwalteter Berufsausübung (oder Nichtausübung).

Darüber hinaus vermittelt die Arbeiterliteratur konkretes geschichtliches Wissen z.B. zu Gründungen und Entwicklungen politischer Parteien und Programme. Sie kann als ein Verbreitungsmedium soziohistorischer Ereignisse betrachtet werden, deren größere Kontexte sie notwendigerweise als klassenübergreifendes Phänomen mitthematisiert. Arbeiterliteratur vermag die Gesetzmäßigkeiten allgemeinen sozialen Wandels kritisch zu prüfen und auf Zukunftsfragen hin auszurichten. Mit dieser inhärenten pädagogischen Ausrichtung eignet sie sich insbesondere für aktuelle Debatten über die Abläufe in und um Ausbildungsinstitutionen wie Schulen oder Universitäten.

\subsection{Gründe für den Mutter- und Fremdsprachenunterricht}

Aus der oben beschrieben Ästhetik und Poetik der Arbeiterliteratur ergeben sich weitere theoretische Anschlüsse bezüglich ihrer Repräsentationseigenschaften. ${ }^{6} \mathrm{Mit}$ dem Anspruch, die soziale und politische Wirklichkeit für eine bestimmte soziale Gruppe verändern zu wollen, bedient sich die Arbeiterliteratur eines vielfältigen Inventars an literarischen Formen, denen gattungstechnisch bestimmte Funktionen zugeschrieben werden. Dazu gehören u.a. politische Lyrik, dramatische Texte,

${ }^{6}$ Dabei sollen ideologieaffine Stellungnahmen wie die Goettes zugunsten einer pluralistischen Perspektive auf die didaktischen und politischen Einflüsse vermieden werden, vgl. Goette 1975: 2f. 
autobiographische und satirische Schriften sowie journalistische Reportagen und allgemein Berichte. Ihre Textsortenvielfalt bietet für einen sprach- und textorientierten Germanistik- oder DaF-Unterricht eine Breite an schriftlichen Übungen und Ausdrucksformen, die für ein differenziertes, tiefschichtiges Verstehen (im Falle der Germanistik bei Muttersprachlern) und sprachorientiertes Lernen (im Falle des DaF-Unterrichts) relevant sind. Eine literarische Form kann hierbei unterschiedliche Fertigkeiten anregen, wenn z.B. anhand eines journalistischen Be von E.E. Kisch oder G. Wallraff beobachtendes und kommentierendes Schreiben geübt werden.

\section{Arbeiterliteratur als historische Quelle}

Mit dem Verweis auf Epochen und Entwicklungen innerhalb der Arbeiterliteratur wird eines ihrer Charakteristika hervorgehoben: Arbeiterliteratur kann neben einer synchronen, d.h. je nach Zeitabschnitt gegenwartsbezogenen Lektüre auch schwerpunktmäßig aus einem historischen Interesse gelesen werden. Diese Historizität bedarf einer Klärung ihres geschichtswissenschaftlichen Wertes, d.h. ihres Status als historische Quelle. Unter welchen Bedingungen kann die Arbeiterliteratur einen Beitrag für historische Studien leisten? Und was sind die Merkmale einer historischen Quelle?

\subsection{Primär- oder Sekundärquelle?}

Eine grundlegende Unterscheidung in der Geschichtswissenschaft betrifft die Klassifizierung eines Gegenstandes als Quelle ${ }^{7}$ (vgl. Jordan 2005: 49ff.). Man differenziert hierbei in der Regel zwischen Primär- und Sekundärquellen. Als Quelle gilt zunächst aus historischer Perspektive, was mittelbar oder unmittelbar mit der zur erforschenden Zeit in Verbindung steht. ${ }^{8}$ Inwiefern ein Text der Arbeiterliteratur aus Sicht einer Quellenklassifikation in Betracht kommt, hängt vom Untersuchungsgegenstand ab. Da sich meist das Interesse, sei es literaturwissenschaftlicher oder didaktischer Natur, auf den Text als solchen bezieht, gehört er zu den Primärquellen. Lediglich bei einem Untersuchungsgegenstand, bei dem ein

\footnotetext{
${ }^{7}$ Als Quelle kann prinzipiell alles gelten, was für das Verständnis eines historischen Sachbestands bedeutsam ist, also sowohl schriftliche Zeugnisse allgemein als auch auditive und visuelle Medien. Vgl. dazu auch die Beiträge von Marc Hieronimus (Einleitung) und Thomas Roth in diesem Band.

${ }^{8}$ Als Beispiele für den Unterschied Primärquelle (1) und Sekundärquelle (2) können dienen: 1) z.B. eine Reportage E.E. Kischs über die Stahlindustrie im Ruhrgebiet der 1920er Jahre, in welcher die journalistische Darstellung der Zustände interessiert, d.h. wie Kisch seinen Gegenstand beschreibt; 2) wenn besagte Reportage bei einer Untersuchung der realen Arbeitsbedingungen in den Stahlwerken im Ruhrgebiet als Kontext herangezogen wird, aber nicht ausschlaggebend für die Untersuchung ist. - Ein besonderer Fall dieser Quellenlage für die Literaturwissenschaft könnte „In Stahlgewittern" von Ernst Jünger darstellen, welches einerseits als authentisches Kriegstagebuch verfasst, anschließend aber in zahlreichen Überarbeitungen publiziert wurde.
} 
Text der Arbeiterliteratur assistierend für dessen Verständnis von Belang ist, wird von einer Sekundärquelle gesprochen.

Für die Arbeiterliteratur gilt in dieser Hinsicht, dass sie je nach Lese- und Forschungsinteresse, sofern es sich aus historischem Anliegen auf einen bestimmten Zeitraum bezieht, in beiden Fällen als Quelle für die damaligen Lebensbedingungen der Menschen gewertet werden kann. Ihre Motivation, der Wirklichkeit ein möglichst getreues Abbild zu verschaffen, steht hierbei in Spannung zu ihrem Status als fiktionalem Text. Die Arbeiterliteratur nimmt hier einen Sonderfall ein: Einerseits hat sie den Anspruch, die Arbeitswelt durch ihre literarischen Formen und ihre Bezugnahme auf reale Probleme empirisch abzubilden; andererseits teilt sie das Schicksal einer sich auch als Kunstform verstehenden Literatur, die bewusst ästhetisch Gebrauch von rhetorischen und narrativen Instrumenten macht. ${ }^{9}$ Damit verweisen die Werke der Arbeiterliteratur auf einen weiteren Zusammenhang in ihrer Funktion als historische Quelle.

\subsection{Kulturwissenschaftliche Quelle}

Der amerikanische Anglist Louis A. Monrose prägte einen Chiasmus, der die Beziehung von Geschichte und Literatur, d.h. Texten, auf den Punkt bringt: Die „Geschichtlichkeit von Texten und die Textualität von Geschichte“ (Monrose 2001: 67) verbindet ein genuin kulturwissenschaftliches Interesse zweier erkennt-

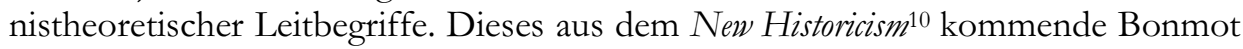
verweist auf die Rückführung literarischer Phänomene auf kulturelle Kräfte, denen sie wechselseitig entstammen. Es sind demnach besondere, von bestimmten Kontexten abhängige soziale Umstände, die in einem literarischen Text ihre signifikanten Spuren hinterlassen. Umgekehrt beeinflusst die Literatur wiederum ihre Kultur und damit die auf sie einwirkenden sozialen Bedingungen. Mag dieser Zusammenhang einer wechselseitigen Beeinflussung auch zunächst banal erscheinen, zeigt sich doch im Einzelfall die Unhintergehbarkeit dieses Gedankens. Gerade Werke, die der Arbeiterliteratur zuzurechnen sind, haben in besonderem Maße und zweifachem Sinn die „sozialen Energien“ (so ein Ausdruck Stephen Greenblatts) ihrer Zeit aufgenommen und künstlerisch verarbeitet, um wiederum Einfluss auf die Arbeiterbewegungen zu nehmen. Aus den Straßenkämpfen des

\footnotetext{
${ }^{9}$ So zeugen Brechts „Episches Theater“ der mittleren, marxistisch geprägten Werkphase oder Piscators experimentelles Agitprop-Theater eindrucksvoll davon.

${ }^{10}$ Mit der Erfolgsgeschichte der Foucaultschen Diskursanalyse entwickelten sich auch theoretische Ableger wie der besagte „New Historicism“. Berühmtester Vertreter ist der Literaturtheoretiker Stephen Greenblatt, der vor allem mit seinen Shakespeare-Studien großen Erfolg hatte (Greenblatt 1993). Leitgedanke des New Historicism ist vor allem die Annahme einer dynamischen Natur literarischer Werke und ihr reziprokes Verhältnis zu ihrer nächsten Umgebung, die maßgeblich an ihrer Entstehung beteiligt ist. - Als geschichtswissenschaftliches Pendant zum Zusammenhang Geschichte und Literatur kann Hayden Whites These von der Poetik der Geschichte gelten, wonach er Geschichtsschreibung in ihrer Eigenschaft als Narration untersucht, vgl. White 1991. Zur Verwendung kulturwissenschaftlicher Inhalte im Deutschunterricht siehe Raith 2009.
} 
Proletariats entstandene und später aufgezeichnete Lieder, von Arbeitern verfasste Autobiographien, Dramentexte und journalistische Texte, aber auch die Aneignung literarischer Werke für die eigene Sache geben hiervon Zeugnis.

Für eine geschichtswissenschaftliche Untersuchung kann die Arbeiterliteratur somit einen Beitrag dazu leisten, die Schnittstellen und Bündelungen von symbolischen Ordnungen, in denen sich sozioökonomische und politische Prozesse kreuzen, zu sammeln und hervorzuheben. Eine literarische Gattung stellt hierbei einen wortwörtlichen Kontext bereit, der das Verständnis historischer Phänomene erweitert. Dass ein solcher auch für konkrete Sprachvermittlungsmaßnahmen zur Verfügung steht, soll nun mit Beispielen verdeutlicht werden.

\section{Anwendungsbeispiele für Arbeiterliteratur im Germanistik- und DaF-Unterricht}

Im Folgenden werden für die oben beschriebenen Erläuterungen drei Unterrichtsbeispiele vorgestellt, um die didaktische Funktionalität der Arbeiterliteratur darzustellen. Ein besonderes Augenmerk soll dabei auf die Textsortendidaktik gelegt werden, d.h. das Üben und/oder Vertiefen bereits erlernter Kenntnisse anhand schriftlicher Aufgaben mit den gattungsdifferenten Texten der Arbeiterliteratur. Wichtig ist zu betonen, dass die einzelnen Übungen keine in sich abgeschlossene Unterrichtseinheit darstellen, sondern als anwendungsorientierte Einzelbeispiele gedacht sind, die sich, z.B. als Impulsgeber, in einen umfangreicheren Kontext „Arbeit“ oder „Arbeiterliteratur“ integrieren lassen. ${ }^{11}$ Hierbei bietet sich im Übrigen ein interdisziplinärer Austausch mit Nachbardisziplinen wie Geschichte oder Sozialwissenschaften an.

\subsection{Gegenstand}

Die Übungen decken die drei literarischen Hauptgattungen Lyrik, Drama und Epik ab, d.h. ein Gedicht, ein Theater- und ein Prosatext werden drei (literatur)historischen Epochen zugeordnet. Das Gedicht „Kinderlied für Arbeitslose“ wurde gegen Ende der Weimarer Republik vom Lyriker und Kinderbuchautor Erich Kästner verfasst. Der Dramenbeitrag des Nobelpreisträgers Gerhart Hauptmann ist ein Auszug aus seinem frühen naturalistischen Erfolgsstück „Vor Sonnenaufgang“ aus den späten 1880er Jahren. Kathrin Rögglas Prosatext „Wir schlafen nicht" aus dem Jahr 2006 kann der aktuellen Gegenwartsliteratur zugerechnet werden. ${ }^{12}$

11 Aus diesen Gründen wird auch auf eine tabellarische Unterrichtsübersicht, wie sie für Lehreinheiten üblich ist, verzichtet; es werden nur die didaktisch relevanten Kriterien am Anfang jeder Übung genannt.

${ }^{12}$ Literaturgeschichtlich hat sich für Texte aus diesem Zeitraum noch kein mehrheitsfähiges Epochenetikett durchsetzen können. Will man nichtsdestotrotz eine Epochenbezeichnung, dann bietet sich wohl das höchst undeutliche Kennwort „Postmoderne“ an. 


\subsection{Thema und Lernerinteresse}

Die drei genannten Textbeispiele stellen aufgrund ihrer Gattungsdifferenzen unterschiedliche didaktische und ästhetische Herausforderungen dar. Dramatische und epische Texte finden bei Lernern und Lehrern häufig eine stärkere Resonanz als lyrische Werke. Dennoch darf die Bedeutung lyrischer Formen nicht unterschätzt werden, wie schon Abraham und Kepser (2009: 150ff.) erläutern: Die drei alltäglichen Ausdrucksformen Kinderreim, Poetry Slam und (Pop-)Musik verwenden sie intensiv, darüber hinaus stellt aber die Lyrik eine oft gebrauchte Grundlage zur Darbietung der Arbeiterliteratur dar. ${ }^{13}$ Nicht minder zahlreich sind daneben die Prosawerke und Dramen der Arbeiterliteratur. Hier dürften also bei den drei Gattungen verschiedene Interessen seitens der Lehrenden und Lernenden bedient werden.

Lyrik, Drama und Prosa vermitteln daneben auch unterschiedliche Rezeptionsangebote, die sich wiederum aus didaktischer Sicht mit der Frage nach dem Bezug zur - auch beruflichen - Lebenswirklichkeit verbinden lassen. Anders gefragt: Welche für den (Arbeits)alltag eines Lerners notwendigen kommunikativen Fertigkeiten stellt die Auseinandersetzung mit Arbeiterliteratur zur Verfügung? Jede Textsorte setzt hierfür andere Schwerpunkte. Dramentexte fordern dialogisches Sprechen und szenisches Verhalten und bieten eine Grundlage für phonetische Aufgaben. Prosatexte bedienen alltägliche Lesegewohnheiten (man denke z.B. an Zeitungen oder Erläuterungen) und sind aufgrund ihrer Gattungsbreite (Essay, Bericht, Aufsatz, Kommentar usw.), zumal in einer Fremdsprache, ein nicht leicht zu beherrschendes Format. Lyrik verfügt formal und inhaltlich über Mittel, die den Rezipienten für sprachliche und rhythmische Qualitäten einer Sprache in seiner Sprachwahrnehmung sensibilisieren, indem hier „,mit wenigen Worten viel gesagt werden kann." 14 Dementsprechend verfolgen die Übungen das Ziel, die Stoffe über die rezeptionsästhetische und textgebundene Beschäftigung hinaus für lebenspraktische Bereiche nutzbar zu machen. Es sei dennoch bemerkt: Es handelt sich bei dieser Angelegenheit immer noch primär um eine literarische Ressource, die Impulse für eine produktions- und handlungsorientierte Didaktik geben kann. Lernerfolge bei der Beschäftigung mit Arbeiterliteratur können die kommunikativen Kompetenzen des Lerners sensibilisieren und im Idealfall bei einer späteren Berufsausübung unterstützend wirken. Dies allerdings gilt auch für viele andere Bereiche der (Fremd-)Sprachendidaktik.

\footnotetext{
${ }^{13}$ So schrieben Heinrich Heine und Georg Herwegh und später Brecht, Johannes R. Becher oder Wolf Biermann (neben den vielen Arbeiterliedern weniger prominenter Autoren) eine Vielzahl von Gedichten zum Thema Arbeit.

14 Abraham; Kepser 2009: 150.
} 


\subsection{Lerngruppe und Lernziel}

Die folgenden Aufgaben sind für eine Lerngruppe nach den GER-Kriterien des Niveaus B2 konzipiert. Zeit, Material und Sozialformen werden jeweils genannt. Die Begründung für die genannte Niveaustufe ist, dass diese Stufe für den Germanistikunterricht auch für Muttersprachler noch ausreichend Arbeitsanreize bietet, und in den meisten Fällen können die Aufgaben seitens der Lehrkraft ohne große Anstrengungen auf ein höheres Niveau gehoben werden, indem die Arbeitsanweisungen geringfügig verändert werden. Andererseits darf ein Niveau B2 bei Fremdsprachenlernern für den Abschluss einer Licence 3 (z.B. im Falle Frankreichs) vorausgesetzt werden; häufig aber lassen sich mit ebenfalls veränderter Arbeitsanweisung die Übungen leicht nach unten, d.h. auf Niveau B1 verlegen und so dem Lernerniveau anpassen. Niedrigere Niveaus als die genannten kommen für eine Nutzung nicht in Betracht, da ein angemessenes Verständnis literarischer Werke dort mit hoher Wahrscheinlichkeit nicht geleistet wird. ${ }^{15}$ Für den muttersprachlichen Unterricht beziehen sich die didaktischen Vorschläge vor allem auf den schulischen Bereich der Sekundarstufen I (Klassen 9 und 10) und II.

Jedem Textbeispiel wurden verschiedene Aufgaben beigefügt, die allerdings zusammen keinen thematischen oder didaktischen Zusammenhang bilden. Vielmehr sollen die Aufgaben einzeln für sich stehen und so für die Einzelstunde Material zum Thema bieten. Statt einer in sich geschlossenen Unterrichtseinheit zum Thema, die man idealerweise unter strukturell stabilen und regelmäßigen Lehrbedingungen durchführen kann, sollen die Einzelaufgaben dem Umstand Rechnung tragen, dass sich längere Unterrichtseinheiten gerade nicht immer problemlos in den Lehralltag integrieren lassen.

Als Lernziel sollen die Lerner bei der sprach- und formanalytischen Aufarbeitung anhand einer oder mehrer Textsorten zum Themenkomplex Arbeiterliteratur kulturelle Problemzusammenhänge kennen lernen und vor dem Hintergrund ihrer sprachlichen Kompetenz in einen neuen, sprachlich alltagspraktisch relevanten Zusammenhang transportieren können. Sie tun dies im Einzelnen, indem sie

- mit den historischen Texten ihr Weltwissen reaktivieren,

- anhand konkreter sprachlicher Formen Grammatik und Bedeutung des Textes untersuchen und

- die zentrale Thematik Arbeit in neue sprachlich fundierte Handlungsmuster ihrer Lebenswirklichkeit überführen.

\subsection{Unterrichtsbeispiele}

Folgende drei Übungen werden exemplarisch für eine Nutzung der Arbeiterliteratur im $\mathrm{DaF}$ - und Germanistikunterricht beschrieben:

${ }^{15}$ Für Beispiele dieser Art vgl. Abraham; Kepser 2009: 150-160. 
Prosa - Kathrin Röggla: „Wir schlafen nicht“ (Auszug)

Lyrik - Erich Kästner: „Kinderlied für Arbeitslose“

Drama - Gerhart Hauptmann: „Vor Sonnenaufgang“ (Auszug)

\section{Röggla, ,Wir schlafen nicht"}

Textauszug: ${ }^{16}$

Reinkommen (die Praktikantin)

„Und wieder kennen sich andere aus mit Vorständen und Aufsichtsräten.“ Und wieder sei sie nicht dabei. Ihr sei immer noch keine Firmenvergangenheit zur Hand, obwohl sie alles versucht habe. Dabei wolle sie doch gute PR-Arbeit machen, dabei wolle sie doch irgendwann ein guter Coach sein oder Kunden gut beraten, „was weiß ich“. Heute müsse man eben für alles offen sein, müsse man sich auf alles einstellen können.

Ja, so eine Verlagsvergangenheit würde sie gerne zusammenbringen wie Frau Mertens, oder eine Medienvergangenheit wie Frau Bülow, oder eine Beratervergangenheit wie Herr Bender. Aber von einer Pressestelle könne man heute nur noch träumen, so von einer Fixanstellung mit vernünftigem Gehalt. Nur noch träumen könne man von einer Stelle in einer Agentur und mitgeträumt sei dann immer auch schon eine kleine Vergangenheit, ein kleiner Erfahrungsschatz, den man austauschen kann.

Aber die anderen hätten ja alle Eltern. Die anderen hätten ihre Steuerberatereltern und Wirtschaftsprüfereltern, bei denen sie ein und aus gingen und die ihnen bezahlte Praktikumsplätze und Volontariate verschafften. Die anderen hätten ihre Eltern, so besorgende, besorgte und übersorgte, und sie habe eben keine Eltern. Zumindest nicht in dem Sinn, also keine Steuerberater-, keine Wirtschaftsprüfer-, und keine Unternehmensberatereltern. Oder Zahnarzteltern. Kleinbürgereltern, das ja, das könne man schon sagen, also praktisch nicht existierende, zumindest, was ihre berufliche Situation betreffe.

In Wirklichkeit brauche man wieder richtige Eltern. Das müsse sie jetzt einmal laut sagen, aber das glaube ihr niemand, genauso wenig wie jemand glaube, dass sie keine Krankenversicherung hat.

Z.B. ihre Eltern verstünden das nicht. Die würden immer nur sagen, es müsste doch möglich sein, einen Job zu finden, bei dem man Geld verdienen könne und nicht nur ein unbezahltes Praktikum.

${ }^{16}$ Röggla 2006: 87-92. - Für den vorliegenden Textauszug wurden behutsame Veränderungen im Originaltext zur eindeutigeren didaktischen Aufbereitung unternommen: Benutzung der Groß- und Kleinschreibung sowie Ersetzung des Konjunktivs durch den Indikativ. Auch sind einzelne Sätze, kürzere Satzteile oder kürzere Paragraphen weggelassen worden und stattdessen durch die Absätze angegeben. Zur Vollständigkeit wird auf den Originaltext verwiesen. 
Außerdem wolle sie ja gar nicht ins Consulting-Business hinein, da habe man sie schon richtig verstanden, sie habe da auch nichts verloren, aber woanders sei kein Job zu holen gewesen. Ja, was solle sie schon groß sagen, das ist nur ein Idiotenjob, den sie hier mache, und selbst der sei unbezahlt.

Nein, sie wisse nicht, zu was sie bereit wäre, um einen Job zu kriegen, das wisse sie nicht, sie vermute mal, zu einigem.

Glossar:

der Vorstand (Pl. die Vorstände): eine Kommission von Personen, die ein Unternehmen führen, z.B. ein Direktor.

der Aufsichtsrat (Pl. die Aufsichträte): eine Kommission in einem Unternehmen, die den Vorstand kontrolliert.

die PR-Arbeit (Public Relations): Kommunikation einer Firma mit der Öffentlichkeit.

der Berater (Pl. die -,er): ein Angestellter einer Firma, der einem Unternehmen mit wirtschaftlichen Problemen hilft.

der Steuerberater (Pl. die -,er): jemand, der Unternehmen und Privatpersonen bei Steuerfragen hilft.

der Wirtschaftsprüfer (Pl. die -,er): jemand, der die wirtschaftliche Existenz einer Firma kontrolliert.

Aufgabe 1:

Zeit: $\quad$ ca. 10 Minuten

Sozialform: Einzelarbeit

Lernziel: Grammatische Formen der direkten und indirekten Rede

Fassen Sie die Aussagen der Praktikantin zusammen. Benutzen Sie dafür die indirekte Rede und setzen Sie die konjunktivischen Verbformen in den Indikativ Präsens. Variieren Sie die Verben, die den Relativsatz einleiten. Bsp.: „.Ihr sei immer noch keine Firmenvergangenheit zur Hand, obwohl sie alles versucht habe“ wird zu „Sie sagt, dass ihr immer noch keine Firmenvergangenheit zur Hand ist, obwohl sie alles versucht hat."

Aufgabe 2:

Zeit: ca. 40 Minuten

Sozialform: Partnerarbeit

Lernziel: $\quad$ freies Sprechen, einen Dialog führen, Ratschläge geben

Entwerfen Sie stichwortartig Sprechhandlungsanweisungen für einen drehbuchartigen Dialog mit einem Teilnehmer und verteilen sie folgende Rollen: 1. die Rolle der Praktikantin; 2. die Rolle einer Freundin/eines Freundes. 
Die Freundin/der Freund soll in einem frei gesprochenen Dialog der Praktikantin persönliche Ratschläge für ihre Situation geben. Wiederholen Sie im Dialog auch Aussagen zu Ratschlägen wie „Überleg doch mal, X oder Y zu machen“; „Du könntest/du solltest...“; „Geh doch einfach mal zu/zum...“, „Wie findest du denn die Idee, ....“; Hast du schon an X gedacht?“; „Ich würde dir raten,....“

Bsp.: Praktikantin: (Stichwort Sprechhandlungsanweisung: „unsicher, nachdenklich"): „Irgendwie ist alles etwas komisch zur Zeit."

Freundin: (auf sie eingehend, interessiert): „Was ist los? Warum machst du so einen traurigen Eindruck? “

Aufgabe 3:

Zeit: $\quad$ ca. 10 Minuten

Sozialform: Einzelarbeit, auch Partnerarbeit möglich

Lernziel: $\quad$ sprachliche Abstraktion von einem Text in Form von Stichworten

Extrahieren Sie in Stichworten die Haltung der Protagonistin zur Arbeitswelt. Bsp.: „Ihr sei immer noch keine Firmenvergangenheit zur Hand, obwohl sie alles versucht habe“ wird zu ,kein Glück bei der Jobsuche, trotz großer Motivation“.

Aufgabe 4:

Zeit: $\quad$ ca. 60 Minuten

Sozialform: Partnerarbeit und anschließend Gruppenarbeit

Lernziel: $\quad$ freies Sprechen, Erstellen/Verbalisieren von Argumenten

Katrin Rögglas Textausschnitt zeigt nicht nur die Zweifel einer jungen Frau an ihren Fähigkeiten, sondern auch den mangelnden Glauben an einen Arbeitsmarkt, der Berufseinsteigern kaum noch reale Chancen einräumt, sich durch gute Leistung beruflich zu etablieren oder aufzusteigen.

Aber es gibt sicherlich auch Menschen, die z.B. in freien oder kreativen Berufen tätig sind und die ihre Arbeit mögen. Die Aufgabe ist es, als Gruppe in einem freien Vortrag den anderen Teilnehmern den Beruf zu empfehlen und positiv darzustellen. Bilden Sie also fünf Gruppen à zwei bis drei Lerner und übernehmen Sie folgende berufliche Rollen: ${ }^{17}$

Gruppe 1 = Angestellte(r) der Arbeitsagentur/Arbeitsvermittlung,

Gruppe 2 = Coaches,

Gruppe 3 = Kundenberater,

Gruppe 4 = PR-Fachmann,

${ }^{17}$ Berufsbezeichnungen sollten hier ggf. von der Lehrkraft vorher erklärt und evtl. anhand von Beispielen erläutert werden, z.B. mit Beschreibungen dieser Berufe von Seiten der Arbeitsagentur oder der entsprechenden Verbände. 
Gruppe 5 = Jury.

Notieren Sie nun in ihrer Gruppe (Ausnahme: die Jury!) Argumente für ihre Rolle, d.h. für den Beruf als Coach, Kundenberater, Angestellter der Arbeitsagentur oder PR-Fachmann. Bsp.: „Ich arbeite als Coach, weil ich die Fähigkeiten einer Person im Beruf fördern möchte“/„Ich arbeite als PR-Fachmann, weil mich Kommunikation und Öffentlichkeit interessieren." Präsentieren Sie nun in Gruppe 1 bis 4 nacheinander ihre Argumente so überzeugend wie möglich vor der Jury. Die JuryGruppe soll am Ende anhand der besten Argumentationen die Gewinnergruppe aussuchen.

\section{Kästner, „Kinderlied für Arbeitslose“}

Text: ${ }^{18}$

1)

Schlafzimmer habt ihr immer noch keins.

Doch Kinder kriegt ihr fast jedes Jahr eins.

Warum ihr das wohl tut?

Euch geht's wohl zu gut?

Der letzte Groschen wird verfeuert.

Der Vater wird bald ausgesteuert.

Das Hinterhaus ist voll Geschrei.

Eia popeia, eia popeia -

Von wegen Eiapopei!

2)

Die Dummheit sollte Grenzen haben.

Was sollen denn die vielen Knaben?

Sie werden erstens groß

Und zweites arbeitslos.

Wann werdet ihr denn nur gescheit?

Ihr seid nicht mehr, je mehr ihr seid!

Was soll die ewige Fortpflanzerei?

Eia popeia, eia popeia -

Von wegen Eia popei!

18 Kästner 1987: 43-44. 
3)

Und jeder hat Töchter, und jeder hat Söhne.

Und immer tiefer drückt man die Löhne.

Lasst doch die Kindereien!

Begnügt euch mit einem und zweien.

Ihr seid der Bund der Kinderreichen.

Ihr liefert für die Zukunft Leichen.

Ihr liefert dem Elend frei ins Haus.

Eia poeia, eia popeia -

Schlaft ein? Nein! Schlaft aus!

Glossar:

Groschen: früheres Zahlungsmittel vor der Einführung der D-Mark 1949.

Aussteuern: hier: jemandem kündigen und ihm einen letzten Geldbetrag auszahlen.

Eia poeia: Reim aus einem Kinderlied.

Leiche: ein toter Körper.

Aufgabe 1:

Zeit: $\quad$ ca. 20 Minuten

Sozialformen: Einzelarbeit

Lernziel: $\quad$ Bewerten und Begründen von Aussagen

Sehen Sie sich die Überschriften an. Welche Meldungen passen Ihrer Meinung nach am besten zu dem Gedicht? Begründen Sie ihre Wahl. Verwenden Sie auch mindestens einmal kausale Konjunktionen (weil, da) oder Präpositionen-NomenKonstruktionen (aufgrund/wegen + Genetiv; aus/vor + Dativ) und achten sie auf die Position des Verbs.

„FAZ“ (Februar 2007): Schulessen für Hartz-IV-Kinder

„BILD-Zeitung“ (Mai 2009): Kinderkriegen sollte nicht durch Sozialleistungen lukrativ gemacht werden

„Thüringer-Allgemeine Online“ (Juli 2010): Debatte um kostenlose Verhütung für Hartz-IV-Empfänger

„Merkur Online“ (Januar 2010): Von der Leyen besteht auf KindergeldRückzahlung von Hartz-IV-Empfängern

„Der Tagesspiegel Online“ (Juni 2010): Dann lieber Abtreiben. Kinder kriegen trotz unsicherer Jobs?

„Handelsblatt“ (Juli 2010): Haushalte mit Kindern sollten nicht mehr Geld bekommen. Allerdings über Gutscheine und Direktzahlungen Schul-Essen für Kinder

„Die Welt Online“ (Dezember 2010): Familien brauchen wieder mehr Planungssicherheit 
Aufgabe 2:

Zeit:

Sozialform: $\quad$ Einzelarbeit, die auch Partnerarbeit sein kann/darf

Lernziel: $\quad$ Erstellung eines tabellarischen Lebenslaufs, poetikaffine Übungen (Produktion von Elfchen, eines Hip-Hop-Textes)

1. Wer könnte die Person hinter den Zeilen 1-7 jeder Strophe sein, an die sich der Sprecher des Gedichts wendet? Aus welchem sozialen Umfeld kommt er/sie? Was für einen Beruf könnte er/sie haben? Ist er/sie arbeitslos? - was für eine Arbeit sucht die Person? Entwickeln Sie schriftlich einen tabellarischen Lebenslauf. ${ }^{19}$

2. Notieren Sie am (von der Lehrkraft vorher an die Tafel gezeichneten!) Wortigel ihre spontanen Assoziationen zum Thema „Arbeit“ und „Arbeitslosigkeit“. Schreiben Sie anschließend ein Elfchen in Partnerarbeit. Ein Elfchen ist ein Gedicht, in dem es höchstens elf Wörter gibt und in deren

1. Zeile - ein Wort

2. Zeile - zwei Wörter

3. Zeile - drei Wörter

4. Zeile - vier Wörter

5. Zeile - ein Wort

stehen/steht.

3. Verwandeln Sie schließlich das Elfchen in einen rhythmischen Text, d.h. ein Gedicht oder einen Hip-Hop-Song, und erzählen Sie darin die Kurzbiographie Ihrer Person aus Aufgabe 1 nach.

\section{Hauptmann, „Vor Sonnenaufgang“}

Textauszug (Dritter Akt): ${ }^{20}$

HOFFMANN: Sag mal, Loth, was führt dich eigentlich in unsere Gegend?

Ich hab' bisher ganz vergessen, dich danach zu fragen.

LOTH: Ich möchte die hiesigen Verhältnisse studieren.

HOFFMANN: Bitte...? ... Was für Verhältnisse?

LOTH: Präzise gesprochen: Ich will die Lage der hiesigen Bergleute studieren.

HOFFMANN: Ach, die ist im allgemeinen doch eine sehr gute.

\footnotetext{
19 Musterbeispiele und Konzeption tabellarischer Lebensläufe sollten je nach Lernervorwissen vor der Übungsdurchführung eingeführt werden.

${ }^{20}$ Hauptmann 1999: 70-72. Das Stück würde 1889 in Berlin uraufgeführt und veröffentlicht.
} 
LOTH: Glaubst du? - Das wäre ja übrigens recht schön... Doch eh ich's vergesse: du musst mir dabei einen Dienst leisten. Du kannst dich um die Volkswirtschaft sehr verdient machen, wenn...

HOFFMANN: Ich? I! wieso ich?

LOTH: Nun, du hast doch den Verschleiß der hiesigen Gruben?

HOFFMANN: Ja! und was dann?

LOTH: Dann wird es dir auch ein leichtes sein, mir die Erlaubnis zur Besichtigung der Gruben auszuwirken. Das heißt: ich will mindestens vier Wochen lang täglich einfahren, damit ich den Betrieb einigermaßen kennenlerne.

HOFFMANN: Was du da unten zu sehen bekommst, willst du dann wohl schildern?

LOTH: Ja. Meine Arbeit soll vorzugsweise eine deskriptive werden.

HOFFMANN: Das tut mir nun wirklich leid, mit der Sache habe ich gar nichts zu tun. - Du willst bloß über die Bergleute schreiben, wie?

LOTH: Aus dieser Frage hört man, dass du kein Volkswirtschaftler bist.

HOFFMANN: Bitte sehr um Entschuldigung. Du wirst mir wohl zutrauen... Warum? Ich sehe nicht ein, wieso man diese Frage nicht tun kann? - und schließlich: es wäre kein Wunder... Alles kann man nicht wissen.

LOTH: $\mathrm{Na}$, beruhige dich nur, die Sache ist einfach die: wenn ich die Lage der hiesigen Bergarbeiter studieren will, so ist es unumgänglich, auch alle Verhältnisse, welche diese Lage bedingen, zu berühren.

HOFFMANN: In solchen Schriften wird mitunter schauderhaft übertrieben.

LOTH: Von diesem Fehler gedenke ich mich freizuhalten.

Glossar:

hiesig: hier befindlich, ansässig

Grube: ein Bergbau

Verschleiß: Verbrauch

schauderhaft: schrecklich, sehr

Aufgabe 1:

Zeit:

Material: $\quad$ ggf. Computer mit Internetzugang \& Email-Account des Lerners

Sozialform: Einzelarbeit

Lernziel: $\quad$ Schriftliches Erstellen einer Kurzbiographie

Suchen Sie sich einen Partner und verteilen Sie die Rollen untereinander. Entwerfen Sie auf Grundlage der vorliegenden Zeilen für ihre Rolle eine Kurzbiographie (Name, Alter, Herkunft, Studium/Ausbildung, Familie, wichtige Reisen, 
Berufserfahrungen, persönliche Interessen und anderes). ${ }^{21}$ Lesen Sie anschließend mit einem Partner zusammen die Szene in ihren jeweiligen Rollen. Überlegen Sie außerdem: Wie würden Sie ihre Figur stimmlich klingen lassen?

Aufgabe 2:

Zeit: ca. 15 Minuten

Sozialform: Einzelarbeit

Lernziel: Schreiben einer offiziellen Email

Angenommen, die Szene würde in der Gegenwart spielen: Formulieren Sie eine Email $^{22}$ aus der Sicht des Chefs (= Herr Hoffmann) an seine Mitarbeiter, in der Sie die Belegschaft über den Studienbesuch des Volkswissenschaftlers (= Dr. Alfred Loth) informieren. Bringen Sie dabei auch Hoffmanns Zurückhaltung als Firmenchef gegenüber dem Besuch in der Email unter.

Aufgabe 3:

Zeit:

Sozialform:

ca. 45 Minuten

Lernziel: Einzelarbeit

Lese- und Textverständnis, schriftlicher Ausdruck und schriftliches Bewerten von Texten

Die folgenden drei Auszüge sprechen jeweils von „Arbeit“. Nennen Sie schriftlich die Unterschiede der drei Texte zueinander (ca. 250 - 350 Wörter).

Die Figur Loth sagt im Hauptmann-Text den Satz „,...du hast doch den Verschleiß der hiesigen Gruben“. Was meint er damit? Auf welchen der drei folgenden Texte trifft der Satz am meisten zu? Begründen Sie ihre Wahl schriftlich.

Lesen Sie die drei Texte:

1. Programm der Sozialdemokratischen Partei Deutschlands. (Erfurt 1891, Auszug)

Die ökonomische Entwicklung der bürgerlichen Gesellschaft führt mit Naturnothwendigkeit zum Untergang des Kleinbetriebes, dessen Grundlage das Privateigenthum des Arbeiters an seinen Produktionsmitteln bildet. Sie trennt

${ }^{21}$ Beispiele und Übungen zu Kurzbiographie bzw. Kurzporträt (Name, Geburtsdatum, Herkunft, Ausbildung, wichtige Stationen im Leben etc.) sollten dieser Übung in einer anderen Lehreinheit vorgeschaltet werden, d.h. in einer Stunde vor Anwendung der obigen Übung bereits durchgenommen worden sein, damit die Lerner sie durchführen können.

22 Dasselbe gilt für die Email. Hier sollten die Lerner vor allem auf die verschiedenen Varianten (formell-informell) einer Email hingewiesen und daran Lernelemente wie z.B. die korrekte Anrede (Sehr geehrte(r), Guten Tag, Hallo usw.), sachgerechte Beschreibung des Anliegens oder Verabschiedung (Mit freundlichen Grüßen, Herzliche Grüße, Ciao usw.) je nach sozialem Kontext eingeübt werden. 
den Arbeiter von seinen Produktionsmitteln und verwandelt ihn in einen besitzlosen Proletarier, indeß die Produktionsmittel das Monopol einer verhältnismäßig kleinen Zahl von Kapitalisten und Grundbesitzern werden. Hand in Hand mit dieser Monopolisirung der Produktionsmittel geht die Verdrängung der zersplitterten Kleinbetriebe durch kolossale Großbetriebe, geht die Entwicklung des Werkzeugs zur Maschine, geht ein riesenhaftes Wachsthum der Produktivität der menschlichen Arbeit. Aber alle Vortheile dieser Umwandlung werden von den Kapitalisten und Großgrundbesitzern monopolisirt. Für das Proletariat und die versinkenden Mittelschichten - Kleinbürger, Bauern bedeutet sie wachsende Zunahme der Unsicherheit ihrer Existenz [...].

2. Max von der Grün, „Irrlicht und Feuer“ (1963, Auszug)

So ist das also. Eineinhalb Jahrzehnte tat ich meine Arbeit recht und schlecht, war pünktlich und zuverlässig. Aber dann wird man plötzlich zur schiefen Nummer in der dreitausendköpfigen Belegschaft, nur weil man zweimal unentschuldigt gefehlt hat. [...] Menschliche Schwächen haben in einem modernen Industriebetrieb nichts zu suchen, da wird mit Psychologen Kalkulation gemacht, mit Stoppuhren die Produktion errechnet.

\section{Ralf Dahrendorf: „Neue Weltordnung“ (1997, Auszug)}

Die Frage ist also: Was tun Menschen mit ihrer Zeit, woher kriegen sie ihren Lebensunterhalt und ihr Selbstbild? Das wird in den nächsten 100 Jahren anders aussehen als in den letzten 100 Jahren. Vor 100 Jahren gab es keine Arbeitslosigkeit, sie ist eine Erfindung des späten 19. Jahrhunderts. Vorher hatten Menschen kompliziertere Leben. Auch die Industriearbeiter hatten meist noch eine Beziehung zum Land oder zu anderen Lebenstätigkeiten. ${ }^{23}$

\section{Zusammenfassung}

Texte der Arbeiterliteratur besitzen aufgrund ihrer lebensnahen Problematisierung eines universalen Gegenstandes ein großes Potential für die Darstellung historischer Prozesse im Hinblick auf Themen aus der Arbeitswelt. Die gegenwärtigen globalen Dynamiken von Arbeitsgesellschaften richten das Interesse auch auf die geschichtlichen Entwicklungen von Arbeit, die exemplarisch auf dem Gebiet der (Arbeiter)literatur ihren Niederschlag finden. Sie ermöglicht dem Leser dadurch ein breiteres Verständnis der Bedingungen, unter denen sich bis heute Arbeit als Diskurs präsentiert. Die Arbeiterliteratur reflektiert texthermeneutische Modelle der Kulturwissenschaften und wird so doppelt lesbar: Einerseits als literarischer Text, andererseits als schriftliche Verkörperung konkreter sozialer Umstände der Arbeitswelt. Dies drückt sich nicht zuletzt auch in der Wahl ihrer Textsorten aus,

${ }^{23}$ Vgl. Beck 2007: 126. 
die vom fiktionalen Text über die Autobiographie bis zur dokumentarischen Darstellung reicht.

Unter Berücksichtigung dieser Prämissen bietet sich die Arbeiterliteratur in besonderem Maße für didaktische Bearbeitungen und Benutzungen an, da sie in ihren Übungen sowohl bereits bestehendes als auch neues Wissen von Lernern aktiviert. ${ }^{24}$ Zudem bildet sie auf der Basis spracherwerbs- und/oder sprachanwendungsorientierter Übungen Kompetenzen aus, die für einen späteren Berufsalltag von Interesse sein können. Die Beispielübungen sollen einen Eindruck von den Möglichkeiten eines didaktischen Transfers zwischen Literatur, Unterrichtsanwendung und imaginierter Berufswelt vermitteln. Dass an ihnen wie auch an anderen Übungen dazu noch gearbeitet werden kann, versteht sich von selbst.

\section{Literatur:}

Abraham, Ulf; Kepser, Matthis (2009): Literaturdidaktik Deutsch. Eine Einführung. Berlin: Erich Schmidt.

Beck, Ulrich (2007): Schöne neue Arbeitswelt. Frankfurt/Main: Suhrkamp.

Goette, Jürgen W. (1975): Arbeiterliteratur. Texte und Materialien zum Literaturunterricht. Frankfurt/Main: Diesterweg.

Greenblatt, Stephen (1993): Verhandlungen mit Shakespeare. Innenansicbten der englischen Renaissance. Frankfurt/Main: Fischer.

Greenblatt, Stephen (1995) Kultur. In: Baßler, Moritz (Hrsg.) (1995): New

Historicism. Literaturgeschichte als Poetike der Kultur. Frankfurt/Main: Fischer, 48-59.

Hauptmann, Gerhart (1999): Vor Sonnenuntergang. Berlin: Ullstein.

Jordan, Stefan (2005): Einfïhrung in das Geschichtsstudium. Stuttgart: Reclam.

Kästner, Erich (1987): Gedichte. Stuttgart: Reclam.

Monrose, Louis A. (1995): Die Renaissance behaupten. Poetik und Politik der Kultur. In: Baßler (Hrsg.) (1995), 60-93.

Sarter, Heidemarie (2006): Einführung in die Fremdsprachendidaktik. Darmstadt: WBG.

Scholz, Rüdiger (2007): Arbeiterliteratur. In: Burdorf, Dieter; Fasbender, Christoph; Moenninghoff, Burkhard (2007): Metzler Lexikon Literatur. Stuttgart: Metzler, 41-42.

Raith, Markus (2009): Vom Marmorbild zur Venus von Samoa. Kulturwissenschaftliche Perspektiven für die Deutschdidaktik. München: kopaed.

\footnotetext{
${ }^{24}$ Zum Einsatz von Literatur im Fremdsprachenunterricht zwischen Sprachrezeption und Sprachproduktion vgl. Sarter 2006: 94-99.
} 
Röggla, Kathrin (2006): Wir schlafen nicht. Frankfurt/Main: Fischer.

Von der Grün, Max (1963): Irrlicht und Feuer. Recklinghausen: Klartext.

White, Hayden (1991): Metahistory. Die historische Einbildungskraft im 19. Jahrhundert. Frankfurt/Main: Fischer. 



\title{
Namen schildern: Straßennamen und andere Namensfelder im DaF-Unterricht
}

\author{
Arndt Kremer
}

\section{Einleitung}

Forschungsansätze, die Fragen nach Identität und Mentalität mit kulturellen Raumkonzepten verbinden, haben Konjunktur. Vielleicht ist dies der Grund dafür, dass gerade kulturwissenschaftliche Reflexionen zu Straßennamen derzeit so en vogue sind. Lange Zeit eher heimatkundlich-anekdotisch ambitioniert, hat die Straßennamenforschung sich Ende der 1980er Jahre verstärkt kulturhistorisch-mentalitätsgeschichtlichen Erkenntniszielen gewidmet. So wurde dem Gedanken Rechnung getragen, dass Straßennamen nicht nur eine spezifische Orientierungs-, sondern auch eine ausgeprägte Erinnerungsfunktion haben, weshalb sie für die mnemische Interaktion und Kommunikation eine zentrale Rolle spielen. Besonders durch die Ergebnisse der Forschungsgruppe um den Kölner Sprachwissenschaftler Dietz Bering stehen der kulturwissenschaftlich interessierten Straßennamenforschung nun auch immer feiner justierte systematische Analyseraster zu vollständigen Korpora zur Verfügung. ${ }^{1}$

Dass sich die Bennennungspraxis von Straßennamen im Verlauf der Geschichte signifikant verändert hat und weiter verändern wird, und dies eben nicht

\footnotetext{
${ }^{1}$ Der erste Projektentwurf zu Straßennamen in Köln ist rückblickend beschrieben bei: Bering 2001: 270-281. Dann, fortführend, bei: Bering; Großsteinbeck 1994: 97-117; Bering; Großsteinbeck; Werner 1999: 135-166; Bering; Großsteinbeck 2007: 311-335. Neben Köln sind mittlerweile auch andere deutsche Großstädte mit günstiger Quellenlage gut untersucht, z.B. Magdeburg: Föllner; Luther; Weinert 2011.
} 
willkürlich, sondern aufgrund bestimmter gesellschaftlich-kulturell bedingter Wandlungen, ist eines der spannendsten Phänomene der Straßennamenforschung. Gerade hier bietet sich eine Didaktisierung des Themas im DaF-Bereich an, weil der Sprachunterricht durch landeskundliche und interkulturelle Fragestellungen bereichert werden könnte, deren Antworten die Lebenswelt der Lernenden betreffen, weniger pädagogische Legitimationsprobleme haben und für kognitive Lernprozesse sehr förderlich sind. Die kontrovers, ja oft hoch emotional geführten Bennennungsdebatten zu Straßennamenbenennungen und -umbenennungen sind historische Quellen, die höchst anschaulich vermitteln, dass dieses Feld keine quantité négligeable einer linguistischen Randdisziplin darstellt, sondern den Kern individueller und kollektiver Identitätsauslegungen trifft.

Zunächst werden im theoretischen Teil Straßennamen linguistisch verortet und ihre kulturhistorische Bedeutung erläutert, dann diachrone und synchrone Analyseergebnisse am Beispiel des mittelalterlichen Kölns präsentiert und schließlich die neuzeitlichen Straßennamen in Köln seit Anfang des 19. Jahrhundert beleuchtet. Im zweiten fachdidaktischen Teil wird aufgezeigt, inwiefern vor dem Hintergrund dieser kulturgeschichtlichen Erkenntnisse die Verwendung von Straßennamen, aber auch anderer Namensphänomene wie Objektnamen (hier: Namen von Fußballstadien) sinnvoll sein kann für den DaF-Unterricht. Abschließend werden in einer kontrastiv-interkulturellen Perspektive Straßennamen in einem anderen europäischen Land, in diesem Fall Malta, untersucht.

\section{Systematische Grundlagen}

\subsection{Die kulturhistorische Bedeutung von Straßennamen}

Namen stiften und sichern Identität, sie bilden feste Ankerpunkte, die Orientierung verschaffen. Das gilt für Eigennamen, aber auch für Straßennamen, den so genannten Hodonymen (von griech. hodos $=\mathrm{Weg}$ ), die als Teilgebiet der Namensforschung (Onomastik) zu den Örtlichkeitsnamen (Toponymen) gehören. Straßennamen zählen linguistisch zur Kategorie der Eigennamen (Nomen proprium), grenzen sich insofern von der größeren Gruppe der Gattungsnamen (Nomen appellativum) ab. Auf der denotativen Ebene bewegen sie sich jedoch auf einer gleitenden Skala zwischen beiden Nomina, indem ihr Bestimmungswort sowohl proprialen (Adenauerallee) als auch appellativen Ursprungs (Eichenstraße) sein kann. Straßennamen setzen sich zumeist aus einem Bestimmungswort (Hölderlin-) und einem Grundwort (-Straße) zusammen, besonders bei Toponymen (Flur, Ortsnamen) oft auch in Verbindung mit Präpositionalbildungen (An der Ling).

Auf semiotischer Ebene bilden Straßennamen Signifikanten, die entsprechend der Unterteilung nach Charles Sanders Peirce (1903) - im Sinne von Icons ein Ähnlichkeitsverhältnis (Am Hügel) zum Bezeichneten oder als Indizes ein Folgeverhältnis bzw. eine Hinweisfunktion (Platz, der deutschen Einheit) anzeigen 
können. Dabei haben Straßennamen zunächst einmal eine Primärfunktion: Sie dienen der Orientierung im Raum. Dass die Straße, die direkt an der Universität vorbeiführt, den Namen Universitätsstraße trägt, verwundert niemanden. Der Name erklärt sich von selbst. Straßennamen wären jedoch einseitig interpretiert, würde man ihre Bedeutung auf diese Primärfunktion beschränken. Der Name Universitätsstraße transportiert darüber hinaus noch etwas anderes, etwas, das bei dieser rein funktionalen Bezeichnung immer impliziert ist. Er zeigt nämlich auch an, dass die jeweilige Stadt eine Universitätsstadt ist und damit ein gewisses Prestige verbinden will (man denke nur an die Ortsnamensschilder direkt an der Stadtgrenze: Universitätsstadt Tübingen!). Straßennamen sind also auch Träger einer Sekundärfunktion, durch die bestimmte, fast immer bewusst gesetzte, aber vom Rezipienten oft nicht bewusst erkannte Botschaften und Inhalte übermittelt werden sollen. Gottfried Korff spricht von „Notationssystemen, die eine starke bewußtseinsformierende, eine mentalitäts- und affektprägende Wirkung haben.“ (Korff 1992: 325). Man könnte auch davon sprechen, dass Hodonyme als Oberflächenindizes die Tiefenstrukturen einer Kultur abbilden, dass also die kulturellen Codizes einer Gesellschaft, Gemeinschaft, Gemeinde dem Sprachmaterial Straßenname regelrecht eingeschrieben sind. Die Perzeption offenbart Konzeption(en), und zwar die des Wahrnehmenden ebenso wie die hinter dem Wahrgenommenen stehenden Ideenentwürfe. Öffnet man den Blick auf diese Sekundärfunktion und Tiefenstruktur, so wird das Lesen eines Straßennamens zur kulturellen Nabelschau: Sage mir, wie du deine Straße, deine Städte, deine (Vor)-Orte, deine Stadien usw. benennst - und ich sage dir, wer du bist.

Insgesamt liefern uns Straßennamen mindestens vier Informationsebenen, die Bering und Großsteinbeck herausgearbeitet haben (Bering; Großsteinbeck 1999: 147-155):

1. Basisinformationen (nichtlinguistische Daten wie z.B. die Tatsache, dass sich in Köln das Straßenschild Heinestraße und die so benannte Straße im Stadtteil Lindenthal befinden);

2. Formebene (die morphologisch-syntaktische Struktur des Kompositums Beethovenstraße);

3. Bezugsebene (das konnotative Potential: Ludwig van Beethoven war Rheinländer, Komponist der Unvollendeten, er wurde taub etc.) sowie die

4. Bedeutungsebene (denotative Aspekte wie die Tatsache, dass Beethovenstraße ein Anthroponym ist, dass es eine kulturelle Funktion ausübt, einen lokalen, regionalen oder überregionalen Wirkungsbereich intendiert etc.).

Kurzum: Straßennamen sind Teil der städtischen Physiognomie und Teil des Erfahrungsschatzes eines Menschen bzw. einer Gruppe von Menschen, die mit der Benennung Beheimatungs-Erlebnisse verbinden. Viele Straßennamen sind aufgeladen mit „menmischer Energie“ (Assmann; Hölscher 1988: 12), indem sie neben ihrer erwähnten topografischen Funktion (Rheinuferstraße) Ankerpunkte in einem Wunschraum darstellen (Im Finkenhain, Pariser Passage) und oft auch 
Ausdruck einer gelenkten Erinnerungskultur sind (Tannenbergalle in Berlin, die an den Sieg der Schlacht bei Tannenberg gegen die russische Armee im Ersten Weltkrieg erinnern soll). Sie können Zeichen symbolischer Ortsbezogenheit sein und dabei als „verstecktes Politikum“ (Koß 1990: 91) wirken, dessen mnemische Potenz am augenfälligsten zutrage tritt, wenn bestimmte Interessensgruppen politisch-institutionellen Vorhaben der Umbenennung etablierter Straßennamen teils heftigen Widerstand entgegensetzen.

Die Straßennamenforschung lässt sich an drei wissenschaftlichen Horizonten verorten: Erstens erforscht sie stadtsemiotisch das Verhältnis von Topografie und Namenschatz, untersucht zweitens mentalitätsgeschichtlich die Straßennamengebung und -pflege als Ausdruck kollektiv unbewusster Sprachpraxis im Rahmen alltäglicher Wahrnehmungsweisen und versucht drittens, Straßennamen als identitätsstiftende und -sichernde, nicht selten politisch-administrativ gelenkte Bestandteile des „kulturellen Gedächtnisses“ (Assmann 1992) zu eruieren.

Bevor wir auf Gründe für eine Didaktisierung von Straßennamen in Gruppen von Deutschlernenden näher eingehen und konkrete Beispiele für Unterrichtseinheiten nennen, empfiehlt es sich zunächst, am Beispiel einer einzelnen Stadt einige grundsätzliche Aspekte von Benennungswandlungen aufzuzeigen. Es ist natürlich sinnvoll, sich vor dem eigentlichen Unterricht ein Grundwissen zu historisch-kulturgeschichtlichen Phänomenen der Straßennamen-Praxis anzueignen. Zentrale diachrone Aspekte sollen anhand der Stadt Köln kurz beleuchtet werden. Als Forschungsliteratur und gleichzeitig quellendokumentarische Fundgruben dienen uns in erster Linie die Ergebnisse der seit 1990 konstituierten Forschergruppe um Dietz Bering, wobei vor allem Peter Glasners zweibändige Dissertation zur Kulturgeschichte der mittelalterlichen Straßennamen Kölns (Glasner 2002) und Marion Werners umfassende Untersuchung für die Zeit von 1933 bis 1989 (Werner 2008) hervorzuheben sind.

\section{Historische Perspektiven}

\subsection{Vom Toponym zum Mnemotop: Straßennamenpraxis Kölns vom Mittelalter zur Moderne}

Vor der Wende vom 18. zum 19. Jahrhundert existierte noch kein breiteres öffentliches und schon gar kein wissenschaftliches Interesse an Straßennamen. Im Mittelalter waren sie Bestandteile einer weder administrativ reglementierten noch politisch gelenkten Sprachpraxis des Alltags, motiviert in erster Linie durch sinnliche Evidenz. In einer mittelalterlichen Stadt gab es weder Straßenschilder, noch waren die Straßennamen auf andere Weise optisch sichtbar. Ein der Stadt als Raum eingeschriebener symbolischer Zeichencharakter von Plätzen, Kirchen, Wohnhäusern und Verkehrsführungen konnte zuweilen ,unheimliche Konnotationen“ (Dinzelbacher 1993: 609) wachrufen. Da die mittelalterliche Kultur viel stärker als 
heute bestimmt war durch Religiosität, also durch die Gegenwart des Religiösen in der Lebenswelt, und durch Mündlichkeit, d.h. durch relative Schriftarmut und weit verbreitetes Analphabetentum, geschah die Aufladung mnemischer Energien vor allem mit Hilfe von religiös-liturgischen Ritualen, nicht aber vermittels des sichtbaren Sprachzeichens Straßenname. ${ }^{2}$

Am Beispiel des mittelalterlichen Kölns, damals eines der bedeutendsten urbanen Zentren Mitteleuropas, lassen sich vier Kernbereiche von Wahrnehmungsobjekten für die Straßennamen-Gebung herauskristallisieren: Erstens ökonomische Gesichtspunkte (Produktionsstätte, Handelsplätze), zweitens topografische Gegebenheiten (Flur- und Naturnamen), drittens religiöse Bezüge (Klöster, Kapellen, Heilige), viertens personenbezogene lokale Referenzen. Der vierte Bereich betrifft nicht-hageonymische Anthroponyma, also Personennamen nach städtischen Grundbesitzern und Familien, ferner nach stadtbekannten Außenseitern. Gerade die Personen- und Außenseiternamen eignen sich dazu, das exzeptionell Charakteristische an der mittelalterlichen Straßennamenpraxis herauszuarbeiten.

Mittelalterliche Anthroponyma-Straßennamen sind niemals aus einem Gestus des ehrenden Erinnerns entstanden, weswegen ihnen jede Vorbildfunktion fehlt. Alle Benennungen sind daraus motiviert, dass die entsprechenden Personen ursprünglich im Stadtraum ansässig waren (remers gaß, oirtmanns gaß). Da Benennungspraxis von Straßennamen durch dominante, phänomenologisch erkannte Fakten bedingt ist, ohne von Instanzen festgeschrieben oder kultiviert zu werden, kommt auch keinem einzigen der 198 Straßennamen auf dem Mercator-Plan von 1570/71 eine ideologische Bedeutung zu. Die Straßennamen auf diesem Plan verweisen auch nicht auf außerstädtisch oder gar reichsweit bekannte Persönlichkeiten. Ein Canossaplať, ein Beşanconplatz oder gar eine Barbarossastraß sind im mittelalterlichen Köln undenkbar. Dass Straßen und Plätze nicht nach Reichsfürsten, wohl aber nach Außenseitern benannt wurden, ist symptomatisch für den engen Zusammenhang von Sehen und Bezeichnen im Mittelalter. Fremde und Andere konnten deshalb namenmotivierend sein, weil sie - im Unterschied zum fernen Kaisers Friedrich Barbarossa - Bestandteil des alltäglichen Wahrnehmungsbildes waren: blynderjohansgasse; juedengaß; slungasse, benannt nach dort ansässigen Prostituierten (mhd. slun für „Hure").

Einer der zentralsten Gründe für mittelalterliche Straßennamen ist ihre topografische, ortsanzeigende Funktion. Sie haftet auch Straßennamen mit religiösem Bezug an (so liegt die apostel strai $\beta$ an der Apostelkirche, die S. Cicilien straiß an der Klosterkirche etc.), ist aber besonders augenfällig in den Straßenbezeichnungen dort ansässiger Zünfte, die innerstädtisch mitunter zu Namens-Kartografien des Handels vernetzt waren. So konnten die einzelnen Produktionsschritte des Wollgewerbes in der Kölner Altstadt nachverfolgt werden, und zwar genau in ergonomischer Reihenfolge: schaeffen straiß (Schafzucht), An der Wollkuchen (Woll-

2 Glasner hat, anhand einer akribischen Analyse der Quellen, diesen Zusammenhang am Beispiel Kölns in umfassenden Pionierstudien herausgearbeitet: Glasner 2001; 2002a; 2002b. 
waschen), keimer gaß (Wollkämmen), Weber straiß (Weben), foller straiß (Walken), schaers gaß (Wollscheren), onder glaferber (Färben).

Während Bering und Großsteinbeck (1994) eine nahezu identische Relation von Benennung und Realität noch für das spätmittelalterliche Köln hervorheben, ${ }^{3}$ stehen für Glasner (2001) Stadt und Namenschatz trotz der genannten Wahrnehmungsmotivierung da schon nicht mehr in einem spiegelartigen Abbildverhältnis zueinander. ${ }^{4}$ Vielmehr zeige die mittelalterliche Nominationspraxis ein eigenes, codiertes Bild aus Betonungen und Ausblendungen, das seine mentalitätsgeschichtlichen Brechungen in der Stadtwahrnehmung bereits ansatzweise enthülle und deshalb als „Mentalitätsgeschichte des Sehens“ zu bezeichnen sei (Glasner 2002a: 45). Hierbei kommen drei zentrale methodische Prinzipien zum Tragen:

1. Prinzip der (sinnlichen) Evidenz: Straßennamen sind symptomatisches, eben evidentes Zeichen eines Erscheinungsbildes der Namensträger; der Zusammenhang von Bezeichnetem und Bezeichnendem im Moment der Übereinstimmung ist auf ein charakteristisches Merkmal hin verdichtet: blynderjohansgasse

2. Prinzip der Relevanz: Die zeitspezifische Bedeutung eines StraßenMerkmals - also der „mentalitätsgeschichtliche Zeitbezug“ (Peter Glasner) entscheidet über dessen Benennung; die Namensgebung ist gleichbedeutend mit einem Code, der Wahrnehmungsschwerpunkte setzt (Bsp: Teilstück der heutigen Hohe Straße $\rightarrow$ 1260: Lapidea platea nach der damals seltenen Pflasterung; 1449: under spermecheren nach den dort ansässigen Speermachern)

3. Prinzip der Varianz/Konsensorientierung: Jeder Namens-Entscheidung gehen insofern unbewusste Auswahlprozesse voraus. Über Varianzen kollektiver Wahrnehmungen gelangen die Stadtbewohner zu einem Konsens, der bei jeweils veränderten Wahrnehmungspräferenzen wiederum korrigiert werden kann.

Die mittelalterliche Praxis, Straßennamen in erster Linie als Abbildung städtischer Gegebenheiten und als Bilder einer Geschichte des Sehens zu nutzen, änderte sich in Köln erst allmählich im 18. Jahrhundert, ganz entscheidend dann mit der Besetzung der Stadt durch die Franzosen Anfang des 19. Jahrhunderts. Der reichsstädtische Universitätsrektor Ferdinand Franz Wallraf, von den französischen Besatzern 1812/1813 mit der Übersetzung der da noch unsystematischen und nicht verschriftlichten Straßennamen beauftragt, charakterisiert die größtenteils aus dem Mittelalter herrührenden Benennungen mit wenig schmeichelhaften Worten:

\footnotetext{
${ }^{3}$ Bering; Großsteinbeck 1994: 106: „Die Namen auf dem Mercator-Plan sind im großen und ganzen nichts anderes als der Spiegel der Wirklichkeit in geographischer, sozialer und wirtschaftlicher Hinsicht."

${ }^{4}$ Glasner 2002b: 41: „Das Verhältnis der Stadt zu ihrem Namensschatz ist also kein spiegelartiges Abbildungsverhältnis, sondern ein eigenes Bild aus Betonungen und Ausblendungen.“
} 
„An den mehrsten unserer bisherigen Straßen und Gassen kleben wirklich nur pöbelhafte, seichte, unsichere, ihrer Herleitung nach oft so unbedeutende, größtenteils in den Zeiten der crassesten Ignoranz entstandene und nur durch Gleichgültigkeit und Gewohnheit angenommene [...] Benennungen [...], deren einiger wir uns vor allen fremden Ohren schämen müssen." (Zit. n. Kramer 1993: 230).

Wallraf startete ein Projekt, das den Stadtraum wie ein Erinnerungsbuch ausstaffieren sollte. Geleitet von aufklärerischem Eifer und klassizistischen Idealen sollten die Kölner Straßennamen, seit 1812 sukzessive an Häuserwände gemalt, nun einen vermeintlich ehrwürdigeren Charakter bekommen. Pissgasse, Schmierstraße oder Hunderücken wurden in Börsengasse (Passage de la Bourse), Komödienstraße (Rue de la Comédie) und Hunnenrücken umbenannt. Durch Straßennamen wie Trajanstraße, Capitolstraße und Agrippa-Platz sollte die "glorreiche“ Antike symbolisch wieder ans kollektive Gedächtnis gekoppelt und zugleich die Erinnerung an das als dunkel empfundene Mittelalter überwunden werden.

Nicht die alltägliche Wahrnehmung wie im Mittelalter (in Spilmannsgassin, mördergasse), sondern Wunschbilder und identitätssichernde Erinnerung standen im Vordergrund dieses neuen Programms, das mit der Preußenherrschaft über Köln 1880 bis 1890 einen weiteren Höhepunkt erreichte. Die stilisierte Kartografie einer deutschen Geschichte am Rhein, in präziser Reihenfolge beginnend am Bayenturm in der Südstadt und kulminierend in den Deutschen Ring, findet in dieser Tendenz zur Ideologisierung der Straßennamen ihre Begründung: Ubierring und Chlodwigplatz. sollten an die germanischen Ursprünge gemahnen, Karolinger Ring, Salierring, Barbarossaplatz und Hohenstaufenring an königliche Herrschergeschlechter des Mittelalters erinnern, der Habsburger Ring an die Stabilisierungs- und Endphase des Reichs unter der österreichischen Dynastie, der Hohenzollernring und der KaiserWilhelm-Ring an die preußisch dominierte Neuinstallierung der Reichseinheit denken lassen. Ein längerer Spaziergang entlang dieser Ringe konnte so schnell zum historischen Erinnerungs-Parcours geraten.

Seit Anfang des 19. Jahrhunderts fungierten die Straßennamen nicht mehr als Topografien oder als Realitätsbenennungen entlang der Wahrnehmungsweisen des kommunikativen Alltagsgedächtnisses, sondern als Mnemotope, als Denkmäler mit Belehrungsfunktion. Von daher wird auch verständlich, weshalb neuzeitliche Straßen - oft in Cluster-Bildung - nach (zumeist verstorbenen) militärischen, kulturellen, religiösen oder politischen Leitfiguren, aber in aller Regel nicht - wie im Mittelalter - auch nach Randgruppen oder Außenseitern benannt wurden: Der Zusammenhang von Wahrnehmen und Bezeichnen hatte einer Erinnerungskultur zu weichen, die, da sie gelenkt war, viel konsequenter das eine aufnahm, das andere eskamotierte.

Freilich barg und birgt dieser politisch installierte und nicht genuin aus der Alltagspraxis der Bewohner stammende Prozess der Historisierung, Ideologisierung, Ästhetisierung und Kultivierung des semiotischen Systems Stadt mittels Straßen- 
namen ein nicht unbeträchtliches Problematisierungspotenzial: Einmal im identitätskonkreten kollektiven Gedächtnis eingeschriebene und in der Form des Sprachzeichens Straßennamen verdichtete Erinnerungen können zumeist nicht widerstandslos ad hoc durch andere Wissensbestände ersetzt werden. „Soziales Erinnern heißt immer auch öffentliches Ringen um elementare Bausteine des kollektiven Selbstverständnisses.“ - Diese Analyse Glasners (Glasner 2002b: 20) trifft auf diachroner Ebene auf viele historische Dissense um Straßen(um)benennungen zu. Sie gilt für den Konflikt, der am 6. Juli 1922 im Kölner Rat in einem

Tumult endete, als die SPD-Fraktion vorschlug, aus Anlass der Ermordung des Außenministers Walter Rathenau den Kaiser-Wilhelm-Ring in Walter-Rathenau-Ring umzutaufen und ein polemischer Zwischenruf die Gemüter erhitzte; und sie gilt sechzig bzw. siebzig Jahre später auch noch für die langwierige Auseinandersetzung um die Frage, ob der Reichenspergerplatz oder der Appellhofplatz zu einem Heinrich-Böll-Platz werden dürfe oder ob der an Schlesien erinnernde Breslauer Platz gegen das weltpolitische Schwergewicht Willy Brandt ankommen könne.

In diesen Ausführungen wurden noch einmal einige der wichtigsten Prinzipien zusammengefasst, nach denen neuzeitliche Straßennamen-Gebung funktioniert. ${ }^{5}$ Sie begleiten den seit der Franzosenherrschaft einsetzenden Paradigmenwechsel urbaner Gestaltungspräferenzen vom alltäglichen Abbild zum unverwechselbaren Denkbild und sind deshalb abschließend eigens zu explizieren:

1. Prinzipien der Raumgliederung und Richtungsweisung: Die Zusammenfassung von Straßen zu Straßenvierteln durch spezifische Gattungsnamen erleichtert das Zurechtfinden. Cluster-Bildungen helfen, Räume besser erfahrbar zu machen: Markomannenstraße, Alemannenstraße, Teutonenstraße; daneben bleibt die richtungweisende Orientierungsfunktion von Straßen mit Städtenamen in aller Regel gewahrt: Aachener Straße, Neusser Straße.

2. Prinzipien der Eindeutigkeit und Gleichwertigkeit: Straßennamen müssen unverwechselbar sein, weswegen Namendopplungen auszuschließen sind; die Bedeutsamkeit des Namensträgers muss in etwa mit der Bedeutung der Verortung des Straßennamen korrespondieren.

3. Prinzip der (nationalen, regionalen, lokalen oder ideologischen) Identitätsstiftung bzw. - sicherung: Auf Straßennamen werden gedächtniskulturelle Inhalte projiziert, die mittels konkreter lokaler oder regionaler Bezüge der seelischen Beheimatung der Stadtbewohner dienen bzw. nationale Erinnerungswerte festhalten sollen; sie können auch ganz im Dienst einzelner Ideologien stehen.

4. Prinzip der Heilung/Verschleierung lebensweltlicher Diskrepanzen: Mittels euphemistischer (z.B. idyllischer) Beheimatungsnamen in Reißbrettvierteln soll die gedächtniskulturelle Armut eines eben noch nicht „gewachsenen“ Viertels ausgeglichen werden (ein Beispiel ist die 1921 erfolgte Straßen-

5 Vgl. zu diesen und weiteren „Gesetzen“ bzw. Prinzipien für moderne Namensgebung: Werner 2008: 232-241. 
namen-Vergabe in der Neusiedlung Bickendorf nach Naturnamen: Unter Birken, Unter Bergamotten).

Teilweise gelten diese Prinzipien bis in die heutige Zeit, in der sich im ohnehin immer unruhiger werdenden Zeichenfeld Stadt die Denkbilder rasant verändern. $\mathrm{Ob}$ man angesichts einer Kölner Toyota-Allee (1995) schon vom beginnenden Ausverkauf der Erinnerungskultur oder, weniger dramatisch, von ersten Schritten zu einer Neuformulierung der Wissensbestände im Sprachzeichen Straßennamen sprechen kann, ist eine von vielen Fragen, die sich dabei aufdrängen.

\subsection{Straßennamen als historische Quellen}

Hodonyme sind im Stadtraum sichtbare, alltägliche Zeichen und Sachquellen, aber an historischem Quellenwert gewinnen sie erst, wenn über sie in dokumentierbarer Weise reflektiert, diskutiert, entschieden wird, wenn also ein Diskurs entsteht, der sich wiederum in Alltagszeugnissen wie Leserbriefen, Zeitungsartikeln, Ratssitzungsprotokollen, administrativen Dokumenten etc. widerspiegelt. Vor allem das diskursiv-kommunikative Für und Wider bei erfolgreichen oder gescheiterten (Um-) Benennungen von Straßennamen macht sie zu einer historischen Quelle, die sich für Didaktisierungen eignet: „Gerade weil sich Kulturen als historisch gewachsene Resultate von Kommunikationsprozessen darstellen, sollte ihre Beschreibung sinnvoller Weise auch an konkreten Kommunikationsprodukten orientiert sein, wobei natürlich insbesondere solche Quellen aufschlussreich sind, die derartige Kommunikationsprozesse thematisieren." (Bolten 2007: 26).

Es war bereits erwähnt worden, dass die Straßennamen Kölns als historische Quellen für den Unterricht auch deshalb so prädestiniert sind, weil erstens zu ihnen eine umfängliche Forschungsliteratur existiert, auf die der Lehrende zurückgreifen kann, kurzum: Das theoretische Fundament ist gesetzt. Zweitens verfügt die Stadt Köln mit ihrer über 2000jährigen Geschichte nicht nur über ein seit 1130 fast lückenlos dokumentiertes Schreinswesen (den Vorläufern des heutigen Grundbuches), sondern auch über ein Zentrales Archiv für Straßenbenennungen - insgesamt also über ein Korpus an Primärquellen und Forschungsliteratur, das unter den deutschen Großstädten seinesgleichen sucht. Darüber hinaus existiert zu Köln eine Vielzahl von historischen Stadtführern, die - meist in anekdotischer Erzählweise - auch Hintergründe zu Kölner Straßennamen liefern. ${ }^{6}$ Folgende Quellen sind für die Straßennamensforschung in Köln besonders relevant:

${ }^{6}$ Z.B. Kaufman; Lutz; Schmidt-Esters 1996; Jung 2004; Klever 2001; Schmid 2005; Priebe 2008. Natürlich ließe sich eine solche Liste an kulturell orientierten Reiseführern anhand von Straßennamen und Plätzen auch problemlos für andere Großstädte finden, wie z.B. Berlin. 


\section{a) Mittelalter}

1. Schreinsbücher (Vorläufer des Grundbuches, zunächst in Latein, ab dem 13 Jh. aber auch verstärkt in Deutsch: 1130-1789) mit 150000 Eintragungen allein bis zum Jahr 1400;

2. Erinnerungsbücher des Ratsherren Hermann von Weinsberg (15181597), in denen Weinsberg sich sogar an etymologische Deutungen von Straßennamen versucht;

3. Stadtplan von Arnold Mercator (1570/71) mit 196 Örtlichkeitsangaben: Eine um Entsprechung bemühte, wissenschaftlich-kartografische Darstellung Kölns aus der Vogelperspektive, im Unterschied zu den typisiert-idealisierten Stadtbildern in Tafelmalereien, Holzschnitten und Kupferstichen des 16. Jahrhunderts.

\section{b) Nenzeit}

1. Adressbücher: ab 1795 (inkl. kurzer etymologisch-historischer Deutungen);

2. Zentrales Straßennamenarchiv (in Köln für jede Straße eine Akte: über 5000 Einzelakten!);

3. Artikel in den lokalen Medien, vor allem in Tageszeitungen wie „Kölner Stadt-Anzeiger“, „Kölnische Rundschau“, „Express“), aber auch in Rundfunk und Lokalfernsehen (WDR etc.)

Besonders hilfreich für Unterrichtseinheiten zur Straßennamen-Praxis seit der Neuzeit sind gerade alle online und kostenfrei zugänglichen Artikel der lokalen Tagespresse.

\section{Didaktische Perspektiven}

\subsection{Gründe für die Behandlung von Straßennamen im DaF-Unterricht}

Eine Didaktisierung von Straßenschildern in Gruppen von Deutschlernerinnen und -lernern erscheint besonders aus folgenden Gründen sinnvoll:

1. Dass sich jede Sprache auf diachroner Ebene wandelt, ist oft beschrieben worden (z.B. bei Keller 1994). Sprachliche Zeichen sind arbiträr und historisch konventionalisiert. Variantenreichtum ist dabei ein grundlegendes Merkmal sprachlicher Realisation. Dies gilt auf grammatikalischer wie lexematischer Ebene. Was aber im Bereich der Grammatik bei Deutschlernerinnen und -lernern zu Verunsicherung führen könnte - schließlich tendieren Fremdsprachenlerner vor allem im Anfängerbereich dazu, die Sprache erst einmal als feststehendes System begreifen zu wollen und nicht als ein stetem Wandel unterworfenes Phänomen stellt im Bereich der Straßennamen eine Bereicherung dar. DaF-Lerner erfahren, 
dass es ganz bestimmte historische, politische, kultur- und mentalitätsgeschichtliche Gründe für die Bennennung und Umbenennung von Straßen gibt. Die Lerner/-innen können somit das wichtige Phänomen des Sprachwandels konkret erfassen, ohne sich in schwierige und abstrakte linguistische Theorien einarbeiten zu müssen.

2. Straßennamen eignen sich besonders gut, den Lernenden die immense Bedeutung von Signifikanten in einem kulturell-politischen Kontext zu vermitteln. Sie zeigen, welch starke mnemische Energie die Konnotate von Denotaten entfalten können. Der sprachliche Vollzug ist immer auch Ausdruck einer bestimmten „Weltansicht" (Humboldt 1830-35: 60), und zwar sowohl auf individueller wie auf kollektiv-nationaler Ebene. Namen sind nun besonders eng an Identiät gebunden, können diese fördern, aber auch gefährden. Straßennamen schildern dabei zweierlei: Erstens weisen sie auf synchroner, aber vor allem auch diachroner Ebene Wege zum kollektiven Gedächtnis einer Stadt; zweitens demonstrieren sie, dass Sprache immer auch ein Politikum sein kann. Jung fasst dies folgendermaßen zusammen: „Wer als Deutschlernender mehr erfahren möchte als die Abläufe der Deklination von Substantiven oder der Konjugation von Verben, der hat mit dem Straßenschild ein Sesam-öffne-dich zur Hand, das ihm hilft, die Tür zum Deutschtum einen Spalt zu öffnen." (Jung 2005: 95). ${ }^{7}$

3. Die Praxisrelevanz von Straßennamen fällt schnell ins Auge. Als „lebendiges Sprachmaterial“ (Schultheis; Walter 1968: 7) bieten sie den unschätzbaren Vorteil lebensweltlicher Alltagsnähe, denn sie sind Teil dessen, was Jan Assmann im Rückgriff auf die Theorien von Maurice Halbwachs das „kommunikative Alltagsgedächtnis“ genannt hat (Assmann 1992: 50): Jeder kennt Straßennamen, so gut wie jeder wohnt an einer Straße, die einen Namen trägt und verbindet damit bestimmte Erinnerungen. Sie erfüllen also in besonderer Weise zentrale Forderungen einer handlungsbezogenen Sprachdidaktik: Handlungsorientierung und Nutzbarkeit der Sprache unter Berücksichtigung verschiedener Ebenen der Sprachverwendung (sozial, politisch, pragmatisch etc.); Vorrang sprachlicher Funktionen vor rein formalen Aspekten; Einsatz authentischer Texte, Quellen, Übungen (vgl. Roche 2005: 215f.).

4. Sie bieten darüber hinaus besonders vielfältige Möglichkeiten zur Erfahrbarkeit, Visualisierung und Medialisierung. Straßennamen haben immer auch einen alltäglichen, ganz praktischen Gebrauchswert. DaF-Lerner können erkennen, dass Kenntnisse beispielsweise über die Cluster-Bildung von Straßennamen (also die areale Konzentrierung von Straßennamen nach Bennennungsmotiven wie Komponisten, Feldherren, Tiernamen etc.) ihnen konkret helfen können, sich in einer ihnen zunächst fremden deutschen Stadt besser zu orientieren. Mittels Internet und Straßenplänen wird die Suche nach dem Gedächtnis der Stadt visualisierbar, mittels angeleiteter Parcours-Erkundungen erlebbar.

\footnotetext{
7 Zit. auch bei Oebel 2006, 571. Es versteht sich von selbst, dass die Verwendung von problematischen, weil historisch-ideologisch belasteten Begriffen wie „Deutschtum“ im DaFUnterricht kritisch reflektiert werden muss.
} 


\subsection{Didaktische Literatur zum Thema}

Entscheidende Hinweise darauf, dass die doppelte Funktion von Straßennamen die topographische Orientierungs- und die kulturelle Verweisfunktion, also ihr Gebrauchswert plus Kulturwert - den Einsatz von Hodonymen im Fremdsprachenund Landeskundeunterricht sinnvoll macht, finden sich recht früh bei Udo Jung (Jung 2000: 609f; Jung 2005). Er konstatiert: „In ihrer Summe stellen die Straßennamen nämlich das Gedächtnisbuch einer Stadt dar, und eine Klasse von (Fremd-) Sprachenlernern kann darin blättern und recherchieren, die Ergebnisse mit denen anderer Städte vergleichen und sie zueinander in Beziehung setzen.“ (Jung 2000: 610).

Mein Aufsatz verdankt zentrale Anregungen einer Studie von Guido Oebel aus dem Jahre 2006, in der die Relevanz und die geradezu unerschöpflichen Einsatzmöglichkeiten des Themas Straßennamen im DaF-Unterricht aufgezeigt werden (Oebel 2006: 569-583). Einen Reader zur Namenskunde mit Aufsätzen zur Reflexion des Themas in der Primarstufe und in den Sekundarstufen I/II haben Frank und Koß bereits 1994 angelegt; indes geht darin noch keiner der darin enthaltenden Aufsätze explizit auf das Thema Straßennamen ein (Frank; Koß 1994). Selbst bei neuesten Internetportalen, die umfangreiche Unterrichtsmaterialen zu Namen zur Verfügung stellen, fehlen Hodonyme weitgehend bzw. lassen die spezifischen Anforderungen an den DaF-Unterricht unberücksichtigt. ${ }^{8}$

\subsection{Eingrenzung der Lernerzielgruppe und des Themas}

Das Thema eignet sich sowohl für weniger fortgeschrittene als auch für fortgeschrittene bzw. weit fortgeschrittene Deutschlernende. Im DaF-Unterricht mit Deutsch als dominierender Unterrichtssprache sollte aber ein Sprachniveau auf dem Level von ca. A2 (entsprechend CEFR) oder höher vorausgesetzt sein, da vorher (A1) oder im reinen Anfängerbereich noch die entsprechenden Vokabelund Grammatik-Kenntnisse fehlen, um sich über Prozesse, Debatten und Hintergründe zu Straßenbenennungen und -umbenennungen sinnvoll austauschen zu können. Das Thema eignet sich besonders gut im Landeskundeunterricht, aber auch im Bereich des interkulturellen Lernens, wobei Fragen im Vordergrund stehen können wie: Warum sind in Deutschland in jedem noch so kleinen Dorf Straßen und Plätze nahezu vollständig benannt, während es in einer so gewaltigen Metropole wie Tokyo mit seinen fast neun Millionen Einwohnern im Kerngebiet keine stringente Straßenbeschilderung gibt?

Im Prinzip könnte man die Straßennamen-Thematik auch für orthografische und sogar grammatische, z.B. flexionsspezifische Fragestellungen nutzen. Der Rechtschreib-Duden (2006) widmet der Rechtschreibung von Namen und der Setzung der Bindestriche immerhin ganze sechs Seiten (Duden 2006: 86-91, K

8 Z.B. bei: http://www.lehrerfreund.de/in/schule/1s/namen-unterrichtsmaterial-arbeitsblaetter/ („Namen/Namenskunde im Deutschunterricht - Unterrichtsmaterialien“). 
134-151), denen sich auch ein eigenes Kapitel zu Straßennamen anschließt (ebd.96f., K 161-163). So ließe sich etwa fragen, wieso bei bestimmten Straßennamen Bindestriche gesetzt werden (Heinrich-Mann-Alle), bei anderen aber nicht (Adenauer Ufer), ob dies willkürlich geschieht oder ob hier eindeutigere Regelhaftigkeiten herrschen als die im Duden (Duden 2006: 87, K 136) angegebenen: „Einen Bindestrich kann man setzen, wenn der Name hervorgehoben werden soll oder wenn dem Namen ein zusammengesetztes Grundwort folgt". Auch national- bzw. regionalspezifische Varietäten des Deutschen könnten im Fokus des Unterrichts stehen, beispielsweise die im Gegensatz zur deutschen Praxis stehende schweizerische Sonderregelung, dass „Straßennamen, die die Ableitung eines geografischen Namens auf -er enthalten, gewöhnlich zusammengeschrieben [werden] (Hottingerplat, Winterthurerstraße).“ (Rechtschreib-Duden 2006: 96, Zusatz zu K 162). Weiterhin könnte das Problem der Fugenelemente im Deutschen zum Thema werden, beispielsweise das Phänomen des so genannten Fugen-s: Wieso heißt es Reichstagsufer und nicht, was als Kompositum eigentlich logischer erscheint, Reichstagufer?

Solche rein orthografisch-grammatischen Schwerpunktsetzungen erschöpfen sich aber schnell thematisch und verspielen eher die Chance, im Unterricht die reine Sprachebene mit der Kulturebene zu verbinden. Interessanter erscheint hier die Frage nach kulturell-sozial motivierten Regeln für das letzte Kompositum bei Straßennamen, beispielsweise im Hinblick auf Diminutive: Nach welchen außersprachlichen Kriterien wird entschieden, dass die Straße ein Wilhelm-Busch-Platz und kein -Plätzchen ist? Nach dem vorgeblichen Bedeutungsgrad des ersten Namensbestandteils? Nach dem ästhetisch-atmosphärischen Wert des Ortes? Nach dem semantischen Wert des Namensgebers? Nach den politischen Machtverhältnissen in der Stadt?

\subsection{Unterrichtseinstiege}

In den nächsten Kapiteln sind Vorschläge für Unterrichtseinstiege und Unterrichtseinheiten zu sehen. Als Beispiele für weiterführende Unterrichtseinheiten werden wiederum Straßennamen der Stadt Köln ausgewählt. Im Prinzip ließen sich die Methoden jedoch auf jede deutsche Stadt anwenden. Als Unterrichtseinstiege biete ich einmal einen klassischen Frage-Antwort-Dialog in Form eines Brainstormings und zweitens visuelle Diskussionsanreize an.

\subsubsection{Brainstorming mittels geleiteter Fragen}

In einem ersten Verfahren könnten den Lernenden folgende Fragen präsentiert werden, auf die dann induktiv-assoziativ zu antworten wäre: 
Tabelle 19

Mögliche Fragen als Unterrichtseinstieg in das Thema Straßennamen

1. Welche deutschen, österreichischen, schweizerischen Städte kennen Sie?

2. Waren Sie schon einmal in einer dieser Städte zu Besuch?

$\rightarrow$ Wenn ja: Wie haben Sie sich dort orientiert?

$\rightarrow$ Wenn nein: Wie orientieren Sie sich in einer Stadt, die Ihnen unbekannt ist?

Falls „Straßenschilder“/,Straßennamen“ angesprochen werden, ließe sich weiter fragen:

3. Können Sie sich an bestimmte Straßennamen erinnern?

$\rightarrow$ Wenn ja: An welche?

$\rightarrow$ Wenn nein: Haben Sie schon einmal von folgenden Straßennamen gehört: Kö(nigsallee), Alex(anderplatr), Ku(rfürstendamm)? Was fällt Ihnen auf?

4. Gibt es Straßennamen, die in verschiedenen Städten identisch sind? [interkulturelle Ebene]

5. Gibt es Unterschiede zwischen Straßennamen aus Ihrem Herkunftsland und Straßennamen in Deutschland, Österreich, der Schweiz, Belgien und Luxemburg (deutschsprachige Teile)? [interkulturelle Ebene]

6. Wer entscheidet Ihrer Meinung nach über die Benennung von Straßen (auch: von Plätzen, Sportstadien etc.)? [politisch-soziale Ebene]

7. Straßenschilder ändern sich oftmals über die Jahre und Jahrzehnte hinweg. Welche Gründe kann es für Umbenennungen geben?

$\rightarrow$ Falls dies nicht ergiebig sein sollte, könnte z. B. folgende Anregung weiterhelfen:

In Köln hatte ein und derselbe Platz über die Jahre hinweg die folgenden Straßennamen, und zwar in dieser Reihenfolge: Platz der Republik, Adolf-HitlerPlatr, Ebertplatz: Können Sie sich Gründe dafür denken, weshalb derselbe Ort so unterschiedlich benannt wurde? [historische/kulturell-mentalitätsgeschichtliche Ebene]

8. Was kann man anhand von Straßennamen über die deutsche Sprachgeschichte/Mentalität/Kultur erfahren? [historische/kulturell-mentalitätsgeschichtliche Ebene]

Die Fragen 1 bis 5 eignen sich auch für weniger fortgeschrittene Deutschlernende (ab A2), während die Fragen 6 bis 8 ein höheres Sprachniveau (ab B1) voraus-

${ }_{9}^{9}$ Tab.1 basiert teilweise auf dem Fragenkatalog bei Oebel 2006: 571. 
setzen und als Einstiege in längere Unterrichtseinheiten mit möglichen Hausaufgaben dienen können.

\subsubsection{Visualisierung mittels Fotografien}

Alternativ böte sich als Unterrichtseinstieg an, den Deutschlernenden bestimmte Fotografien anzubieten und darüber diskutieren $\mathrm{zu}$ lassen. Immer kritisch mitberücksichtigen muss der Lehrende die oftmals manipulatorische Wirkung von Bildern, wobei Fotografien von Straßennamen weniger Gefahr laufen, zu monoperspektivischem Geschichtsdenken zu verleiten, da aktuelle Abbildungen von Straßenplänen oder Straßenschildern ohne zusätzliche Textinformationen oder wertende Textkommentare erst einmal weitgehend fixierte und wertfreie Dokumentationen städtischer Gegebenheiten sind. Die folgende Auswahl versteht sich zudem ausschließlich als Anregung.

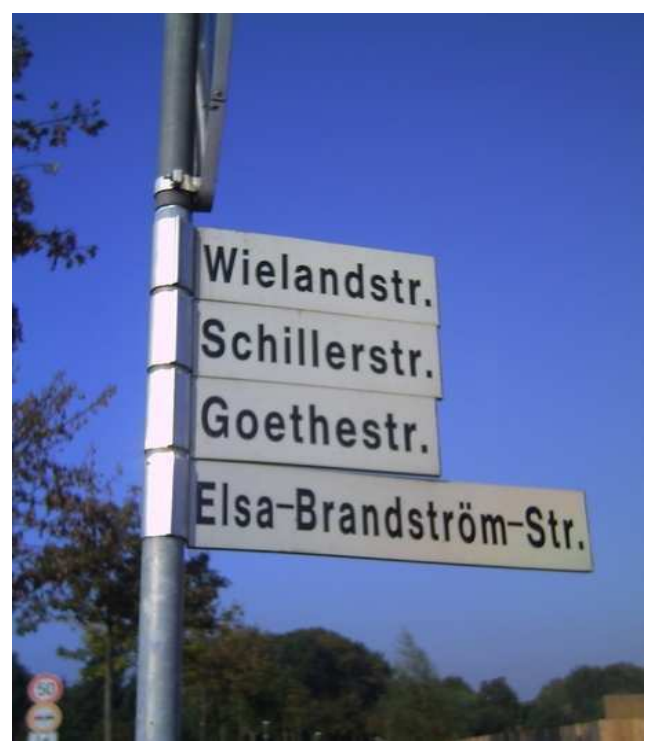

Abb.1: Dichterstraßen.

Quelle: http://data.lustich.de/bilder/1/13189-strassennamen.jpg.

Arbeitsanregungen:

Stellen Sie sich vor, Sie sind das erste Mal in einer deutschen Großstadt, versuchen sich zu orientieren und finden die im Bild gezeigten Schilder. Was würden Sie denken? Was fällt Ihnen an diesem Bild auf? Welche Namen werden genannt? 
Machen Sie Vorschläge, wie ein ausländischer Besucher einer Stadt mit diesem Straßenschild ein paar wichtige Hintergrundinformationen zu den Namensträgern erhalten könnte.

Halten Sie eine kurze Präsentation von 10 Minuten zu den Namensträgern der Wielandstraße und der Elsa-Brandström-Straße. Wer waren diese beiden Persönlichkeiten, was hat sie ausgezeichnet?

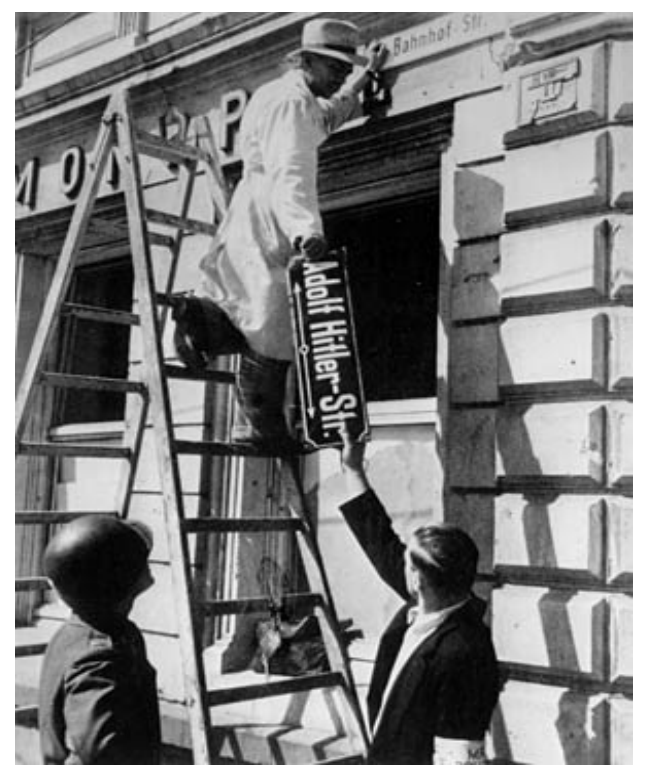

Abb.2: Adolf Hitler-Straße.

Quelle: http://www.history.army.mil/books/wwii/Occ-GY/ch15.htm.

Arbeitsanregungen:

Was wird auf der Fotografie dargestellt? Schauen Sie sich die drei abgebildeten Personen genau an. In welcher Zeit ist das Foto wohl aufgenommen worden?

1933 wurde in der Stadt Köln der Platz der Republik in Adolf-Hitler-Platz. umbenannt. Finden Sie Gründe für diese Umbenennung, indem Sie den zeitgeschichtlichen Hintergrund kurz skizzieren. Wie heißt der Platz heute? 


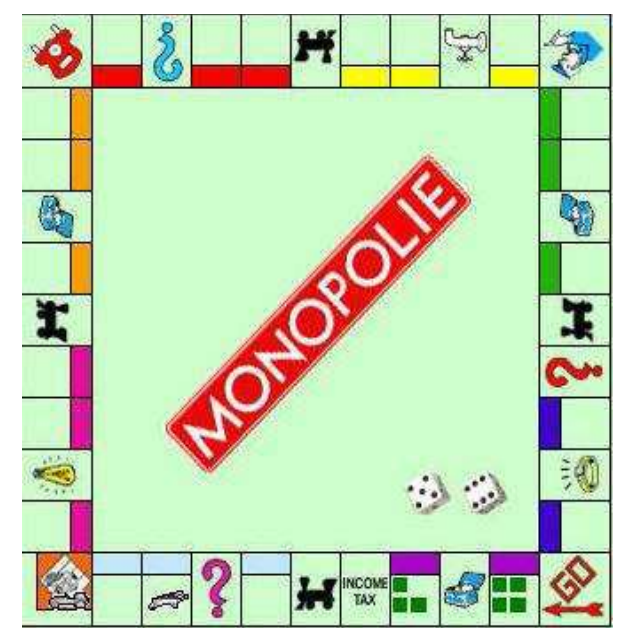

\section{Abb.3: MonopoliH}

Quelle: http://monopolie.sourceforge.net/screenshots.shtml.

Arbeitsanregung 1:

Dies ist eine englische Version des beliebten Brettspiels „Monopoly“. Ziel des Spiels ist es, ein Grundstücksimperium aufzubauen und die Mitspieler in den Konkurs zu treiben. Die Grundversion hat 22 Straßen, vier Bahnhöfe und je ein Elektrizitäts- bzw. Wasserwerk. Je nach Straße und Straßenviertel steigt oder sinkt das Mitpreisniveau. Die Straßennamen fehlen auf der obigen Abbildung. Im Folgenden finden Sie eine Liste mit Straßennamen, denen Sie Mietpreise von 60 bis 1000 Euro zuordnen sollen.

Ordnen Sie die Straßennamen Mietpreisen zu und tragen Sie beides anschließend in das Spielfeld ein, z.B. Glücksallee $1000 €$, gelbes Feld oben rechts. Schlagen Sie die Bedeutungen von (Straßen-)Namen nach, wenn Sie diese nicht kennen.

Straßennamen:

Lessingstraße; Operplatz; Mördergasse; Nordbahnhof; Glücksallee; Schillerstraße; Turmstraße; Badstraße; Goethestraße; Elisenstraße; Pechstraße; Beethovenstraße; Schlossallee; Hinterhofstraße; Hafenstraße; Mozartstraße; Babnhofstraße; Parkstraße; Arbeitslosengasse; Bachstraße; Verliererstraße; Chausseestraße

Mietpreise:

von $60 €$ bis $1000 €$

Arbeitsanregung 2: 
Begründen Sie Ihre Entscheidung! Fällt Ihnen ein Muster auf, nach dem Sie die Straßennamen eingeordnet haben? Warum haben Sie einer bestimmten Straße ein höheres Mitpreisniveau gegeben als einer anderen?

Einige der Namen in der Tabelle stammen tatsächlich aus der deutschen Version von „Monopoly“, andere sind frei erfunden. Welche sind das wohl?

Welche Straßennamen existieren in deutschen Städten so nicht (mehr) warum wohl nicht?

\subsection{Weiterführende Unterrichtseinheiten}

Im Folgenden sollen einige weiterführende Unterrichtseinheiten dargestellt werden, die auf die aufgezeigten Unterrichtseinstiege aufbauen können.

\subsubsection{Einordnung von Straßennamen nach Motiven der Benennung}

Es war schon angesprochen worden, dass in vielen Städten Straßennamen oft in so genannten arealen Clustern angeordnet sind, die einem bestimmten Benennungsmotiv folgen: Dichterviertel, belgisches Viertel, Industrieviertel, Obstsorten etc. Diese Tatsache lässt sich sinnvoll für den DaF-Unterricht nutzen. Die Lernenden werden in Arbeitsgruppen aufgeteilt und erhalten eine Reihe von Straßennamen, die sie nun bestimmten Motiven zuordnen müssen (vgl. Oebel 2006, 572f.). Dazu empfiehlt es sich natürlich, den Lernenden die Möglichkeit zur Recherche zu geben (Internet, Bibliothek), also die Lerneinheit mit einer Hausaufgabe zu verknüpfen.

Tabelle 2

Sektion I: Anthroponyme (Personen- und Familiennamen) und Phytonyme (Tier- und Pflanzennamen)

Gruppe A: Schriftsteller

Heinrich-Böll-Platr, Goethestraße, Schillerstraße, Lessingstraße, Hoffmann-vonFallersleben-Straße, Gorch-Fock-Straße, Oscar-Wilde-Straße, Ricarda-Huch-Straße, Rilkestraße, Heinestraße, Rahel-V arnhagen-Straße, Irmgard-Keun-Straße etc.

Gruppe B: Maler und Musiker

Brabms-Straße, Lovis-Corinth-Straße, Spitzweg-Straße, van-Gogh-Straße, JacquesOffenbach-Platz, Beethovenpark, Richard-Wagner-Straße, Mozartstraße etc.

Gruppe C: Philosophen und Wissenschaftler

Nietzschestraße, Schlegelstraße, Kantstraße, Feuerbachstraße, Karl-Marx-Allee, Friedrich-Engels-Straße etc.

Gruppe D: Heilige und Kirchenvertreter (Hageonyme)

Georgstraße, Severinstraße, Christophstraße, Ursulastraße, Martin-Luther-Platr, Bonhoefferstraße, Prälat-Otto-Müller-Platr, Präses-Richter-Platr, Pastor-Paul- 
Milde-Straße etc.

Gruppe E: Politiker, Funktionäre, Unternehmer

Bebelplatr, Clara-Zetkin-Straße, Cornelius-Stüssgen-Straße, Friedrich-Ebert-Plat, Robert-Schumann-Straße, Kennedy-Ufer etc.

Gruppe F: Familiennamen, Herrscherhäuser, Volksstämme Bayardgasse, Im Dau, Quatermarkt, Steinbrecher Weg, Hohenzollerning, Hohenstaufenring, Salierring, Friesenstraße, Ubierring, Auf dem Hunnenrücken etc.

Gruppe G: Tier- und Pflanzennamen (Phytonyme)

Löwengasse, Schwalbengasse, Taubengasse, Habichtstraße, Ibisweg, Komoranweg, Neriveg, Holunderweg, Eibenweg, Buchenpfad, Eichenstraße etc.

Tabelle 3

Sektion II: Toponyme (Ortsnamen, Flurnamen, Gewässernamen)

Gruppe H: Ortsnamen (Oikonyme)

Kyotostraße, Turiner Straße, Tel-Aviv-Straße, Tunis-Straße, Tessiner Weg, Mülheimer Zubringer, Heidelbergweg, Indianapolisstraße, Gothaer Allee, Brühler Landstraße, Berlin-Kölnische-Allee etc.

Gruppe I: Flurnamen (Oronyme)

Am Bergerhof, In der Muckel, Am Frohnweiher, Zu den Bendengärten, Auf der Füllenweide, Siebenburgen, Weidengasse, Sandkaul, Zum Hedelsberg, Unter den Erlen, Unter den Ulmen, Unterste Sauerwiese etc.

Gruppe J: Gewässernamen (Hydronyme)

Rheingasse, Rheinuferstraße, Rothgerberbach, Auf der Rubr, Am Weidenbach etc.

Tabelle 4

Sektion III: Chrematonyme (Sach- und Objektnamen)

Gruppe K: Kirchen und Klöster

Antoniterstraße, Kartäusergasse, Minoritenstraße etc.

Gruppe L: Märkte und Nahrungsmittel

Alter Markt, Heumarkt, Holzmarkt, Neumarkt, Waidmarkt, Buttermarkt, Fiscbmarkt, Saligasse, Zur Kornkammer etc.

Gruppe M: Berufs- und Gewerbebezeichnungen

Faßbindergasse, Fleischmengergasse, Weberstraße, Seidmacherinnengäßchen, Unter Goldschmied, Unter Hutmacher, Unter Käster, Unter Taschenmacher etc.

Arbeitsanregungen: 
Finden Sie die angegebenen Straßennamen (Tab.2-4) auf dem Kölner Stadtplan. In welchen Stadtteilen liegen Sie? Können Sie eine geografische Ordnung erkennen?

Ordnen Sie dann die Straßennamen (Tab.2-4) bestimmten Motiven zu, beispielsweise dem Motiv Dichter. Können Sie die Motive noch einmal differenzieren, z.B. in deutsche Dichter und ausländische Dichter?

Finden Sie auf dem Stadtplan noch mindestens drei weitere Straßennamen, die zu den Motiven in den Tabellen passen.

Stellen Sie abschließend drei Namens-Persönlichkeiten Ihrer Wahl kurz vor.

Für einzelne der Arbeitsanregungen kann es sinnvoll sein, die Motivnamen in den Tabellen abzudecken (Projektor, Powerpoint) bzw. in den ausgeteilten Kopien auszulassen. Auch wäre denkbar, die einzelnen Straßen- und Wegenamen den Lernenden in einer beliebigen Reihenfolge vorzulegen, so dass diese dann eine systematische Ordnung nach Benennungsmotiven finden sollen. Umgekehrt könnte der Lehrende eine Tabelle mit den jeweiligen und dann noch weiter ausdifferenzierten Benennungsmotiven anbieten, für die dann anhand des Kölner Stadtplans Straßennamen-Beispiele gefunden werden sollen. Personennamen werden für die Lernenden höchstwahrscheinlich den größten Anreiz bieten, weil die Propria per se Identitätsbezüge herzustellen helfen.

$\mathrm{Zu}$ jeder Sektion ließen sich eigene Unterrichtseinheiten konzipieren. Dabei wären noch viele weitere Sub-Klassifizierungen denkbar, z.B. danach, ob es sich bei den Personen-, Familien-, Herrschernahmen um Kölner Persönlichkeiten handelt, ob diese weltweiten oder nur regionalen Bekanntheitsgrad haben etc.

\subsubsection{Praxis von Umbenennungen und mögliche Gründe}

Die Praxis von Straßenumbenennungen und ihre Gründe eignen sich hervorragend für eine Didaktisierung des Themas. In Neu- und Umbenennungen zeigt sich das Spannungsfeld von kultureller Erinnerung und sozialer Identiät am deutlichsten. Bering konstatiert: „Dabei ist natürlich das, was im Spiegel nicht erscheint, was also gezielt oder zufällig dem Vergessen zugeschrieben wird, ähnlich wichtig [...] wie das, was als namenswürdig angesehen wird.“" Die Frage „Können Straßennamen umbenannt werden und wenn ja: warum werden sie umbenannt?" ermöglicht viele interessante Antworten im Unterricht. Folgende Punkte können diese Erwartung stützen:

1. Zahlreiche Diskussionen um Straßenumbenennungen wurden und werden öffentlich in den Medien ausgetragen. Sie sind eben keine bloße Sache der Wissenschaft, sondern gehen jeden an, Fachmann wie Laien. Die Lernenden erkennen dadurch die Lebens- und Alltagsrelevanz des Themas. Zudem sind die Quellen der fraglichen Debatten oftmals online und damit leicht zugänglich.

10 Bering; Großsteinbeck 1994: 116 (Hervorhebungen im Original). 
2. Durch die enge Verankerung des Namens im Identitätsbewusstsein und der individuellen wie kollektiven Mentalität scheinen Straßennamen-Umbenennungen ein hohes emotional-psychologisches Konfliktpotential in sich tragen. Viele Auseinandersetzungen um Umbenennungen sind über Jahrzehnte mit einem erstaunlichen Elan und Esprit vor Gerichten ausgetragen worden, haben Politiker und Ämter beschäftigt etc. Mit einem Wort: Die Debatten sind alles andere als langweilig.

3. Kontroverse oder konsensfähige Debatten zu Umbenennungen von Straßennamen berühren direkt die geforderte Verzahnung von Sprach- und Kulturfragen. Die Diskussionen sind, mögen sie noch so abstrus erscheinen, immer auch ,Wortmeldungen des kulturellen Gedächtnisses. Straßennamen spiegeln die mentalitätsgeschichtlichen Wandlungen einer Gesellschaft wider. Wer etwas über die Kulturgeschichte von Straßennamen erfährt, erfährt immer auch Wesentliches über die Kultur und kulturelle Praxis ihrer Anwohner. Das prädestiniert das Thema geradezu für den Landeskundeunterricht.

4. Die Diskussionen zu Umbenennungen führen, dies war schon angesprochen worden, den Lernenden in ganz plastischer Weise vor Augen, dass die semantische Brisanz von Sprachfragen oftmals eine politische Brisanz impliziert oder evoziert, und dies nicht nur auf nationaler, sondern auch auf kommunaler Ebene. So sind die Konflikte um Straßenumbenennungen letztlich das, was man mit Michel Foucault „Diskurse der Macht“ auf allen Ebenen nennen könnte. Die Debatten zeigen, dass nicht nur nationale Sprachpolitiker und Sprachpfleger (etwa die „Académie française“ in Frankreich oder der „Verein für deutsche Sprache“ in Deutschland) massiv einzugreifen versuchen, wenn es um den vorgeblich schädlichen Einfluss von Anglizismen in der eigenen Sprache geht, sondern dass auch kommunale Politiker sich einmischen, wenn Bürger in hitzigen Leserbriefen etwa die Umbenennung der Kölner Heinestraße in eine Oscar-Wilde-Straße mit dem Hinweis zu verhindern suchen, dass dieser Straßenname dann "Oscar-Uwaild-Straße“ auszusprechen sei, denn: „Mister Wilde war kein Deutscher."11

\section{Entwicklung von Straßennamen im Mittelalter}

Straßennamen waren im Mittelalter, wie bereits ausgeführt, vor allem Teil des kommunikativen (Alltags-)Gedächtnisses der Sprecher und kein Teil des Straßenbildes; erst im 19. Jahrhundert wird das Namengut allmählich fixiert, indem Straßennamen an Häuserfassaden geschrieben werden (Köln: 1812) und im Zuge der großen Gesellschaftsdebatten und sozial-politischen Umbrüche des 18. und 19. Jahrhunderts eine ideologische Aufladung erhalten.

Der in Peter Glasners Dissertation angeführte Paradigmenwechsel der Straßenund Platzbenennungen vom Ortungsraum zum Mnemotop, also von einem reinen Abbildungsverhältnis zum kulturellen Erinnerungsort, kann für Unterrichts-

${ }_{11}$ ZAS (Zentrales Archiv für Straßenbenennungen) - Oscar-Wilde-Straße, Schreiben von Paul Sch. an das Vermessungs- und Katasteramt vom 6.10.1982. Zit. n. Werner 2008: 176. 
einheiten viel versprechende Ergebnisse zeitigen. Anhand weniger anschaulicher Beispiele lässt sich demonstrieren, wie unterschiedlich die Weltbilder und Weltansichten eines mittelalterlichen und eines modernen Menschen (gewesen) sind. Die Lernenden können Schritt für Schritt die zeit- und epochengebundene Relativität von Kultur- und Identitätsentwürfen erfahren, wozu die Tatsache gehört, dass die enge Bindung von Sprache und Kultur, von Sprache und Identität im kollektiv-nationalen Kontext im Wesentlichen ein Konstrukt des 19. Jahrhunderts ist, wenngleich mit Vorläufern bis in die Zeit des Barock. Wenn wir heute Begriffe wie „Leitkultur" verwenden, etwa in den Debatten um Thilo Sarrazins umstrittenes Buch „Deutschland schafft sich ab: Wie wir unser Land aufs Spiel setzen“ (Sarrazin 2010), so schwingt darin eine Geisteshaltung mit, die dem mittelalterlichen Menschen noch vollkommen fremd gewesen wäre.

\section{a) Bsp. Katharinengraben (Köln-Südstadt)}

Tabelle $5^{12}$

\begin{tabular}{|l|l|}
\hline Benennungsdatum & Straßenname \\
\hline \hline 1191 & secus Valum vetus \\
\hline 1251 & Supra Antiquuum fossatum \\
\hline 1317 & Super Antiqua fossa \\
\hline 1333 & $\begin{array}{l}\text { up der steede graven / aichter dem spitale von s. Katha- } \\
\text { rinen }\end{array}$ \\
\hline 1538 & Uff dem Altengraven \\
\hline 16. Jh. & Off S. Catreinen graben \\
\hline
\end{tabular}

Die topographischen Bezugnahmen schwanken zwischen Stadtgraben und Katharinenhospital. Glasner konstatiert: „Hieran wird deutlich, dass die mündlich tradierten Straßenbezeichnungen des Mittelalters aus der Wahrnehmung der Stadt entstehen und solange variieren, bis in der Sprechergemeinschaft der Stadtbewohner ein vorbewusster Konsens über die Namenfindung erzielt worden ist.“ (Glasner 2001).

12 Daten und Namen der Straßennamenentwicklung in den Tabellen 3-5 sind dem Aufsatz von Glasner entnommen, der unter anderem die Schreinsurkunden als Quellenbasis nutzt (Glasner 2001: 287 u.291). Dort finden sich auch weitere, detaillierte Hintergrundinformationen. 
b) Bsp. Salzgasse (Köln-Altstadt)

Tabelle 6

\begin{tabular}{|l|l|}
\hline Benennungsdatum & Straßenname \\
\hline 1135 & In vico Salis \\
\hline 1192 & In Salzgazzin \\
\hline 1307 & In Salzgassen \\
\hline 1542 & uff der Salzgassen \\
\hline 1571 & die salts gaß \\
\hline
\end{tabular}

c) Bsp. Hohe Straße (Köln-Zentrum)

Tabelle 7

\begin{tabular}{|l|l|}
\hline Benennungsdatum & Straßenname \\
\hline 1189 & Super stratam Lapideam \\
\hline 1260 & In Lapidea platea \\
\hline 1449 & Under spermecheren \\
\hline 1545 & Under Spermecher \\
\hline
\end{tabular}

Die beiden Straßennamen Saligasse und Hohe Straße exemplifizieren die Straßennamen-Praxis des Mittelalters. Diese war gekennzeichnet durch ein Ausprobieren und Verändern aufgrund gewandelter Wahrnehmungen, bis man allmählich zu einem Konsens gelangte, der aber nicht endgültig sein musste. Die Salıgasse wurde nach den dort ansässigen Salzhändlern benannt, während die Hohe Straße zunächst nach der damals seltenen Pflasterung (strata lapidea) und erst im Spätmittelalter nach den dortigen Speermachern ihren Namen erhielt, bis sie im Zuge eines längeren Wandlungsprozesses ihre heutige Benennung erhielt. Die folgenden Arbeitsanregungen könnte auf eine einführende Unterrichtseinheit zum Thema „die mittelalterliche Stadt" folgen, in der den Lernenden Grundwissen zum Beispiel zur Präsenz mittelalterlicher Handwerksberufe und Zünfte in den Städten des Heiligen Römischen Reiches deutscher Nation vermittelt wird. Köln als freie, unmittelbare und lange Zeit bevölkerungsreichste Handelsstadt des Reiches könnte hier wiederum als ein anschauliches Beispiel dienen. Es wäre aber auch denkbar, einen Unterrichtseinstieg in medias res zu wählen, mittels dessen die Lernenden intuitiv-assoziativ Gedanken zur Entwicklungsgeschichte ausgewählter mittelalterlicher Straßennamen in Köln kommunizieren. 
Arbeitsanregungen:

Schauen Sie sich die Entwicklung der Straßennamen des heutigen Katharinengrabens sowie der Salrgasse und Hohe Straße (Tab.5-7) an. Was fällt Ihnen auf? (Wie ändert sich die Sprache? Wie ist der Gebrauch der Lokalpräpositionen? Was wird bezeichnet?)

Finden Sie auf dem Kölner Stadtplan noch andere Straßen, die nach Handwerksberufen benannt sind und schreiben Sie diese auf. Welche der damaligen Berufe gibt es heute noch, welche sind mittlerweile ausgestorben?

1812 hielten die Franzosen unter Napoleon Köln besetzt. Sie beauftragten den Kölner Universitätsrektor Ferdinand Franz Wallraf damit, die damals nicht beschilderten mittelalterlichen Straßennamen neu zu benennen. Wallraf ordnete unter anderem an, die Pißgasse in Passage de la Bourse (Börsengasse), die Mördergasse in rue des mortiers (Mörsergasse) und die Bus(en)gasse in rue des Buisson (Buschgasse) umzubenennen. Warum hatte sich im Mittelalter wohl niemand an diesen Straßennamen gestört? Kennen Sie noch andere Straßennamen, entweder in Deutschland oder in Ihrem Heimatland, die Ihnen jemals negativ aufgefallen sind?

\section{Ideologisierung von Straßennamen im 19. Jahrhundert}

1881 wurde Köln von den Preußen zur Festung erklärt, was ein Bauverbot vor der alten Mauer nach sich zog. Aufgrund der Bevölkerungsentwicklung (1816: 50.000, 1880: 150.000 Einwohner) mussten die Stadtgrenzen ausgedehnt werden. Indem der Rat dem preußischen Minister Kamecke die alte Mauer und das Vorgelände abkaufte und Breschen in die alte Stadtmauer schlug, entstand die Kölner Neustadt. Eine unmittelbare Folge dieser Entwicklung war, dass 231 Straßen fast auf einen Schlag (neue) Namen erhielten, davon 23 noch in der Altstadt. ${ }^{13}$ Dieser einzigartige Fall für die historische Straßennamenforschung ließe sich für eine Unterrichtseinheit nutzen, in der die Lernenden, aufbauend auf ihre über die mittelalterliche Benennungspraxis gewonnenen Erkenntnisse, das neuzeitliche Prinzip der Historisierung und politischen Ideologisierung von Straßennamen herausarbeiten könnten. Die Stadt vor Augen sollte als Stadt im Kopf verankert werden, und zwar als „ein ideologisches Konstrukt: eine Selbstinterpretation, die in gezielt ausgewählter Vergangenheit Identität gewinnen will.“ (Bering; Großsteinbeck 1994: 110).

Die folgende Tabelle ließe sich im Anschluss an die Unterrichtseinheit zum Thema „die mittelalterliche Stadt“ einsetzen. Wiederum wäre beides möglich: Entweder den Lernenden eine kurze Einführung in die sozialen und politischen Verhältnisse im Deutschland der 1870er Jahre nach der Gründung des Deutschen Reiches sowie zur Rivalität zwischen dem von Preußen besetzten Rheinland und dem übermächtigen Preußen voranzustellen oder ihnen die folgende Kartografie

${ }^{13}$ Daten bei Bering; Großsteinbeck 1994: 108. 
gleich zu Beginn zu präsentieren und dann deduktiv zu verfahren. Wie bei allen Unterrichtseinheiten böte es sich auch hier an, dass die Lernenden ihre Ergebnisse in Arbeitsvorträgen vorstellen.

Tabelle 8 (vgl. Bering; Großsteinbeck 1994: 109)

\begin{tabular}{|l|}
\hline $\begin{array}{l}\text { Kartografie einer ideologisierten deutschen Geschichte am Rhein, } \\
\text { beginnend am Bayenturm in der Kölner Innenstadt/Südstadt (nach 1870) }\end{array}$ \\
\hline Ubierring (auch: Teuteburgerstraße) \\
\hline Chlodwigblatr \\
\hline Karolinger Ring (auch: Merowinger- und Rolandstraße) \\
\hline Sacbsenring \\
\hline Salier Ring \\
\hline Barbarossaplatz \\
\hline Hohenstaufen Ring (auch: Dassel- und Engelbertsraße) \\
\hline Habsburger Ring \\
\hline Rudolplatz \\
\hline Hohenzollern Ring \\
\hline Kaiser-Wilhelm-Ring \\
\hline Hansaring (auch: Hamburger , Lübecker-, Bremerstraße) \\
\hline Kulminationspunkt: Deutscher Ring (damals auch: Sedanstraße, Wörthstraße) \\
\hline
\end{tabular}

Arbeitsanregungen:

Recherchieren Sie im Internet die Bedeutung der neuzeitlichen Straßennamen (Tab.8) und vergleichen Sie diese mit den mittelalterlichen Straßenbenennungen (Tab.5-7). Welche Unterschiede und welche Gemeinsamkeiten fallen Ihnen auf? Was könnten die Gründe für die unterschiedliche Art und Weise von Straßenbenennungen im Mittelalter und in der Neuzeit sein?

Umbenennungen von Straßennamen während der NS- und in der Nachkriegszeit Alle diese Punkte lassen sich sehr gut am Beispiel der massiven Änderungen der Namen von Straßen, ja: ganzer Straßenzüge nach der nationalsozialistischen Machtergreifung von 1933 aufzeigen. Die neuen Machthaber versuchten, das Gedächtnis der Stadt von unliebsamen Mnemotopen und Erinnerungsinhalten sozu- 
sagen zu „reinigen“. Getilgt werden sollte vor allem die Erinnerung an die verhasste Weimarer Republik und an sozialdemokratische, kommunistische, liberal-freidenkerische und jüdische Persönlichkeiten. An die Stelle der alten Straßennamen traten nun die Namen der „Helden“ des Dritten Reiches wie z.B. Horst Wessel oder Dietrich Eckart.

$\mathrm{Zu}$ beobachten war zudem die Tendenz, das Kaiserreich nachträglich zu rehabilitieren, indem Straßen nach populären Generälen des Ersten Weltkrieges wie Hindenburg oder Ludendorff oder nach Repräsentanten der Hohenzollernmonarchie (Prinz-Heinrich-Straße) benannt wurden. Überhaupt ist eine Ideologisierung des Preußentums ein Hauptmotiv nationalsozialistischer Umbenennungspraxis. So musste der Erzbergerplatz 1933 wieder dem Königin-Luise-Platz, weichen (wobei dieser zehn Jahr zuvor durch den Namen Erzbergerplatz ersetzt worden war). Während der Zentrums-Politikers Matthias Erzberger, der 1918 die Kapitulation des Deutschen Reiches mit unterzeichnet hatte, von den Nationalsozialisten als Novemberverbrecher diffamiert wurde, zollte man der geradezu ikonisch verehrten Gemahlin des Preußenkönigs Friedrich Wilhelms IV. Respekt für ihren Versuch, bei Napoleon mildere Friedensbedingungen für das geschlagene Preußen zu erreichen. Dass beide, der Politiker und die Königin, letztlich nur eine außerhalb ihrer Verantwortung liegende militärische Niederlage bestätigen konnten, tat da nichts zur Sache.

Tabelle 914

\begin{tabular}{|l|l|l|}
\hline \multicolumn{2}{|l|}{ Straßenumbenennungen in Köln 1933-1958 (Auswahl) } \\
\hline vor 1933 & $1933-1945$ & nach 1945 \\
\hline Alte Wipperfürter Straße & Braunauer Straße (1938) & $\begin{array}{l}\text { Alte Wipperfürter Straße } \\
(1945)\end{array}$ \\
\hline Bebelplatz & $\begin{array}{l}\text { Dietrich-Eckart-Platz } \\
(1933)\end{array}$ & Bebelplatz (1945) \\
\hline $\begin{array}{l}\text { Deutscher Ring (bis } \\
\text { 1922); dann als } \\
\text { Teilstück: Platzder } \\
\text { Republik }\end{array}$ & Adolf-Hitler-Platz (1933) & Ebertplatz (1950) \\
\hline Erzbergerplatz & Königin-Luise-Platz (1933) & Erzbergerplatz (1945) \\
\hline Franz Hitze-Straße & Methfesselstraße (1939) & Franz-Hitze-Straße (1945) \\
\hline
\end{tabular}

14 Quelle: „Straßenumbenennungen in Köln von 1933-1939“ (http://www.gbg-koeln.de/denkmal/ns_zeit/str_33_39.htm). Diese von Schülerinnen und Schülern der Jahrgänge 9 und 11 erarbeitete Internetseite des Georg-Büchner-Gymnasiums in Köln liefert in ansprechendem Layout gute Hintergrundinformationen $\mathrm{zu}$ vielen Straßennamen und den historischen Namensgebern. Bereits Oebel 2006, 575 f., führt ähnliche Tabellen an. 


\begin{tabular}{|c|c|c|}
\hline Heinestraße & Corrensstraße (1935) & Heinestraße (1945) \\
\hline Hillerstraße & $\begin{array}{l}\text { Viktor-Schnitzler-Straße } \\
\text { (1933) }\end{array}$ & Hillerstraße (1945) \\
\hline $\begin{array}{l}\text { Innere Kanalstraße } \\
\text { (Köln-Nippes) }\end{array}$ & Ludendorffstraße (1938) & $\begin{array}{l}\text { Innere Kanalstraße (Köln- } \\
\text { Nippes) (1945) }\end{array}$ \\
\hline $\begin{array}{l}\text { Innere Kanalstraße } \\
\text { (Köln-Ehrenfeld) }\end{array}$ & Lisztmannstraße (1938) & $\begin{array}{l}\text { Innere Kanalstraße (Köln- } \\
\text { Ehrenfeld) (1945) }\end{array}$ \\
\hline Lassallestraße & $\begin{array}{l}\text { Prinz-Heinrich-Straße } \\
\text { (1933) }\end{array}$ & Lassallestraße (1945) \\
\hline Loreleystraße & $\begin{array}{l}\text { Ulrich-von-Hutten-Straße } \\
\text { (1936) }\end{array}$ & Loreleystraße (1945) \\
\hline Luxemburger Glacis & $\begin{array}{l}\text { Hannes-Miebach-Straße } \\
\text { (1939) }\end{array}$ & $\begin{array}{l}\text { Luxemburger Glacis } \\
\text { (1945) }\end{array}$ \\
\hline Mendelssohnstraße & Lis‡tstraße (1935) & Mendelssohnstraße (1945) \\
\hline Offenbachstraße & Brahmsstraße (1935) & $\begin{array}{l}\text { Brabmsstraße (1945); } \\
\text { Umbenennung der } \\
\text { Hannes-Miebach-Straße in } \\
\text { Lindenthal in } \\
\text { Offenbachstraße/ Offenbach } \\
\text { plat? (1958) }\end{array}$ \\
\hline Rathenauplatz & Horst-Wessel-Platz (1933) & Rathenauplatz (1945) \\
\hline Thywissenstraße & $\begin{array}{l}\text { Wilhelm-Gustloff-Platz } \\
\text { (1937) }\end{array}$ & Thywissenstraße (1945) \\
\hline
\end{tabular}

Arbeitsanregungen:

Finden Sie Gründe für die Um- und Rückbenennungen der Straßennamen und Plätze zu den angegebenen Zeiten (Tab.9). Welche Persönlichkeiten verbergen sich hinter den Namen? Recherchieren Sie (im Internet, in Bibliotheken etc.).

Nach dem Tod des Reichspräsidenten Friedrich Ebert am 2. März 1922 konnten sich der Rat der Stadt Köln nicht darauf einigen, einen Platz nach dem verstorbenen höchsten Repräsentanten der Republik zu benennen. Erst im Jahr 1950 erhielt der Ebertplatz seinen heutigen Namen. Welche Gründe kann es für die Weigerung des Rates gegeben haben? Recherchieren Sie dazu Hintergrundinformationen zur Person Friedrich Eberts und zur Weimarer Republik (politisches System, Gesellschaft, Wirtschaft etc.).

Umbenennungen von Straßennamen nach der Wende 
Der zweite einschneidende Wendepunkt der deutschen Geschichte nach dem Ende des Zweiten Weltkrieges, die friedliche Revolution und der Fall der Mauer von 1989, spiegelt sich auch in der Umbenennungspraxis von Straßennamen wider. Diese war oftmals von dem seinerseits nicht ideologiefreien Gedanken geleitet, die von kommunistisch-sozialistischen Ideologemen geprägte DDR-Erinnungskultur umzukodieren (Vgl. Kühn 2001; Azaryahu 1992). Die Denkmäler und Sprachbilder mit bestimmten, an den Kommunismus bzw. Sozialismus erinnernden Denotaten erhielten neue, unverfängliche oder eben anders verfängliche Namen. Die neue Sicht auf die Dinge veränderte das Gesicht der Stadt.

Wiederum ließe sich das Thema für einen an historischen Fragen interessierten Sprach- und Landeskundeunterricht nutzen. Eingebettet in eine Unterrichtseinheit zur Wendezeit könnte der Lehrende den DaF-Lernenden zum Beispiel die im Internet weitläufig dokumentierten kontroversen Debatten zur entweder erfolgten oder nach Protesten verhinderten Umbenennung von Leninstraßen, Ernst-ThälmannStraßen oder Karl-Marx-Plätz̧en in mehreren deutschen Städten präsentieren. Denkbar wäre auch ein schülereigener Arbeitsvortrag inklusive Erstellung einer Materialsammlung zur Geschichte der Um- und Rückbenennung einer ganzen Stadt: Chemnitz (bis 1953) $\rightarrow$ Karl-Marx-Stadt (1953-1990) $\rightarrow$ Chemnitz (nach einer Volksabstimmung vom 23. April 1990).

Am Beispiel des folgenden, insgesamt sechs Mal umbenannten Bolesłav-BierutPlatz in Magdeburg könnten die Lernenden wie an einem mnemischen Seismographen die jeweils veränderten Präferenzen von gelenkter Erinnerungskultur ablesen:

Tabelle $10^{15}$

\begin{tabular}{|l|l|}
\hline \multicolumn{2}{|l|}{ Umbenennung am Beispiel des Bolestaw-Bierut-Platzin Magdeburg } \\
\hline Benennungszeitraum & Straßenname \\
\hline $1885-1918$ & Kaiser-Wilhelm-Platz \\
\hline $1918-1922$ & Friedensplatz \\
\hline $1922-1933$ & Staatsbürgerplatz \\
\hline $1933-1945$ & Kaiser-Wilhelm-Platz. \\
\hline $1946-1951$ & Deutscher Platz. \\
\hline $1951-1989$ & Bolestaw-Bierut-Platz \\
\hline seit 1989 & Universitätsplatz. \\
\hline
\end{tabular}

15 Die Daten sind Kühn 2001: 308 entnommen. 
Arbeitsanregungen:

Zwischen 1885 und 1989 wechselte ein Platz in Magdeburg insgesamt neun Mal seinen Namen (Tab.10). Recherchieren Sie im Internet zu den jeweiligen Daten: Was ereignete sich in Deutschland während der angegeben Zeiträume (politisches System, historische Wendepunkte, soziale Zäsuren etc.)

Wer war Bolesław Bierut? Warum wohl benannte die DDR-Regierung einen Platz nach seinem Namen und warum wurde dieser Platz nach der Wende dann wieder umbenannt?

\section{Fallbeispiele Straßennamen: Carl-Diem-Weg und Heinrich-Böll-Platz.}

Um Näheres über die Umbenennungs-Praxis von Straßennamen in der Nachkriegszeit zu erfahren, wäre es möglich, sich zunächst didaktisch auf aussagekräftige Fallbeispiele zu konzentrieren, die geeignet sind, die Brücke von der alltäglichen Wahrnehmung zur Vergangenheit zu schlagen - spiegeln sich in den sehr kontroversen Debatten um diese Hodonyme doch zeitbedingte, sehr unterschiedliche Auffassungen zum Erinnerungswert der bezeichneten Personen und Objekte wider. Hierzu können die Lernenden umfangreiche Dokumentationen im Internet als Arbeitsgrundlage nehmen. Die beiden folgenden Fallbeispiele sind bei Werner (2008) anschaulich und umfassend dokumentiert. Hier gilt es, die dort aufzufindenden Ergebnisse noch einmal kurz zusammenzufassen und zu fragen, inwiefern sich eine Thematisierung der Beispiele für den DaF-Unterricht nutzen ließe.

\section{a) Bsp.1: Carl-Diem-Weg}

Die heftigen Debatten um den Namen Carl-Diem-Weg währten in Köln fast drei Jahre lang, und zwar von Januar 1994 bis November $1996 .{ }^{16}$ Zwischen den Fraktionen der Bezirksvertretung Lindenthal kam es zum Streit um den 1962 nach Carl Diem (1882-1962) benannten Weg. Schließlich hatte der Gründer und ehemalige Rektor der Deutschen Sporthochschule Köln im März 1945 auch vor einer Hitler-Jugend-Division zum „Opfertod für Vaterland und Führer“ aufgerufen. ${ }^{17}$ An der in der Lokalpresse breit dokumentierten Kontroverse beteiligten sich Bürger in zahlreichen Eingaben und Leserbriefen, die politischen Fraktionen des Rates der Stadt Köln, das Vermessungs- und Katasteramt, die Deutsche Sporthochschule sowie das Historische Archiv der Stadt Köln.

Man kam zu keiner abschließenden Entscheidung und ließ - nicht ganz untypisch für die Domstadt - alles beim Alten. Erst 2005 wurde die Debatte wiederbelebt. Die am vormaligen Carl-Diem-Weg in Köln gelegene Deutsche Sporthochschule unterlag schließlich im Rechtsstreit gegen die 2008 erfolgte Umbe-

\footnotetext{
${ }^{16}$ Erwähnt ist der Namensstreit bei Werner 2008: 6f.

17 „Kölner Stadt-Anzeiger“ vom 27.10.1995, „Zum ,Opfertod für Hitler` aufgerufen - Monitor zu Carl Diem“.
} 
nennung der Straße in Am Müngersdorfer Sportpark. Interessant ist auch, dass die öffentlichen Kontroversen um den in Würzburg geborenen Wahlkölner Carl Diem nicht auf Köln beschränkt blieben. Zuvor nach ihm benannte Straßen wurden auch in Mülheim an der Ruhr (1996), in Aachen (2007) sowie in Pulheim (2009) umgetauft. Grundschulen wie in Ritterhude, Hallen wie in Berlin-Steglitz oder in seiner Geburtstadt Würzburg erhielten ebenfalls andere Namen.

Folgende Quellen ließen sich nutzen, entweder für den Unterricht selbst oder für Referate von Arbeitsgruppen, welche die verwickelte Geschichte dieses Straßennamensstreits eingehender dokumentieren könnten:

Deutsche Sporthochschule Köln: Informationsmaterialien zur Diskussion um Carl Diem. (Pressematerialien zur Stellungnahme des Rektorats der Deutschen Sporthochschule Köln). Köln 1996.

„Kölner Stadt-Anzeiger“ vom 27.10.1995: „Zum ,Opfertod für Hitler aufgerufen - Monitor zu Carl Diem“"

„Kölner Stadt-Anzeiger“ vom 18.1.2002: „Grüne fordern neue Diskussion über Carl Diem“"18

„Kölner Stadt-Anzeiger“ vom 17.3.2005: „Erneute Diskussion um den CarlDiem-Weg“'19

„Kölner Stadt-Anzeiger“ vom 16.12.2005: „Carl Diem ist nicht länger Namensgeber" ${ }^{\prime 20}$

„Kölner Stadt-Anzeiger“ vom 21.8.2007: „Carl-Diem-Weg darf umbenannt werden“"21

„Kölner Stadt-Anzeiger“ vom 23.9.2009: „Carl-Diem-Straße wird umbenannt ${ }^{\text {"22 } 22}$

\section{b) Bsp.2: Heinrich-Böll-Platz.}

Die Kontroverse um die Verankerung des Namens des Kölner Literaturnobelpreisträgers Heinrich Böll (1917-1985) im Straßenbild seiner Heimatstadt sind besonders spannend, weil sie vor Augen führen kann, welche Ausmaße Diskussionen um Straßenumbenennungen annehmen können. ${ }^{23}$ Nur zwei Tage nach dem Tod des Schriftstellers am 16. Juli 1985 schlug die Bürgermeisterin Gepa Maibaum vor, die damalige Hülchrather Straße im Kölner Agnesviertel, an der Böll lange gelebt und gewirkt hatte, in Heinrich-Böll-Straße umzubenennen. Die Idee stieß auf geteiltes Echo. Als Alternativen wurden vorgeschlagen, entweder den Reichenspergerplatz oder den Appellhofplatz mit dem Namen des großen Sohnes der Stadt zu ehren. Gegen

\footnotetext{
18 http://www.ksta.de/html/artikel/1011285777239.shtml.

19 http://www.ksta.de/html/artikel/1109243515314.shtml.

$20 \mathrm{http}: / /$ www.ksta.de/html/artikel/1132657955723.shtml.

${ }^{21}$ http://www.ksta.de/html/artikel/1187683167324.shtml.

22 http://www.ksta.de/html/artikel/1246883941983.shtml.

23 Die Fakten zu diesem Namensstreit sind der sehr anschaulichen und gut lesbaren Studie von Werner entnommen: Werner 2008: 2-7.
} 
beide Vorschläge, einmal in der Lokalpresse publiziert, erhob sich ein Sturm der Entrüstung. Die Gegner stritten sowohl gegen eine Tilgung des an den Appellationsgerichtsrates August Reichensperger (1808-1895) erinnernden Straßennamens als auch gegen eine Umbenennung des Appellhofplatzes, der dem 1819 von den Preußen installierten „Rheinischen Appellationsgerichtshof“" seinen Namen verdankte. Eine von 331 Anwälten unterschriebene Petition forderte, „daß der Appellhofplatz, nicht umbenannt wird, sondern als Erinnerung an die liberale Rechtsprechung bestehen bleibt.“24 Ausgerechnet der Name des „PreußenGegners“ Böll, wie ein Schreiber im „Kölner Stadt-Anzeiger“ kritisierte, ${ }^{25}$ sollte die Erinnerung an eine Institution verdrängen, die mit rheinisch-französischer Liberalität ein Gegengewicht zur preußisch-rigiden Rechtsauffassung dargestellt hatte?

Ein Kompromissvorschlag der „Kölnischen Rundschau“, stattdessen den noch unbenannten Platz vor dem neuen Wallraf-Richartz-Museum/Museum Ludwig Heinrich-Böll-Platz zu nennen, hatte sich zunächst nicht durchsetzen können. Man monierte, dass diese Stelle nicht exponiert und prestigeträchtig genug für einen solch international renommierten Autor wie Böll sei. In der Sitzung der Bezirksvertretung am 17.10.1985 erreichte der Antrag der SPD-Fraktion, den Appellhofplatz in Heinrich-Böll-Platz umzubenennen, zwar Stimmengleichheit, wurde aber dennoch abgelehnt. Erst die Ratssitzung am 28. Januar 1986 fand zu einem wie es der „Kölner Stadt-Anzeiger“ formulierte - „Ende einer Blamage“.26 Einstimmig nahm der Rat in dieser Sitzung den Antrag an, den Platz vor dem neuen Museum am Dom nach dem Kölner Ehrenbürger zu benennen.

Folgende Quellen sind relevant, wiederum entweder für den Unterricht selbst oder für eine Präsentation der Straßennamen-Benennungsgeschichte durch die Lernenden. Sie sind alle schon bei Werner (2008: 344f.) aufgeführt. Leider liegen die Quellen aus Mitte der 80er Jahre nicht online vor, müssten also bei den Zeitungsverlagen bestellt, kopiert und ausgeteilt werden:

„Kölner Express“ vom 18.7.1985, „Heinrich-Böll-Straße fürs Agnesviertel!“ „Kölner Stadt-Anzeiger“ vom 13.9.1985, „Appellhofplatz wird Heinrich-BöllPlatz"

„Kölner Stadt-Anzeiger“ vom 17.9.1985, „Dem Preußen-Gegner ein Stück Preußen geopfert. Politiker zur Benennung des Appellhofplatzes nach Heinrich Böll““

„Kölner Stadt-Anzeiger“ vom 19.10.1985, „Lachen oder Weinen. Kein Platz für Böll““

${ }^{24}$ ZAS - Heinrich-Böll-Platz, Schreiben des Präsidenten der Rechtsanwaltskammer Köln (Dr. Heidland) an den Oberstadtdirektor Kurt Rossa vom 15.10.1985. Zit. n. Werner 2008: 4.

25 „Kölner Stadt-Anzeiger“ vom 17.9.1985, „Dem Preußen-Gegner ein Stück Preußen geopfert. Politiker zur Benennung des Appellhofplatzes nach Heinrich Böll“". Zit. n. Werner 2008: 2.

26 „Kölner Stadt-Anzeiger“ vom 29.1.1986, „Ende einer Blamage - Heinrich-Böll-Platz“. Zit. n. Werner 2008: 6. 
„Kölnische Rundschau“ vom 20.9.1985, „Wäre ein Namenswechsel im Sinne Heinrich Bölls?"

„Kölnische Rundschau“ vom 20.9.1985, Heinrich-Böll-Platz“ - Es gibt viele Möglichkeiten“

„Kölnische Rundschau“ vom 20.9.1985, „Museumsplatz für Böll: Nur einer legt sich quer"

„Kölner Stadt-Anzeiger“" vom 20.9.1985, „Reichensperger bleibt als Zeuge“

„Kölner-Stadt-Anzeiger“ vom 9.10.1985, Leserbriefe

„Kölner Stadt-Anzeiger“ vom 29.1.1986, „Ende einer Blamage - HeinrichBöll-Platz“

Arbeitsanregungen:

Carl Diem war ein verdienstvoller Funktionär in der Domstadt, Heinrich Böll ein international geehrter Kölner Autor. Warum kam es bei diesen Straßenumbenennungen dann überhaupt zu solch kontroversen Debatten?

Schreiben Sie einen Kommentar in einer Zeitung zur Umbenennung des Carl-Diem-Weges, in dem Sie entweder für oder gegen die Umbenennung Stellung beziehen.

Bilden Sie eine Diskussionsrunde zum Thema „Namensstreit um einen ,Nazie?", an dem sich folgende Personen beteiligen: Ein Vertreter der Kölner Sporthochschule, ein Journalist des „Express“, die Bürgermeisterin, ein früheres Mitglied der Hitler-Jugend, Anwohner des Carl-Diem-Weges, die Enkelin eines Opfers des Nationalsozialismus, ein Namensforscher etc..

Fallbeispiel (Fußball-)Stadionsnamen: Signal Iduna Park statt Westfalenstadion

Marion Werner hat am Beispiel der Kölner Toyota-Allee einen Paradigmenwechsel von Straßenbenennungen von einer kulturellen Motivation hin zu einer Kommerzialisierung nachgezeichnet - ein Phänomen, das die Semiotik vieler deutscher Städte zunehmend prägt (Werner 2008: 61-64).1994 hatte die Firma „Toyota“ einen Antrag an die CDU-Bezirksvertretung Lindenthal und an den damaligen Oberstadtdirektor gestellt, einen Straßennamen mit dem Namen der Firma zu erhalten. Das Kölner Zentrale Archiv für Straßenbenennungen (ZAS) lehnte diesen Antrag entschieden ab. Es schien, das Amt wollte verhindern, dass rein ökonomische Kräfte ihren Platz in der Symbolwelt des kulturellen Gedächtnisses reklamierten.

Zwar gab es bereits Straßen, deren Namen an Wirtschaftsunternehmen erinnerten, doch hatte man damit in erster Linie die Gründerväter der entsprechenden Unternehmen ehren wollen: Die Siemensstraßen in Neuehrenfeld und Porz sollten eben an Werner von Siemens (1816-1892), die Daimlerstraßen in Lövenich und Bickendorf an Gottlieb Daimler (1834-1900) erinnern. Diese Namen bedeutender deutscher Unternehmensväter sind im kulturellen Gedächtnis verankert. Wer aber kannte in Köln den Japaner Kiichiro Toyoda (1894-1952), der 1937 das 
gleichnamige Unternehmen gegründet hatte, das man im deutschen Sprachraum mit zwei „t“ schreibt? Trotz einiger Proteste wurde dem Antrag der Firma Toyota 1994 zugestimmt und dann 1999 endgültig entsprochen, die Straße, an der das Unternehmen seinen Standort hat, in Toyota-Allee umzubenennen. Werner konstatiert: „Von diesem Zeitpunkt an war Wirtschaftsförderung als Benennungsgrund legalisiert." (Werner 2008: 63). Der Paradigmenwechsel einer rein ökonomisch motivierten „Vertreibung der Kultur aus der Sprache“ (Ehlich 2006: 50) ist mittlerweile zur Normalität geworden: Heutzutage scheint es in vielen deutschen Städten beinahe opportun, Straßen oder andere städtische Signifikate nach ansässigen Firmen zu Werbezwecken zu benennen.

Die Analysen, welche die enge Verzahnung von Name und Identität und deren Gefährdungen belegen, müssen weder auf Köln noch auf Straßennamen beschränkt bleiben. Ein ebenso diskussionswürdiges Thema ist die seit Jahren zu beobachtende Tendenz, bestimmte Plätze der Begegnung und Erinnerung nach kommerziellen Gesichtspunkten umzubenennen. Ein besonders plastisches Beispiel für diese Praxis, Mnemotope durch „Kommerztope“ zu ersetzen, bilden Fußballstadien. Bering hat, unter anderem anhand zahlreicher Leserbriefe und Artikel in Zeitungen, dokumentiert, welche Auswirkungen die Umbenennung von Fußball- und allgemeiner: Sportstadien nach Wirtschaftsunternehmen oder Wirtschaftsprodukten auf die Identitätsversicherung der Fans haben kann. ${ }^{27}$ Früher traf man sich im Bielefelder Almstadion oder im Bochumer Rubrstadion, mittlerweile spielt man in der Scbüco Arena oder im rewirpowerSTADION. Das Hamburger Volksparkstadion wurde bereits so oft umbenannt, dass viele Fans den Überblick verloren haben und trotzig dem ihnen vertrauten Namen treu bleiben. Bis 3. Juli 2007 hieß das Stadion offiziell AOL Arena und bis einschließlich 30. Juni 2010 HSH Nordbank Arena. Zwischendurch erhielt es zur Fußball-Weltmeisterschaft den Namen FIFA WM-Stadion Hamburg, während die offizielle Bezeichnung zu den Europapokal-Spielen Hamburg Arena lautet.81 Jahre trafen sich die Kölner im Müngersdorfer Stadion, bis auch dieser Traditionsname 2004 fiel und durch RheinEnergieStadion ersetzt wurde - ein Name, der schon durch seine eigenwillige Schreibweise (ein Quasi-Kompositum durch fehlende Leerstellen zwischen den Substantiven) Aufmerksamkeit erheischt. Den Anhängern von Borussia Dortmund wurde gar der seit 1974 vertraute Name des Westfalenstadions entrissen und 2005 durch den Signal Iduna Park ersetzt. „Signal Iduna Park, niemals“ hatte noch ein wütender Schreiber in einem „Einwurf“ in der „FAZ“ am 14. Oktober 2005 standhaft verkündet und dabei offenbart, dass Namen durchaus nichts Profanes sind: „Namen sind nicht Schall und Rauch. Und der BVB gehört ins Westfalen-Stadion.

\footnotetext{
27 Bering 2007, v.a.461-464. Bei ihm findet sich nicht nur eine der ersten systematischen Aufarbeitungen dieses Themas, sondern auch eine tabellarische Einordnung der Namensumbenennungen von vierzig Stadien unter Nennung der jeweiligen Fußballvereine. Seine Tabelle eignet sich sehr gut als Grundlage für die Erstellung einer Materialsammlung für den DaF-Unterricht: Bering 2007: 438-441.
} 
Der Name des Fußball-Tempels ist heilig. So etwas verkauft man nicht - und wenn doch, dann ist Signal Iduna Park die Höchststrafe. " ${ }^{28}$

Zwei Monate später war die Namensbenennung unter Dach und Fach. Geschätzte 20 Millionen Euro, welche Borussia Dortmund von der Signal Iduna Gruppe bis 2016 erhält, waren letztlich doch überzeugender als mögliche Gefährdungen des kulturellen Gedächtnisses mittels rein kommerzgesteuerter Namensumbenennungen. Nach zahlreichen Protesten der Fans beschloss die Dortmunder Bezirksvertretung Innenstadt-West Anfang 2006 als eine Art Versöhnungsakt, zumindest eine Straße in Stadionnähe $A m$ Westfalenstadion zu nennen. Der Verein versuchte zwar zu intervenieren, weil er darin eine Spitze gegen den so wichtigen Sponsor sah, doch ging die Namensbenennung letztlich durch.

Bering hat den Prozess der Kommerzialisierung von Stadiennamen an den Beispielen Bochum (Rubrstadion $\rightarrow$ rewirpowerSTADION) und Nürnberg (Frankenstadion $\rightarrow$ easyCredit-Stadion) analysiert. Die bei ihm dokumentierten Artikel in der Lokalpresse bieten ausdrucksstarke Wortmeldungen von Seiten der Fangruppen und journalistische Kommentare, welche den hohen Identitätsgrad des vertrauten Stadionsnamens offen legen (Bering 2007: 449-458). Für den Namensstreit um das Westfalenstadion in Dortmund sind - als kleine Auswahl aus einer Vielzahl von Artikeln - folgende, sämtlich im Internet einsehbare Quellen interessant:

„FAZ“ vom 14.10.2005: „Signal Iduna Park, niemals. Ein Einwurf von Ralf Witzler"

„FAZ“ vom 6.9.2006: „Neue Stadiennamen ,schwer durchsetzbar“"29

„FAZ“ vom 1.7.2008: „Kommerzialisierung der Namenwelt. Gute Stimmung in der Aldi-Südkurve“‘30

Arbeitsanregungen:

Dokumentieren Sie die Geschichte der Umbenennung des Dortmunder Westfalenstadions in Signal Iduna Park.

Welche Argumente Pro und Kontra werden in den Artikeln angeführt? Kommen Sie zu einer eigenen Stellungnahme.

Stellen Sie sich vor, das Thema wird im WDR in einer Talkshow diskutiert. Bilden Sie dazu eine Diskussionsrunde. Wählen Sie verschiedene Diskussionsteilnehmer aus, die über das Thema diskutieren, z.B. Vertreter des Unternehmens Signal Iduna Group, des Fußballvereins Borussia Dortmund (Mannschaft, Trainerstab etc.), der Fangruppe, kommunale Politiker, ein Journalist.

\footnotetext{
28 „FAZ“ vom 14. Oktober 2004: „Signal Iduna Park, niemals. Ein Einwurf von Ralf Witzler“. http://www.faz.net/-00n26p.

$29 \mathrm{http} / / /$ www.faz.net/-00qd5y.

$30 \mathrm{http}: / /$ www.faz.net/-00tfpb.
} 


\subsubsection{Interkulturelle Vergleiche: Beispiel Valletta in Malta}

Abschließen will ich meinen Beitrag mit der Anregung, die Praxis von Straßenbenennungen in verschiedenen Ländern miteinander zu vergleichen. Natürlich würde auch die kontrastive Betrachtung von Straßennamen mehrerer deutscher Städte interessante Fragen evozieren, etwa die, warum es in so gut wie jeder deutschen Großstadt eine Beethovenstraße gibt, aber nicht überall eine Mendelssobnstraße, oder warum Düsseldorf 18,12 \% seiner Straßen mit Bezügen zu Kunst und Kultur benannt hat, während die selbst ernannte Kulturstadt (und gescheiterte europäische Kulturhauptstadt-Kandidatin) Köln in der Kernstadt auf gerade einmal 1,2\% solcher Straßennamen mit künstlerisch-kulturellen Denotaten kommt.

Für den interkulturell orientierten Landeskundeunterricht würde ein solch innerdeutscher Vergleich indes weniger effektiv sein. Hier gilt es, Kulturpraxis und Sprache von Ausgangs- und Zielkultur wechselseitig zu betrachten, und zwar unter Einbeziehung historischer Prozesse. Idealiter resultiert daraus eine Zunahme interkultureller Kompetenz, denn: „Ein tiefer gehendes Verständnis in Bezug auf die Entwicklung eines konkreten zielkulturellen Systems wird dann erreicht, wenn als kulturspezifisch erkannte Merkmale in ihren historischen Entwicklungszusammenhängen erklärt werden.“ (Bolten 2007: 96). Cultural Studies sowie interkulturelle Lernkonzepte im Landeskunde- wie im Sprachunterricht zielen darauf, sich mit mindestens zwei Kultur-Perspektiven reflektiv-kritisch auseinanderzusetzen. Krumm konstatiert: „Fremdsprachenunterricht muss prinzipiell interkulturell sein, insofern als sein Thema die Begegnung (die Konfrontation) mit einer anderen Sprache und Kultur ist.“ (Krumm 2003: 139). Und selbst für den reinen Forschungsbereich fordert Bering eine möglichst breite komparatistische Skala in der Straßennamenforschung: „Städte aus gleichem Raum und mit sehr ähnlicher (Sozial-)Geschichte müssten gegen solche gestellt werden, die ganz andere Lebensvoraussetzungen hatten." (Bering 2002: 212). Wo also gibt es Schnittpunkte, wo Differenzen? Es liegt natürlich nahe, dass die Lernergruppen Straßennamen aus den Städten bzw. Ländern wählen, die einer Mehrzahl der Lernenden vertraut sind. Es wäre darüber hinaus anzuraten, auch auf konfessionelle Bezüge der Lernenden Rücksicht zu nehmen und etwa im Falle einer Lernergruppe aus hauptsächlich islamischen Ländern eine Stadt auszuwählen, welche die religiösen Bezüge in ihrer Straßennamen-Praxis abbildet (und diese, dann oft, wie zum Beispiel im Falle der verfassungsmäßig laizistischen Türkei, nicht-religiösen Denotaten gegenüberstellt).

Die beiden letztgenannten Punkte werden die Straßennamen-Praxis im kleinsten und per Verfassung christlich-katholischen EU-Land Malta (410.000 Einwohner, 316 $\mathrm{km}^{2}$, Malteser zu 95 Prozent römisch-katholisch) nicht betreffen. Malta wähle ich dennoch aus, und zwar einmal aus ganz persönlichen Gründen, da ich am Department of German Studies an der dortigen Universität seit Anfang 2009 erster DAAD-Lektor bin und dabei auch mitgewirkt habe, einen MA-Studiengang zu 
gründen, der Elemente der Landeskunde, interkultureller Studien, aber auch der Pragma- und Soziolinguistik mit einschließt. Eine kulturell orientierte und kreativ arbeitende Fremdsprachendidaktik DaF ist hier besonders vonnöten, da Malta seine Bezugspunkte immer noch sehr stark am Nachbarn Italien und der ehemaligen Kolonialmacht Großbritannien ausrichtet. Deutschland ist dagegen vielen nach wie vor ein recht leerer Begriff, allenfalls angereichert durch semantisch eher dürftig konnotierte Stereotypen. ${ }^{31}$ Malta eignet sich darüber hinaus für eine kontrastive Analysen zu Köln, weil es in seiner alten Geschichte ähnlich wie die Domstadt seine christlichen, hier aber ausschließlich katholischen Identitätsbezüge in Straßennamen einschreibt - und dies meist im harmonischen Nebeneinander zu Straßennamen mit rein nationalen bzw. politisch-weltlichen Denotaten.

Schauen wir uns dazu einmal die Straßennamen in der Hauptstadt Valletta an, der kleinsten Hauptstadt Europas (ca. 6.100 Einwohner bei einer Fläche von $0.8 \mathrm{~km}^{2}$ ), die von dem französischen Großmeister des Malteserordens, Jean Parisot de la Valette (1494-1568), im Jahr 1565 unmittelbar nach dem erfolgreich abgewehrten Angriff des Osmanischen Reiches auf Malta nach strategischen Gesichtspunkten angelegt wurde. ${ }^{32}$

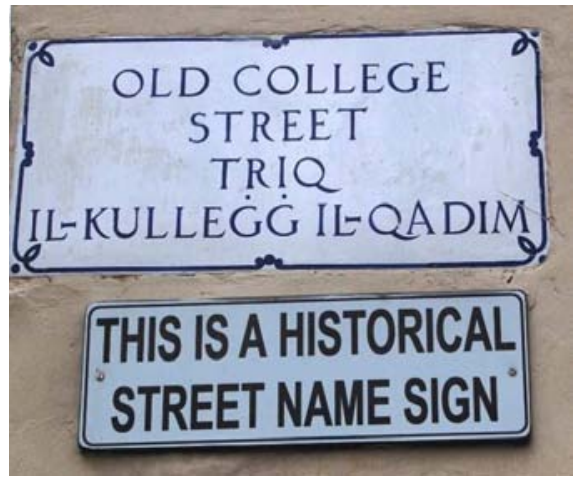

\section{Abb.4: „Historischer Straßenname“ in Malta, Englisch und Maltesisch Photo: Arndt Kremer.}

\footnotetext{
${ }^{31}$ Gleichwohl Malta lange zurückreichende kulturelle und politische Bezüge zu Deutschland aufweist: Der erste systematische Versuch, das Maltesische aus dem Punischen abzuleiten, unternahm 1718 der Deutsche Heinrich Johannes Maius; der letzte Großmeister des Malteserordens, Ferdinand von Hompesch, war ebenfalls Deutscher. Die deutsch-maltesischen Kontakte spiegeln sich dann auch vereinzelt in den Straßennamen wider. So findet sich im maltesischen St. Julians neben einer Triq Hans Stumme (benannt nach dem Orientalisten Hans Stumme, 1864-1936) auch eine Triq Albert Mayr (nach dem gleichnamigen Archäologen, der zu den maltesischen Tempelanlagen in Hagar Qim forschte). In Naxxar stößt man auf eine Triq Hieronimus Megiser, die an den Polyhistoriker und Sprachgelehrten Hieronimus Megiser (1554-1618/19) erinnert, der in seinen Werken „Polyglottus Thesaurus“ und „Propugnaculum Europae“ 1606 als einer der ersten maltesische Wörter sammelte und ins Deutsche übertrug. (Die Hinweise zu diesen Straßennamen verdanke ich meinem Kollegen Dr. Albert Friggieri).

32 Ein Straßenplan Vallettas findet sich z.B. unter: http://mappery.com/maps/Valletta-TouristMap.jpg.
} 
Es fällt auf, dass Vallettas Straßennamen nach einem Raster konzipiert wurden, das von einer mnemotopischen Planmäßigkeit geleitet zu sein scheint. Die Hauptstraßen, die weitgehend durchgängig vom Haupteingangstor zur Meerseite führen, tragen mit zwei Ausnahmen sämtlich Namen, die keine kirchlichen oder christlichen Denotate aufweisen: Republic Street (Maltesisch: Triq ir-Repubblika); Merchant Street (Triq il-Merkanti); Old Bakery Street (Triq il-Fran), Strait Street (Triq Id Dejqa), Old Mint Street (Triq Zekkea). Diese Straßennamen erinnern also erstens an die Unabhängigkeit Maltas von Großbritannien 1964 bzw. die Erklärung zur Republik 1974 (Republic Street), zweitens an die bis zu den Phöniziern zurückreichende Handelsgeschichte Maltas (Merchant Street) und drittens, ganz im Sinne der Analysen Glasners, an eine früher vorherrschende Tendenz zur Abbildung der städtischen Gegebenheiten durch Straßenbezeichnungen nach dort ehemals dominierenden Berufen (Bäcker: Old Bakery Street). Die angesprochenen Ausnahmen bilden die St. Ursula Street (Triq Sant' Orsla) und die St. Paul Street (Triq San Pawl), wobei sich die Platzierung der letzteren leicht durch das erwähnte Prinzip der Gleichwertigkeit von Signifikat und Signifikant erklären lässt, d. h. die Bedeutsamkeit des Namensträgers muss mit der - auch topologischen - Bedeutung des Straßennamens innerhalb der Stadt in etwa korrespondieren: Da Paulus als dem Schutzpatron Maltas ein exzeptionell hoher Symbolwert für die kollektive wie individuelle Identität der Malteser zukommt, wäre es insofern kaum vorstellbar gewesen, ihm in der Hauptstadt der Republik ein nicht ganz zentral gelegenes Bezeichnungsobjekt, also etwa eine Seitenstraße, zuzuweisen.

Diese vier Hauptstraßen mit von politischen, ökonomischen, christlichen bzw. beruflichen Denotaten gekennzeichneten Hodonymen werden nun aber gekreuzt von einer ganzen Reihe von Seitenstraßen mit Heiligennamen (Hageonymen) oder zumindest kirchlich-christlichen, das heißt im Falle Maltas ausschließlich katholischen Denotaten: St. John's Street (Triq San Gwann); St. Lucia Street (Triq Santa Lucia); Archbishop Street (Triq L'Arcisqof); St. Christopher Street (Triq San Kristoferu); St. Dominic Street (Triq San Duminiku) etc. Die Seitenstraßen führen in gerader Linie auf die Hauptstraßen zu, die auf dem Weg zur Seeseite wiederum zu großen Plätzen leiten, die an die Geschichte des Malteserordens erinnern: Great Siege Square (erinnernd an die erfolgreiche Verteidigung Maltas gegen den Angriff der Osmanen 1565) und Palace Square (an dem der Palast der ehemaligen Großmeister des Malteserordens steht, welcher heute zugleich Museum wie Präsidentenpalast ist).

Die Deutung liegt nahe, dass die Straßenbenennungs-Praxis in der Hauptstadt zeigen soll: Hier handelt es sich zwar um eine Republik mit ganz profanen politischen und ökonomischen Interessen, aber um einen Staat, der sozusagen sakral umrahmt ist, auch weil seine christlich-katholische Vergangenheit und Identität einen ganz wesentlichen Teil des kulturellen Gedächtnisses seiner Bürger bildet. Vallettas Gründervater, der Großmeister de la Valette, benötigt keine Straße mit seinem Namen - er ist im Namen selbst präsent, ist also dem kommunikativen und kollektiven Gedächtnis als Stadtname selbst unauslöschbar eingeschrieben. 
Die Abgrenzungsbewegung des katholischen Malta vom anglikanischen Großbritannien ist anhand einzelner Hodonyme besonders augenfällig abzulesen. Zwar übernahm Malta nach der Unabhängigkeit von der Krone 1964, die indes bis heute keinesfalls zu einem Austritt aus dem Commonwealth geführt hat, zunächst die meisten der britischen Straßennamen - dass jedoch ausgerechnet der Queensway in Pope Pius V Street (Triq Papa Piju V) umbenannt wurde, ist ein an Deutlichkeit kaum noch zu überbietendes Statement. Gleiches gilt für die Namensänderung der Britannia Street in Melita Street (Triq Melita), die an den alten, vielleicht ursprünglich phönizischen Namen Maltas erinnert. Anhand einer einzelnen Straße wie der heutigen Republic Street ließe sich zudem die moderne Geschichte Maltas im Kurzdurchlauf ersehen. Die Franzosen, auch in Köln verantwortlich für eine durchgängige Straßennummerierung $\left(, 4711^{\circ}\right)$ und eine ideologischere Straßenbenennungspraxis, gaben während ihrer kurzen Herrschaft über Malta unter Napoleon von 1798 bis 1800 der Hauptstraße den Namen Rue Nationale. Nach dem Abzug der französischen Truppen nannten die Briten die Straße in Kingsway um, der allerdings oft unter dem italienischen bzw. maltesischen Namen Strada Reale/Strada Rjali firmierte (Italienisch war bis 1933 Amtssprache Maltas). Nach der Trennung Maltas von der britischen Krone erhielt die zentrale Straßenader Vallettas dann ihren heutigen Namen. ${ }^{33}$ Ähnliche frankophone Umbenennungen fanden nach der französischen Besetzung von 1794 auch in Köln statt, wie sich an der wechselhaften Namensgeschichte der zentralen Plätze leicht erkennen lässt, die entweder eine völlig neue Bedeutung erhielten wie Neumarkt $\rightarrow$ place des victoires oder aber mit französischen Namen versehen wurden, die eine reine Bedeutungsübertragung aus dem Deutschen darstellen, so wie Freyheitsplatz $\rightarrow$ place de la liberté (vgl. Kramer 1993: 233).

$\mathrm{Zu}$ fragen wäre nun beispielsweise: Gibt es ähnliche Cluster in deutschen, vor allem katholisch geprägten Städten, z.B. in Köln oder München? In Valletta sind ca. $25 \%$ aller Straßennamen Hageonyme oder Namen mit kirchlichen bzw. religiösen Bezügen. In Köln waren es 1997 immerhin noch 13 \% religiöse Bezüge (Werner 2008: 283, Diagramm 37), zwar nicht ausschließlich katholischer Provenienz wie in Valletta, aber doch hauptsächlich. Vergleicht man die Straßennamenpraxis in Valletta und Köln, so ließen sich schon auf den ersten Blick Parallelen in der topografischen Orientierungsfunktion nach Clustern von Heiligen-, bzw. Kirchennamen erkennen. Die St Dominic Street in Valletta führt eben zur Dominikanerkirche, während die Kölner Cäcilienstraße zur Klosterkirche der Heiligen Cäcilie leitet. Dennoch lässt sich in Köln, z.B. in der Innenstadt, eine solch stringente Clusterisierung von Hauptstraßen, benannt nach weltlich-politischen Bezügen und darauf zuführenden Seitenstraßen mit kirchlich-religiösen Bezügen, nicht erkennen. Das mag daran liegen, dass die nationalpolitische Selbstvergewisserung der Republik Malta erst vor gerade einmal 40 Jahren wirklich konkrete Züge angenommen hat. ${ }^{34}$

33 Vgl. hierfür und generell als Fundgrube für die Recherche zu maltesischen Straßennamen: http://cilialacorte.com/Valletta $\% 20$ Street $\% 20$ Names.htm.

34 Natürlich zeigt sich auch in Malta das „mnemische Potential“ von Straßennamen bzw. der Benennungen öffentlicher Plätze besonders dann, wenn Umbenennungen anstehen - z.B. aufgrund 


\section{$5 \quad$ Fazit}

Straßennamen verbinden Alltagserfahrungen mit tiefer gehenden kultur- und mentalitätsgeschichtlichen Erkenntnissen und Gedächtnisinhalten. In der kontrastiven-internationalen Analyse lassen sich anhand von Hodonymen Parallelen und Differenzen in den Erinnerungsrepräsentationen verschiedener Gesellschaften und Nationen aufzeigen. All dies prädestiniert das Thema geradezu für einen handlungsbezogenen Sprach- und Kulturunterricht, der bestrebt ist, interkulturelle Kompetenzen zu fördern. Die Unterrichtsdiskussion sollte auf der Grundlage verschiedener Quellen geführt werden. Für die Quellenarbeit bieten sich in erster Linie Alltagsquellen wie Zeitungstexte inklusive Leserbriefen an, aber auch Archivmaterialien aus Zentralarchiven zur Straßennamenvergabe sowie Grund- und Adressbücher. Da viele Quellen im Internet zugänglich sind, können die Lernenden zur Eigenrecherche motiviert werden.

Die Einsatzmöglichkeiten von Straßennamen, aber auch von anderen Namensfeldern wie Objektnamen im DaF-Unterricht sind so vielfältig, dass hier nur einzelne Aspekte angesprochen werden konnten. Es wäre an der Zeit, dem Thema noch mehr Aufmerksamkeit zu widmen, z.B. durch die Erstellung einer kontrastiv ausgerichteten Materialsammlung von Quellen, welche die speziellen Erfordernisse des Fremdsprachen- und Landeskundeunterrichts berücksichtigt.

\section{Literatur}

Assmann, Jan (1992): Das kulturelle Gedächtnis: Schrift, Erinnerung und politische Identität in frühen Hocbkulturen. München: Beck.

Assmann, Jan; Hölscher, Tonio (Hrsg.) (1988): Kultur und Gedächtnis. Frankfurt/Main: Suhrkamp.

Azaryahu, Maoz (1992): Die Umbenennung der Vergangenheit, oder: Die Politik der symbolischen Architextur der Stadt Ost-Berlin 1990-91. In: Zeitschrift für Volkskunde 88 (1992), 16-29.

\footnotetext{
veränderter sozialer und politischer Präferenzen im Hinblick auf Erinnerungszeichen und -inhalte. Ein gutes Beispiel jüngeren Datums ist die Diskussion um die Umbenennung des Gaddafi Gardens (Gnien Gaddafi) im maltesischen Städtchen Paola. Was über Jahrzehnte niemanden wirklich interessierte, wurde nach dem brutalen Vorgehen Gaddafis gegen Oppositionelle während der Protestbewegungen und Kämpfe in Libyen im Sommer und Herbst 2011 Gegenstand längerer Kontroversen, nachzulesen u. a. in Zeitungsartikeln und Online-Kommentaren: Vgl. die OnlineKommentare zum Artikel „Gaddafi honours can only be removed by parliamentary resolution“, timesofmalta.com, 8.3.2011 http://www.timesofmalta.com/articles/view/20110826/local/parliamentary-secretary-proposes-renaming-of-gaddafi-gardens.381938). Vgl. den Kommentar zu dem Artikel "Libya no-fly zone would require bombing raids", timesofmalta.com, 1.3.2011 (http://daphnecaruanagalizia.com/2011/03/01/guess-whats-going-to-happen-if-somebody-daresmention-maltas-airfield/), Eintrag vom 2. März 2011: „Oh, and we need to change Gnien Gaddafi's name. Imagine if we had a Gnien Mussolini during World War II.“
} 
Bolten, Jürgen (2007): Interkulturelle Kompetenz. Erfurt: Landeszentrale für politische Bildung Thüringen.

Bering, Dietz; Großsteinbeck, Klaus (1994): Die Kulturgeschichte von

Straßennamen. Neue Perspektiven auf altem Terrain, gewonnen am Beispiel

Köln. In: Muttersprache 2 (1994), 97-117.

Bering, Dietz; Großsteinbeck, Klaus; Werner, Marion (1999): Wegbeschreibungen.

Entwurf eines Kategorienrasters zur Erforschung synchroner und diachroner

Straßennamenkorpora. In: Zeitschrift für germanistische Linguistik 27 (1999), 135-166.

Bering, Dietz (2001): Grundlegung kulturwissenschaftlicher Studien über

Straßennamen: Der Projektentwurf von 1989. In: Eichhoff, Jürgen; Seibicke,

Wilfried; Wolffsohn, Michael (Hrsg.) (2001): Name und Gesellschaft. Soziale und

bistorische Aspekte der Namengebung und Namenentwicklung. Mannheim u. a.:

Duden-Verlag, 270-281

Bering, Dietz (2002): Das Gedächtnis der Stadt. Neue Perspektiven der

Straßennamenforschung. In: Kremer, Dieter (Hrsg.) (2002): Onomastik. Bd. I.

Chronik, Namenetymologie und Namengeschichte, Forschungsprojekte. Tübingen:

Niemeyer, 209-225.

Bering, Dietz; Großsteinbeck, Klaus (2007): Die ideologische Dimension der

Kölner Straßennamen von 1870 bis 1945. In: Jaworski, Rudolf; Stachel, Peter

(Hrsg.) (2007): Die Besetzung des öffentlichen Raumes. Politische Codierungen von

Plätzen, Denkmälern und Straßennamen im europäischen Vergleich. Innsbruck: Frank

\& Timme, 311-335.

Bering, Dietz (2007): Die Kommerzialisierung der Namenwelt. Beispiel:

Fußballstadien. In: Zeitschrift für germanistische Linguistik 35, 3 (2007), 434-465.

Deutsche Sporthochschule Köln (Hrsg.) (1996): Informationsmaterialien zur Diskussion um Carl Diem (Pressematerialien zur Stellungnahme des Rektorats der Deutschen Sporthochschule Köln). Köln: DSHK.

Dinzelbacher, Peter (1993): Raum. In: Dinzelbacher, Peter (Hrsg.) (1993): Europäische Mentalitätsgeschichte. Stuttgart: Kröner, 604-615.

Ehlich, Konrad (2006): Die Vertreibung der Kultur aus der Sprache.13 Reflexionen zu einem reflexionsresistenten Thema. In: Zeitschrift für germanistische Linguistik 34 (2006), 50-63.

Föllner, Ursula; Luther, Saskia; Weinert, Jörn (Hrsg.) (2011): Straßennamen und Zeitgeist. Kontinuität und Wandel am Beispiel Magdeburgs. Halle (Saale):

Mitteldeutscher Verlag.

Frank, Rainer; Koß, Gerhard (Hrsg.) (1994): Namenkunde in der Schule. Reader zur Namenkunde IV. Germanistische Linguistik 121-123 (1994). Hildesheim u.a.: Olms. 
Glasner, Peter (2001): Vom Ortsgedächtnis zum Gedächtnisort: Straßennamen zwischen Mittelalter und Neuzeit. In: Eichhoff, Jürgen; Seibicke, Wilfried; Wolffsohn, Michael (Hrsg.) (2001): Name und Gesellschaft. Soriale und historische Aspekte der Namengebung und Namenentwicklung. Mannheim u. a.: Duden-Verlag, 282-302.

Glasner, Peter (2002a): Die Lesbarkeit der Stadt. Kulturgeschichte der mittelalterlichen Straßennamen Kölns. Köln: DuMont.

Glasner, Peter (2002b): Die Lesbarkeit der Stadt. Lexikon der mittelalterlichen Straßennamen Kölns. Köln: DuMont.

Humboldt, Wilhelm von (1830-1835): Über die Kawi Sprache auf der Insel Java. In: Leitzmann, Albert (Hrsg.) (1907): Wilhelm von Humboldts Werke, Bd.7. Berlin (Wilhelm von Humboldts Gesammelte Schriften, Bd.1-25), 1-344.

Jung, Udo O. H. (2000): Über das Kollektivgedächtnis der Deutschen und wie man sich die Zukunft verschafft. In: Info DaF 27, 6 (2000), 609-616.

Jung, Udo O. H. (2005): Straßennamen als kollektives Gedächtnis. In: Deutsch als Fremdsprache 2 (2005), 95-101.

Jung, Werner (2004): Das moderne Köln. Der historische Stadtführer. Köln: Bachem.

Kaufman, Fred; Lutz, Dagmar; Schmidt-Esters, Gudrun (Hrsg.) (1996): Kölner Straßennamen: Neustadt und Deutr. Köln: Greven.

Keller, Rudi (1994): Sprachwandel. Tübingen u. a.: UTB.

Klever, Alfred (2001): Köln. Eine Stadtgeschichte für junge Leser. Köln: Emons.

Korff, Gottfried (1992): Namenswechsel. Volkskundliche Anmerkungen zur „Politik“ der Straßenumbenennungen in der ehemaligen DDR. In:

Österreichische Zeitschrift für Volkskunde Bd. XLVI, 95, 325.

Koß, Gerhard (1990): Namenforschung. Eine Einfübrung in die Onomastik. Tübingen: Niemeyer.

Kramer, Johannes (1994): Französische Personen- und Ortsnamen im Rheinland 1794-1814. In: Dahmen, Wolfgang; Holthus, Günter; Kramer, Johannes; Metzeltin, Michael; Winkelmann, Otto (Hrsg.) (1993): Das Französische in den deutschsprachigen Ländern. Romanistisches Kolloquium VII. Tübingen: Günter Narr, 222-236.

Krumm, Hans-Jürgen (2003): Curriculare Aspekte des interkulturellen Lernens und der interkulturellen Kommunikation. In: Bausch, Karl-Richard; Christ, Herbert; Krumm, Hans-Jürgen (Hrsg.): Handbuch Fremdsprachenunterricht. Tübingen, Basel: A. Francke, 138-144. 
Kühn, Ingrid (2001): Umkodierung von öffentlicher Erinnerungskultur am Beispiel von Straßennamen in den neuen Bundesländern. In: Eichhoff, Jürgen; Seibicke, Wilfried; Wolffsohn, Michael (Hrsg.) (2001): Name und Gesellschaft. Soziale und historische Aspekte der Namengebung und Namenentwicklung. Mannheim u. a., 303-317.

Oebel, Guido (2006): Didaktisierung von Straßennamen aus dem deutschen Sprachraum für den interdisziplinären Unterrichtseinsatz - DaF, Germanistik, Landeskunde, Linguistik, Geschichte. In: Info DaF 33, 6 (2006), 569-583.

Peirce, Charles Sanders (1903): Pragmatism as a Principle and Method of Right Thinking. In: Turrisi, Patricia Ann (Hrsg.) (1997): Charles Sanders Peirce. Pragmatism as a Principle and Method of Right Thinking. The 1903 Harvard "Lectures on Pragmatism”. Albany, N.Y.: State of New York Press, 107-256.

Priebe, Ilona (2008): Kölner Straßennamen erzäblen: Von Schaafenstraße bis Filzengraben. Köln: Bachem.

Roche, Jörg (2008): Fremdsprachenerverb und Fremdsprachendidaktik. Stuttgart: UTB.

Sarrazin, Thilo (2010): Deutschland schafft sich ab. Wie wir unser Land aufs Spiel setzen. München: Deutsche Verlags-Anstalt.

Schmid, Angela (2005): Schildergasse, Alter Markt \& Co. Kölner Straßennamen und ibre Bedeutung. Köln: Compact.

Schultheis, Johannes; Walter, Hans (1968): Kritisches zur Straßennamengebung in Westdeutschland. In: Informationen der Leipriger namenkundlichen Arbeitsgruppe an der Karl-Marx-Universität 11, 7-9.

Werner, Marion (2008): Vom Adolf-Hitler-Platz zum Ebertplat:: Eine Kulturgeschichte der Kölner Straßennamen seit 1933. Köln: Böhlau. 


\title{
Der Erste Weltkrieg in populärliterarischen Medien
}

\author{
Joachim Sistig
}

\section{Einleitung: Unterrichtsthema „Erster Weltkrieg“}

Fremdsprachenunterricht soll über sprachliche und landeskundliche Zielsetzungen hinaus immer auch einen interkulturellen Anspruch erfüllen. Kommunikationskompetenz und Kulturwissen sollten niemals bloß Selbstzweck sein, solange nicht auch Fragen der Identitätsstiftung und historischen Verortung in Relation zu anderen Kulturräumen - besonders zum Herkunftshorizont des jeweiligen Lerners gestellt werden. Pierre Noras Arbeiten zu den unterschiedlichen Erscheinungsformen der „lieux de mémoire" Weltkrieg in Frankreich einen anderen, vor allem differenzierteren Stellenwert besetzen als in Deutschland: Neben den offiziell sanktionierten staatlichen Erinnerungsritualen mit Sarkozy und Merkel am 11. November auf den Champs-Elysées lässt sich durchaus auch ein Geschichtsbewusstsein „von unten“ gegenüber der „Grande Guerre“ beobachten, die im Bereich der Populärmedien z.B. durch die eindrucksvollen bandes-dessinées-Beiträge von Tardi einen unpatriotischen, antiautoritären, pazifistischen Kontrapunkt setzen. Deutschland scheint dem Ersten Weltkrieg gegenüber „geschichtsvergessen“. Kaum ein Erinnerungsort, kaum ein

\footnotetext{
1 Noras „Erinnerungsorte“ (Nora 1984, 1986 und 1992) sind ein epochales Grundwerk, das die französische Geschichtsschreibung als Erinnerungsforschung definiert, die sowohl einschneidende historische Ereignisse (Charlemagne) als auch Phänomene der Alltagskultur (Tour de France) gleichberechtigt betrachtet. „Lieux de mémoire” sind dabei nicht allein geografisch zu verstehen, sondern schließen auch Institutionen, Personen, Veranstaltungen u.a. ein, die Teil der kollektiven französischen „Gedächtniskultur" sind. In der Folge sind auch in anderen Ländern ähnlich angelegte Werke publiziert worden - in Deutschland: François; Schulze 2001, oder in Italien: Isnenghi 1996/1997.
} 
Ritual, kaum mehr eine Filmkulisse erinnern an diesen epochalen, mörderischen Konflikt, der im Kontext einer Jahrzehnte lang stilisierten „Erbfeindschaft“ vor allem ein deutsch-französischer Krieg war. Ernst Jünger und Erich Maria Remarque fristen im deutschen Literaturbetrieb eher ein Schattendasein. A la limite widmet ARTE hin und wieder einen Themenabend der Grande guerre inklusive einer der Verfilmungen von „Im Westen nichts Neues" von Lewis Milestone (1930) oder Delbert Mann (1979).

\section{Landeskunde}

Auch wenn der Erste Weltkrieg heute in Deutschland verdrängt scheint, hat er doch die Geschichte des Landes geprägt und seine Spuren hinterlassen: eine erste deutsche Revolution, die Einsetzung der parlamentarischen Demokratie, die politische Verankerung der Frauenrechte, die politische und wirtschaftliche Krise als Nährboden des aufkeimenden Nationalsozialismus usw. Um Deutschland zu verstehen, muss der Fremdsprachenlerner, der sich im DaF-Unterricht mit der deutschen Sprache und Kultur beschäftigt, notwendig auch dieses Geschichtskapitel studieren. Die zeitgenössischen Quellen und Kommentare sind nicht immer für den Fremdsprachenunterricht geeignet, da sie oft Faktenwissen voraussetzen, in einem aus heutiger Sicht altertümelnden Deutsch formuliert wurden und der Lebensrealität jugendlicher Lerner von heute auf antiquierte Weise entrückt erscheinen. Eine Unterrichtsreihe für eine DaF-Sequenz zum Thema „Erster Weltkrieg“" sollte daher auf einem gemischten Dossier aus unterschiedlichen Text- und Materialsorten basieren.

\section{Sprachvermittlung: Sprechanlässe, Sprachregister, Wortschatz, Grammatik}

Im vorliegenden Beitrag soll den populärliterarischen Textgattungen das Hauptaugenmerk gelten: Karikaturen und Bildgeschichten in Verbindung mit Fotos, Denkmal-Abbildungen und Liedern versprechen einen besonderen motivationalen Impuls. Der ironische Blick der Karikatur entlarvt den albernen bzw. tragischen Ernst der patriotischen Selbst- oder Fremdstilisierung. Die Semantik der Bilddarstellung ist offener als die geschichtliche Textdarstellung und regt eher zum spontanen Gedankenaustausch über ikonografische Details an. Im Sinne eines prétexte à la production orale sind bildliche Darstellungen ideale Stimuli für die Produktion von Sprechakten. Dekodierungsstrategien werden trainiert, die anschließend durch einen entsprechenden Aufgabenapparat begleitet in eine mündliche oder schriftliche Textproduktion münden sollen. Die Bildbeschreibung sollte von dem offenbaren oder versteckten Bildgegenstand, der Inszenierung des Dargestellten mit Vorderund Hintergrund sowie den sichtbaren oder auch abwesenden Hauptakteuren innerhalb der Vignette ausgehen. Eventuell lässt sich ein Vorlauf der Handlung bzw. eine Konsequenz der Aktion entwerfen. Es stellt sich weiterhin die Frage nach der intendierten Reaktion des Betrachters bzw. überhaupt nach dem inten- 
dierten Publikum, woraus Rückschlüsse über die politische Haltung des Zeichners selbst gewonnen werden können. Textteile - besonders Sprechblasen - können ausgespart bleiben und von dem Betrachter entworfen werden. Schließlich kann die gesamte Handlung in eine neue Textsorte projiziert werden - als Zeitungsartikel, Brief, Sketch usw.

Die Intention des Autors von Karikaturen und Bildgeschichten ist meist eine appellative, seltener verfolgt er ein rein poetisches oder referentielles Register. Der kritisch-ironische Blick soll überzeugen bzw. entlarven und den Betrachter zum Agenten der eigenen Überzeugung machen. Im Kontext des Ersten Weltkrieges geht es dabei zumeist um überzeichnete Freund- und Feindbilder. Die wörtliche Rede ist häufig in einem ,kumpelhaften“ Ton gehalten, die sich für die Beschäftigung mit syntagmatischen Wendungen aus der Alltagssprache anbieten. Gerade die Zeichnungen von Zille verweisen außerdem auf soziolinguistische Aspekte des „Milljöh“-Porträts im Berliner Wedding. Die plattdeutschen Redewendungen müssen teilweise erst entschlüsselt werden und bieten somit einen weiteren spielerischratenden Zugang zur Bild-Text-Botschaft - ebenso das altdeutsche Schriftbild, das für Ungeübte einer Entschlüsselung bedarf.

Die Grammatik steht bei einer landeskundlichen Thematik wie dem „Ersten Weltkrieg“" naturgemäß nicht im Vordergrund und sollte daher nur kursorisch einfließen, wenn sich ein Kapitel anbietet, weil spontan auf einen Fehler reagiert werden muss, oder weil ein Kommentartext explizit nach einer Übung ruft, wenn z.B. Zilles „Universal“-Artikel „dat“ einer präziseren Zuordnung bedarf. Zilles Bild-Kommentare - zumindest all jene aus seinen Berliner „Milljöh“-Studien könnten für sich genommen bereits als Grammatik-Übung verwendet werden unter dem Motto „Wer findet die Fehler?“ bzw. „Übersetzen Sie ins (Hoch)Deutsche!"6.

\section{Interkulturelle Kompetenz}

Unabhängig von der fachimmanenten Perspektive einer zielkulturellen Handlungskompetenz muss die erste Etappe eines interkulturellen Fremdsprachenunterrichtes darauf abzielen, Neugier bei den Lernern zu schüren. Dieser „,neu-gierige“ Blick sollte nicht allein von sprachlichen, ästhetischen oder landeskundlichen Fragestellungen geleitet sein, sondern eine echte interkulturelle Horizont-Erweiterung anstreben. Es soll hier nicht einer hybriden ,interkulturellen Misch-Identität“ als Unterrichtsziel das Wort geredet werden, wohl aber könnten auf diese Weise Weichen gestellt werden, um einen von nationaler Hybris erfüllten Abstammungsmythos als Leitidee individueller Identitätsbildung zu umgehen.

Am Anfang einer solchen Selbstreflexion müssen zunächst die Kulturunterschiede zwischen zwei Ethnien möglichst differenziert benannt und, anschließend, die zu Klischees geschrumpften Repräsentationen des Anderen diesen Realien gegenübergestellt werden. Abschottung, Ignoranz und Verschweigen von Klischees würden die Bereitschaft zur Verallgemeinerung hingegen erhöhen. Nur eine 
offene und reflektierte Konfrontation mit Auto- und Heterostereotypen hilft, diese zu überwinden, um am Ende ein tieferes Begreifen und Respektieren des Anderen zu erreichen. Im Rahmen dieser Reflexion sollten auch sozialpsychologische und politische Aspekte der Funktionalisierung von Fremdbildern erörtert werden. Die weitgehende Reduzierbarkeit des Fremdbildes auf seine Selbstaufwertungs- und Abgrenzungsfunktion wird den Jugendlichen den immanenten Zusammenhang von Fremd- und Selbstbildern vor Augen führen, wo die positive Selbsteinschätzung sich (fast) immer proportional zur negativen Fremdeinschätzung verhält.

Vier Themenfelder aus dem Umkreis des Ersten Weltkrieges sollen mit Hilfe von Karikaturen, Comics und Kurztexten erörtert werden - sei es als Stimulus für den Unterrichtseinstieg oder als stundenfüllende Materialgrundlage:

1. Fronterfahrung und Zivilleben im Ersten Weltkrieg mit einer Auswahl von Karikaturen von Heinrich Zille

2. Propagandistische Texte und Dokumente in der Schule und frühkindlichen Erziehung

3. Patriotische Texte und Darstellungen aus Feld- und Schützengrabenzeitungen

4. Ein Blick auf das grafische Schaffen von Walter Trier während des Ersten Weltkrieges mit einem Exkurs über seine tragische Rezeptionsgeschichte in Frankreich

Paradigmatisch soll die interpretatorische und ikonografische Auseinandersetzung mit den unterschiedlichen Materialien zeigen, welch sprachliches und faktisches Potential in den populärliterarischen Medien angelegt ist.

\section{Fronterfahrung und Zivilleben im Ersten Weltkrieg}

\section{Heinrich Zille (1858-1929): Vadding in Frankreich, 2. Folge (1917)}

In der politisch-satirischen Beilage des „Berliner Tageblatt“ erscheinen ab Kriegsausbruch Zilles Karikaturen des Frontalltages. Die Zeitungsbeilage trägt den Titel „Ulk“ („Unsinn, Leitsinn, Kneipsinn“) und wird von Otto Eyslers Verlag der „Lustigen Blätter“ publiziert. Zu seinen Mitarbeitern gehört übrigens nach 1918 u.a. auch Kurt Tucholsky. Unter dem Titel „Vadding in Frankreich“ erscheinen die rund 200 Bilder noch während des Krieges in gebundenen Heften. Der Duktus seiner Zeichnungen, die Motive seiner eigenen Militärdienstzeit zwischen 1880 und 1882 aufgreifen, sind von spontanem Mutterwitz, unorthodoxen Blickwinkeln, aber auch unbedingtem Gehorsam den Vorgesetzten gegenüber geprägt. Die Legitimation der deutschen Kriegsführung wird nicht in Zweifel gezogen. Seine Serien „Vadding in Ost und West“ sowie „Vadding in Frankreich“ (1. Folge) aus den Jahren 1915/16 spiegeln den politischen Mainstream mit patriotischen und auch 
kriegsverherrlichenden Motiven wider. Die autonomen, pazifistischen Beiträge seines nur in einer kleinen Auflage erschienenen Bandes „Kriegsmarmelade“ (1919) veröffentlicht Zille erst Jahre nach Kriegsende.

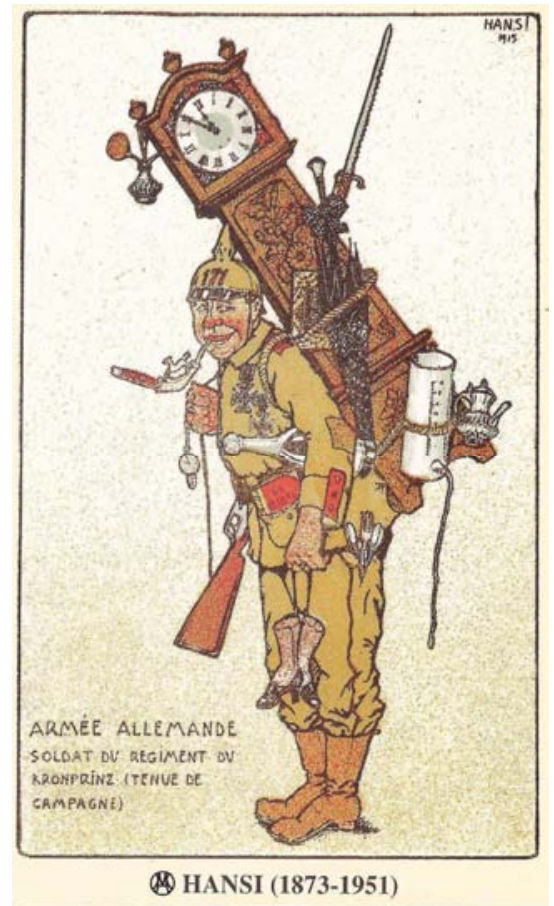

Abb. 1: Karikatur des elsässischen Zeichners Hansi.

Wenn der patriotische Impetus auch nicht so ausgeprägt ist, lässt sich doch eine Parallele zu den etwa zeitgleich publizierten Arbeiten des elsässischen Karikaturisten Jean Waltz, alias „Hansi“, ziehen (Abb. 1). Die Verlagsmitteilung im Klappentext des Vadding-Bandes unterstreicht überdies die semiotische Nähe der beiden Zeichenkünstler: „Man muß diese köstlichen, auch zeichnerisch glänzenden Bilder mit den geschmacklosen und giftigen Karikaturen des Westfranzosen Waltz, genannt Hansi, vergleichen, um rasch zu erkennen, auf welcher Seite mehr Freundlichkeit des Charakters und mehr ruhige Zuversicht zu finden sind. Dies Zille-Heft gehört zu den besten Erzeugnissen der Kriegsliteratur. Es wird auch nach dem großen Kampfe seinen Wert behalten.“2 Gegenüber dem idyllischen Stil Hansis, der immerhin in Berlin offenbar rezipiert wird, mit seiner eindimensionalen patriotischen Botschaft implizieren Zilles Zeichnungen hingegen spöttische Sozialkritik und politischen Zündstoff, der aber zu keinem Zeitpunkt diskursiv reflektiert wird.

${ }^{2}$ Berliner Tageblatt zitiert im hinteren Einband von Zille 1917. 
Zille porträtiert aus eigener persönlicher Erfahrung heraus in oft schroffen, ungeschönten Zeichnungen die soziale Lage des „Lumpenproletariats“ um 1900 in den Berliner Arbeiter-Hochburgen Wedding und Malchin, die er in leicht verklärender Weise zum Milljöh stilisiert. 1858 in Radeburg bei Dresden geboren zieht Zille neunjährig mit den wirtschaftlich ruinierten Eltern nach Berlin, wo er die sozialen und politischen Auswüchse der frühen Industrialisierung im direkten Umfeld seiner Familie miterlebt. Seine Zeichnungen, die Zille seit 1901 in der „Berliner Secession“ ausstellt bzw. im „Simplicissimus“, in „Jugend“ und in den „Lustigen Blättern" veröffentlicht, dokumentieren ungeschminkt die Lebensbedingungen der Unterschichten und sozialen Außenseiter. Zille verbindet seine Milieu-Porträts stets mit einem ironischen Blickwinkel, sodass die implizite soziale Anklage an Schärfe verliert und der Verdacht der Unterminierung bestehender Machtverhältnisse erst gar nicht aufkommt. Dennoch bieten all seine Arbeiten für den aufmerksamen Beobachter Ansatzpunkte für sehr genaue Sozialstudien der deutschen Gesellschaft um die Jahrhundertwende und während des Ersten Weltkrieges.

Drei ausgewählte Zeichnungen sollen jeweils Männer im Frontgeschehen und Frauen in ihren neuen Tätigkeitsbereichen zeigen. Jedes Bild ist von Zilles typischer Doppelbödigkeit gekennzeichnet. Hinter dem oberflächlichen - scheinbar harmlosen - Zeichencode verbirgt sich immer auch eine abgründige, pervertierende Bedeutungsschicht, die die zunächst vermutete Bildbotschaft wieder in Frage stellt:

\section{Kriegsszenen von der Front}

1) Cabarett Feldgrau

- „Du, Karl, kniep de elektrische Jungfrau nich in de Bein', dat is nämlich uns'e nigen Untroffzier!““ (Abb. 2) frei übersetzt: - „Du, Karl, kneif der elektrischen Jungfrau nicht ins Bein, das ist nämlich unser Unteroffizier!“ Zum Unterhaltungsprogramm der Frontsoldaten zählen neben Frontzeitungen, Witzblättern und kriegsverherrlichenden Comics vor allem Unterhaltungsveranstaltungen mit Cabarett-Charakter und Kirmesklamauk. Männer in Frauenrollen dienen als Ventil für die seelisch Verkrüppelten. Neben einschlägigen Zirkusfiguren tritt eine bärtige Person in Ballett-Toutou auf, die von Vadding - Zilles alter ego - sogleich in warnendem Ton identifiziert wird. Bei allem aufgesetzten Spaß bleibt die Veranstaltung doch eingerahmt von der schwarz-weiß-roten Reichsflagge als Symbol der immer noch gültigen Befehlsstrukturen. 


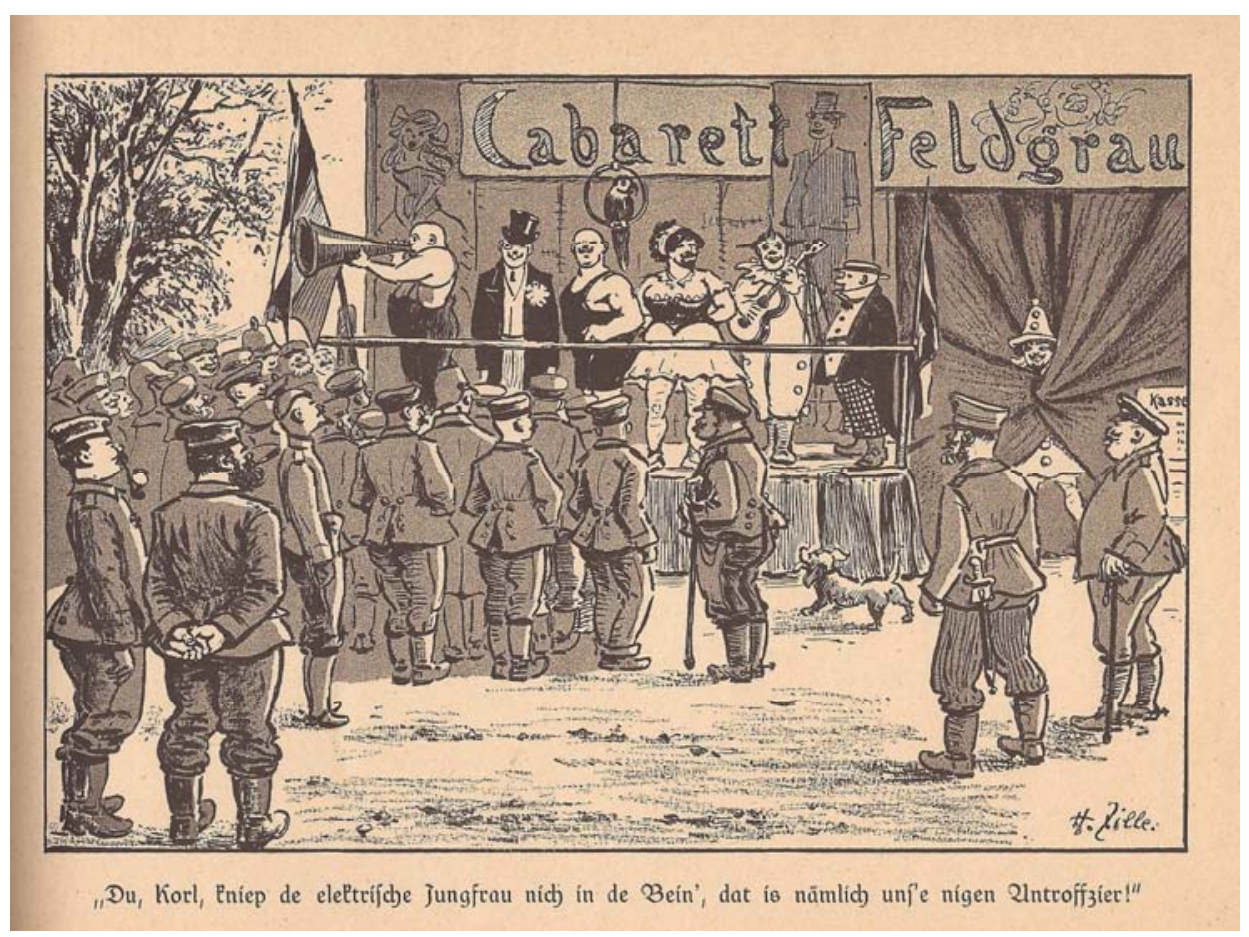

Abb. 2: Zille, Cabarett Feldgrau.

Der Spaß stößt also sehr rasch an seine Grenzen. Außerdem steht die „feldgraue“ Uniform in denkbarem Kontrast zum bemüht bunten Spektakel. Karl und Vadding sind mit genügend gesundem Menschenverstand ausgestattet, um auch in dieser scheinbaren Ausnahmesituation nicht den Respekt vor den Vorgesetzten zu verlieren.

2) Schützengraben-Idylle

- „Süh, Karl, wenn't sowiet is, nähmt wi uns'n Hindenburg-Park mit nah Hus!“ (Abb. 3); frei übersetzt: - „Schau mal, Karl, wenn es soweit ist, nehmen wir unseren Hindenburg-Park mit nach Hause!“ Ein Propaganda-Medium der Kaiserzeit ist die Umbenennung von Ortsnamen und die flächendeckende Aufstellung von Kaiser- und Bismarck-Denkmälern; Straßen, Plätze und Parkanlagen werden nach Persönlichkeiten und Orten mit patriotischer Konnotation umbenannt: Bismarckstraße, Kaiserpark, Kaiser-Wilhelm-Platz und Sedanwiese sind bis heute in fast jeder größeren deutschen Stadt zu finden. ${ }^{3}$ Einen Hindenburgpark können Hamburg, Ingolstadt, Köln oder Leverkusen aufweisen. Einige Grünanlagen musste der „Held von Tannenberg“ im Laufe der Jahre jedoch preisgeben, die in Volks-

${ }^{3}$ Vgl. den Beitrag von Arndt Kremer im vorliegenden Band. 
park (Berlin-Wilmersdorf), Friedenspark (Köln) oder Ebertpark (Ludwigshafen) umgetauft wurden. Die skurrile Idylle, die sich Karl und Vadding barfüßig um ihren Schützengraben geschaffen haben, wirkt gegenüber der relativ unscheinbaren Hindenburg-Büste in der Mitte etwas despektierlich.

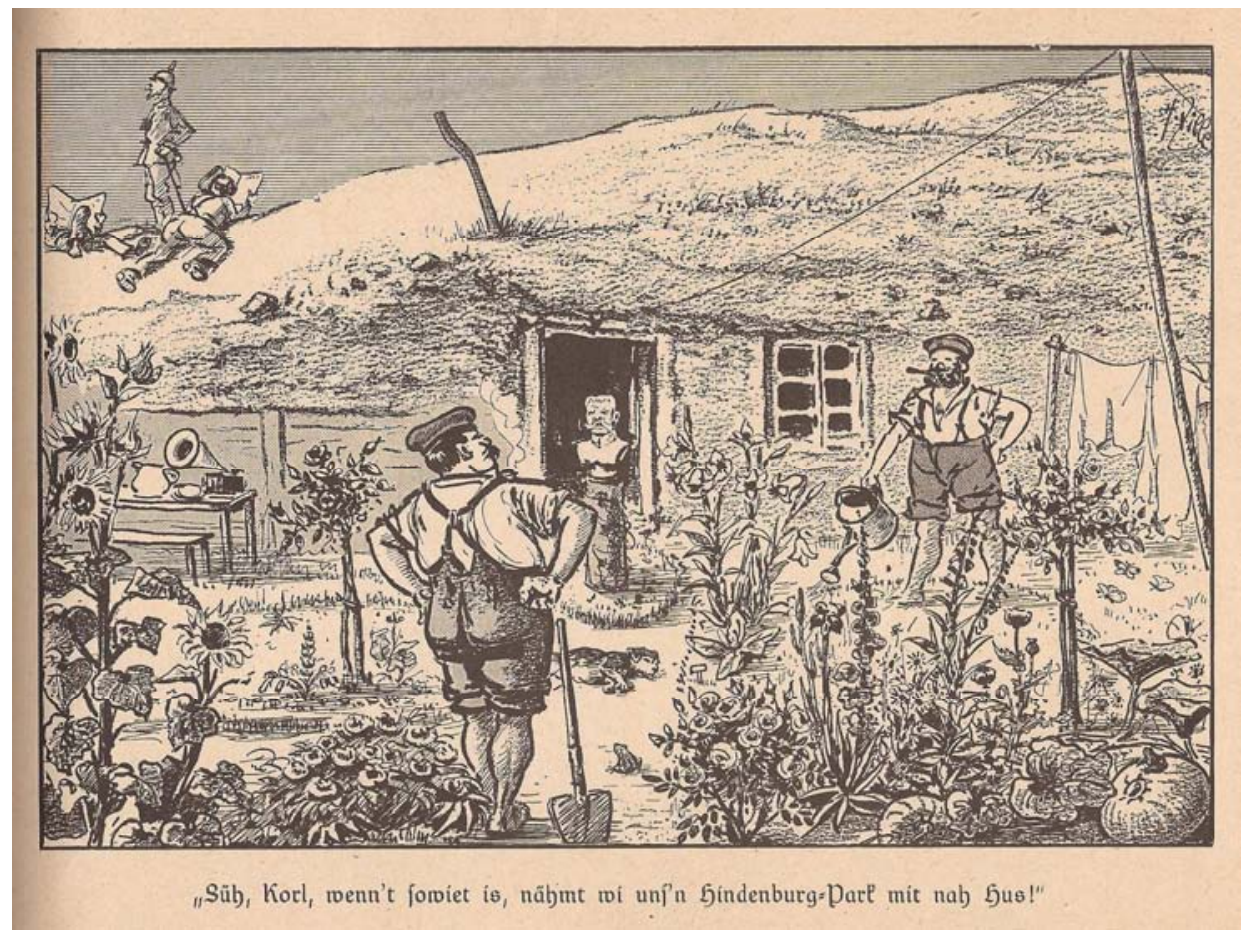

Abb. 3: Zille, Hindenburgpark

Ein schlafender Hund vor dem Denkmalsockel, die Wäscheleine direkt daneben und die dösenden Soldaten auf dem Graben geben der Bildbotschaft einen deutlich ironisierenden Charakter. Tatsächlich täuscht Zilles idyllische Schilderung des Frontalltags über die allgegenwärtigen Schrecken des Krieges durch Tod und Verstümmelung sowie menschenunwürdige Unterbringung, mangelnde Hygiene und grassierende Krankheiten hinweg:

Soldatenbrief

„24. Dezember 1916 Sainte Emilie; Weihnachtszauber! An mein liebes gutes Mutterl und Schwester! Heute Heiliger Abend, das Fest der Freude und Liebe! Wir hier sind im Kriegsgetümmel, sind Sklaven des Krieges, mit versteinerten Herzen und rauhem Wesen! Doch heute geht ein Weihnachtshauch durch aller Herzen! Also meine Lieben! Nur ein Wunsch: Frieden! Wir sind seit 
18. Dezember hier in St. Emilie, das Joseph gut kennen muss, denn er war damals im Oktober hier. Und zwar sind wir hier Divisionsreserve, morgen rücken wir nach Moislains und weiter, das damals so mitten im Schrecken der Somme-Kanonade lag, heute etwas ruhiger, und nach einigen Tagen weiter vor. Die meisten Verluste treten hier durch Erkrankung ein; ob ich's aushalte, wird sich zeigen. Man lebt natürlich das größte Dreckleben, wie ein Tier. Heute Sonntag und Heiliger Abend zugleich fingen wir an mit Dienst Exerzieren. Dann habe ich gepennt, und dann war in einer Scheune Weihnachtsgottesdienst um 6 Uhr für alle Konfessionen. Danach sangen wir einige Weihnachtslieder um einen Christbaum; liegen in der großen Zuckerfabrik. Im Übrigen ist Weihnacht für uns nicht da. Weichheit ist mir fern, wenn es auch unter den Leuten gärt, sie sich gerne gefangennehmen lassen wollen à la Verdun; ich werde mich wehren und das Vaterland mit dem letzten Blutstropfen verteidigen! Gruss und Kuss Euer Hugo." ${ }^{4}$

\section{3) Kontakt mit einheimischen Franzosen}

- „Na adschüs ok, Madam Quartierwirtin, un passens man up, dat ehr lütt Jean nich dat schöne Dütsch verlirnt, dat ick em bibröcht hew!“ (Abb. 4); frei übersetzt: - „Na dann, auf Wiedersehen auch, Frau Quartierwirtin, und passen Sie gut auf, dass der kleine Jean nicht das schöne Deutsch verlernt, das ich ihm beigebracht habe!“" Während die französische Propaganda von brutalen Übergriffen der deutschen Besatzungsmacht gegenüber der französischen Zivilbevölkerung berichtet hatte, zeichnet Zille ein verklärt harmonisierendes Bild der zwischenmenschlichen Kontakte. Vadding und Karl scheiden von der französischen Familie, bei der sie einquartiert waren, scheinbar als gute Freunde. Der soziale Wandel der Geschlechterrollen spiegelt sich auch in dieser Zeichnung wider: Als „Familienoberhaupt“ verabschiedet die Frau - „Madame Quartiermeisterin“ - die „Gäste“, während der Mann wie selbstverständlich abwesend an der Front gegen diese „Gäste“ einen tödlichen Kampf führt. Drei Generationen sind auf der pittoresken Terrasse unter dem respektvoll unangetasteten Insignium „RF“ (République Française) versammelt. Es hat anscheinend ein gemeinsames Abschiedsmahl gegeben, die Gesichter sind freundlich gezeichnet. Der alte Mann erhebt sich respektvoll - Karl und Vadding haben sich als höfliche „Gäste“ Respekt verdient. Die „,deutschen Spuren“, die Vadding glaubt hinterlassen zu haben, manifestieren sich nicht nur in der Vermittlung des deutschen Idioms an den kleinen Jean/Hans, sondern auch in Jeans typisch wilhelminischem Marinehemdchen, mit dem im Deutschen Reich bereits Jahre zuvor auf perfide Weise Werbung für die kaiserliche Flottenpolitik betrieben wurde. „Wie selbstverständlich“ ist jeder deutsche Knabe, dessen Eltern ihre rechte vaterländische Gesinnung zur Schau stellen wollen, zu Beginn des Jahrhunderts in die Militäruniform eines Marinesoldaten gezwängt.

4 Brief des Vizefeldwebels Hugo Frick an seine Familie bei Würzburg zit. n. Hirschfeld; Krumeich 2006: 149 . 


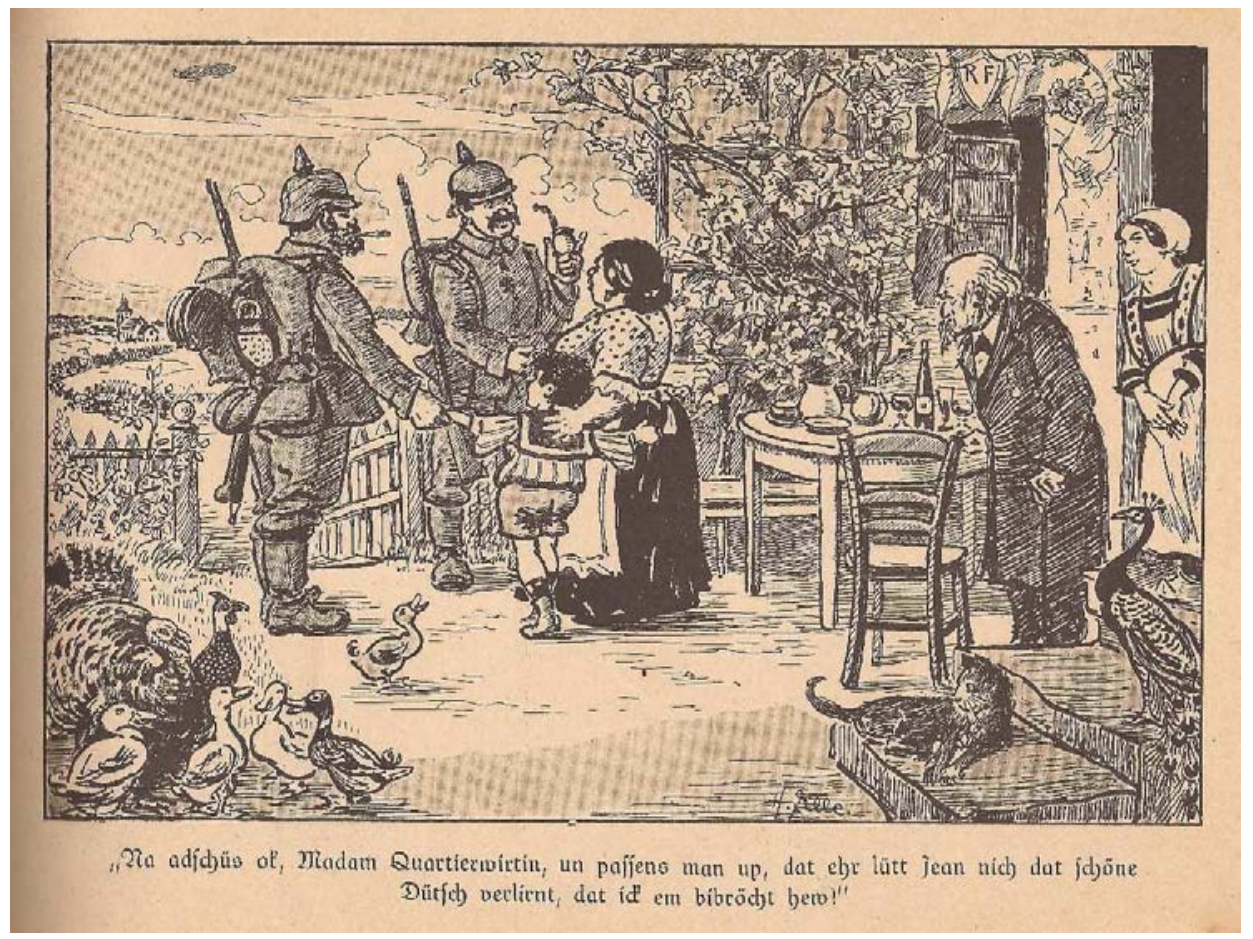

Abb. 4: Zille, Quartiersmadame.

Die Portion Selbstironie, die sich in der treuherzigen Selbstüberschätzung Vaddings bezüglich der Qualität seiner Deutschstunden mit „dat schöne Dütsch“ niederschlägt, soll den deutschen Soldaten zusätzlich Charme verleihen. Dennoch steht die sehr aufgesetzte Freundlichkeit im Kontrast zum Eisernen Kreuz an Vaddings Brust, das den französischen Zivilisten ständig den zahlreichen Tod vieler französischer Familienväter vor Augen führt.

\section{Kriegsszenen im Zivilleben}

Eine der Auswirkungen des Ersten Weltkrieges bezieht sich auf den Wandel der Geschlechterrollen. Während der Mann im Krieg seinen ,,vaterländischen Dienst“ verrichtet, übernimmt die Frau „Männer-Rollen“ im Zivilleben. Das Frauenwahlrecht von 1918 ist die politische Konsequenz der wachsenden Verantwortlichkeit der Frau in gesellschaftlichen Schlüsselpositionen. Nipperdey zeigt allerdings, dass der Erste Weltkrieg den tendenziellen Anstieg der Frauenlohnarbeit, der bereits seit 1895 feststellbar ist, lediglich verstärkt: „Tatsächlich tauchten immer mehr Frauen in ,klassischen' Männerberufen auf - ob in den öffentlichen Dienstleis- 
tungsbereichen, als Schaffnerin oder Briefträgerin, oder gar im Straßenbau oder der Munitionsherstellung."(Nipperdey 1998, Bd. 2: 798).

\section{1) Die Frau im Beruf 1}

- „Ja, Willem is seit acht Tagen eingezogen - un nu fahre ick seine Tour.“ - „Wat denn - als Schaffnerin? Wirste denn damit fertig?“ - „Na ob! Bei mir müssen se alle zahlen. Ick paß uff!“ (S.297) (Abb. 5).

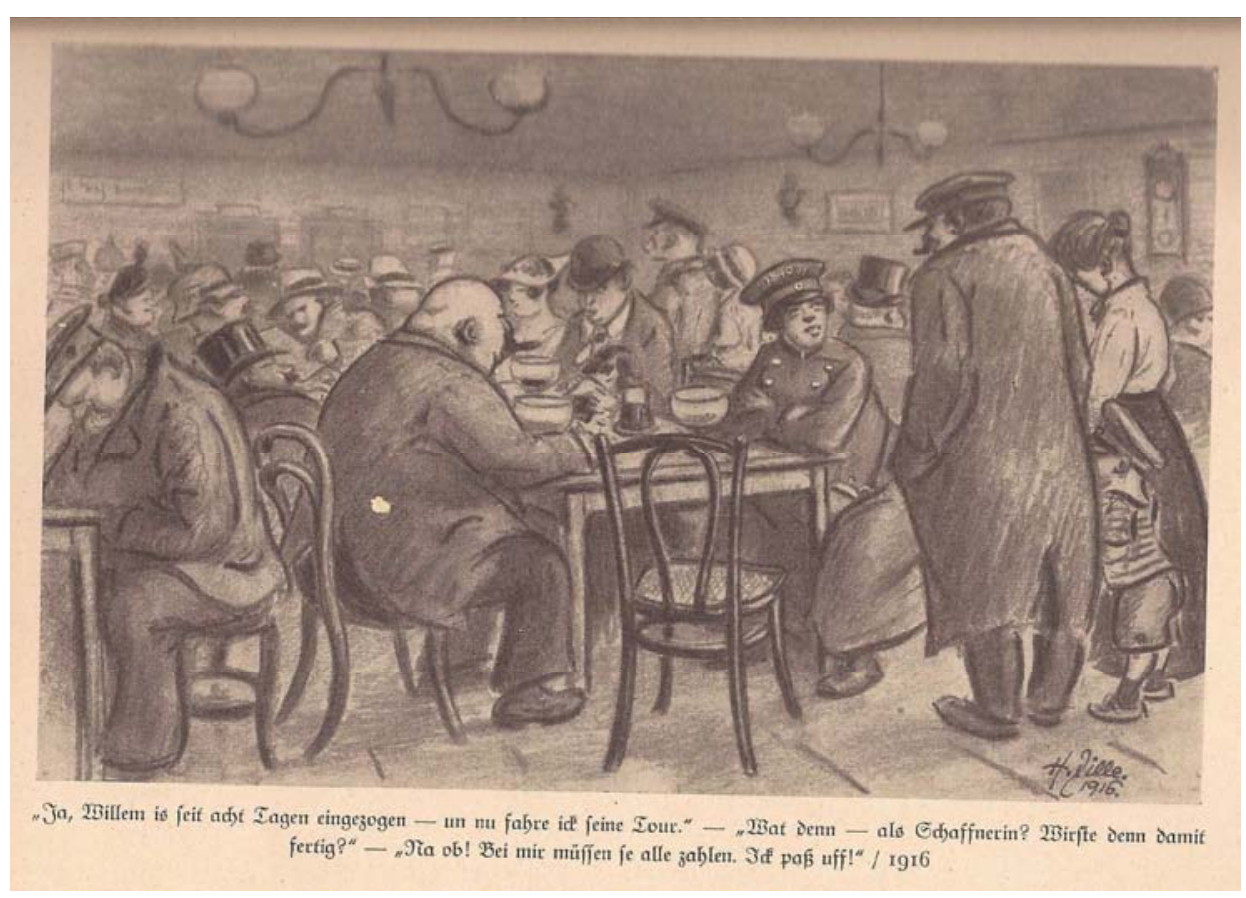

Abb. 5: Zille, Frauenrolle.

Zille inszeniert den Geschlechter-Rollentausch als paradoxe Sittenkomödie. Die Schaffnerin übernimmt nicht nur im Beruf die Rolle ihres an der Front dienenden Ehemannes Willem, sondern sie verkehrt auch in ihrer Freizeit in den gleichen „Etablissements“, obwohl in der Mehrheit Männer das Publikum bestimmen. Mit dem Kommentar „Bei mir müssen se alle zahlen“ gleitet Zille in eine zotige Konnotation hinüber, womit er andeutet, dass dieser Rollentausch für breite Teile der deutschen Gesellschaft immer noch eine anzügliche Wirkung impliziert. Die soziale Wirklichkeit eilt der moralischen Wertigkeit voraus. 


\section{2) Die Frau im Beruf 2}

- „Ick würde mir schämen, Frida, wenn ick mir so in Hosen zeigen sollte!“ „Warum denn? Haste O-Beine?“ (S.299) (Abb. 6). Die sehr detailreiche Straßenszene wirkt ohne speedlines statisch. Auch im Vergleich zu Wilhelm Buschs Bewegungssimulationen durch Konturvervielfachung fehlt es Zilles Zeichnungen an Dynamik. Seine Stärken liegen eher bei den Standbild-Einstellungen.

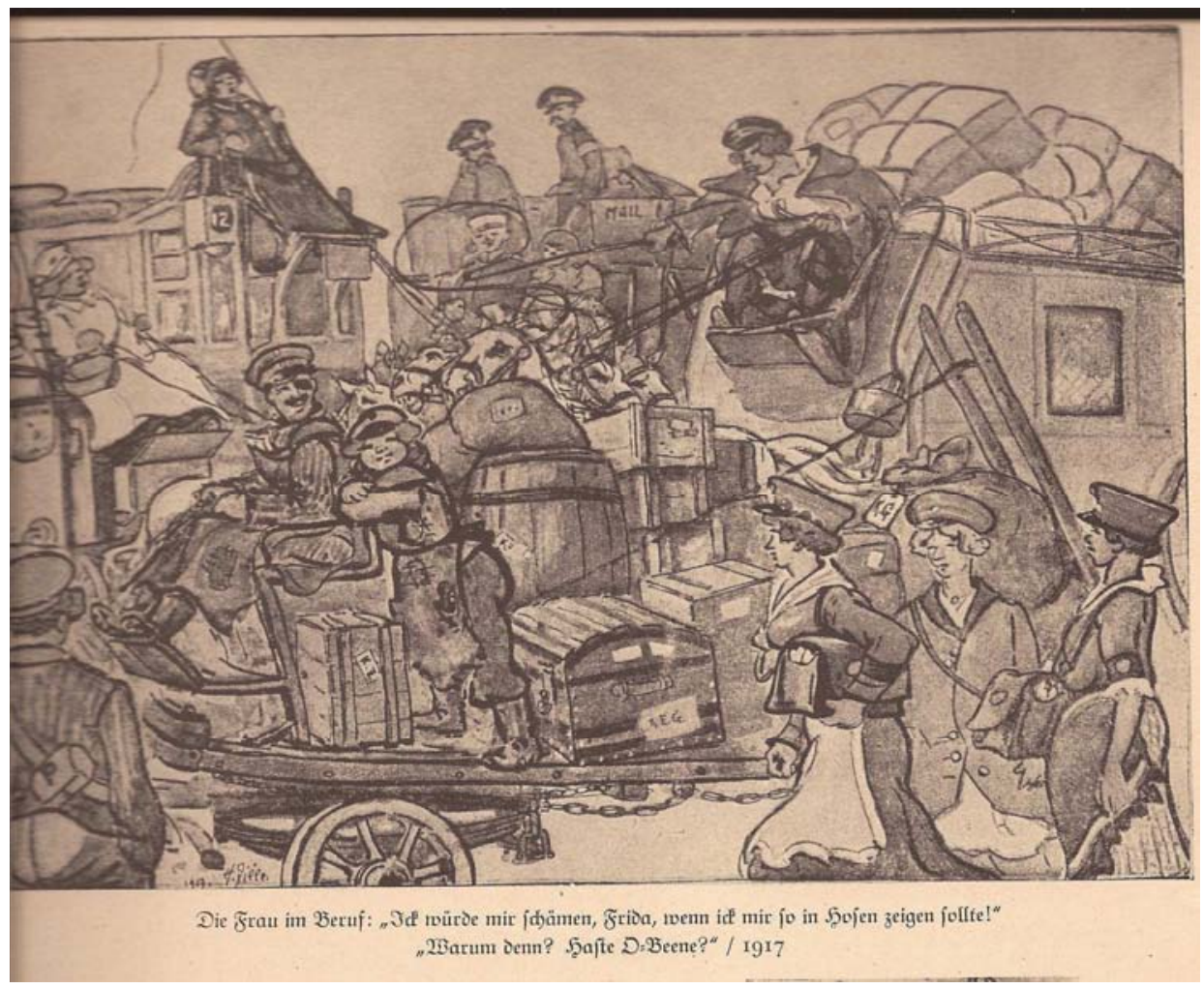

Abb. 6: Zille, Die Frau im Beruf.

Der Dialog, der dieser Szene unterlegt ist, spiegelt den moralischen Zwiespalt wider, in dem sich „Frau“ während des Ersten Weltkrieges bewegt. Das überkommene Sittenbild - „Ick würde mir schämen“ - aus Kaiserzeiten weist der Frau einen spezifischen nachgeordneten Platz zu, der klar von der Männerwelt getrennt ist. Kriegsbedingt muss diese Geschlechtertrennung aufgehoben werden. Diese neue Rollendefinition entspringt also nicht der Erkenntnis des Selbstverständlichen - „Warum denn?“ -, sondern ist den besonderen Zeitumständen geschuldet. Die Emanzipation ist nicht Ausdruck einer aufgeklärten, sondern einer pervertierten 
Gesellschaft, die aus den Angeln zu brechen droht, indem ihre konservativen moralischen Grundfesten in ihr Gegenteil verkehrt werden.

3) Schwarze Garde

- „Siehste, Juste, wir sind ooch wehrfähig: wir jagen ooch den Ruß fort!“ (S.300) (Abb. 7). Zille macht sich hier zum Sprachrohr des militarisierten Alltagsdiskurses mit seinen pervertierten Verwerfungen, indem menschenverachtende PropagandaParolen wie „Serbien muss sterbien. Jeder Stoß ein Franzos, jeder Schuss ein Russ!“ in den lapidaren Gesprächsfluss Eingang finden. Er dokumentiert damit, dass die „mühsam anerzogene Hemmschwelle gegenüber der Gewalt ${ }^{65}$ deutlich herabgesetzt worden ist.

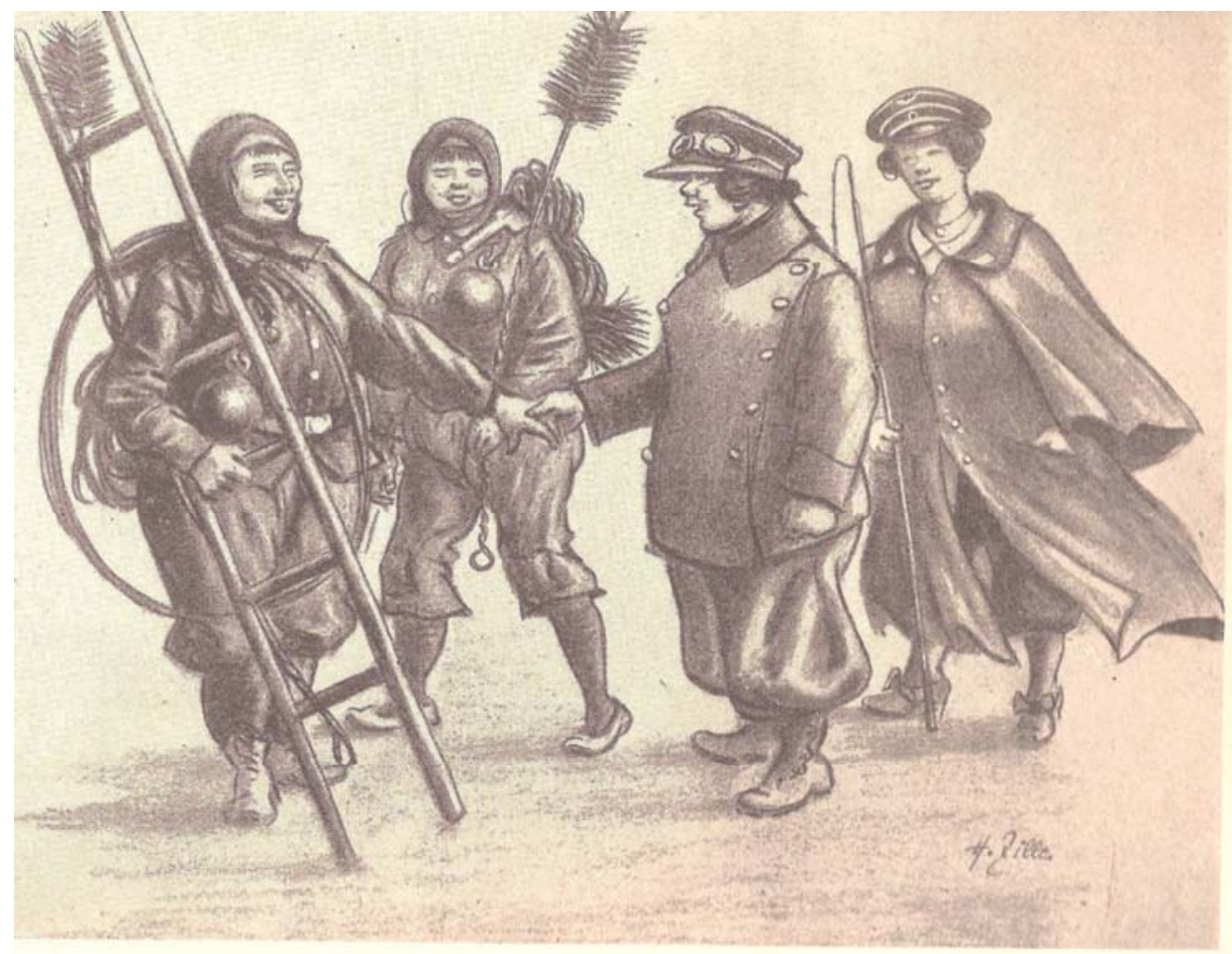

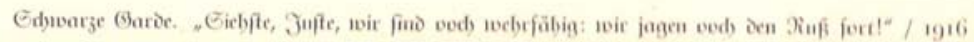

Abb. 7: Zille, Schwarze Garde.

Durch das Wortspiel mit der Klangähnlichkeit des Wortes „Ruß“ macht sich die militarisierte Zivilgesellschaft - die „schwarze Brigade“ - zu „wehrfähigen“ Mit- 
wissern und Mitkämpfern des Krieges im Osten gegen Russland. Das Bild der „schwarzen Brigade“ knüpft außerdem an den Mythos des 1. Leib-Husaren-Regimentes an, das in seinen schwarzen Uniformen und mit dem silbernen Totenkopf auf gekreuzten Knochen seit den Befreiungskriegen in der deutschen Kriegshistorie berüchtigt war.

\section{Militarisierte Kinderliteratur - militarisierte Kindheit}

Die Generalmobilmachung berührt im Kaiserreich alle Bereiche des öffentlichen Lebens und besonders auch den Schul- und Erziehungsbereich, indem gezielt Kinder zum Objekt der politischen Propaganda und der Erziehung zum Hass werden. Der Bildungsauftrag der Schulen war per Kaiser-Erlass definiert: „Bedenken Sie, was uns für ein Nachwuchs für die Landesvertheidigung erwächst. Ich suche nach Soldaten, wir wollen eine kräftige Generation haben, die auch als geistige Führer und Beamte dem Vaterlande dienen.“6 Im Zeichen der „schwarzen Pädagogik“, wie sie bereits in Heinrich Hoffmanns Struwwelpeter (1845) verbreitet wurde, erreicht die politische Propaganda längst auch die deutschen Kinderzimmer: Käthe Kruse fertigt z.B. seit 1914 eine Serie von uniformierten Stoffpuppen unter dem Namen „Potsdamer Soldaten“.

Heinz Lemmermann hat in seinem Referenzwerk zur „Kriegserziehung im Kaiserreich“ (1984) anhand zahlreicher Dokumente die „totale Politisierung der Schule“ im Sinne der Herrschaftsstabilisierung - im Kampf gegen den inneren Feind: die SPD - und der Kriegsvorbereitung - im Kampf gegen den äußeren Feind: Frankreich, England, Russland - nachgewiesen. Schullieder, Lernspiele und Kinderbücher bereiten die systemkonforme, ideologische Ausrichtung der Jugendlichen vor: „Der Krieg erscheint den Kindern als aktionsreicher, unterhaltsamer Vorgang. Das simulierte Töten durch Schießen, Schlagen und Niederstechen bietet nicht nur Spaß, sondern führt selbstverständlich zum Sieg. Die panmilitaristische Ausrichtung dieser lustbetont betriebenen Erziehungsmaßnahmen ist deutlich. $\mathrm{Zu}$ den soldatischen Fertigkeiten Marschieren, Exerzieren, Töten kommt in anderen Kinderliedern noch eine ideologische Komponente hinzu: Im Knaben soll so früh wie möglich das Bewußtsein geschaffen werden, es gar nicht abwarten zu können, bis er ein erwachsener Deutscher ist und kriegerischen Taten entgegenfiebern darf." ${ }^{7}$ Martin Kohlrausch hat nachgewiesen, dass die Neuausrichtung der Schulpolitik unter das Primat, „,nationale Deutsche‘ anstelle von ,jungen Griechen und Römern “ ${ }^{\text {8 }}$ hervorzubringen, auf den ausdrücklichen Willen des Kaisers zurückgeht. In einer an Stringenz armen politischen Führung insgesamt bildet die Erziehungsarbeit eine Ausnahme. Als Beispiel sei hier das „Soldatenlied“ (1878) von

6 Kaiser Wilhelm zu „Verhandlungen über Fragen des höheren Unterrichts“ am 31.10.1890 im preuBischen Kultusministerium, zit. n. Lemmermann 1984, Bd.1: 19.

7 Ebd., Bd.1, S.152.

8 Kohlrausch 2010: 62 . 
Hoffmann von Fallersleben (1798-1874), dem Schöpfer des Deutschlandliedes, zitiert, das vor allem in der Vertonung von Robert Schumann zu einem festen Bestandteil des kaiserlichen Schulliedkanons wird.

Soldatenlied

Ein scheckiges Pferd,

Ein blankes Gewehr

Und ein hölzernes Schwert,

Was braucht man denn mehr?

Ich bin ein Soldat,

Man sieht's mir wohl an,

Ich marschiere schon grad',

Halt' Schritt wie ein Mann.

Mit trotzigem Mut

Zieh' Morgens ich aus,

Kehr' freundlich und gut

Um Mittag nach Haus.

So wird exerziert

Zum Abend noch spat,

Bis der Schlaf kommandiert:

Zu Bett, Kamerad!

Eine Steigerung der wehrtüchtigen und ideologischen Mobilmachung ist Ludwig Nüdlings (1874-1947) Kinderlyrik unter dem Titel „Des deutschen Bübleins Wunsch" (1912):

Des deutschen Bübleins Wunsch

Mutter, warum bin ich noch so klein, Und nicht so groß wie des Nachbars Klaus

Denn der durft' in den Krieg hinaus.

Könnt' ich ein richtig Gewehr jetzt laden,

Spielte ich nicht mit Bleisoldaten.

Mutter, hier hast Du die Erbsen wieder,

Damit schießt man den Feind nicht nieder.

Lieber schlag ich die Sparbüchs entzwei,

Kaufe mir selber Pulver und Blei, und dann kämpf ich mit eigner Hand, Auf, für Kaiser und Vaterland.

Ach, wenn ich doch jetzt ein Zwilling wär, 
Gäb es gleich zwei Soldaten mehr, Und dann zög ich für's Deutsche Reich Gegen Franzosen und Russen zugleich, Einer zur Linken und einer zur Rechten, Könnt ich dann nach zwei Seiten fechten. Sähst von uns beiden dann keinen mehr, Bis der Weltkrieg gewonnen wär!

Die bedingungslose Hingabe für das vaterländische Ideal wird zum absoluten pädagogischen Hauptziel stilisiert. Der private Raum und das Individuum überhaupt werden dem Zwang des höheren Gemeinschaftszieles geopfert. Dass Ludwig Nüdling Priester im Bistum Fulda ist, steht keineswegs im Gegensatz zu dem hier propagierten, lebensverachtenden Patriotismus jenseits aller christlichen Ideale von Nächstenliebe und Barmherzigkeit. Die katholische und die protestantische Kirche haben sich noch vor Ausbruch der Kriegshandlungen dem völkischen Fanatismus im Deutschen Reich unter dem Motto „Für Gott und Vaterland“ begeistert angeschlossen. „Das Bewußtsein der gerechten Sache, die man verteidigt, aber auch der nationale Enthusiasmus und seine Hegemonieansprüche werden kirchlich gesegnet und überhöht. Superpatriotismus und Siegesfanfaren, Haß- und Rachegesänge, fast unbefangene Gleichsetzung von nationalen Zielen mit dem ,Reich Gottes', das erfüllt die Annalen. [...] Patriotische Pflicht und christliche Tugend, Bethlehem und Potsdam, das geht schnell ineinander über", urteilt Thomas Nipperdey - „Ein feste Burg' wird eine Art nationale Marseillaise.“ (Nipperdey 1998, Bd.1: 491).

Gedenk- und Gedächtnisfeiern in der schulischen Aula gehören zum festen Bestandteil des Schullebens im Kaiserreich. Gleich nach dem deutsch-französischen Krieg 1870/71 wird der nächste Krieg vorbereitet, indem die Jahrestage des Sieges von Sedan (2. September) und der Kaisergeburtstag (seit 1889 der 27. Januar) zum alljährlichen vaterländischen Jubelfest stilisiert werden. Seit Kriegsausbruch wird der Gedächtnis- und Totenkult an den Schulen gezielt intensiviert. „Die Flut der Kriegsfreiwilligen konnten die Behörden kaum bewältigen. Selbst Tertianer meldeten sich in Klassenstärke, es grassierte bei vielen Jugendlichen die ,Furcht', das Kriegs- und Bewährungserlebnis sowie den wahrscheinlicherweise raschen Sieg zu versäumen. “9 ${ }^{\text {}}$ Lemmermann aus dem Brief eines fünfzehnjährigen Rekruten an seine Eltern:

„Das Vaterland hat gerufen, und jeder waffenfähige Jüngling ist begeistert zu den Fahnen geeilt, um die heiligste Pflicht zu erfüllen. Geliebte Eltern, Ihr werdet die Gefühle eines von Vaterlandsliebe durchdrungenen Menschenkindes verstehen, um den Schritt, den ich unternommen habe, zu begreifen.

9 Lemmermann 1984, Bd. 1: 260. 
Du, lieber Vater, hattest den stolzen Ausspruch getan: wenn ich einen Sohn stellen könnte, würde ich stolz sein... “10

Das moralische Wertesystem ist vollends pervertiert und schickt seine eigenen Kinder in den sicheren Tod, weil die etablierten Moralinstanzen - Staat, Kirche, Schule, Familie - ihre Orientierung und Bestimmung verloren haben.

\section{Feldzeitung und Schützengrabenzeitung}

In den deutschen und französischen Schützengräben schaffen sich die Frontsoldaten mit ihren Kriegszeitungen, journaux de guerre bzw. journaux des poilus eigene Kommunikationsformen, die teilweise unterstützt, teilweise aber auch unabhängig von der Heeresleitung Informationen, Grußadressen, Comics, Karikaturen, Lieder, Witze und Kampfberichte aus der Feder der beteiligten Soldaten abdrucken. Diese oft amateurhaft improvisierten, manchmal sogar handschriftlich angefertigten $\mathrm{Pu}$ blikationen übernehmen die Funktion von ,sozialen Netzwerken“ avant la lettre. Oftmals multiplizieren ungeübte Zeichner und Autoren den Diskurs der offiziellen Propaganda in unterhaltsamen Bildern und Geschichten zum Zeitvertreib in der Schützengrabenödnis, spiegeln aber auch unterschwellig dissidierende Standpunkte und Stimmungen wider. Leider fehlt es bislang an neueren wissenschaftlichen Arbeiten, die aus historiografischer oder literarischer Perspektive diesen Fundus sichten. $^{11}$

Auf französischer Seite sind die Zeitungstitel meist fantasievoller - allen voran der mit dem bis heute unveränderten Logo arbeitende „Canard enchaîné“, der als Reaktion auf den extrem gewaltverherrlichenden „Poilu déchaîné“ entstand. Leicht defätistisch und mit geradezu surrealem Humor ausgestattet präsentieren sich u.a.

\footnotetext{
10 Ebd.

11 Die Universitätsbibliothek Heidelberg hat Teile der wöchentlich erschienenen Deutschen Kriegszeitung (Nr. 1 bis 20, August - Dezember 1914) aus ihrem umfangreichen Fundus an Frontzeitungen digitalisiert zugänglich gemacht (http://digi.ub.uni-heidelberg.de/diglit/feldztgdkz1914, http://digi.ub.uni-heidelberg.de/diglit/feldztgsunwas1917bis1918 [03.02.2011]). Verwiesen sei an dieser Stelle auch auf die eher methodologisch angelegte Diplomarbeit von Elke Daucher: Kriegszeitungen der Universitätsbibliothek Heidelberg. Überlegungen zu Erschließung, Erhaltung und Präsentation des Bestandes, Stuttgart 2003 (http://archiv.ub.uni-heidelberg.de/volltextserver/volltexte/2005/5838/pdf/Diplomarbeit.pdf [03.02.2011]); siehe auch: Hardt, Fred B.: Die deutschen Schützengrabenund Soldatenzeitungen, München 1917; Hellmann, Richard/Palm, Kurt: Die deutschen Feldzeitungen. Eine Bibliographie, Freiburg 1918; Kurth, Karl: Die deutschen Feld- und Schützengrabenzeitungen des Weltkrieges, Leipzig 1937. Auf französischer Seite wurde das Material der journaux du front weitgehender aufgearbeitet. Im Rahmen des „Gallica“-Projektes hat die Bibliothèque Nationale de France auch zahlreiche Frontzeitungen digitalisiert zur Verfügung gestellt (http://gallica.bnf.fr/Search?ArianeWireIndex=index\&p=1\&lang=FR\&q=journal +du+front [03.02.2011]). Neben der BN steht in der Bibliothèque de Documentation Internationale Contemporaine ein reichhaltiger Fundus digitalisiert zur Verfügung (http://www.bdic.fr/index.php?option$=$ com_content\&view $=$ article\&com_content $=\& i d=119 \& I t e m i d=101 \quad[03.02 .2011]$ ebenso wie in: http://www.caricaturesetcaricature.com/article-11180470.html [03.02.2011]); siehe auch: Tubergue; Charpentier 2007.
} 
„Le Bochofage. Organe anticafardeux, kaisericide et embuscophobe“ und „Rigolboche. Le journal le mieux renseigné sur les Teutons". Die Frontzeitungen entgehen nicht der militärischen Kontrolle - schon in der Nr. 7 des „Rigolboche“ vom 20. April 1915 taucht beispielsweise eine weiße Vignette mit den Worten „Dessin supprimé par la censure“ auf. ${ }^{12}$ Achim Schnurrer zeigt in seiner Studie, dass auf deutscher Seite spätestens seit 1916 mit Gründung der Feldpressestelle massive Eingriffe seitens der Militärzensur nachweisbar sind ${ }^{13}$.

Die Feldzeitungen, die in der Etappe hinter der Front gedruckt werden, erreichen bei hoher Druckqualität - gefördert durch die Heeresleitung - oft hohe Auflagen von mehreren tausend Exemplaren, während die Schützengrabenzeitungen in beweglichen Felddruckereien - aus disparaten Beiträgen eher zufällig improvisiert - selten wenige hundert hektographierte Einzelblätter überschreiten. Die „Champagne-Kriegszeitung“ beginnt beispielsweise zunächst in sporadisch erscheinender, manueller Schreibmaschinen-Qualität als Schützengrabenzeitung, bevor sie mit aufwändigen Tiefdruck-Illustrationen versehen zweimal wöchentlich in einer Auflage von bis zu 20.000 Exemplaren erscheint.

\section{1) „Der Drahtverhau“: Flugblätter!}

Diese „Schützengrabenzeitung des Bayerischen Landwehr Infantrie-Regimentes 1 “ (Abb. 8, S. 42) zeichnet sich durch unbedingte Kaisertreue aus, die sie noch 1917 durch eine Sondernummer „zu Kaisers Geburtstag“ dokumentiert (Nr. 16, 27.01.1917). Die Karikatur spielt mit dem Klischee von den „dumme boches“, die sich resistent gegenüber den französischen Aufklärungs-Versuchen zeigen. Trotz moderner Kriegstechnik - Flugzeug, Hangar, „psychologische Kriegsführung“ erreicht der Gegner doch nicht sein Ziel. Die Rückendarstellungen der beiden Soldaten im Halbprofil auf den zwei Zeichnungen lassen sich durch die Bärte und Uniformen dem deutschen und französischen Lager zuordnen. Die Missachtung des Flugblatt-Inhaltes durch seine Nutzung als Klo-Papier richtet sich als warnender Appell an die eigenen Truppen, die gegnerische Propaganda erst gar nicht zur Kenntnis zu nehmen. Die Zeit für inhaltliche Auseinandersetzung auf zivilisierter Ebene ist längst abgelaufen und eine Hinwendung zum Gegner durch Kenntnisnahme seiner Argumente käme Hochverrat gleich. Das Handeln wird gerade nicht mehr durch den Verstand geleitet, sondern durch primitive Instinkte und Bedürfnisse. Der Zielgruppe des „Drahtverhaus“ entsprechend sind die Dialoge in bayrischer Mundart formuliert: „Dö könn ma braucha, Michl! Dö schneid’n ma ausanand, na ham s' grad dö richtige Größ.“ Die deutschen Soldaten werden zu Sympathieträgern, indem sie mit positiv konnotierten Klischee-Elementen ausgestattet werden: Das Herz auf dem „Dixi-Klo“, die Pfeife, die aus der hinteren Hosentasche herausragt und der Hund, der sich die tierlieben, deutschen Soldaten und nicht deren Gegner als „Herrchen“ gewählt hat, stehen für „deutsche Gemüt-

12 Rigolboche, Nr. 7, 20.04.1915.

13 Schnurrer 2007. 
lichkeit“. Zuversicht soll vermittelt werden, dass deutsche Tugend und Charakterstärke gegen alliierte Technik und Heimtücke siegen werden.

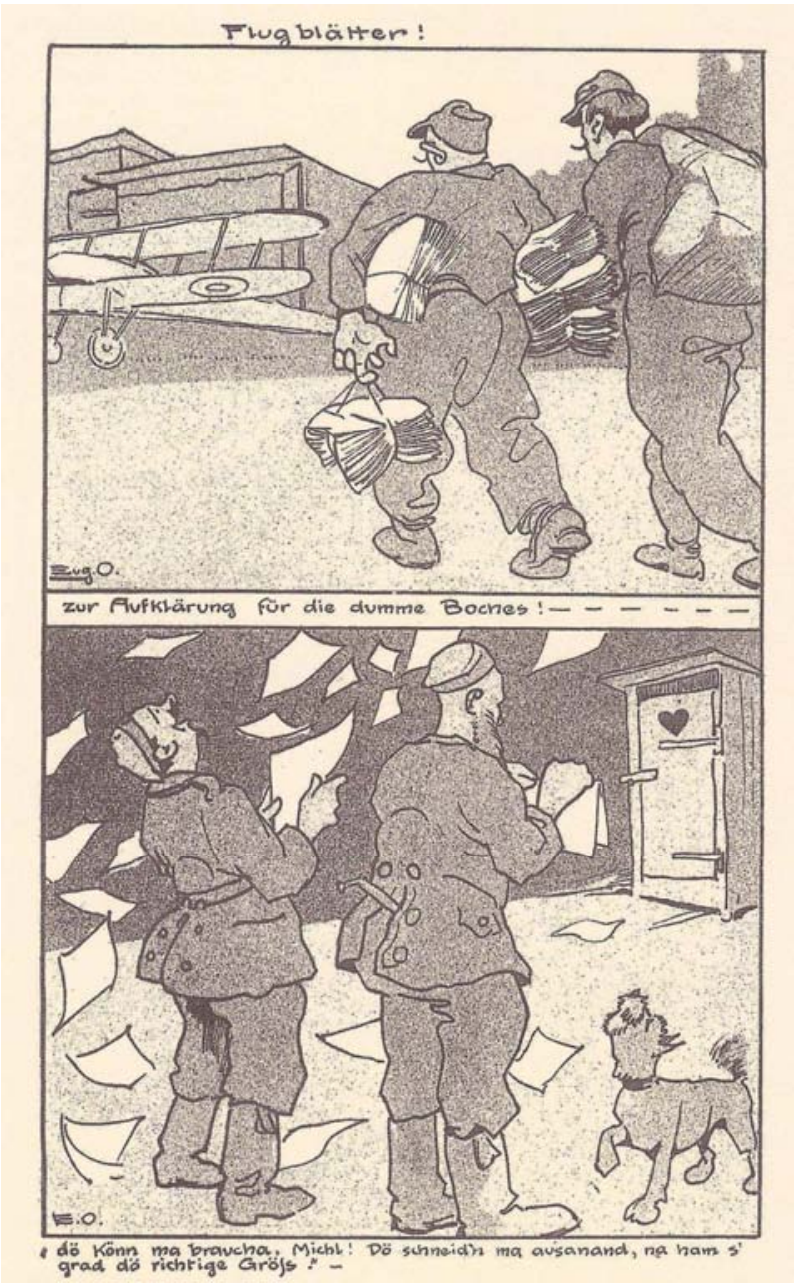

Abb. 8: Der Drahtverhau.

2) „Kriegszeitung der 4. Armee“: Kriegs-Struwwelpeter

(29.07.1917) [S.35] (Abb. 9). Der Zeichner Franz Breest hat auf dieser Beilage für die Kriegszeitung eine Parodie der „Geschichte vom bösen Friederich“ aus dem „Struwwelpeter“ von Heinrich Hoffmann (1845) verfasst, dessen Originaltext als Subtext dem Leser gegenwärtig ist: „Der Friederich, der Friederich, der war ein arger Wüterich!“" Friederich ist das ideale Objekt der „schwarzen Pädagogik“, auf das sich alle Erziehungsansätze des deutschen „Oberlehrers“ im 19. Jahrhundert 
(vergeblich) konzentrieren. Die - wie man heute sagen würde - ADHS-Symptome (Aufmerksamkeitsdefizit-Hyperaktivitätsstörung) des Kindes werden als Ausdruck einer kranken bis bösartigen Psyche gedeutet, die die gerechte Strafe eines Hundebisses „recht tief bis in das Blut hinein“ ereilt. Die Rolle des „Friederich“ übernimmt in Breests Zeichnung der gemeingefährliche Kosak „Nikolaus“, der „Petroleum trank gleich wie Bier“ und „zu Kleinholz machte das Klavier“.

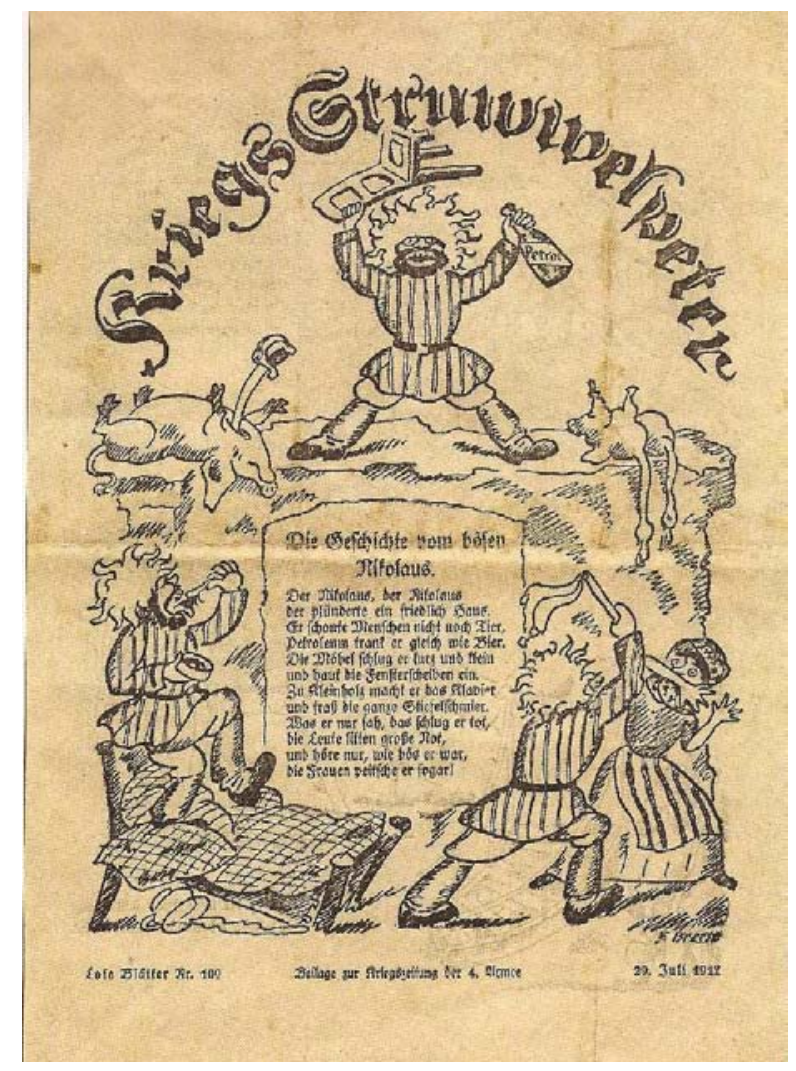

\section{Abb. 9: Der Kriegs-Struwwelpeter.}

Mit allen unmenschlichen Attributen ausgestattet - „er schonte Menschen nicht noch Tier" - stellt er eine Gefahr für die Zivilisation dar, die quasi aus Notwehr heraus unschädlich gemacht werden muss. Ein Gegner bar aller menschlichen Züge - „was er nur sah, das schlug er tot" - darf auch nicht auf eine humane Behandlung seitens der deutschen Soldaten hoffen. Die Bild-Text-Botschaft legitimiert im Gegenteil jede Gewaltanwendung. Nikolausens Kopfbedeckung bzw. Haartracht knüpft in Struwwelpeter-Manier Assoziationen an das Medusenhaupt, dessen Anblick die Gegner vor Angst zu Stein erstarren ließ. Der Schrecken er- 
reicht seinen - ironisch gebrochenen - Höhepunkt, wenn Nikolaus mit Schuhen und „Stiefelschmier" fressend durch das Bett tanzt und dabei „sogar" das Nachtgeschirr umwirft. Hinter der Fassade einer kindlichen Lehr-Lektüre offenbart sich der fanatische Wille zum entstellenden Blick auf den Gegner, der das eigene barbarische Handeln legitimieren soll.

3) „Der bayerische Landwehrmann“: Der Kreislauf

(1915/16) (Abb. 10). Wiggerl Greiner ist der Autor dieser Bildfolge, die den Kriterien der bande dessinée bereits sehr nahe kommt und die Monotonie sowie die katastrophalen hygienischen Zustände des Alltags im Schützengraben karikiert.

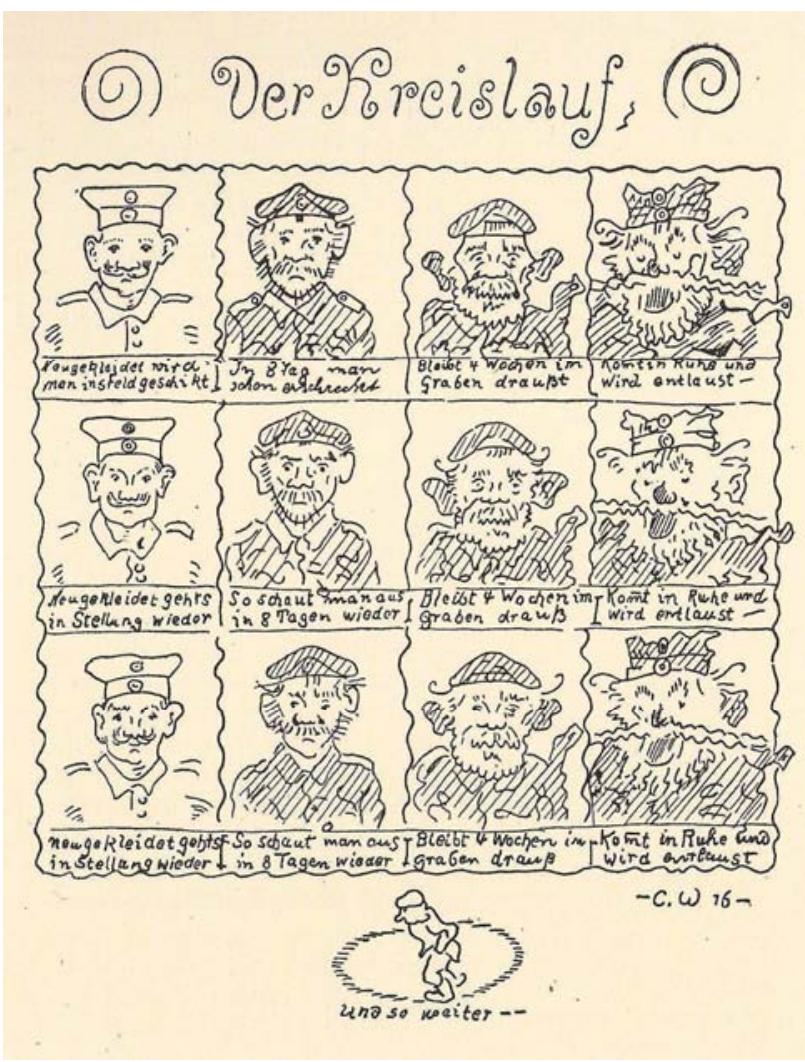

\section{Abb. 10: Der Kreislauf.}

Der vergebliche Kampf gegen Verlausung und körperlichen Verfall spiegelt symbolisch aber auch den inneren, psychischen Verfall der Soldaten wider, deren täglicher Überlebenskampf zu einer pervertierten „Normalität“ mutiert. Hinter dem ironischen Selbstporträt entdecken die Soldaten nicht nur ihre eigene Lebensrea- 
lität, sondern auch ihre reale Angst angesichts der Tatsache, dass dieser circulus diaboli keine Perspektive auf eine nahe Wende bietet. Auch die äußere Form - die Uniform nebst Dienstgradabzeichen - zerfällt innerhalb der Frist von vier Wochen und verliert ihre Kontenance bzw. Bedeutung: Im Schützengraben sind alle Soldaten gleich welchen Ranges Opfer. Die serielle Chronologie verläuft horizontal und vertikal. Je Zeile ist innerhalb des vierteiligen Zyklus' der Verfall erkennbar, aber es lässt sich auch eine Steigerung von Zeile zu Zeile erkennen. Der im Kreis auf seinen eigenen Spuren schlurfende Soldat - „und so weiter...“ - deutet eine unendliche Wiederholung dieser immer gleichen Episode an, deren Sinnhaftigkeit dadurch in Frage gestellt wird. Der Soldat ist einem Mechanismus ausgeliefert, den niemand mehr zu kontrollieren scheint. Sein sinnentleerter, stierer Blick steht im Kontrast zu den seltsamen Verzierungen im Titelschriftzug und Bildrahmen, die vielleicht auf die Befürchtung des Autors zurückzuführen sind, die Porträtzeichnungen könnten zu realistisch und damit zu frustrierend für die Zielgruppe dieses Bilderbogens gezeichnet sein. Tatsächlich ist hier die Abstumpfung gegenüber der Allgegenwärtigkeit der Verrohung und des Todes ablesbar:

\section{Soldatenbrief:}

„18. August 1916, Favreuil; Der Fahrer Renz, der ja gestern Nacht gefallen ist, wird morgen begraben. Den ganzen Leib hatte er voller Schrappnellkugeln. Ihr müsst Euch deswegen keine Angst machen, wenn's einen trifft, dann in Gottes Namen." ${ }^{14}$

4) „Liller Kriegsbilderbogen“ Nr. 21: Männer, Frauen und Kinder!

(16.02.1915) [S. 34] (Abb. 11). Es liegen kaum Hinweise auf Verbreitungsgrad und Auflagenhöhe dieser häufig laienhaft gefertigten Publikationen vor. Achim Schnurrer nennt für den mit logistischer Unterstützung der Heeresleitung produzierten „Liller Kriegsbilderbogen“ die Zahl von 85.000 Exemplaren ${ }^{15}$, was der Auflage einer mittleren deutschen Tageszeitung der Zeit entspricht („Vorwärts“ 150.000, „Der Wahre Jacob“ 163.000). Die meisten Feldzeitungen, die mit primitiven Druckvorrichtungen produziert werden, bleiben sicherlich weit darunter. Zahlreiche Blätter sprechen gezielt das eigene Regiment an, das im Durchschnitt 650 Männer umfasst. Eine „Armee-Zeitung“ hätte demgegenüber potenzielle 30.000 Leser ansprechen können (4. Armee an verschiedenen Kriegsschauplätzen in Flandern).

Der anonyme Zeichner dieses Comic-Strips spielt mit dem Motiv des furor teutonicus, der in seiner überspitzten, gewalttätigen Ausprägung auf diesen zehn Bildern wohl komisch wirken soll. Das Deutsche Reich sowie seine Kriegsgegner Belgien, Frankreich, Russland, Japan und England werden in allegorischen Figuren

14 Brief des Divisionsfahrers Otto Maute an seine Familie bei Bailingen, zit. n: Hirschfeld; Krumeich 2006: 97.

15 Schnurrer 2007: 35 . 
verkörpert. Dem uniformierten Japaner wird lediglich ein Bild gewidmet, wahrscheinlich, weil der asiatische Kriegsschauplatz um die deutsche Kolonie Kiautschou zu fern für die Soldaten an der Westfront liegt - aber auch im fernen Orient brüstet man sich mit dem negativen Image: „Nur ein Deutscher ist voll Niedertracht“. Die vier übrigen Nationen werden jeweils auf einem ersten Bild friedlich verharrend dargestellt, bevor sie auf dem folgenden Bild von einem deutschen Soldaten - scheinbar grundlos - attackiert werden: Dem belgischen Mädchen wird schmerzhaft die Nase umgedreht, der französische Soldat wird hinterrücks erstochen, der Russe ertränkt, der Engländer seiner Flotte beraubt.

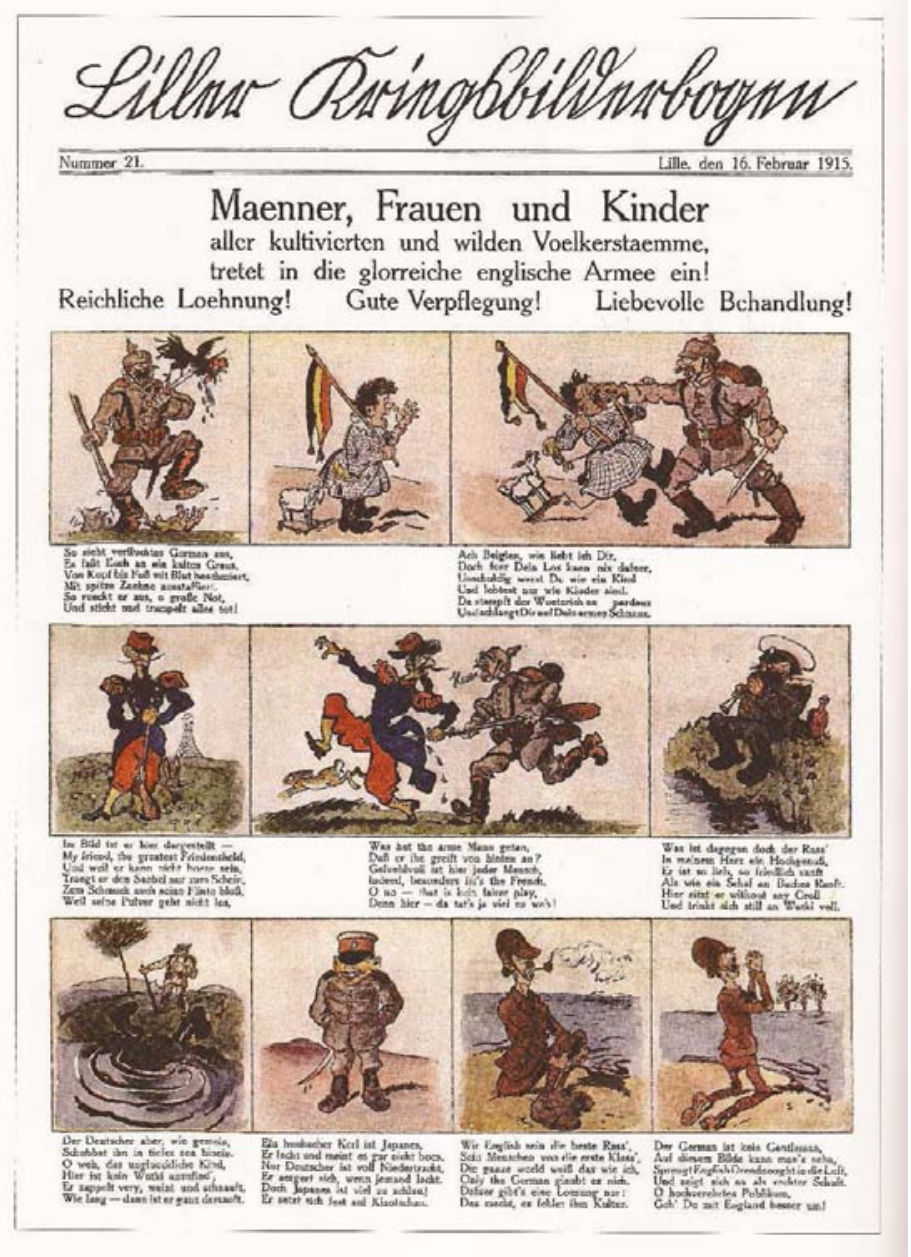

Abb. 11: Liller Kriegsbilderbogen. 
Die eigentliche Botschaft der Bilderreihe soll tatsächlich all diese Bilder und Klischees von ,angeblichen“ deutschen Kriegsverbrechen ad absurdum führen. Tatsächlich kursieren im Frühjahr 1915 beiderseits der Front Gerüchte über deutsche Greueltaten: „Dass Kindern ,die Hände abgehackt', Frauen ,Brüste abgeschnitten“, Nonnen geschändet würden, gehörte bald zum Repertoire derlei Horrorgeschichten. ${ }^{\text {،16 }}$

Die beiden irischen Historiker John Horne und Alan Kramer haben in ihrer Untersuchung „Deutsche Kriegsgreuel 1914“ (Horne; Kramer 2004) zwar nachgewiesen, dass Gerüchte über „abgehackte Kinderhände“ eine Erfindung der alliierten Propaganda waren, dass aber gleichwohl zahlreiche Kriegsverbrechen durch deutsche Truppen - besonders an der belgischen Zivilbevölkerung - zwischen August und Oktober 1914 begangen worden sind. Gerade im Frontabschnitt des „Liller Kriegsbilderbogens“ zwischen Leuwen und Dinant werden in dem besagten Zeitraum 6427 Zivilisten - auch Kinder und Frauen - Opfer von MassenerschieBungen im Kampf gegen angebliche Freischärler. Im Zuge dieser Propagandaschlacht stilisiert sich die deutsche Seite in diesem Comic zum Opfer einer Verleumdungskampagne und nutzt gleichzeitig ihre gespielte Empörung, um sich in einem Zuge von jeder Schuld freizusprechen. Nach der bewährten Formel „Negation gleich Affirmation“ verkörpert der deutsche Soldat also alle Tugenden, die auf den Zeichnungen in ihren negierten Versionen erscheinen: Er ist der „ritterliche“ Patriot, der mit offenem Visier kämpft und „natürlich“ die Zivilbevölkerung verschont. Die eigenen Truppen sollen in ihrer Überzeugung gestärkt werden, in einer Welt von heuchlerischen Feinden mit fairen Mitteln für die richtige Sache zu kämpfen. Die Tatsache allerdings, dass diese Gerüchte überhaupt in einer ,halbamtlichen“ Frontzeitung angesprochen werden, zeugt davon, dass es Unruhe in den eigenen Reihen gegeben hat, die hier mit Polemik bekämpft werden soll.

\section{Bunte Kriegsbilderbogen von Walter Trier (1890-1951)}

Eckart Sackmann hat für die Wissenschaftsreihe „Deutsche Comicforschung“ (Sackmann 2008) das weltkriegsspezifische Comic-Genre der Kriegsbilderbogen wiederentdeckt. Der bekannteste Titel dieses Genres ist der „Bunte Kriegsbilderbogen“, der in den Jahren 1914 und 1915 im Berliner „Verlag der Vereinigung der Kunstfreunde Otto Troitzsch“ erscheint. Wie die meisten satirischen Publikationen - „Simplizissimus“, „Wahrer Jacob“, „Lustige Blätter“ usw. - widmen sich auch die Kriegsbilderbogen seit dem Kriegsausbruch im Sommer 1914 ganz der chauvinistischen Kriegsbegeisterung. Der ideologische Mainstream setzt sich auch bei den anspruchsvollsten Zeichnern auf ganzer Linie durch: „Niemand mochte sich offenbar dem nationalen Trend widersetzen; es sind bisher keine Beispiele von 
comic-künstlerischem Widerstand bekannt. [...] Während die Bogen mit den Bildergeschichten künstlerisch zum Teil hervorragend sind, werden die Inhalte getragen von plattestem Hurra-Patriotismus. ${ }^{\text {(17 }}{ }^{17}$ Die Comic-Strips umfassen jeweils fünf bis zehn farbige Bilder, die in drei Zeilen angeordnet sind. Der Farblichtdruck (Troitzschotypie) beschränkt sich auf vier Grundfarben in konstanter Farbintensität. Die Bildgeschichten folgen einem zusammenhängenden Erzählstrang oder lassen sich noch einmal in zwei oder drei Einzelepisoden unterteilen, die aber in einem thematisch übergreifenden Kontext zueinander stehen.

Der „Verlag der Vereinigung der Kunstfreunde Otto Troitzsch“ spezialisiert sich auf die Massenproduktion von eher kitschigen Kunstdrucken (Schutzengel, keusche Liebesszenen, Landschaftsidyllen), die Einzug in die Wohnzimmer der kleinbürgerlichen Mittelschichten finden. Dem politischen und moralischen Trend der Zeit folgend erweitert Troitzsch seine Angebotspalette mühelos um das gewaltund vaterlandsverherrlichende Motiv. Es lässt sich nur spekulieren, in welcher Weise der Kriegsbilderbogen seinen ideologischen Appell in die Privatsphären der deutschen Familie transportiert, oder vielleicht als „Wegwerfprodukt“ - bei einem Preis von 10 Pfennig je Exemplar - nach einmaligem Lesen entsorgt wird.

\section{1) K.F.A.C. (Kaiserliches Freiwilliges Automobil Corps)}

(Nr. 32, 1914) (Abb. 12). Walter Trier gehört neben Paul Wendling, Leo Leipziger, Ludwig Kainer u.a. während des Ersten Weltkrieges zu den regelmäßigen Mitarbeitern der „Bunten Kriegsbilderbogen“. 1890 in Prag geboren zieht es ihn zunächst 1906 nach München, bevor er ab 1910 in Berlin für den Verleger Hermann Ullstein arbeitet. Rasch wird er dort zu einem gefragten Werbe- und Buchillustrator heute sind insbesondere seine Illustrationen für die Werke Erich Kästners (ab 1929) noch in Erinnerung. Gleichzeitig erscheinen seine Zeichnungen und Karikaturen im „Simplicissimus", in „Jugend“, und in den „Lustigen Blättern“ von Otto Eysler. Im Duktus folgt er während des Ersten Weltkrieges dem herrschenden patriotischen Pathos, zeigt in seinem zeichnerischen Stil aber bereits einen kreativen, eigenen Ansatz. Antje Neuner-Warthorst ordnet seinen Stil als Produkt des „klimatischen Umfeldes [...] im Redaktionskollegium von Eyslers Lustigen Blättern“'،18 ein, zu dem neben Paul Simmel auch Heinrich Zille zählt. Bis 1946 veröffentlicht Trier rund 350 Bildergeschichten und Einzelbildwitze. Als Gattungsbegriff für den Comic-Strip wählt er die Bezeichnungen „Zeichnerscherz“ bzw. - als jiddische Variante - „Meschuggenes“ und verweist damit bereits auf seine jüdische Herkunft, die ihn 1936 dazu zwingen wird, mit seiner Familie nach England zu emigrieren.

Bei dieser sechsteiligen Bildfolge fallen sogleich die progressiven Stilmittel Triers ins Auge: Der monochrome, gelbe Hintergrund für die Aktionsmittelpunkte der Bilder 3 und 5 erinnert an den berühmten Umschlagentwurf für „Emil und die

17 Sackmann 2007: 45.

18 Neuner-Warthorst 2007: 49. Siehe auch: http://www.walter-trier.de/Lebensdaten.htm. 
Detektive“ (1929). Speed-lines und stilisierte Wölkchen, die zu diesem Zeitpunkt bei Zille noch keine Rolle spielen, verleihen den Bewegungen ihre Dynamik. Inhaltlich soll die Bildgeschichte die technische Überlegenheit der deutschen Truppen gegenüber den Franzosen herausstreichen: Der motorisierte deutsche Offizier besiegt alleine, als typisch unbesiegbarer Comic-Held, eine ganze Armee von französischen Zuaven und Dragonern.
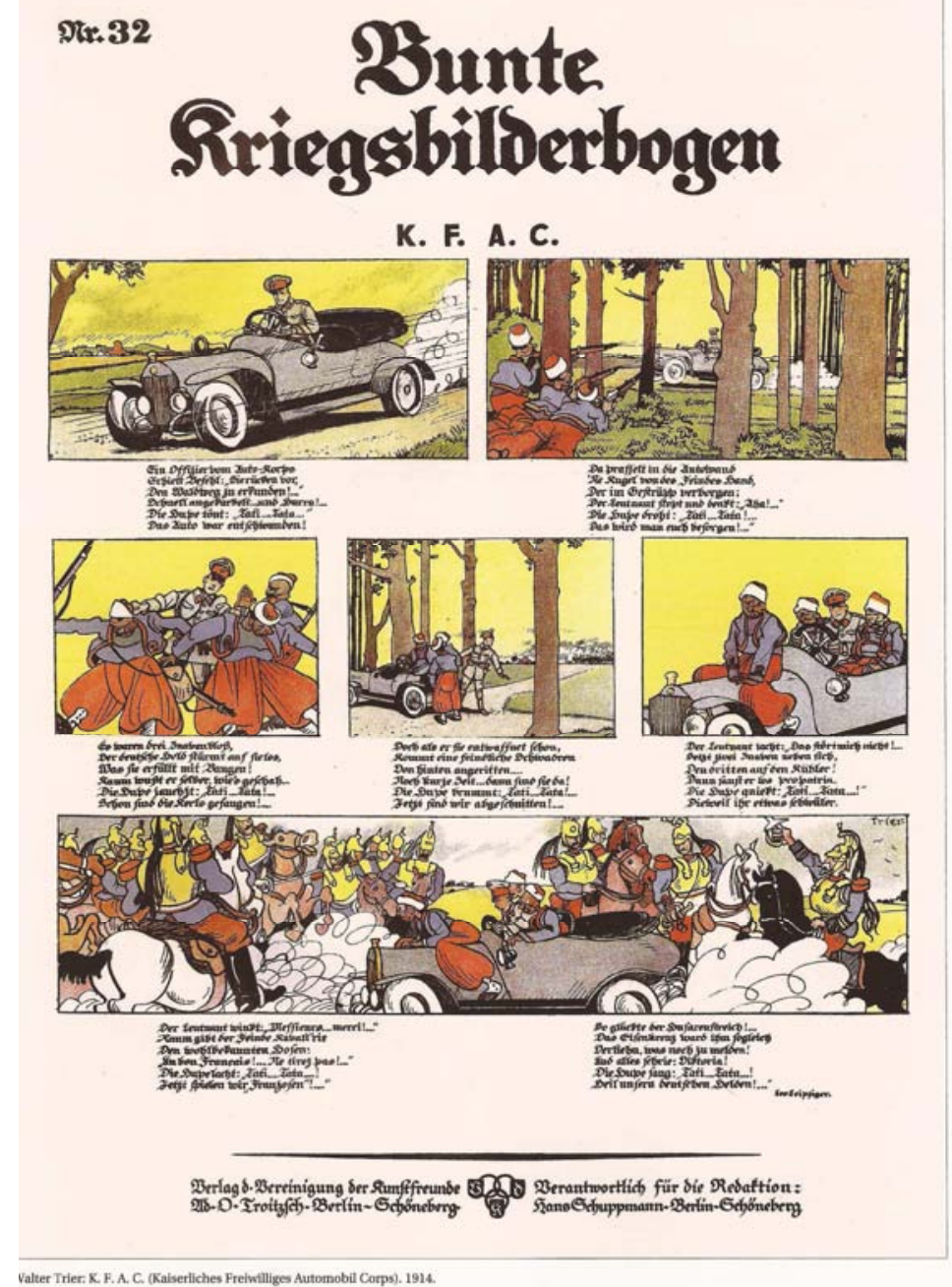

Abb. 12: Walter Trier, Kriegsbilderbogen K.F.A.C.

Die französischen Soldaten wirken in ihren Uniformen aus dem Krieg von 1870 lächerlich rückständig gegenüber der modern ausgestatteten deutschen Armee. Mit 
typisch deutschen Tugenden - Mut und Kaltblütigkeit - ausgestattet, soll der Endsieg als zwangsläufige Logik erscheinen. Bar aller Realitätsnähe erzählt Trier eine kleine Action-Episode, bei der die Franzosen am Ende die Verlierer sind, ohne aber diese propagandistisch über die Maßen zu diffamieren. Durch die Darstellung der Kolonialtruppen gewinnt die Geschichte allerdings eine rassistische Komponente, die den Wert der französischen Zivilisation insgesamt in Frage stellen soll.

\section{2) Der Kosake Wladimir}

(Nr. 2, 1914). Etwas weniger dynamisch, aber wiederum mit dem gelben Bildhintergrund erscheinen die sieben (hier nicht abgebildeten) Zeichnungen, die den russischen Gegner an der Ostfront porträtieren. Das ungepflegte Konterfei des „Kosaken Wladimir“ karikiert den Gegner sehr detailliert in entstellender Weise. Um die Überlegenheit der deutschen Truppen herauszustreichen, zitiert Trier das Motiv des „Ritters von der traurigen Gestalt“. Das erste Bild stellt den russischen Soldaten als lächerliche Gestalt in der Art eines Don Quichotte dar. Sinnbildlich beschreibt die Darstellung den schlechten Zustand von Ausrüstung und Verpflegung der russischen Armee, deren Kampfgeist völlig erloschen scheint. In der Kaviar-Dose befindet sich nur Sand. Sogar das Pferd Olga sehnt sich nach der deutschen Gefangenschaft: „Futter hofft auch sie zu kriegen, wie ihr Reiter Wladimir.“ Die realistische Zeichnung „unsrer schneidigen Ulanen“, die dem freiwillig gefangenen Kosaken eine bessere Verpflegung bieten, als er sie jemals zuvor genossen habe, suggeriert eine totale deutsche Überlegenheit. Die Diffamierung gipfelt im vorletzten Bild darin, dass der russische Soldat in moralisch verwerflicher Unmanier seine deutschen Helfer bestiehlt: „Zu dem Nachtisch aber schmauste, Wladimir, weil er sie mauste, eine Kerze Stearin.“ Aus Dummheit und aus mangelnder Kultiviertheit heraus isst der Russe die Stearinkerze, weil er wohl nicht weiß, wozu diese eigentlich dient (Stearin wird aus pflanzlichen und tierischen Ölen und Fetten gewonnen, ist essbar und dient primär der Kerzen-Herstellung). Auch an der Ostfront scheint der Endsieg einer zwangsläufigen Logik zu entspringen. Die materielle Überlegenheit der „deutschen Hochkultur" gegenüber den „barbarischen Russen“ ist überdeutlich.

\section{3) Lustige Blätter}

Nr. 3, 1915 (zit. n. „Rire“, 09-06-1939): La Triplice est dissoute (Abb. 13). Es folgt nun ein Kapitel tragischer Rezeptionsgeschichte des Werkes von Walter Trier: Die Karikatur stammt zwar ursprünglich aus den Berliner „Lustigen Blättern“, ist hier aber als Reproduktion aus dem französischen journal bumoristique „Le Rire“ vom 9. Juni 1939 zu sehen. Auf der Titelseite eine Karikatur von Franz Jüttner (18651925): „,... und jetzt werden wir dem Bürschchen eine Lektion erteilen!“ bezieht sich auf den Eintritt Italiens 1915 auf der Seite der Entente unter der Führung des italienischen Königs Viktor Emmanuel III. 
Im Mai 1915 widmen sich zahlreiche Karikaturisten ausgiebig dem diffamierenden Porträt angeblicher italienischer Untreue gegenüber dem Dreibund-Vertrag zwischen Österreich-Ungarn, Deutschem Reich und Italien aus dem Jahre 1882 (Abb. 13). Im Original lautet die sprachspielerische Bildunterschrift: „Der Dreibund ist aufgelöst. Es bleibt ein Zweibund ... und ein Vagabund!“ Der realistischen Darstellung des österreichischen und deutschen Soldaten in solidarischer Pose steht die Karikatur des Bersagliere gegenüber, der kleiner gewachsen, mit hämischem Grinsen auf die ehemaligen Verbündeten zielt. Die Chianti-Flasche an seiner Seite soll Disziplinlosigkeit suggerieren. Ein häufig wiederkehrendes grafisches Mittel bei Trier ist das Spiel mit dem Bildrahmen. Der in das Nachbarbild hineinragende Gewehrlauf unterstreicht die Gefährlichkeit des scheinbar unvermittelt angreifenden Italieners.

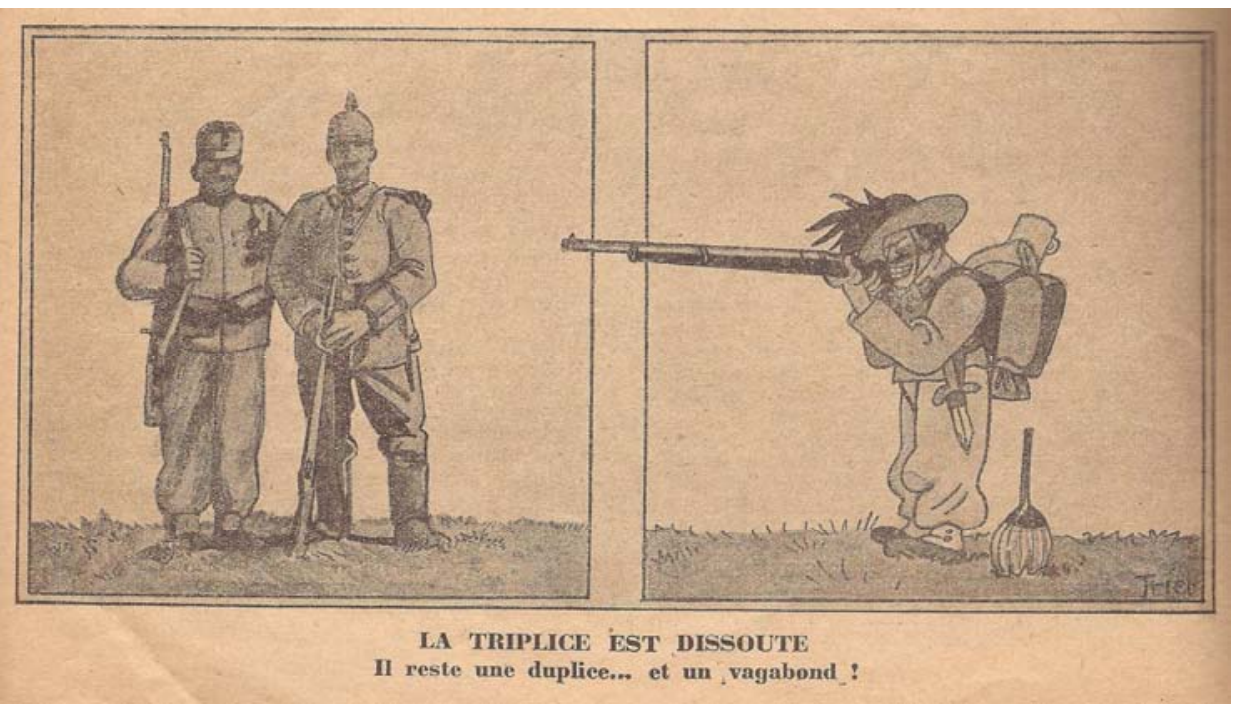

Abb. 13: Walter Trier, La triplice est dissoute.

Der Grund für die Reproduktion einer Auswahl von Karikaturen aus den deutschen „Lustigen Blättern“ zum Thema „Les Italiens vus par les Allemands“ ist der soeben beschlossene Stahlpakt zwischen Hitler und Mussolini (22. Mai 1939). Die Freunde von heute sind die Gegner von gestern - oder frei nach Talleyrand: Die Wahrheit ist immer eine Frage des Zeitpunktes. Deutsche Karikaturisten werden zwanzig Jahre später als Zeugen angeblicher italienischer Wankelmütigkeit zitiert. Obendrein wird die Stabilität des zum „Stahlpakt“ stilisierten Bündnisses der beiden faschistischen Regime in Frage gestellt: Wo gerade noch die charakterliche Schwäche und Unzuverlässigkeit der Italiener in den deutschen Medien angeprangert wurde, wird nun plötzlich eine unzerbrechliche Freundschaft beschworen. Ohne die Originalkarikaturen zu modifizieren und lediglich mit 
französischen Übersetzungen ausgestattet sprechen die 25 Bilder des „Numéro spécial du Rire" für sich, indem sie die geheuchelte deutsch-italienische Propaganda entlarven. Im Vorwort heißt es lediglich: „Il nous est particulièrement agréable de relire les injures, les calomnies, qui furent jetées, à cette époque, sur nos voisins du sud-est par leurs amis d'aujourd'hui. L'Allemagne, à la nouvelle que l'Italie se rangeait du côté de l'Entente anglo-française, entra dans une rage hystérique dont les dessins réunis ici donnent une excellente idée." Ein Hinweis auf die sehr aufmerksame gegenseitige Rezeption der deutsch-französischen Karikaturistenszene, die in der Feststellung gipfelt: „Il n'y eut pas, en 1915, un seul caricaturiste allemand, pas un seul journaliste, pas un seul écrivain, pas un seul orateur public qui sut garder quelque mesure." In der Tat verlieren Künstler und Politiker auf allen Seiten während des Ersten Weltkrieges jedes menschliche Maß Walter Trier ist daher sicherlich nicht der einzige und auch nicht der fanatischste Karikaturist auf deutscher Seite. Fatal, geradezu tragisch ist es aber, dass ausgerechnet der Jude Trier 1939 in Frankreich indirekt als Agent des faschistischen Regimes Hitlers zitiert wird. Denn zu diesem Zeitpunkt lebt er mit seiner Familie bereits seit vier Jahren in London und publiziert Zeichnungen mit einer ganz anderen Aussage (Abb. 14).

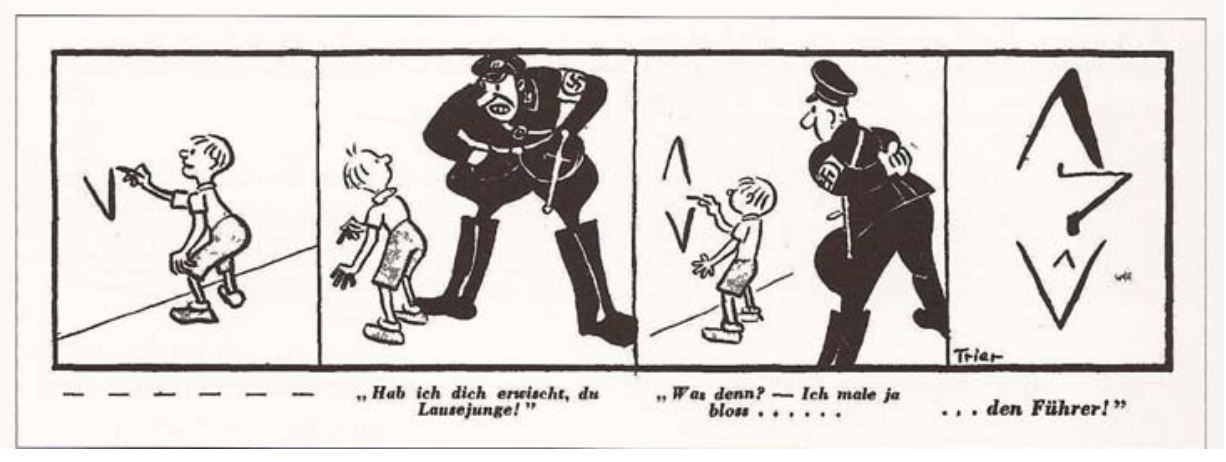

Abb. 14: Londoner Zeichnung von Walter Trier.

Von 1941 bis Kriegsende werden seine Anti-Nazi-Cartoons in der vom englischen Informations-Ministerium publizierten Zeitschrift „Die Zeitung“ regelmäßig veröffentlicht. Trier wird nach dem Krieg britischer Staatsbürger und reist 1949 zu seinen Töchtern nach Kanada aus, wo er 1951 in Toronto stirbt.

\section{Schluss}

Die hier vorgestellten Bild- und Textmaterialien zeigen die Vielschichtigkeit der Einsatzmöglichkeiten von populärliterarischen Medien im DaF-Unterricht. Erstens illustrieren sie besser als Sachtexte und Fachvorträge einzelne gesellschaftliche und 
politische Aspekte einer Epoche. Massenpsychologische Stimmungen und Befindlichkeiten in der Bevölkerung - als „Volkes Stimme“ verdichtet - finden in Bildund Textgattungen, die für einen Massenkonsum gedacht sind, einen authentischeren Widerhall. Zweitens bieten sie besonders motivierende Sprechanlässe - jedes Bild stellt sich zunächst als Suchbild und Rätsel dar, das dem Betrachter bei einer Bildbeschreibung eine gewisse Interpretationsfreiheit lässt und zunächst zum freien Assoziieren einlädt. Visuelle Eindrücke bieten einen direkten Einstieg auch in abstrakte Themenfelder. Drittens bietet das Medium gerade für den Aspekt der interkulturellen Horizont-Erweiterung viele Anknüpfungspunkte bei der Aufarbeitung deutsch-französischer Erinnerungsorte aus dem Ersten Weltkrieg. Um im Medium zu bleiben, würde sich eine Vertiefung des Themas über eine vergleichende Betrachtung französischer Karikaturen und Comic-Strips anbieten. Hier müsste unbedingt auch auf die Erinnerungsarbeit von Jacques Tardi zurückgegriffen werden mit seinen epochalen Werken: „C'était la guerre des tranchées“ (1993), „Le der des ders“ (1996), „Varlot soldat“ (1999) und den Illustrationen zu Célines „Voyage au bout de la nuit" (1988). ${ }^{19}$ Schließlich stellt das Bildmedium selbst bereits ein Dokument deutscher Kultur dar, das gerade bei Künstlern wie Zille und Trier eine eingehendere Würdigung verdient. Gerade für die tendenziell BDbegeisterteren französischen Deutschlernenden wäre dies sicherlich die Entdeckung eines „starken Stückes Deutschlands“.

\section{Quellen}

Rigolboche, Nr. 7, 20.04.1915 (Bibliothèque Nationale, Cote LC6-144).

Numéro spécial du „Rire“, Nr. 1037, 09.06.1939.

Hansi, alias Jean-Jacques Waltz (1918): Le Paradis Tricolore. Paris: Herscher.

Ostwald, Hans (Hrsg.) (1942): Zille's Hausschatz,. Berlin: Paul Franke Verlag.

Zille, Heinrich (1915/16): Vadding in Frankreich (2 Bde.). Berlin: Verlag „Lustige Blätter“.

\section{Literatur}

François, Etienne; Schulze, Hagen (Hrsg.) (2001): Deutsche Erinnerungsorte. München: Beck.

Hirschfeld, Gerhard; Krumeich, Gerd (Hrsg.) (2006): Die Deutschen an der Somme 1914-1918. Essen: Klartext.

${ }^{19}$ Vgl. Sistig 2002. 
Horne, John; Kramer, Alan (2004): German Atrocities 1914. New Haven: Yale University Press.

Isnenghi, Mario (Hrsg.) (1996/1997): I luoghi della memoria. Roma: Laterza.

Kohlrausch, Martin (2010): Das Reich bin ich - Selbstherrlich, dilettantisch, modern: Wilhelm II. In: ZEIT Geschichte, Nr. 4, 2010, 60-63.

Lemmermann, Heinz (1984): Kriegserziehung im Kaiserreich. Bremen: Eres Edition.

Neuner-Warthorst, Antje (2007): ,Meschuggenes` von Walter Trier. In: Sackmann (Hrsg.) (2008), 48-61.

Nipperdey, Thomas (1998): Deutsche Geschichte. München: Beck.

Nora, Pierre (Hrsg.) (1984): Les lieux de mémoire Bd. 1. Paris: Gallimard.

Nora, Pierre (Hrsg.) (1986): Les lieux de mémoire Bd. 2. Paris: Gallimard.

Nora, Pierre (Hrsg.) (1992): Les lieux de mémoire Bd. 3. Paris: Gallimard.

Sackmann, Eckart (2007): Bunte Kriegsbilderbogen. In: Ders. (Hrsg.) (2008), 4447.

Sackmann, Eckart (Hrsg.) (2008): Deutsche Comicforschung 2008. Hildesheim: Comicplus+.

Schnurrer, Achim (2007): Aus der Bildermappe des Meldereiters. In: Sackmann (Hrsg.) (2008), 34-43.

Sistig, Joachim (2002): Invasion aus der Vergangenheit. Das Deutschlandbild in frankophonen Bandes Dessinées. Frankfurt/Main: Peter Lang.

Tubergue, Jean-Pierre; Charpentier, André (2007): La plume au fusil: 1914-1918. Les journaux des tranchées. Paris: Editions Italiques.

Ullrich, Volker (2004): Krieg der Worte, Kampf der Bilder. In: Die Zeit, 24.06.2004, 41 .

Wehler, Hans-Ulrich (1998): Der erste totale Krieg. In: Die Zeit, 20.08.1998, 35. 



\title{
Plakate im DaF-Unterricht
}

\author{
Marc Hieronimus
}

\section{Einleitung}

Seit dem ausgehenden neunzehnten Jahrhundert werden deutsche Großstädte mit Plakaten tapeziert. Ihre Zahl dürfte über die Jahre erheblich gestiegen sein, über die Qualität lässt sich streiten: „Plakate sind in der Politik wie in der MarkenartikelWerbung nur noch Sekundanten. So erklärt sich die Armut ihrer Argumentation und das Bemühen um ästhetischen Schick“" hieß es schon vor über dreißig Jahren (Arnold 1977: keine Seitenangabe). Ob man angesichts ihrer Allgegenwart wirklich von einer Zweitrangigkeit sprechen kann, sei dahingestellt; sicher ist, dass Plakate weit mehr als nur Dekor, nämlich Ausdruck ihrer Zeit sind. Werbebilder und -botschaften - und das heißt auch politische - können „,hochsensible Quellen mit geradezu prismatischen Eigenschaften" darstellen, und zwar selbst wenig erfolgreiche. „Der Idealfall freilich scheint die durch Werbung zustande gekommene sinnfällige oder gar sinnstiftende Abbreviatur gesamtgesellschaftlichen Selbstverständnisses als griffige und formelhafte Bewußtseinsmünze." (Gries; Ilgen; Schindelbeck 1995a: 17f.).

Gemessen an ihrer Verbreitung und Bedeutung erfahren Plakate, abgesehen von einigen semiotischen Studien und populärwissenschaftlichen Motiv- und Firmengeschichten, in der Geschichtswissenschaft allerdings nur wenig Aufmerksamkeit. ${ }^{1}$ Das mag auf internationaler Ebene nicht zuletzt an einer Begriffsverwirrung liegen: Auf Englisch heißen die Plakate „posters“, sind aber keine; denn anders als

${ }^{1}$ Einen Überblick gibt Seidensticker 1995: $5 f$. 
die Poster im deutschen Sinne sind Plakate auf Außengebrauch hin ausgelegt, und im Gegensatz zum Kunstplakat, das auch unter den englischen Poster-Begriff fällt, im Deutschen aber meist mit (Original- oder Repro-) „Druck“ bezeichnet wird, sind Plakate, wenn auch oft von Künstlern gestaltet, fast ausschließlich kommerzieller oder politischer Natur. ${ }^{2}$

So definiert wird die Werbe- und Druckgeschichte zur Vorgeschichte des Plakats. Hanns Buchli (1962) geht sicher zu weit, wenn er, gestützt auf Beispiele aus Uruk und anderen alttestamentarischen Grabungsstätten, von „,6000 Jahren Werbung“" spricht; selbst nach einer weiten Definition wie der seinen fallen bloße Hinweisschilder doch nicht unter die Kategorie. Wichtig ist sein Hinweis auf die gleichen Wurzeln von Wirtschaftswerbung und politischer Propaganda. Er spricht von zwei Erscheinungsformen der Werbung. Wirtschaftswerbung und politische oder weltanschauliche Propaganda (wie etwa die Mitgliederwerbung [sic!] für religiöse Strömungen) bedienten sich gleicher Mittel, und das Objekt sei in jedem Falle der Mensch (Buchli 1962: 42). So kommt er zu folgenden Begriffsbestimmungen: „Werbung ist eine Beeinflussung des Menschen, die ihn veranlaßt, etwas freiwillig zu tun“, nämlich „,sich freiwillig eine Überzeugung anzueignen und sie als wahr anzuerkennen" im Falle der politischen Werbung oder Propaganda, oder „freiwillig ein Geschäft abzuschließen“ in dem der Wirtschaftswerbung (Buchli 1962: 64). ${ }^{3}$

\subsection{Druckgeschichte}

Die Geschichte der Druckgrafik als Vorbedingung für die Entstehung des Plakats beginnt im Spätmittelalter. Auf das Jahr 1390 ist die älteste Urkunde über gewerbliche Papierherstellung in Deutschland datiert; erst als der Nürnberger Großkaufmann Ulrich Stromer und seine Nachfolger und Konkurrenten massenhaft Papier herstellten, konnte sich der Buch- und Bilddruck zunächst mit unbeweglichen, seit Gutenberg dann mit beweglichen Lettern fortentwickeln. Um 1440 kommen Kupferstiche auf, von denen man bis zu 2000 Abzüge machen kann; der gröbere Holzschnitt lässt sich beinah unbegrenzt wiederverwenden. Plötzlich gibt es also käufliche Bilder. „Dass von einem einzigen Original, einem Druckrelief, eine Vielzahl von Abzügen genommen werden konnte, dürfte im frühen 15.Jh. so ähnlich bestaunt worden sein wie die wundersame Brotvermehrung. [...] Solche wundersame Bildvermehrung bringt einen sozialen wie psychologischen Umschwung mit sich.

\footnotetext{
2 Warnungen und Hinweise werden in der Regel auf Schildern kommuniziert, und auch „ungeklebte“ Plakatsatire etwa in der Zeitschrift „Titanic“ fällt nicht unter den Begriff.

${ }^{3}$ Der Zusammenhang war früher geläufiger: Der Werbegrafiker Julius Klinger hat das Titelbild einer Zeitschrift mit Namen „Propganda“ gestaltet; das von Robert Exner herausgegebene Blatt zeigte laut Eigenwerbung „den Weg zum geschäftlichen Erfolg“. Ein anderes Beispiel: Hans Domizlaff, Berater von Reemtsma, Siemens und der Deutschen Grammophon und vielleicht der „Urfaust“ der deutschen Werbung, konnte „keinen Unterschied darin erblicken, ob [er] ein Wirtschafts-Unternehmen berate oder eine Staatsidee“ und brüstete sich damit, Goebbels habe sein Buch namens „Die Propagandamittel der Staatsidee“ auswendig gelernt, vgl. Schindelbeck 1992: 23f.
} 
[...] Wer beispielsweise den Hl. Christopherus nicht mehr nur an einer bestimmten Kirchenwand um Fürbitte anrufen muss, ihn stattdessen als gedrucktes Bild beliebig oft zur Hand und in den Blick nehmen kann, dessen Einstellung zu Seelenheil und Kunst wird sich grundlegend verändern." (Rebel 22009: 14f.). Um 1505 gibt es in Italien erste Beispiele für Reproduktionsgrafik (statt Originalgrafik). Dürer und Raffael gelten als die ersten reproduzierten Meister der Kunstgeschichte.

Über dreihundert Jahre blieben Kunstdrucke einem wohlhabenden Publikum vorbehalten. Litfaßsäulen, Anschlagkästen und „Sandwichmänner“ als öffentliche Bildträger kamen erst Mitte des neunzehnten Jahrhunderts auf, denn „[e]rst seit 1844, als Holzschliff als Rohmaterial für die Papierherstellung eingeführt wurde, konnte Papier preiswert und in großen Mengen hergestellt werden. " (Ingenkamp 1996: 164; vgl. auch Buchli 1966: 223), und noch ein wenig später erlaubte die Entwicklung des Rasterdrucks durch Georg Meisenbach die massenhafte Reproduktion jedes beliebigen Bildmotivs. Nun erst entsteht das Plakat als ,großformatiges, auf demonstrative Wirkung angelegtes Druckerzeugnis (lat. Placatus ,geebnet, geglättet'). [...] Technisch und wahrnehmungspsychologisch ist das Medium Plakat an die Erfindung der Lithografie geknüpft: an deren beschleunigte, auflagenstarke, kostengünstige und aktualitätsbegierige Reproduktionsleistung, aber auch an deren künstlerisch experimentelle Formkraft. Bei Einbeziehung fotomechanischer Komponenten wird das Plakat dann im System der industriellen Druckgrafik, also etwa ab 1890, ein bevorzugter Aufmerksamkeitsfänger und Werbeträger für politische Propaganda und kommerzielle Reklame." (Rebel 22009: 245).

Nun enstehen z.B. die heute noch geschätzten Kunst-/Werbeplakate Chérets und Toulouse-Lautrecs. Der Kunsthistoriker Ernst Rebel spricht in diesem Zusammenhang von einer (zweiten) „Transmedialisierung“: „Wie um 1500 mit der erreichten Gleichberechtigung von Malerei und Druckgrafik eine erste Transmedialisierung stattgefunden hat, so nun um 1900 mit erreichter Gleichberechtigung von künstlerischem und industrialisiert-öffentlichem Bild der gebrauchsgrafischen Medien eine zweite." (Rebel 22009: 126). ${ }^{4}$ Von den 1890er Jahren bis zur Wiemarer, vielleicht sogar Bonner Republik sollten Plakate ein bedeutendes Medium künstlerischer Entfaltung sein; es wird sich zeigen, dass die Grafiker und Werbefachleute wenig Bedenken hatten, ihre Kunst in den Dienst von Kommerz und Propaganda zu stellen.

\section{Werbeplakate}

Das Phänomen Werbung wird oft unterschätzt. „In den USA hat man schon 1968 in einer Untersuchung festgestellt, daß rund 1600 Werbeimpulse täglich auf den Verbraucher eindringen. Und das waren schon seinerzeit rund 500\% mehr als 20

${ }^{4} \mathrm{Zu}$ den Werken der (Kunst)Druckgrafik vgl. Rebel 2010. 
Jahre zuvor.“ (Schönert 1988: 46). Eine Fachindustrie von Grafikern, Psychologen, Soziologen, Lobbyisten, Marketingstrategen und anderen Experten arbeitet seit Jahrzehnten beharrlich an der stetigen Ausweitung der werblichen Beeinflussung des Bürgers durch Vermehrung der Träger und Kanäle und durch Verfeinerung der Mittel, oder positiv ausgedrückt: „Die Hauptaufgabe der modernen Werbung: sorgfältig und bedacht die individuelle Persönlichkeit des Konsumenten und seiner Bezugsgruppen aufzusuchen und anzusprechen." (Huber 1990: 129). Das wichtigste Mittel sind dabei die Bilder, denn sie erregen schneller größere und längere sowie emotionalere Aufmerksamkeit und transportieren ihre Botschaften subtiler als Texte. Dem entsprechend werden Werbebotschaften nach Einschätzung eines Werbefachmanns auch nur zu fünf Prozent bewusst wahrgenommen, zu 95 Prozent aber unbewusst emotional (Huber 1990: 15). ${ }^{5}$ Dem wird im Unterricht wenig entgegengesetzt. „Unsere Gesellschaft vermittelt primär Kulturtechniken im Umgang mit dem sprachlichen Symbolsystem - Lesen wie Schreiben - und vernachlässigt sträflich die visual literacy." (Schierl 2005: 309). ${ }^{6}$

Dabei ist die Erkenntnis, in einem visuellen Zeitalter zu leben, umgeben von halbverstandenen, aber dennoch wirksamen Zeichen, nicht neu. Schon zu Beginn der 1970er Jahre gab es durchaus Bestrebungen, den klassischen, vermeintlich ideologisch aufgeladenen Kunstunterricht durch das Fach „Visuelle Kommunikation“ zu ersetzen, in dem es zum Beispiel darum gegangen wäre, „Gebrauchsgegenstände wie: Kleider, Schränke, Parks, Flugzeuge, Autos, Zeitschriften, Gemälde, Fotos, Reklamematerial, Dias, Filme usw. als Träger von visueller Information (= vI) und als visuelle Phänomene ( $\mathrm{vPh})$ wahrnehmen und begreifen [zu] lernen“, oder das „Angebot an visueller Kommunikation im Bereich des Freizeitverhaltens als Angebot von Waren interpretieren [zu] lernen“. Insbesondere sollte es um eine „Sensibilisierung für die Möglichkeit, über vI manipuliert zu werden“ gehen und darum, die „Abhängigkeit der Produktion von vI von ökonomischen Bedingungen erkennen“" zu lernen. (Hartwig 1971: 339).

Kunsterziehung und Kunstunterricht als Erziehung durch bzw. zur Kunst wurde damals, bei aller heute vermeintlich obsoleten Rhetorik, völlig zu Recht als ungenügend kritisiert:

„a) Bildende Kunst ist seit langem, aber erst recht heute nicht mehr denn ein Teilbereich optischer Kultur; andere Bereiche optischer Kultur (Fotografie, Film, Fernsehen) haben ihn schon rein quantitativ weit überflügelt. b) Die quantitative Dominanz der sog. Massenmedien wird zur qualitativen, da ihre kommunikative Effizienz durch Masse und Realitätsgrad ungleich höher liegt als bei der Bildenden Kunst [...] c) Die Darstellung gesellschaftlichen Bewußtseins vollzieht sich heute außerordentlich effektiv in den optischen Massen-

\footnotetext{
5 Wie Imagewerbung (wahrscheinlich) funktioniert erklärt der Evolutionsbiologe Geoffrey Miller (Miller 2010).

${ }^{6}$ Zur Bedeutung der Bilder in der Werbung vgl. a. Messaris 1997.
} 
kommunikationsmedien; es ist hier von jedermann jederzeit relativ problemlos erfahrbar und mitvollziehbar.“ (Möller 1971: 363f.).

\subsection{Geschichte der Plakatwerbung}

In ihrer umfangreichen Dissertation zur Reklame in Deutschland macht Christiane Lamberty drei Wurzeln der Plakate aus: amtliche Ankündigungen, Buchhändlerplakate und die illustrierten Plakate der Schausteller. ${ }^{8}$ Als um 1850 die ersten Produktwerbeplakate aufkommen, haben sie noch recht eigentümliche Formen: „Produktreklame wurde zunächst nur durch so genannte ,Blankoplakate' gemacht; in fertige Entwürfe wurden nur noch die jeweiligen Firmen- bzw. Markennamen eingefügt. Reisende der Druckereien legten dazu zweimal jährlich den Kunden fertige Plakate zur Auswahl vor." (Lamberty 2000: 189). Gegen Ende des neunzehnten Jahrhunderts wird das Plakat nun als eigenes Kunst- und Werbemedium entdeckt; deutsche Beiträge zur gewerblichen Bildkunst aber lassen zunächst noch auf sich warten. Die ersten innovativen Plakate deutscher Herkunft - Thomas Theodor Heines „Simplicissimus“-Hund und Ludwig Sütterlins Plakat für die Berliner Gewerbeausstellung - wurden im „Durchbruchsjahr“ 1896 veröffentlicht, nachdem die Besucher der Plakatausstellungen in London (Royal Aquarium 1894 und 1896) und Hamburg (Museum für Kunst und Gewerbe 1896) das bereits über zehn Jahre dauernde Schaffen vor allem britischer, amerikanischer und französischer Künstler hatten bewundern können. Nach den Jahren des „Misstrauen[s] gegenüber der Gebrauchsgraphik" eines Grasset oder Mucha als vermeintlich minderwertige Kunstform (Ingenkamp 1996: 167) konnten sich nun München und Berlin als frühe Zentren der jetzt auch über Ausschreibungen angeregten Kunstform herausbilden.

$\mathrm{Zu}$ einer eigenständigen Marke wird das deutsche Plakat aber erst ab 1903, als Hans Lindenstaedt und Lucian Bernhard beginnen, das „Berliner Sachplakat“ zu entwickeln. „Hierbei ging es vor allem um Verkürzung, Komprimierung und Assoziation. Alles als überflüssig empfundene Beiwerk wurde weggelassen; was übrig blieb, suchte man in seinem Informationsgehalt weiter zu komprimieren, so dass vor allem der Textanteil zugunsten des Bildanteils schrumpfte. Assoziative Zusammenhänge zwischen Bild und Text wurden bewusst angestrebt, das Prinzip der ,Wort-Bild-Marke' zum obersten Prinzip erklärt. [...] Die Reduktion und Konzentration hatte nicht nur eine völlig neue Bildwelt zur Folge, sondern auch enorme Auswirkungen." (Grohnert 2007: 78) Zunächst bestand die Auswirkung darin, dass der Jugendstil überwunden wurde. Man kann im Berliner Sachplakat einen Vorläufer der Bauhaus-Werbegrafik sehen, insofern seine Konzeption auf empirische

\footnotetext{
7 Vgl. a. den Beitrag der Adhoc-Gruppe Visuelle Kommunikation im selben Band: Adhoc-Gruppe Visuelle Kommunikation 1971.

8 Als kleine Einführung zu Wesen und Geschichte der Plakatwerbung s.a. Haubl 1992.
} 
Untersuchungen gestützt war und von einer heute nur noch schwer vorstellbaren Publizistik und öffentlichen Diskussion begleitet wurde.

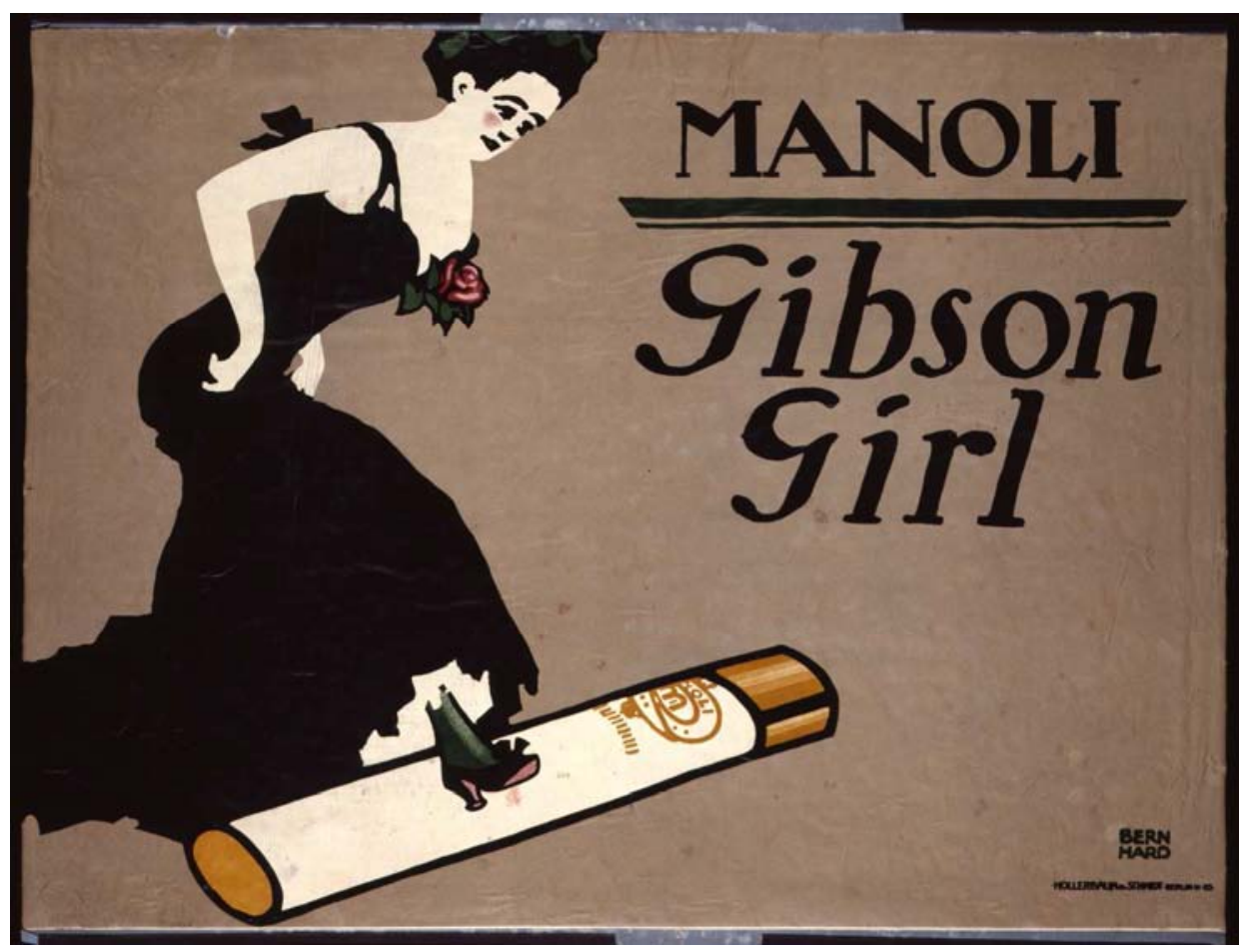

Abb. 1: Kein „Phrasennebel“ (Lamberty 2000: 196): Manoli Gibson Girl (Dame mit Rose). Plakat von Lucian Bernhard, o.J. (vor 1911). Hollerbaum \& Schmidt, Berlin Farblithographie, Steindruck, 69 x 92,5 cm.

Deutsches Historisches Museum Berlin.

1907 rufen je zwölf Künstler und Industrielle zur Gründung des Deutschen Werkbundes auf. „[D]ie Unternehmer zogen aus dem schlechten Abschneiden des deutschen Kunstgewerbes auf den Weltausstellungen und dem verheerenden Urteil, dass dieses im Vergleich zum französischen oder englischen besonders stillos sei und für die Massenproduktion nicht tauge, die Konsequenz, der Einsatz künstlerischer Entwürfe sowohl in der Produktion als auch in der Werbung sei aussichtsreich, um für qualitativ hochwertige Waren Marktchancen zu eröffnen." Im Werkbund wird ein „Programm allgemeiner Geschmacksbildung als das gemeinsame Werk von Produzenten, Künstlern, Einzelhandel und Reklamefachleuten" entworfen.(Lamberty 2000: 329).

In der Symbiose von Kunst und Gewerbe sollte auch die Volkserziehung nicht vergessen werden. 1912 fragt Adolf Saager, der Generalsekretär der Vereinigung Die Brücke: „Wie ist die Reklame zu organisieren, damit das dafür ausgegebene 
Geld den höchsten Nutzeffekt ergibt und zugleich in den Dienst der Kultur gestellt wird?" (Lamberty 2000: 324) Er stellte sich eine Koppelung zwischen Reklamezweck und einem kulturgeschichtlichen, gesundheitserzieherischen oder anders bildenden Text auf der Rückseite aller Reklamebilder vor, „Reklame als Kulturträger, als die mit Riesenmitteln ausgestattete Volkshochschule.“ (ebd.: 325).

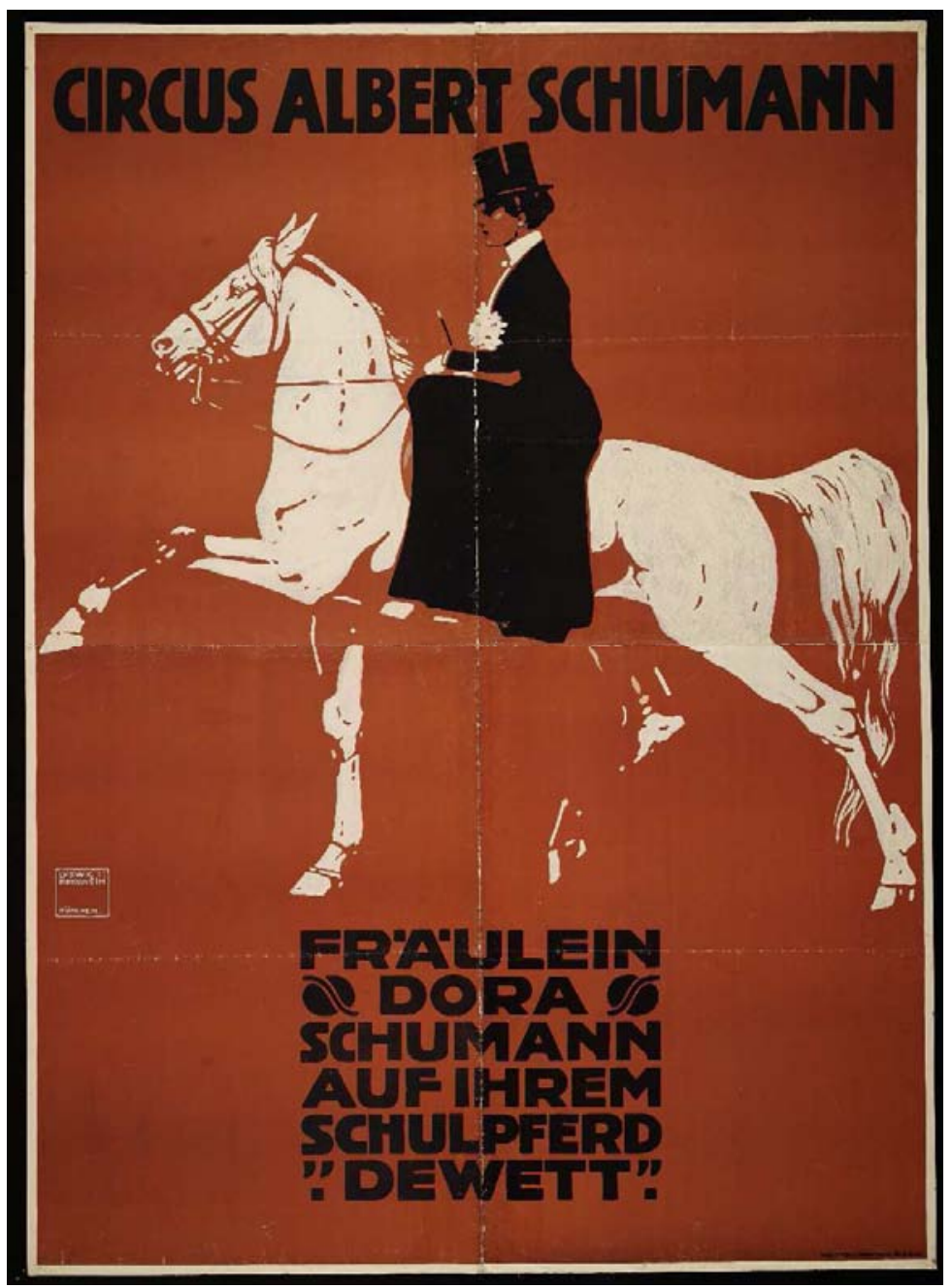

Abb. 2: Mit Ludwig Hohlwein „tritt das Erhabene in die Plakatwelt ein“ (Kühnel 2007: 143), 1910. Circus Albert Schumann - Fräulein Dora Schumann auf ihrem Schulpferd "Dewett", o.J. (um 1910). Vereinigte Druckereien und Kunstanstalten, München. Farblithographie, Steindruck. 242,5 x $176 \mathrm{~cm}$.

Deutsches Historisches Museum, Berlin. 
So lässt sich (auch) anhand der Werbung deutsche Mentalitäts- und Geistesgeschichte nachvollziehen, und Beispiele sind leicht zu finden. Nach der frühen Glanzzeit des Werbeplakats haben viele Künstler ihr Können in den Dienst der politischen Parteien gestellt, weshalb man beim politischen Plakat der Weimarer Republik, wie sich unten zeigen wird, von einer Blütezeit sprechen kann. Zugleich wurde die Wirtschaftswerbung perfektioniert.

1919 kam es zur Gründung des Bundes deutscher Gebrauchsgrafiker, denn „Reklame war vor allem ein Arbeitsfeld dieses Standes geworden, wenngleich nach wie vor die so genannten freien Künstler auf diesem Felde tätig waren [...]. Das hatte durchaus beflügelnde Wirkung. Die verschiedenen Strömungen wie Expressionismus, Dadaismus, Futurismus und Neue Sachlichkeit oder Konstruktivismus hatten Einfluss auf die stilistischen Mittel im Plakat gewonnen." (Kühnel 2007: 143). Im Deutschen Werkbund, im Staatlichen Bauhaus, an der Essener Folkwangschule für Gestaltung wurden gestalterische Positionen, Bildaufbauten und Schriften entwickelt, die zum Teil bis heute von Bedeutung sind.

Für die Zeit von 1933 bis 1945 ist von einer „leichten Faschisierung“ der Werbung gesprochen worden (Ingenkamp 1995: 237-245); festzuhalten ist jedenfalls, dass die Werbewirtschaft im Dritten Reich von der „Gleichschaltung" nicht verschont blieb und klaren nationalsozialistischen Zwecken zu dienen hatte (vgl. Westphal 1989; Rücker 2000). Typisch für die Nachkriegszeit bis Ende der 1950er Jahre waren Plakate mit der Aufschrift „Es gibt wieder...“.

\subsection{Neuere Beispiele}

Gerade im großen und reichen Deutschland scheint es sich nach wie vor zu lohnen, landesspezifische und so nirgends sonst verständliche Werbung zu schalten. So gab es kurz nach dem neunten November 1989 im werbemäßig schon einmal vor-vereinigten Deutschland eine Plakatwerbung von Fiat: Vier offensichtlich ostdeutsche Interessierte schauen sich einen Fiat Panda an. ${ }^{10}$ Darüber steht:

\footnotetext{
„Also mal ehrlich, Erich...äh Egon..., den real existierenden Spätkapitalismus hatten wir uns wesentlich dekadenter vorgestellt. - Fiat Panda. Die tolle Kiste.“ Unter dem Bild heißt es: „Was hatte der Schwarze Kanal nicht alles über den Westen erzählt: kalte Glitzerwelt, verchromte Herzlosigkeit. Und dann fährt man rechts ran, kauft Bananen, und plötzlich fällt es einem wie Glasnost von den Augen: dieser Kleine da, nicht größer als ein Trabi, das soll der Konsumterror sein, vor dem die weise Führung immer gewarnt hat?

${ }^{9}$ Bei der Übernahme der großen Werbefirmen durch amerikanische Konkurrenten sei es zum Verlust jeglicher Eigenständigkeit und damit zur „Amerikanisierung“ der Werbung in Deutschland gekommen, schreibt Konstantin Ingenkamp in seiner Dissertation zum Thema (1996: 266-275), bleibt aber eine genauere Beschreibung des „Amerikanischen“ in der deutschen Werbung schuldig.

${ }^{10}$ Reproduziert auf dem Titelblatt der Geschichtswerkstatt 25 (1992): Werbung als Geschichte.
} 
Lächerliche 4,0 1 bleifrei Super [...] - ist das die spätkapitalistische Vergeudung knapper Ressourcen? 11.990,- DM für 45 PS/33kW, geregelten 3-Wege-Kat., verschiebbaren Aschenbecher und wiederverschließbare Türen - das soll Ausbeutung der werktätigen Massen sein? Der Panda ist lieferbar in Sozialismusrot, Preußischblau, Diplomatenschwarz und weiteren United Colors. [...].“

Die „United Colors“ spielen natürlich auf die italienische Modefirma Benetton und die außerordentlich Aufsehen erregenden, wenn auch letztlich nicht unbedingt einträglichen Plakatkampagnen Oliviero Toscanis an, mit der der Parforceritt durch die Geschichte der Werbeplakate enden soll. Anfang der 1990er Jahre machte eine neue Art Werbung Furore, die gegen die eiserne Regel der Branche zu verstoßen schien, niemals Problematisches darzustellen. ${ }^{11}$ Toscanis Plakate zeigten keine Kleidung (jedenfalls nicht von Benetton), sondern nur Problematisches - einen Säugling voller Schmiere an der Nabelschnur, zwei kopulierende Pferde, eine ölverschmierte Möwe, einen Aids-Patienten im Endstadium, ein Opfer eines Mafiamords, Kondome - und trafen damit einen Nerv. Als Imagewerbung schien seine Kampagne zunächst außerordentlich erfolgreich, indem die vormals „brave“ Benettonkleidung nun zu einer Art globaler Protestkleidung avancierte, deren Träger etwa Toleranz, Entrüstung und Verantwortungsgefühl demonstrierten. Die Öffentlichkeit war gespalten, schließlich blieben die Plakate kommerziell orientierte Werbung. „Unbehagen bereitete es Kritikern, dass es manchen BenettonPlakaten offenbar tatsächlich gelang, eine größere Aufmerksamkeit auf Themen wie Aids oder Rassismus oder auch den Krieg in Bosnien zu lenken, als es andere Medien damals vermochten. Mit der Verwendung von Pressefotos in den Kampagnen von 1992 holte Toscani die Realität in ihren negativen Erscheinungsformen in die heile Welt der Werbung." (Döring 2008: 634).

1994 erschien das wohl berühmteste Plakat: eine blutige Uniform. „Das Foto rief heftigste Kritiken hervor“, Zeitungen lehnten den Abdruck ab. „In Deutschland protestierten Benetton-Händler und meldeten Umsatzeinbußen [...]. Entscheidend für die nachhaltige Wirkung seiner Bilder ist nicht zuletzt, dass Toscani den Betrachter mit seiner Interpretation allein lässt. Er serviert die Klischees frontal und geradezu klinisch rein und fordert damit fast unvermeidlich eine Reaktion heraus.“ (Döring 2008: 635f.). In der zweiten Hälfte der 1990er Jahre verzichtete Benetton auf Imagewerbung, unterstützte aber weiterhin etwa die Formel 1. Zur Trennung von Toscani kam es im Jahre 2000 in der Kontroverse um eine neue Plakatserie, die amerikanische Häftlinge in Todeszellen zeigte. In Insgesamt 120 Ländern waren Benetton-Plakate zu sehen, viele wurden zensiert, manche waren Gegenstand von Gerichtsverfahren, auch in Deutschland.

\footnotetext{
${ }^{11}$ Eine andere, untergeordnete Regel der Werbung ist freilich „Abweichen von der Norm“ (so der Titel des Art-Directors-Club-Jahrbuchs 1998). Hier hat die schwächere die stärkere einmal geschlagen.
} 


\section{Politische Plakate}

„Erste Zeugnisse von Rang verdankte die politische Plakatkunst im kaiserlichen Deutschland der satirischen Zeitschrift ,Simplicissimus“", vor allem dem schon zitierten Thomas Theodor Heine. Käthe Kollwitz' Plakat für die Deutsche Heimarbeit-Ausstellung von 1906 wurde auf Wunsch der Kaiserin von den Anschlagssäulen entfernt, weil ihr das Motiv einer abgearbeiteten Frau nicht gefiel (Malhotra 1984: 17). Kollwitz verstand sich als politische Künstlerin: „In solchen Augenblicken, wenn ich mich mitarbeiten weiß in einer internationalen Gemeinschaft gegen den Krieg, hab ich ein warmes, durchströmendes und befriedigendes Gefühl. Freilich, reine Kunst in dem Sinne wie z.B. die Schmidt-Rottluffs ist meine nicht. Aber Kunst doch. Jeder arbeitet, wie er kann. Ich bin einverstanden damit, dass meine Kunst Zwecke hat. Ich will wirken in dieser Zeit, in der die Menschen so ratlos und hilfsbedürftig sind.“ (zitiert bei Malhotra 1984: 11).

Buchlis Werbe-Definition (s.o) legt eine enge Verwandtschaft von Wirtschaftswerbung und politischer Propaganda nahe. Ästhetisch und personell sind beide Arten eng miteinander verwoben, schon weil ab 1916 (fast) dieselben Grafiker/Künstler die Plakate gestalten. Im dritten Kriegsjahr entschied sich die neue Oberste Heeresleitung unter Ludendorff, nun auch massiv Propaganda zu betreiben und gewann den Werbegrafiker Lucian Bernhard für die Gestaltung des Plakats zur fünften Kriegsanleihe. Bis dahin schien es, „als sei das drastische Feindbild mit einem Tabu belegt. Dieses für uns rätselhafte Verhalten hatte einen Grund: Auf die alliierte Flugblattpropaganda antwortete die Heeresleitung aus moralischen Bedenken nicht.“ (Vorsteher 2007: 124).

\subsection{Politische Plakate der Weimarer Republik}

1918 begann dann die große Zeit des politischen Plakats: „Gleich nach der Novemberrevolution wurde das Land nicht nur mit amtlichen Plakaten überschwemmt, vor allem die politischen Parteien bemächtigten sich nun dieses Mediums. In einer ereignisreichen Zeit des politischen Umbruchs, in der es weder Rundfunk noch Fernsehen gab, die Zeitungen entweder nicht erschienen oder für eine schnelle Information nicht zu gebrauchen waren, sich viele eine solche auch nicht leisten konnten, kam Plakaten eine besonders große Bedeutung zu. Aber nicht nur die turbulenten Ereignisse trugen zu ihrer Vermehrung bei, sondern vor allem die Tatsache, daß die einschränkenden Zensurbestimmungen nun zum größten Teil weggefallen waren. Das Plakat war als schlagkräftiges Agitations- und Propagandamittel in der geistigen Auseinandersetzung und im politischen Kampf, vor allem in den Wahlkampfschlachten, bestens verwendbar.“ (Bayrisches Hauptstaatsarchiv München 1996: 9).12

\footnotetext{
12 Im Bayrischen Hauptstaatsarchiv sind 40.000 Plakate archiviert, davon 25.000 politische, die v.a. auf die „Sammlung Rehse“ zurückgehen.
} 
Die Sprache der Plakate war nach über vier Jahren Weltkrieg, Revolution und alltäglich gewordener blutiger Auseinandersetzung mit politischen Gegnern nicht mehr dieselbe wie zuvor. „[A]bgesehen vom agitatorischen Gestus, mit dem nach 1918 für neu entstandene Parteien und deren Ideen geworben wurde, erschienen oft Schreckensbilder des politischen Gegners, gepaart mit Katastrophenvisionen. Was bis dahin nur in der Karikatur und Pressezeichnung oder im Flugblatt möglich gewesen war, zog nun in die Bildwelt des öffentlichen Anschlags ein: Verunglimpfung, Spott und Abschreckung; ebenso eine Ikonographie, die sich bestimmter Stereotypen für Gut und Böse bediente.“ (Kühnel 2007: 142).

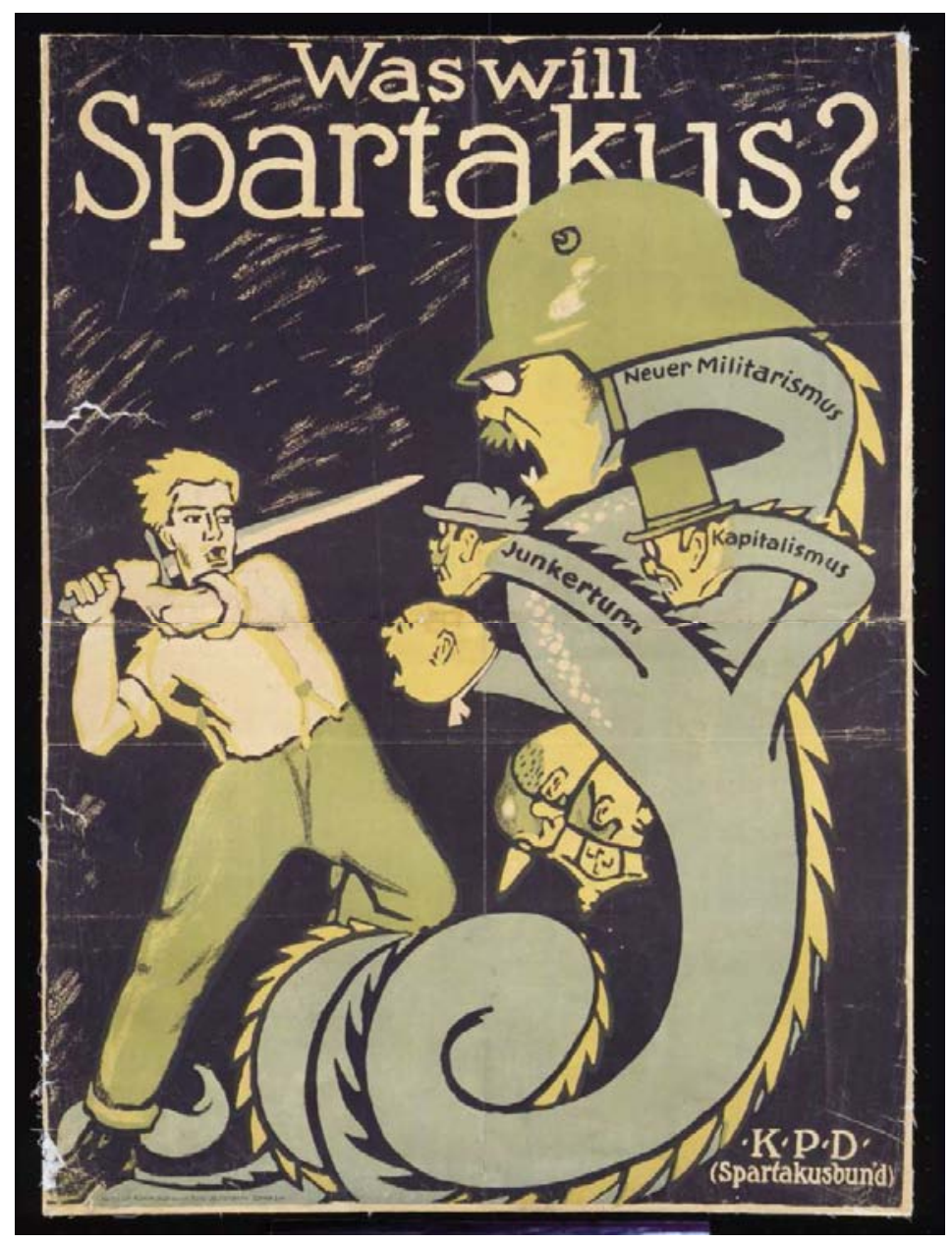

Abb. 3: Was will Spartakus?/K.P.D. (Spartakusbund), 1919.

Deutsches Historisches Museum Berlin. 
Manfred Hagen (21984: 49) schreibt zur Bedeutung der Plakate, sie seien „Rufe auf Papier“ gewesen. „Was heute in der westlichen Welt meist kurz vor Wahlterminen als Plakate von Parteien sichtbar wird, das sind nur blasse Nachkommen einer fast ein Jahrhundert lang blühenden, äußerst mannigfaltigen und oft aussagestarken Propagandaform." Wie schon das Berliner Sachplakat ist auch das politische Plakat auf die Eigenheiten des Passanten der Großstadt zugeschnitten. „Sein Adressat ist nicht der lesende, zuhörende, in Ruhe nachdenkende Mensch, der sich vorsätzlich politischen Fragen widmet, sondern der unterwegs Befindliche, der zur Arbeit, zum Einkauf oder zum Termin eilt. Dieses Vorübergehen nutzt das Plakat. Es soll den Menschen, der sich mit dem intendierten Appell möglicherweise sonst nicht befassen würde, mit optimal platzierten und großformatigen Aufrufen gleichsam im Flug ansprechen, die nur einige Schritte währende Aufmerksamkeitsspanne maximal nutzen und schnellstens eine ,Botschaft' vermitteln. [...] Die Notwendigkeit, einen möglichst komprimierten Appell auszusenden, hat in diesem Sinn, massenpsychologische Meisterwerke hervorgebracht..." (ebd.). Vielleicht ist das auch der Grund für die Armut so vieler heutiger (Groß-)Plakate; so sie nicht etwa an Bahnhaltestellen angebracht sind, richten sie sich an Autofahrer, die nur noch Bruchteile von Sekunden zur Betrachtung haben. Viel lässt sich in dieser Zeit nicht vermitteln.

Alle während der Weimarer Zeit bedeutenden Parteien (von links nach rechts: KPD, SPD, DDP, Zentrum, DVP, BVP, DNVP, NSDAP) nutzten auf ihren Plakaten drastische Bilder und Sprüche. Ein Historiker hat 1939 in seiner Dissertation über Wesen und Bedeutung der Plakate in der „Kampfzeit“ die „Gesetze der publizistischen Massen- und Volksführung, zitiert und zusammengetragen nach Ernst Dovifat und Adolf Hitler" zusammengetragen: 1. Humanität und Schönheit können wegen des „herrschenden Existenzkampfes der Völker [...] nicht als Maßstab für Propaganda Verwendung finden“; 2. Propaganda „hat sich ewig nur an die Masse zu richten“. Daraus folgen die Grundgesetze der geistigen Vereinfachung, der Stoffbeschränkung, der „hämmernden Wiederholung“, der Subjektivität, der gefühlsmäßigen Steigerung etc. (Medenbach 1941: 12-14). Die Bedeutung des Plakats ist für ihn auch nach sechs Jahren Diktatur noch gegeben: Es sei „eine der der zahlreichen Waffen, die heute der moderne Publizist zur geistigen Formung und Führung der Massen und Völker einsetzt." Und anders als Film, Zeitung oder Rundfunk sei es „zu jeder Zeit wirkungsbereit. Es kann jeden erfassen, der auf die Straße hinausgeht, ob zur Arbeit oder zum Spaziergang nach Feierabend.“ (ebd: 1,3$) \cdot{ }^{13}$

\subsection{Politische Plakate der Bundesrepublik}

Nach dem zweiten Weltkrieg nehmen die politischen Plakate eine andere Gestalt an; das Umdenken der „Stunde Null“ war allerdings nicht ganz so radikal, wie man

${ }^{13} \mathrm{Zu}$ politischen Plakaten vgl.a. Brommer; Krümmel; Werner 2002. 
heute meinen mag: „Zwar hatte der Holocaust den biologischen Rassismus weitgehend diskreditiert; ein kultureller Rassismus indes schimmerte weiterhin durch, wenn Sowjetbürger als Menschen niederer Kultur, als verdreckt, gewalttätig und eroberungssüchtig präsentiert wurden, und auch Reminiszenzen an den biologischen Rassismus erhielten sich in einzelnen Darstellungen [...].“ (Paul 2008: 95). In Erinnerung geblieben ist vor allem das CDU-Plakat von 1953.

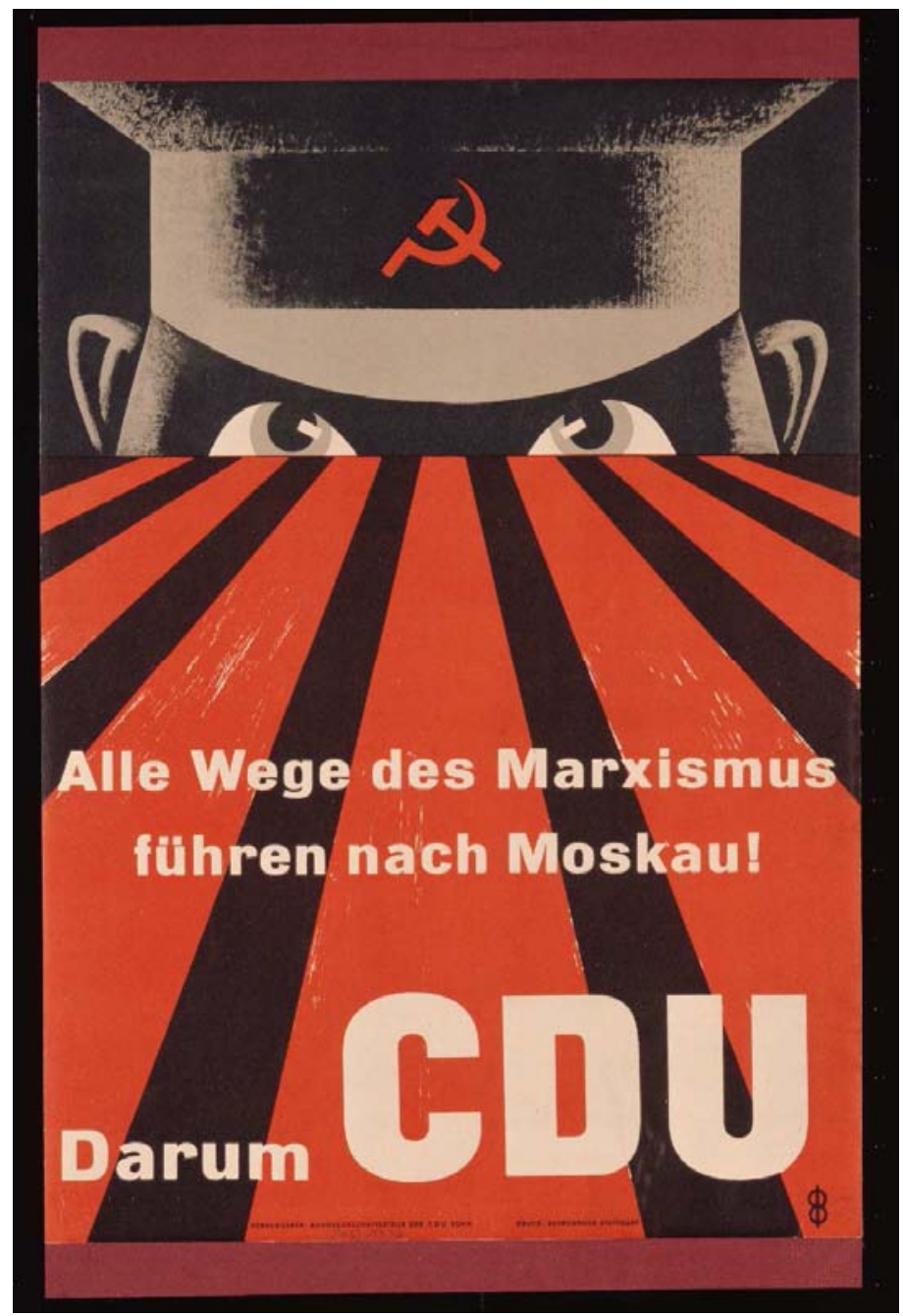

Abb. 4: Ein „Klassiker“ des politischen Plakats: CDU-Werbung von 1953. Deutsches Historisches Museum Berlin. 
„Wie kein anderes Bild transferiert das Plakat das bis dato figürlich angelegte antikommunistische Feindbild in eine moderne zeitgenössische Bildsprache, indem es dieses mit dem Bild einer strukturell-totalitären Bedrohung verkoppelt. Ähnlich wie bei dem Foto vom Torhaus Auschwitz-Birkenau erzeugt das Plakat einen energetischen Kraftraum, der sich sowohl aus der zentralperspektivischen Anordnung seiner Bildelemente wie aus dem hypnotischen Blick des bolschewistischen Funktionärs ergibt, der die mit dem Sowjetsystem assoziierten Überwachungs- und Bespitzelungsängste aktiviert.“ (Paul 2008: 90)

Die politischen Plakate wurden schon bald nach Gründung der Bundesrepublik nicht mehr aus dem Bauch heraus konzipiert: „Seit Beginn von Adenauers Kanzlerschaft erforschte das Institut für Demoskopie in Allensbach im Auftrag der Regierung fortlaufend die öffentliche Meinung über Adenauer; vor Wahlen wurden diese Untersuchungen durch EMNID-Studien auf Kosten der CDU ergänzt. Allensbach-Chef Peter Neumann gehörte auch bei der Bundestagswahl von $1957 \mathrm{zu}$ den wichtigsten Beratern, denn es galt, die Kanzlerdarstellung an die Umfragen anzupassen. Im Herbst 1956 sprachen sich nur 34 Prozent der Befragten für eine erneute Wahl Adenauers aus, was gegen eine starke Personalisierung des Wahlkampfes sprach. Nachdem jedoch seine Beliebtheit nach der Niederschlagung des ungarischen Volksaufstands durch sowjetische Truppen in den internen Meinungsumfragen wieder steil anstieg, setzte die Wahlkampfleitung erneut ganz auf den Kanzler. Deutlich negativ schlug in den Umfragen lediglich zu Buche, dass Adenauer als zu alt angesehen wurde.“ (Bösch 2008: 196). Also wurde er für das berühmte Plakat verjüngt.

Hubert Straufs Essener Agentur „Die Werbe“, die das Plakat gestaltet hat, zeichnete auch für den Coca-Cola-Spruch „Mach mal Pause“ verantwortlich. „In ihrer sprichwörtlichen Eingängigkeit wurden [die beiden Sprüche] zu den mentalen Imperativen der spätern Adenauerzeit.“ (Gries; Ilgen; Schindelbeck 1995b: 94). „Das ,Keine-Experimente-Plakat zeichnet sich zudem durch eine Konzentration auf das Wesentliche aus. Der Verzicht auf einen Hintergrund und die alleinige Fokussierung auf Adenauers Kopf waren ebenso auffällig wie die prägnante Kürze des Slogans. Dies korrespondierte mit Adenauers politischer Gabe, komplexe Sachverhalte zu vereinfachen, zuzuspitzen oder verschmitzt schön zu färben. Das Wahlplakat von 1957 war in gewisser Weise ein Ausdruck seiner pointierten Rhetorik, die etwa im gleichen Jahr die Aufstellung von Atomwaffen als weiterentwickelte Artillerie rechtfertigte.“ (Bösch 2008: 197). In seiner Einfachheit ist das Plakat raffiniert gestaltet. „Die semantische Ähnlichkeit mit der Wahlkampfaussage der Union aus dem Jahr 1986/87, Weiter so, Deutschland!' ist frappierend. ,Weiter so Deutschland' aber entwickelte längst nicht jene dramaturgische Spannung, die die Text-Bild-Komposition ,Keine Experimente ${ }^{6}$ - auch aufgrund ihres historischen Ortes - auszeichnete: Den aufrüttelnden Worten auf der einen Seite antwortete das beruhigende Bildnis des Alten auf der anderen, ein in sich geschlossenes Zeichensystem, das zugleich Ansprache und Integration leistete. Noch waren ja die Erinnerungen an Zusammenbruch und unmittelbare Nachkriegszeit in der 
Bevölkerung wach, selbst bei den damals jüngsten Wählern, den Einundzwanzigjährigen. Verglichen mit jener Zeit mußten die Zustände nun, im Jahr 1957, als geradezu paradiesisch empfunden werden. Nur vor dem mentalen Hintergrund dieses Kontrastes ist es zu verstehen, warum eine Ex-negativo-Formulierung als die positivste aller Möglichkeiten verstanden werden mußte. Der Gestus, mit dem „Keine Experimente' auftrat, scheint zudem in sich etwas von der Würde, Qualität und Festigkeit eines kategorischen Imperativs zu enthalten." (Gries; Ilgen; Schindelbeck 1995b: 103).

Die Gestaltung von Wahlplakaten erscheint heute weitgehend uniformiert. Meist wird mit dem Konterfei eines Politikers geworben, der Schriftanteil ist auf wenige Wörter beschränkt. Das ist auch bei den Grünen nicht anders; ihr zunächst „alternativer", farbenfroher Bildcode hat sich mit den Jahren zu einem eher postmodernen entwickelt. „Während die alternative ,Öko-Ästhetik ${ }^{`}$ der Ursprungswähler sich dadurch auszeichnete, dass man der ,kalten Modernisierung' mit organischer Buntheit und mit authentischer Individualität begegnete, war der postmoderne Code der ,No-future'-Generation in den 1980er Jahren von pessimistischer Abgeklärtheit gekennzeichnet.“ (Fahlenbrach 2008: 480). Nur die Plakate, die zur Direktwahl des Berliner Bundestagskandidaten Christian Ströbele aufrufen, haben sich einen eigenen Stil bewahrt. „Die Kreuzberger Wähler werden hier als Mitglieder einer fröhlich rebellierenden, aber auch pluralistischen Alternativkultur angesprochen, in der Jung und Alt, Christen und Moslems, schwarzhäutige und weißhäutige Menschen friedfertig für gemeinsame Werte eintreten. An ihrer Spitze steht Ströbele, dessen sozialpolitisches Programm für mehr Gerechtigkeit auf einem Wahlplakat im Plakat verkündet wird: ,Sozial gerecht ist nicht zu teuer mit Ströbeles Vermögenssteuer!' und: ,Prenzel-, Kreuzberg, Friedrichshain wählen sich den Christian rein'. Auch der gereimte Duktus dieser Slogans zitiert die Agit-PropÄsthetik der linken Protestkultur. Sowohl in der Ästhetik als auch mit den proklamierten Werten und Zielen spricht Seyfrieds Plakat also u.a. jene Stammwähler an, die sich nicht nur mit der Politik, sondern auch mit der alternativen Kultur identifizieren, für die die Grünen politisch und symbolisch seit den 1980er Jahren stehen - wenn auch mit Anpassungen an postmoderne und andere Werteentwicklungen“" (ebd.: 481). Jedes politische Plakat spricht auch von seiner Entstehungszeit: das SPD-Plakat von 1949 - „Mit der SPD von Bonn über Berlin für ein freies, soziales und geeintes Deutschland“, und zwar in den Grenzen von 1937; das SED-Plakat von 1950 mit Marx, Engels, Lenin und (noch!) Stalin im Hintergrund; oder, und das leitet zum „kritischen“ Teil über, das Antiplakat der Piratenpartei mit dem Slogan: „Vertrau keinem Plakat. Informier dich.“

\section{Analyse, Kritik und Protest}

Die Kritik der Werbung in Plakat- oder anderer Form hat eine lange Tradition. Bereits vor dem ersten Weltkrieg wurde sie vereinzelt als „Zeichen des amerika- 
nischen Kapitalismus“ oder gar „jüdischer Erwerbsgier“ verschrien, und Preußen hat 1902 und 1907 Gesetze gegen die „Verunstaltung von Ortschaften und landschaftlich hervorragender Gegenden" verabschiedet (Lamberty 2000: 477-490). Erst in jüngerer Zeit aber wird Werbung als Transportmittel einer Ideologie verstanden. Im ersten Band seines großen, zwischen 1938 und 1947 entstandenen Werkes „Das Prinzip Hoffnung" versteht Ernst Bloch die Werbung in Schaufenstern und auf Plakaten noch recht unkritisch als „Leimruten für die angelockten Traumvögel.“ „Die Reklame macht aus der Ware, auch aus der beiläufigsten, einen Zauber, worin alles und jedes gelöst ist, wenn man sie nur kauft. Die Dame der Zeichnung, die Kölnisch Wasser auf die Schläfen tupft, die von Herren eine Schweizer Schokolade entgegennimmt, ist eben dadurch die Glücklichgewordene schlechthin." (Bloch 1985: 400). Etwa zur gleichen Zeit notierte Theodor W. Adorno in den „Minima Moralia“:

„Welch einen Zustand muß das herrschende Bewußtsein erreicht haben, daß die dezidierte Proklamation von Verschwendungssucht und Champagnerfröhlichkeit, wie sie früher den Attachés in ungarischen Operetten vorbehalten war, mit tierischem Ernst zur Maxime richtigen Lebens erhoben wird. Das verordnete Glück sieht denn auch danach aus [...]. Die Ermahnung zur happiness, in der der wissenschaftlich lebemännische Sanatoriumsdirektor mit den nervösen Propagandachefs der Vergnügungsindustrie übereinstimmt, trägt die Züge des wütenden Vaters, der die Kinder anbrüllt, weil sie nicht jubelnd die Treppe hinunterstürzen, wenn er mißlaunisch aus dem Geschäft nach Hause kommt. Es gehört zum Mechanismus der Herrschaft, die Erkenntnis des Leidens, das sie produziert, zu verbieten, und ein gerader Weg führt vom Evangelium der Lebensfreude zur Errichtung von Menschenschlachthäusern so weit hinten in Polen, daß jeder der eigenen Volksgenossen sich einreden kann, er höre die Schmerzensschreie nicht. Das ist das Schema der ungestörten Genußfähigkeit. Triumphierend darf die Psychoanalyse dem, der es beim Namen nennt, bestätigen, er habe halt einen Ödipuskomplex."

Er empfiehlt eine kathartische Methode, um „die Menschen zum Bewußtsein des Unglücks, des allgemeinen und des davon unablösbaren eigenen, zu bringen [...]“ (Adorno 2003: 69f.). ${ }^{14}$

Sein Kollege Herbert Marcuse ging zwei Jahrzehnte später, als Adornos Schriften von einer neuen medienkritischen Generation wiederentdeckt wurden, so weit zu sagen, „die bloße Abwesenheit aller Reklame und aller schulenden Informationsund Unterhaltungsmedien würde das Individuum in eine traumatische Leere stürzen, in der es die Chance hätte, sich zu wundern, nachzudenken, sich (oder vielmehr seine Negativität) und seine Gesellschaft zu erkennen. Seiner falschen Väter,

${ }^{14}$ Der zitierte Teil der Minima Moralia entstand 1944, das gesamte Werk wurde 1951 veröffentlicht. 
Führer, Freunde und Vertreter beraubt, hätte es wieder sein ABC zu lernen. Aber die Wörter und Sätze, die es bilden würde, könnten völlig anders ausfallen, ebenso seine Wünsche und Ängste.“ [...] Mehr noch: „Das Nicht-Funktionieren des Fernsehens und verwandter Medien“ könnte „den Zerfall des Systems“ erreichen. (Marcuse 1967: 256f.) ${ }^{15}$

Ein politisch weniger radikaler, dafür umso auflagenstärkerer Klassiker der Werbekritik stammt aus den 1950er Jahren. Vance Packard (1962)16 dokumentiert in seinem Bestseller über die „Geheimen Verführer“ die Anfänge der angewandten Werbepsychologie mit Befragungen und Motivanalysen, wie sie zur gleichen Zeit auch in der Politik begannen, Anwendung zu finden. Seine Beispiele betreffen etwa die (vermeintlich) sexuellen Konnotationen des Rauchens und des Milchkonsums, oder er deckt die heute weitgehend bekannten (wenn auch immer wieder verdrängten) Kaufmotivationen auf: Statt Autos, Schuhen, Apfelmus usw. kaufen wir Liebes- und Prestigeobjekte bzw. Gefühle und Sehnsüchte wie Verwurzelung, Unsterblichkeit, Anerkennung und andere. Auch dass Werbung den Bedarf erst schafft, den die immer neuen Produkte dann decken, dass sie bewusst auf Kinder abzielt, Impulskäufe erregt usw. ist heute einerseits so normal, dass es niemand mehr sieht oder für erwähnenswert hält, andererseits ist auch das Publikum klüger geworden; der Abstumpfung gegen die Werbung wird also mit mehr Werbung begegnet. ${ }^{17}$

Neben der amerikanischen und der „Frankfurter“ Richtung gibt es eine facettenreiche französische Tradition der Werbekritik. Spätestens seit Roland Barthes' „Mythen des Alltags“ werden die Werbung und die Dinge, für die sie wirbt, als gewichtige und kunstvolle Bedeutungsträger verstanden (Barthes 1957). ${ }^{18}$ In den (auch) konsum- und medienkritischen 1960er Jahren haben die Situationisten, besonders Guy Debord, den „Spektakel“-Charakter der Werbung und ihre Bedeutung für das weitgehend reibungslose Funktionieren der kapitalistischen Gesellschaften deutlich gemacht (Debord 1967, Baudrillard 1970, Debord 1988).

Seit den 1980er Jahren hat unter anderem François Brune in zahlreichen Analysen sprachliche und inhaltliche Einflüsse der omnipräsenten Werbung im medialen Alltag aufgedeckt, nicht zuletzt in der Politik (Brune 1985; 1996, 2004; Offensive 2010: 85-93). Für ihn ist Werbung das neue Gesicht des Totalitarismus, indem sie nach völliger Durchdringung jedes Bürgers strebt, ihm Werte und Vorstellungen von Normalität vermittelt, zwischen Besitzern/Angehörigen und

\footnotetext{
15 Das Original erschien 1964.

16 Das Original erschien 1957.

17 Schon Aldous Huxley (1958) kritisiert, Packards Entdeckungen (die sexuellen Konnotationen des Rauchens und andere (tiefen)psychologische Erkenntnisse, die die Werbeindustrie zur Förderung des Verkaufs anwendet) seien doch lange bekannt. Zu möglichen „Instinkten“ hinter unseren Kaufentscheidungen vgl. Miller 2010.

18 Die deutsche Ausgabe ist 1964 erschienen. Vgl. auch die anlässlich des 50. Jubiläums erschienene Sammlung neuer Mythen (Garcin 2007).
} 
Besitzlosen bzw. Dissidenten teilt, um zu herrschen. ${ }^{19}$ Andere Werbegegner werfen ihr neben Umweltzerstörung und allgemeiner Verschandelung der Landschaft auch die Unterminierung der Demokratie, die Unterwerfung der Presse und nicht zuletzt die Zerstörung kultureller Traditionen vor. ${ }^{20}$ Und die Entscheider/innen in den Machtpositionen sind sich der Bedeutung und Wirkmechanismen der Werbung durchaus nicht ganz unbewusst. Patrick Lelay, ehemals Chef des privatisierten französischen Fernsehsenders TF1, hat es für sein Medium in einem unbedachten Moment auf die griffige Formel gebracht, der Auftrag des Fernsehens sei es, die Gehirne für Coca-Cola empfänglich zu machen. ${ }^{21}$ Trotzdem ist Werbekritik medial nicht präsent, sind die Medien, auch die öffentlich-rechtlichen, doch zu einem wesentlichen Teil durch Werbung finanziert.

Man muss sich die Ausmaße des Phänomens Werbung einmal verdeutlichen: die Einwohner industrialisierter Gesellschaften empfangen heute täglich mindestens 1.500 größtenteils unbewusst wahrgenommene Werbebotschaften, etwa 350.000 in der Kindheit, und Millionen über das Leben verteilt (Ramonet 2000: 38). Ein Informations- und Aktionshandbuch aus dem besonders betroffenen Nachbarland Frankreich setzt die Tagesdosis sogar doppelt so hoch an und fragt: „Ist es möglich, dass das völlig harmlos für uns ist?" (Les Désobéissants 2009: 8). In Frankreich gibt es mehr als eine Million Werbetafeln, davon ist ein Drittel illegal. Jedes Jahr landen 35 Kilo Werbeprospekte im Briefkasten. Die Kosten betragen dreißig Milliarden Euro, das bedeutet 500 Euro pro Einwohner, die Kinder eingerechnet. In einer Umfrage aus dem Jahre 2007 sprachen sich 30\% der Franzosen gegen die Werbung aus, die Hälfte fand sie gefährlich, und 58\% aggressiv. Trotzdem gibt es mit Forcalquier nur ein einziges werbefreies Dorf im Nachbarland (Les Désobéissants 2009: 28) - leuchtendes Beispiel für die Werbegegner ist die seit 1.1.2007 werbefreie 11-Millionen-Metropole Sao Paulo.

Werbekritik muss nicht Totalablehnung sein, sondern kann sich auch als eine Art feuilletonistische Kulturkritik verstehen. Ein erster Schritt ist in jedem Fall die genaue Lektüre. Eine berühmtes, auch als Zeitungsanzeige erschienenes Plakat mit dem früheren Staatschef Michail Gorbatschow etwa zeigt dem aufmerksamen Betrachter einige Ungereimtheiten: Der jetztzeitliche „Gorbi“ sitzt in einem ZIL-

\footnotetext{
${ }^{19}$ Ulrich Eicke hat in diesem Zusammenhang schon vor zwanzig Jahren von einem „Angriff auf unser Bewusstsein“ und dem „Ausverkauf der Politik“ gesprochen (Eicke 1991, v.a. 208-226). Seither ist der Ton der Kritik beißender geworden.

${ }^{20} \mathrm{Zu}$ aktueller ökologischer, sozialer, psychologischer, ethischer Werbekritik, -karikatur und -parodie vgl. das kanadische englischsprachige Magazin „Adbusters“, das Jahresheft der französischęn Organisation „Casseurs de pub“ sowie die an sie angeschlossene Monatszeitung „La décroissancẹ le journal de la joie de vivre“, die auch aktuelle Entwicklungen beobachtet und einschlägige Literatur rezensiert, sowie im WWW die Seiten casseursdepub.org, ladecroissace.net, deboulonneurs.org, paysagesdefrance.org, antipub.org.

${ }^{21}$ Das Zitat im Wortlaut: „Le métier de TF1, c'est d'aider Coca-Cola, par exemple, à vendre son produit (...). Pour qu'un message publicitaire soit perçu, il faut que le cerveau du téléspectateur soit disponible. Nos émissions ont pour vocation de le rendre disponible: c'est-à-dire de le divertir, de le détendre pour le préparer entre deux messages. Ce que nous vendons à Coca-Cola, c'est du temps de cerveau humain disponible.“ Zitiert nach: Les Désobéissants 2009: 4.
} 
41047, einer russischen Staatskarosse aus der Zeit der Perestroika, ist allein, wirkt deprimiert. Er fährt die Mauer ab, oder was von ihr noch steht. Sehnt er sich in die 1980er Jahre zurück? Aus seiner Louis-Vuitton-Tasche, für die das Plakat wirbt, schaut ein Buch über den ehemaligen KGB-Agenten Litvinenko, der 2006 nach einigen unbequemen Recherchen an einer Pollonium-Vergiftung starb. Gorbatschow hat zu diesem Detail geäußert, er habe nicht gewusst, was in der Tasche stecke. (Oeil-de-Boeuf 2010: 62-67). ${ }^{22}$ Beispiele von sexistischer, infantilisierender oder einfach nur ,schlecht gemachter“ Werbung lassen sich angesichts der Omnipräsenz der Plakatwände leicht finden, auch ist es lohnend, sich mit der Arbeit des deutschen Werberats und anderer selbstregulierender Institutionen zu beschäftigen. $^{23}$

\section{Protestplakate}

Der bekannteste deutsche Protestplakatkünstler ist ohne Zweifel Klaus Staeck, der sich seit nunmehr vierzig Jahren wie kein anderer mit der Kunstform befasst. ${ }^{24}$ Protestplakate stellen gewissermaßen die Synthese zwischen politischem bzw. Wirtschaftswerbeplakat und der Kritik an ihr dar; der Übergang zum engagierten Kunstplakat ist dabei fließend: „Die Frage, ob die Plakate denn nun Kunst oder Politik seien, wird bis heute gestellt. Dieses für Menschen aus dem politischen Raum eher belanglose Problem beschäftigt vor allem den Kunstbetrieb und sein weitgehend an traditionellen Kunstauffassungen orientiertes Publikum, das den Kunstraum gern von jeder politischen Einflussnahme freihalten möchte. Dabei sind meine Arbeiten stets sowohl Kunst als auch Politik: eine ständige Gratwanderung, ein Versuch politisch-gesellschaftlicher Meinungsäußerung mit künstlerischen Mitteln, ohne der Diktatur des Ästhetischen unterworfen zu werden.“ (Staeck 2008: 111). Energisch vertritt er die Rolle der Kunst. „Dabei geht es nicht um eine Kunst im Sinne politischer Ergebenheitsbekundungen oder einklagbarer Sozialhilfe, eher um Herausforderung, Reflexion, um ein Korrektiv, immer aber um Teilhabe, nicht um eigennützige Verweigerung. [...] Kunst als Vorausschau, als Warnung, gar als Vision, als Utopie. Was ist aus diesem Anspruch geworden, woher kommt der Hochmut, mit dem manch einer heute verächtlich auf die Politik herabschaut? Gegenwärtig hat die herrschende Politik von Seiten der Kunst nichts Ernsthaftes zu befürchten [...].“" (ebd.: 133).

\footnotetext{
22 Die französische Wochenzeitung „Charlie Hebdo“ hat eine werbekritische Rubrik; auch die eingangs erwähnten Plakatsatiren der deutschen Zeitschrift „Titanic“ lassen sich als Werbekritiken lesen.

${ }^{23}$ Vgl. für Deutschland: www.werberat.de; für Großbritannien: www.asa.org.uk (Seite der Advertising Standards Authority); für Frankreich: www.arpp-pub.de (Seite der autorité de régulation professionelle de la publicité).

${ }^{24}$ Ernst Volland hat eine Zeitlang ähnliche Plakate geschaffen, sich dann aber anderen Medien wie Karikatur, Comic, Film und historischer Fotografie zugewandt.
} 


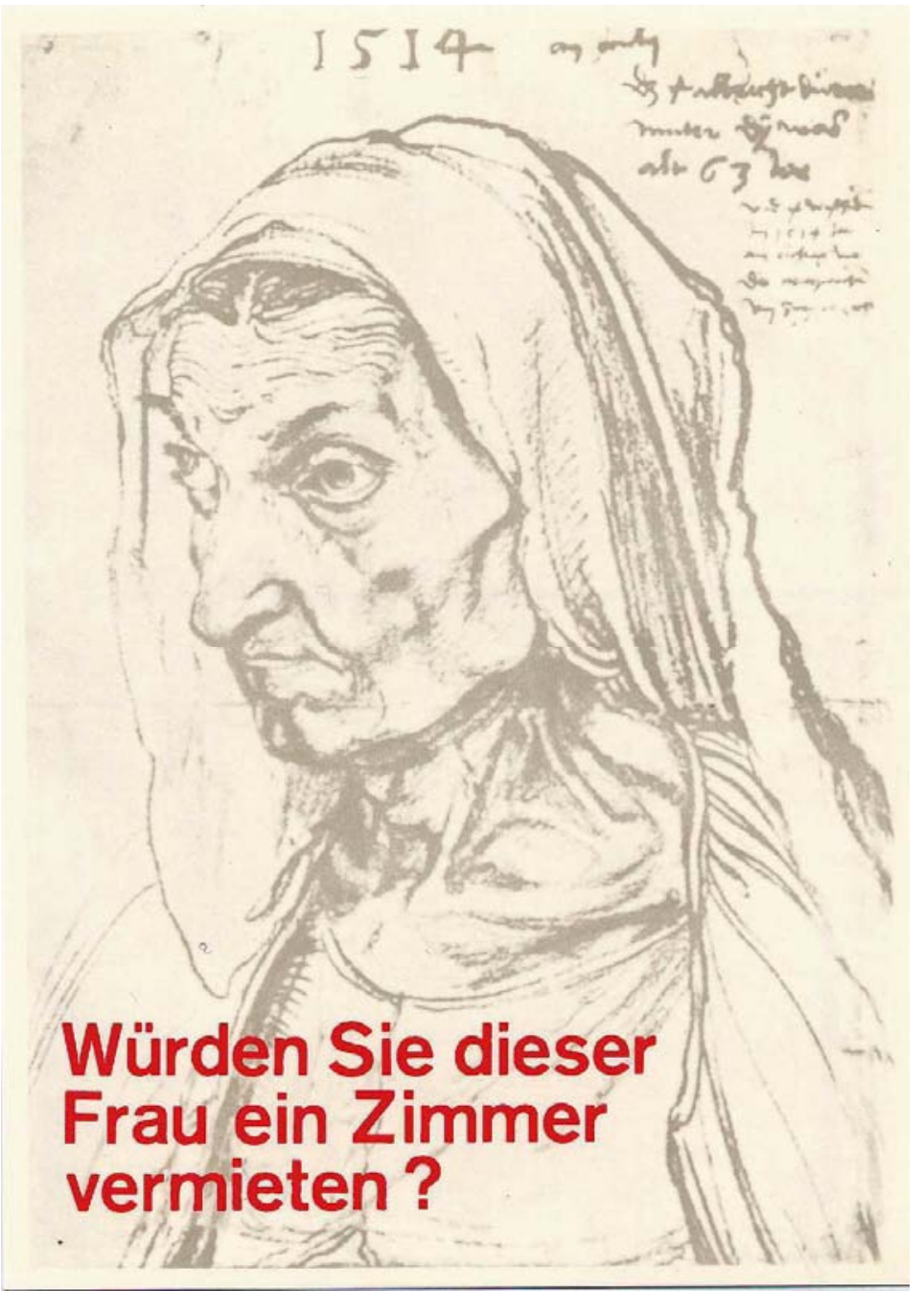

Abb. 5: Das erste Staeck-Plakat (1972).

Weil öffentliche Plakate nicht von jener „künstlichen Aura“ der musealen oder in Galerien ausgestellten Kunst umgeben seien, beschäftigten sich die Betrachter sehr viel stärker mit dem Inhalt. So will Staeck „Denkanstöße geben, Unbequemes zur Sprache bringen, Vorurteile erschüttern, die Kritikfähigkeit möglichst vieler Menschen schärfen. [...] Meine Arbeiten dienen der Aufklärung. [...] Es geht um ein neues kritisches Sehen. Mit Bildern kann man dazu beitragen. Es wäre sinnlos, alle psychologisch ausgeklügelten Bilderlügen etwa in der Werbung einzeln widerlegen zu wollen. Es genügt, einmal gelernt zu haben, den Bildern zu mistrauen, sie mit anderen Augen zu sehen, hinter die Bilder zu blicken." (ebd.: 117; 118f). 
Staecks Vorbilder sind John Heartfield und George Grosz, deren Montagetechnik er weiterentwickelt. Seine scharfe Satire wird nicht von jedem verstanden. „So wurde 1972 das Plakat ,Die Reichen müssen noch reicher werden. Deshalb $\mathrm{CDU}^{\prime}$ gleich mehrfach juristisch verfolgt, weil viele Mitglieder und Anhänger der Union glaubten, das Motiv stamme tatsächlich von der Partei. Die CDU fühlte sich verpflichtet, ihr Klientel zu schützen und es vor einem folgenschweren Irrtum zu bewahren. Als besonders erfolgreich hat sich die Verwendung fiktiver Bekanntmachungen, Amtsbezeichnungen, Wappen und Siegel erwiesen." (ebd: 113). Insgesamt wurden einundvierzig Prozesse gegen ihn geführt, doch er hat alle gewonnen: „Die Hoffnung auf ein Verbot scheint allemal größer als das Vertrauen auf die Stärke der eigenen Argumente.“ (ebd.: 121) Über eine eigene Druckerei, die er mit seinem Bruder und einem Freund betreibt, bewahrt er sich die größtmögliche Unabhängigkeit. ${ }^{25}$

Im Zusammenhang von Werbekritik und Protestplakaten bleiben noch die Aktionen nicht-kommerzieller Nichtregierungsorganisationen zu erwähnen - die doppelte Verneinung zeigt schon an, dass sie eher die Ausnahme als die Regel sind. Vor allem die Tierschutzorganisation PETA sorgt regelmäßig mit ihren Plakataktionen für Aufsehen. In Deutschland treten u.a. Thomas D., Bill und Tom Kaulitz oder Bela B. und Franka Potente für Vegetarismus und gegen das Tragen von Pelzen ein. ${ }^{26}$ Anders (vielleicht) als etwa bei WWF oder Unicef, deren Werbekooperationen u.a. mit Erdölkonzernen als „Greenwashing“ kritisiert werden, greifen die Plakate dieser Tierschutzorganisation sehr viel tiefer. Hier geht es nicht um Kaufentscheidungen zwischen A und B, auch nicht um Spendenmittelwerbung, sondern um einen tief greifenden Bewusstseinswandel; als Verzichtaufruf und Konsumkritik verstanden sind die Plakate sogar potentiell systemgefährdend. ${ }^{27}$

\section{Anwendungen}

Folgendes sollte klar geworden sein:

1. Plakate sind ein eigenständiges visuelles Medium der Beeinflussung.

2. So ist es nach der Werbe-Formulierung Hanns Buchlis (1962: 64) ihr Ziel, die Menschen zu veranlassen, etwas freiwillig zu tun - zweckfreie Kunst- oder Dekorationsplakate sind im öffentlichen Raum ausgesprochen selten, und Informationen werden gemalt („Plakate ankleben verboten“) oder in Form von Schildern vermittelt.

\footnotetext{
$25 \mathrm{Vgl}$. auch www.staeck.de und die heute nur noch antiquarisch zu erstehenden so genannten „Staeckbriefe“.

${ }^{26} \mathrm{Vgl}$. www.peta.de, www.peta.org.

27 PETA ist die bekannteste unter den wenigen Nichtregierungsorganisationen, die überhaupt Plakatwerbung betreiben; die meisten anderen, nicht wenige von ihnen kirchlich, werben vor Weihnachten um Spenden.
} 
3. Kunst-, ereignis- und v.a. mentalitäts- und alltagsgeschichtlich sind Plakate historische Quellen allererster Güte. Überall, wo deutsche Geschichte seit etwa der Thronbesteigung Wilhelms II. vermittelt wird, können und sollten Plakate eine Rolle spielen, also auch im Landeskundeunterricht.

4. Das politische ist aus dem Werbeplakat hervorgegangen; beide stützen sich weitgehend auf die gleichen Ausdrucksweisen und „Macher“. Eine wichtige Ausnahme stellen die Protestplakate Klaus Staecks und einiger Nichtregierungsorganisationen dar, die Großkonzerne, Korruption und/oder die Konsumgewohnheiten der Gesellschaft anprangern und ein Umdenken erzwingen wollen.

5. Kritiker bezeichnen (Plakat)Werbung als hässlich, sexistisch, umweltfeindlich, systemerhaltend, totalitär u.v.m, mit unterschiedlichen Schwerpunkten je nach intellektueller Herkunft. Es bleibt festzuhalten, dass ohne ein generelles Verbot offenbar nur überlebt, wer klebt: Firmen und Organisationen, die keine Werbung betreiben, haben es schwer, gegen die Omnipräsenz der großen Spieler anzukommen; das gilt selbst für wohltätige oder „wohlmeinende“ Organisationen.

Für den Einsatz von Plakaten im DaF-Unterricht lassen sich alle pädagogischen, lernpsychologischen und landeskundlichen Argumente geltend machen, die generell für den Einsatz von Bildern sprechen, ${ }^{28}$ darüber hinaus aber weitere medienspezifische, indem sie über die fast immer Aufmerksamkeit heischenden, oft darüber hinaus auch tief greifenden, interpretationsfreudigen Bilder hinaus auch Text bieten.

Die thematischen Anwendungen liegen wohl auf der Hand. Zahllose Aspekte der deutschen Geschichte von der Kaiserzeit bis heute lassen sich mit Werbe- und (ab 1916) politischen Plakaten illustrieren und dokumentieren. Das gilt für die Ereignisgeschichte, vor allem aber für oft nur schwer oder wenig unterhaltsam zu unterfütternde Themen wie Wirtschaftsentwicklung, Handel, Technik, die den Hauptgegenstand viele spezialisierter Kurse darstellen; um das Thema Werbekritik herum lassen sich politische und philosophische Diskussionen führen: Die Gedankenwelt der Frankfurter Schule und der Situationistischen Internationale etwa ist nur vor dem Hintergrund der Geistes-, und das heißt auch: Konsum- und Werbewelt der Weimarer bzw. Vierten und jungen Fünften Republik zu verstehen; Bildanalyse und -kritik sollten, wie oben zitiert, einmal ein Teil eines eigenen Unterrichtsfachs werden, man könnte also eine entsprechende Unterrichtsreihe als eine Art zivilbürgerliche Aufklärung im visuellen Zeitalter verstehen: Lehrkraft und Lernende würden gemeinsam die Sprache der Bilder lesen lernen... die Beispiele sind Legion.

Wichtig ist, zu verstehen, das Plakate auch im engeren Sinne sprachdidaktisch zum Einsatz kommen können, und zwar von der „Stunde Null“ an. Ein paar Anregungen:

28 Vgl. u.a. Bücken 1985, Lieskounig 1988, Kaminski 1990, Scherling; Schuckall 1992, Macaire; Hosch 1999, Biechele 2004. 
Man werfe ein möglichst buntes Plakat an die Wand (bzw. teile Kopien aus) und schreibe die deutschen Farbbezeichnungen in der unflektierten Form an die Tafel. Jeder Lerner mit Englischkenntnissen wird sie zuordnen können. Etwas später nutze man das gleiche Plakat bzw. Handout für die Genera und die Adjektivdeklination. Wenn es gut gewählt ist, lassen sich darüber hinaus einige Substantive erlernen und vielleicht auch Details zur Entstehungszeit klären.

Imperative werden in Lehrwerken des Öfteren mit Werbung in Verbindung gebracht; ${ }^{29}$ warum nicht authentische Werbe- und Politslogans verwenden: „Mach mal Pause“, „Pack den Tiger in den Tank“, „Wählt kommunistisch“, „Schützt Bayern" o.ä., um so zugleich etwas Landes- bzw. Regionalgeschichte zu vermitteln?

Lektionen zu Lokaladverbien und Wechselpräpositionen werden in aller Regel illustriert. Auch hier gilt: warum nicht Plakate oder andere historische Quellen nutzen, wie auch bei Vokabelübungen, etwa zu Adjektiven oder Alltagsgegenständen?

Mit Werbeplakaten lässt sich auch Umgangssprache vermitteln. So warb Mercedes-Benz vor einigen Jahren für die Taxibranche mit den Worten: „Bisschen breit? Kein Problem: Wir haben Platz“ und „Gerade hinstellen, Hand raushalten fertig ist die Haltestelle." (Art Directors Club für Deutschland 1998: keine Seitenangabe). An Wortschöpfungsbeispielen wie ,unkaputtbar“ oder „Nogger dir einen" lassen sich selbst morphologische bzw. morphosyntaktische Eigenheiten des Deutschen erklären. ${ }^{30}$

Plakate sind auch hervorragende Sprechanlässe (,was sehen Sie, für welches Produkt/welche Partei wirbt dieses Bild, aus welcher Zeit stammt das Plakat" etc.). ${ }^{31}$ Wahrscheinlich ist jedes bedeutendere sprachliche Phänomen auch über Plakate zu verdeutlichen. Am besten aber kombiniert man den reinen Sprachunterricht mit dem inhaltlichen (d.h. landeskundlich-geschichtlichen, aber auch technischen, wirtschaftlichen etc.): Warum nicht einmal Produktwerbungen aus verschiedenen Jahrzehnten in die richtige Reihenfolge bringen lassen (die Lösung ist zu begründen), Fachvokabular über Werbeplakate vermitteln und gleichzeitig, wie im Fiat-Beispiel oben, landeskundliche oder andere relevante Aspekte ins Spiel bringen? Die Möglichkeiten sind unbegrenzt. Und: Anders als früher sind heute zahllose Plakate im Internet abrufbar und daher auch sofort einsatzbereit. Notwendig für die Suche ist allerdings eine gewisse Vorbildung über Künstler, Parteien und dergleichen mehr, die zu vermitteln das bescheidene Ziel des vorliegenden Beitrags war.

\footnotetext{
${ }^{29}$ Etwa in „Tangram“ (Alke; Dallapiazza; Jan; Maenner 1998).

${ }^{30}$ Der „Witz“ bei „unkaputtbar“ ist freilich der Verstoß gegen die Regel. Ein anderes Beispiel ist „Hier werden Sie geholfen“, das man als falsches Beispiel für das unpersönliche Passiv verwenden kann.

31 Zur Arbeit mit Plakaten im DaF-Unterricht: Laveau; Nicolas; Sprenger 1988; Werbung im DaFUnterricht: Macaire; Hosch 1999: 138-148.
} 


\section{Literatur}

Adhoc-Gruppe Visuelle Kommunikation (1971): Visuelle Kommunikation Zur gesellschaftlichen Begründung eines neuen Unterrichtsfaches. In: Ehmer, Hermann K. (Hrsg.): Visuelle Kommunikation. Beiträge zur Kritik der Bewußtseinsindustrie. Köln: DuMont Schauberg, 367-373.

Adorno, Theodow W. (2003): Minima Moralia. Reflexionen aus dem beschädigten Leben. Frankfurt/Main: Suhrkamp.

Alke, Ina; Dallapiazza, Rosa-Maria; Jan, Eduard von; Maenner, Dieter (1998): Tangram. Deutsch als Fremdsprache 1A. Ismaning: Max Hueber Verlag.

Arnold, Friedrich (1977): Anschläge. Politische Plakate in Deutschland 1900-1970. Ebenhausen bei München: Langewiesche-Brandt.

Art Directors Club für Deutschland (1998): Jahrbuch 1998. Frankfurt/Main: Art-Directors-Club-Verlag.

Barthes, Roland (1957): Mythologies. Paris: Seuil.

Baudrillard, Jean (1970): La société de consommation. Paris: Éditions Denoël.

Bayrisches Hauptstaatsarchiv München (1996): Plakate als Spiegel der politischen Parteien in der Weimarer Republik. München: Ausstellungskataloge der Staatlichen Archive Bayerns Nr. 36.

Biechele, Barbara (2004): Lernen mit Bildern im Fremdsprachenunterricht: kognitiv-konstruktivistisch und interkulturell. Anmerkungen zu aktuellen Paradigmen. In: Meiji University International Exchange Programs Guest Lecture Series 3, $1-38$.

Bloch, Ernst (1985): Das Prinzip Hoffnung. Frankfurt/Main: Suhrkamp.

Bösch, Frank (2008): „Keine Experimente“. Adenauer als alternder Staatsmann. In: Paul, Gerhard (Hrsg.) (2008): Das Jahrhundert der Bilder. Band 2: 1949 bis heute. Göttingen: Vandenhoeck \& Ruprecht, 194-201.

Brommer, Peter; Krümmel, Achim; Werner, Wolfram (2002): Geschichte plakativ. Das 20. Jahrbundert am Mittelrhein in Plakaten und Flugblättern. Koblenz: Görres.

Bücken, Hajo (Hrsg.) (1985): Bilder und was man damit machen kann. Offenbach: Burckhardthaus-Laetare.

Brune, François (1985): Le bonheur conforme. Essai sur la normalisation publicitaire. Paris: Gallimard.

Brune, François (1996): „Les médias pensent comme moi!“ Fragments du discours anonyme. Paris/Montréal: L'Harmattan. 
Brune, François, (2004): De l'idéologie, aujourd'bui. Analyses, parfois désobligeantes, du „,discours" médiatico-publicitaire. Paris: Parangon.

Buchli, Hanns (1962): 6000 Jabre Werbung. Geschichte der Wirtschaftswerbung und der Propaganda. Band 1: Altertum und Mittelalter. Berlin: de Gruyter.

Buchli, Hanns (1966): 6000 Jahre Werbung. Geschichte der Wirtschaftswerbung und der Propaganda. Band 3: Das Zeitalter der Revolutionen. Berlin: de Gruyter.

Debord, Guy (1970): La société du spectacle. Paris: Éditions Buchet-Chastel.

Debord, Guy (1988): Commentaires sur la société du spectacle. Paris: Gallimard.

Döring, Jürgen (2008): Die blutige Uniform. Oliviero Toscani und die „BenettonPlakate“. In: Paul, Gerhard (Hrsg.) (2008), 629-637.

Eicke, Ulrich (1991): Die Werbelawine. Angriff auf unser Bewußtsein. München: Knesebeck \& Schuler.

Fahlenbrach, Katrin (2008): Die Grünen. Neue Farbenlehre der Politik. In: Paul, Gerhard (Hrsg.) (2008), 474-481.

Garcin, Jérôme (Hrsg.) (2007): Nouvelles mythologies. Paris: Seuil.

Gries, Rainer; Ilgen, Volker; Schindelbeck, Dirk (1995a): Einleitung. Kursorische Überlegungen zu einer Werbegeschichte als Mentalitätsgeschichte. In: Dies. (Hrsg.) (1995), „Ins Gehirn der Masse kriechen!“Werbung und Mentalitätsgeschichte. Darmstadt: Wissenschaftliche Buchgesellschaft, S. 1-28.

Gries, Rainer; Ilgen, Volker; Schindelbeck, Dirk (1995b): Magische Formeln. „Mach mal Pause“ ? „Keine Experimente!“. Zeitgeschichte im Werbeslogan. In: Dies. (Hrsg.) (1995), 92-105.

Grohnert, René (2007): „Unsere Epoche ist die Plakatischste!“? Das deutsche Plakat vor 1914. In: Ders. (Hrsg.) (2007): Ein Leben nach den Kleben. ZEITZEIGER. Plakate aus zwei Jahrhunderten. Essen/Mainz: Verlag Hermann Schmidt, 76-79.

Hagen, Manfred (21984): Werbung und Angriff - Politische Plakate im Wandel von hundert Jahren. In: Bohrmann, Hans (Hrsg.): Politische Plakate. Dortmund: Harenberg, 49-70.

Hartwig, Helmut (1971): Visuelle Kommunikation. Methodologische Bemerkungen zur Ableitung von Lernzielen aus dem sozialen Bereich „Freie Zeit“. In: Ehmer, Hermann K. (Hrsg.) (1971), 334-339.

Haubl, Rolf (1992): „Früher oder später kriegen wir euch“. In: Hartmann, Hans A.; Haubl, Rolf (Hrsg.): Bilderflut und Sprachmagie. Fallstudien zur Kultur der Werbung. Opladen: Westdeutscher Verlag, 9-32. 
Huber, Kurt (1990): Image. Global-Image, Corporate-Image, Marken-Image, Produkt-Image. Landsberg/Lech: Verlag Moderne Industrie.

Huxley, Aldous (1958): Brave New World Revisited. New York: Harper \& Row.

Ingenkamp, Konstantin (1996): Werbung Gesellschaft. Hintergründe und Kritik der Kulturwissenschaftlichen Reflexion von Werbung. Frankfurt/Main: Peter Lang.

Kaminski, Diethelm (1990): Lernideen mit Bildern. Ismaning: Verlag für Deutsch.

Kühnel, Anita (2007): Zwischen Innovation und Professionalisierung. Plakate der 1920er und -30er Jahre. In: Grohnert, René (Hrsg.) (2007), 142-145.

Lamberty, Christiane (2000): Reklame in Deutscbland 1890-1914. Wabrnehmung, Professionalisierung und Kritike der Wirtschaftswerbung. Berlin: Duncker \& Humboldt.

Laveau, Inge; Nicolas, Gerd; Sprenger, Margret (1988): Bild als Sprechanlaß. Werbeanzeigen. München: Goethe Institut.

Les Désobéissants (2009): Désobéir à la Pub. Le Pré-Saint-Gervais: Le passager clandestin.

Macaire, Dominique; Hosch, Wolfram (1999): Bilder in der Landeskunde. Berlin u.a.: Langenscheidt.

Lieskounig, Juergen (1988): Durch Bilder zur Sprache? - Überlegungen zur Problematik visueller Mittel in Lehrwerken Deutsch als Fremdsprache. In: Zielsprache Deutsch 9, 2-8.

Malhotra, Ruth (1984): Künstler und politisches Plakat. In: Bohrmann, Hans (Hrsg.) (1984), 11-48.

Marcuse, Herbert (1967): Der eindimensionale Mensch. Studien zur Ideologie der fortgeschrittenen Industriegesellschaft. Neuwied und Berlin: Luchterhand.

Medebach, Friedrich (1941): Das Kamppplakat. Aufgabe, Wesen und Gesetzmäßigkeit des politischen Plakats, nachgewiesen an den Plakaten der Kampfjahre von 1918-1933. Limburg: Limburger Vereinsdruckerei.

Messaris, Paul (1997): Visual Persuasion. The Role of Images in Advertising. London: Sage.

Miller, Geoffrey (2010): Must-Have. The Hidden Instincts Bebind Everything We Buy. London: Vintage Books.

Möller, Heino R. (1971): Kunstunterricht und Visuelle Kommunikation. Sieben Arbeitsthesen zur Konzeption eines neuen Unterrichtsfaches. In: Ehmer, Hermann K. (Hrsg.) (1971), 363-366.

Oeil-de-Boeuf, Josée (2010): Marketing, disent-ils. Dix-buit analyses navrées de la publicité contemporaine. Paris: Le Tigre. 
Offensive (Hrsg.) (2010): Divertir pour dominer. La culture de masse contre les peuples. Montreuil: Éditions l'Échappée.

Packard, Vance (1962): Die geheimen Verführer. Der Griff nach dem Unbewußten in jedermann. Berlin: Ullstein.

Paul, Gerhard (2008): „Alle Wege des Marxismus führen nach Moskau“. Schlagbilder antikommunistischer Bildrhetorik. In: Ders. (Hrsg.) (2008), 88-97.

Ramonet, Ignacio (2000): Propagandes silencieuses. Masses, télévision, cinéma. Paris: Gallimard.

Rebel, Ernst (22009): Druckgrafik. Geschichte und Fachbegriffe. Stuttgart: Reclam.

Rebel, Ernst (2010): Meisterwerke der Druckgrafik. Stuttgart: Reclam.

Rücker, Matthias (2000): Wirtschaftswerbung unter dem Nationalsozialismus. Rechtliche Ausgestaltung der Werbung und Tätigkeit des Werberats der deutschen Wirtschaft. Frankfurt/Main: Peter Lang.

Scherling, Theo; Schuckall, Hans Friedrich (1992): Mit Bildern lernen. Handbuch Fremdsprachenunterricht. Berlin u.a.: Langenscheidt.

Schierl, Thomas (2005): Werbungsforschung. In: Sachs-Hombach, Klaus (Hrsg.) (2005): Bildwissenschaft. Disziplinen, Themen, Methoden. Frankfurt/Main: Suhrkamp, 309-319.

Schindelbeck, Dirk (1992): Hans Domizlaff oder die Ästhetik der Macht. Eines Werbeberaters Geschichte. In: Geschichtswerkstatt 25: Werbung als Geschichte, 13-30.

Schönert, Walter (1988): Werbung, die ankommt. 199 Beispiele, Erfolgsregeln, praktische Folgerungen. Düsseldorf: Econ.

Seidensticker, Mike (1995): Werbung mit Geschichte. Ästhetik und Rhetorik des Historischen. Köln u.a.: Böhlau.

Staeck, Klaus (2008): Wie alles anfing. In: Ders. (2008): Plakate. Göttingen: Steidl, 109-136.

Vorsteher, Dieter (2007): Bilder für den Sieg. Das Plakat im Ersten Weltkrieg. In: Grohnert (Hrsg.) (2007), 123-125.

Westphal, Uwe (1989): Werbung im Dritten Reich. Berlin: Transit. 



\section{Autoren}

Jens Grimstein, M.A.

Université Paris Est Créteil (UPEC)

UFR Lettres et Sciences Humaines

Département d'allemand

F-94010 Créteil Cedex

jens.grimstein@u-pec.fr

\section{Dr. Marc Hieronimus}

Université de Picardie Jules Verne

Faculté de Langues

Section d'Allemand

F-80025 Amiens

marc.hieronimus@u-picardie.fr

Wolfgang Koller, M.A.

Lutherstr. 7

D-34117 Kassel

wolfgangkoller@yahoo.de

\section{Prof. Dr. Uwe Koreik}

Universität Bielefeld

Fakultät für Linguistik und Literaturwissenschaft

D-33501 Bielefeld

uwe.koreik@uni-bielefeld.de

\section{Dr. Arndt Kremer}

University of Malta

Department of German

MSD-2080 Malta

kremer.arndt@web.de 
PD Dr. Jürgen Nielsen-Sikora

Universität zu Köln

Historisches Institut

Abteilung Didaktik der Geschichte und

Geschichte der Europäischen Integration

D-50931 Köln

juergen.sikora@uni-koeln.de

\section{Dr. Thomas Roth}

NS-Dokumentationszentrum

EL-DE-Haus

Appellhofplatz 23-25

D-50667 Köln

thomas.roth@stadt-koeln.de

Dr. Joachim Sistig

Ruhr-Universität-Bochum

Romanisches Seminar

GB 8/140

D-44780 Bochum

sjsistig@aol.com

\section{Beatrice Wiegand, M.A.}

Doktorandin am Seminar für Sprechwissenschaft und Phonetik

Martin-Luther-Universität Halle-Wittenberg

D-06114 Halle 
Die Beiträge des vorliegenden Bandes gehen auf eine Fachtagung des Deutschen Akademischen Austauschdienstes (DAAD) im März 2011 zurück. Ihr lag eine simple Überlegung zugrunde: Im Sprach- und Landeskundeunterricht sind Medien unerlässlich - warum nicht auf historische Quellen zurückgreifen? Sie sind vielseitig einsetzbar, haben einen hohen Wiedererkennungswert und bieten unzählige Anknüpfungspunkte an landeskundliche und medienwissenschaftliche Themen. Die Beiträge einschlägiger Fachwissenschaftler stellen zunächst die wichtigsten Sammlungen und Portale im Internet vor und fragen nach Aussagekraft und Praxiswert historischer Quellen. Eingehender behandelt werden sodann die Quellen Tondokument, Spielfilm, Karikatur und Plakat sowie Straßennamen und Texte der Arbeiterliteratur.

\section{fadaf $f$}

fadaf.de

ISBN: 978-3-86395-061-3

ISSN: 1866-8283 